\title{
ULTRA LOW DENSITY SHAPE MEMORY POLYMER FOAMS WITH TUNABLE PHYSICOCHEMICAL PROPERTIES FOR TREATMENT OF INTRACRANIAL ANEURYSMS
}

\author{
A Dissertation \\ by \\ POOJA SINGHAL
}

\begin{abstract}
Submitted to the Office of Graduate Studies of Texas A\&M University in partial fulfillment of the requirements for the degree of DOCTOR OF PHILOSOPHY
\end{abstract}

$\begin{array}{ll}\text { Chair of Committee, } & \begin{array}{l}\text { Duncan J. Maitland } \\ \text { Committee Members, } \\ \text { Elizabeth Cosgriff-Hernandez } \\ \text { Jaime Grunlan } \\ \text { Melissa Grunlan } \\ \text { Thomas S. Wilson }\end{array} \\ \text { Head of Department, } & \text { Gerard Cote }\end{array}$

August 2013

Major Subject: Biomedical Engineering

Copyright 2013 Pooja Singhal 


\begin{abstract}
Shape memory polymers (SMPs) are a rapidly emerging class of smart materials that can be stored in a deformed temporary shape, and can actively return to their original shape upon application of an external stimulus such as heat, $\mathrm{pH}$ or light. This behavior is particularly advantageous for minimally invasive biomedical applications comprising embolic and regenerative scaffolds, as it enables a transcatheter delivery of the device to the target site. The focus of this work was to exploit this shape memory behavior of polyurethanes, and develop an efficient embolic SMP foam device for the treatment of intracranial aneurysms.

The first step herein was to achieve ultra low density foams with a highly covalently crosslinked polymer structure to enable good mechanical properties and shape memory behavior, while ensuring a high filling efficiency of the device. Favorable results of densities down to $0.015 \mathrm{~g} \cdot \mathrm{cm}^{-3}$ were attained with excellent shape memory of $98-99 \%$ recovery and up to 70 times volume expansion on actuation for these materials. However, these materials showed premature actuation on exposure to water/physiological media during delivery through a microcatheter. This hindered the device delivery, and inspired a new series of materials with controlled hydrophobicity. Successful transcatheter delivery has since been conducted with these materials. Additional optimization of the foam formulation and synthesis process produced still lower densities of down to $0.008 \mathrm{~g} \cdot \mathrm{cm}^{-3}$ providing a further improved embolic efficiency. Also, plasma treatment of the foams was identified as a viable and efficient
\end{abstract}


technique for achieving complete reticulation of these materials, important for moderating the permeability of the device. Finally, low molecular weight ester based monomers were introduced in the foams to achieve controlled device degradation and avoid long presence of a foreign material in the body.

In summary, this work reports a novel family of ultra low density polymer foams which can be delivered via a minimally invasive surgery to the aneurysm site, actuated in a controlled manner to efficiently embolize the aneurysm while promoting physiological fluid/blood flow through the reticulated/open porous structure, and eventually biodegrade leading to complete healing of the vasculature. 
To the Almighty God who showed me the way,

To my dear husband, Nikhil, who held me close whenever I would sway, and, To my family for their endless love and support night and day... 


\section{ACKNOWLEDGEMENTS}

Foremost, I would like to express my gratitude to my co-advisors Dr. Duncan J. Maitland and Dr. Thomas S. Wilson for providing me an incredible graduate education. I will fondly recall the last 5 years as a remarkable journey through a wild maze of sometimes traversable and sometimes seemingly insurmountable challenges. Had it not been for their unfettered support and guidance throughout, I would not have found the way; or if I did, it could possibly not have been as rewarding and enriching.

I am extremely thankful to Dr. Maitland for mentoring me, and giving the perfect balance of academic and industrial flavor to my graduate experience. Working on a medical device, and seeing it advance through FDA regulations, almost simultaneously, has been truly exhilarating for me. Along the way, Dr. Maitland's translational approach, and continual innovation towards achieving simple real-world solutions to complicated problems have stimulated me to think in new dimensions, and helped me mature as an engineering professional. Ph.D. is as much a personal journey, as it is technical; Dr. Maitland's excellent people-skills, and his open and friendly bond with his students have inspired tremendous personal growth in me. It has been heartening to see his constant endeavor to, time and again, step in our shoes, recall his own life's experiences and try to find the most equitable, unbiased and generous ways, to deal with us. He has always put his students first, and I have come to respect him deeply for this ability and desire of his.

I am very thankful to Dr. Wilson for his time and patience during my graduate 
work. His mentorship has helped me evolve in numerous ways, personally and professionally. Coming from a strong industrial background, he has challenged me at all levels - from safe handling of chemicals in the lab and attention to detail, to brainstorming new technical advances - and enabled a rational and systematic thought process in everything I do. His expertise in material science has enhanced my ability to apply the material structure-property relationships in practical and industrially relevant terms. His scientific writing skills, and diligence with flow and continuity of thought in writing, have developed my ability to review my papers critically and produce quality technical documents. I am thankful for the opportunity to learn and grow in his guidance. I could not have asked for a more elevating graduate experience.

I would also like to thank my other committee members, Drs. Elizabeth CosgriffHernandez, Jamie Grunlan, and Melissa Grunlan. I have been extremely fortunate to have a committee that has been so supportive of me. I would like to thank Dr. CosgriffHernandez for her guidance throughout, especially in the development of biodegradable materials. Her technical insight and thought provoking questions have helped me gain a higher level of maturity in my ways of presenting technical data, and planning well thought-out experimental studies. Thanks to Dr. Melissa Grunlan and Dr. Jamie Grunlan for their guidance and support in all the major steps that I took during my graduate education. Their unremitting approval of my work and abiding belief in me has been very encouraging, and has perpetually motivated me to outperform expectations.

I am grateful for the financial support for my graduate education, provided by NIH, and particularly by the LLNL's Lawrence Scholar Program. Working at LLNL 
during my graduate education has provided me a unique experience and helped me grow by leaps and bounds professionally. I want to thank Christine, Mary, Jackie and Helen for assisting me with critical administrative tasks at LLNL, and taking the time to thoroughly research each of my "foreign national" questions. At A\&M, I am grateful to the support and assistance provided by Barbara, Andy, Chanille, Nicole, Maria, Fidel, Barry and Kayla. Thanks to Barbara for her continuous help with orders and reservations; and for always being there with comforting words and a caring smile. Thanks to Andy for co-operation in multiple undertakings, while setting up the new lab, with a cheerful attitude that always made things look much easier. Thanks to Chanille, Nicole, Maria and Fidel for their assistance in ensuring my smooth transition from A\&M to LLNL, and helping me with the registration every single semester. Thanks to Barry and Kayla for aptly handling the financial aspects. I could always count on them for prompt response to questions and for making sure everything happened as planned.

My graduate research experiences would not have been the same without Dr. Ward Small. I can never thank Ward enough for his relentless support and guidance throughout. He has been a wonderful calming presence at work with whom I could discuss my personal thoughts as well as technical ideas. When in need, I could always count on him for encouragement and caring words. His keen observation and excellent writing skills have always been an inspiration, and a tremendous support to me in technical writing. Thanks to Dr. Steve Letts for his expert guidance in FTIR analysis. I am so glad that I got a chance to work with him just in time before he left the lab. I would probably never have come to love FTIR so much if it was not for him. Thanks to 
Dr. Michael Stadermann and Dr. Suhas Bhandarkar for enabling me to work in the B162 lab, and for going out of their way to iron out the technical and administrative hurdles in allowing a foreign national to use a NIF affiliated lab. I would like to thank Marilyn Brooks for being an awesome undergraduate intern. She was a wonderful company and a great asset at the opportune time when voluminous amounts of foams had to be cranked out. Thank you, Matt Davenport, for being such a co-operative officemate with whom I could share multiple stories and the wows and woes of graduate school. At A\&M, thanks to Tony, Jennifer, Wonjun, Ya-Jen, Keith and Andrea for providing numerous sweet memories for life. I would also like to thank Matt, Richard, and Casey; it has been a privilege working with and mentoring you all.

Last, but not the least, I am extremely thankful to my family. To Mom and Dad: Thank you for supporting me, loving me, leading me, teaching me, inspiring me, believing in me, sometimes tolerating me, and for always helping me follow my dreams throughout my life. Thank you for instilling in me your great ideals for life; without them, I would have been long lost. To brother and sister: Thank you for being the most awesome companions and biggest inspirations for me through all the times. Your loving and encouraging words have always helped me move past all hurdles. To Mom and Dad in-law: Thank you for your endless and unconditional love for me ever since I have known you. The sentiment of affection and joy, that pervades your life and whole existence, is an inspiration for me to live and follow. And most importantly, to Nikhil: Thank you for your boundless love and support through all my endeavors. This dissertation would not have been possible without you. 


\section{NOMENCLATURE}

\begin{tabular}{|c|c|}
\hline$\Delta T$ & Range of transition $\left[=2 \cdot\left(T_{\delta s}-T_{\text {onset }}\right)\right]$ \\
\hline$\Delta_{T}$ & Lyderson correction for non-ideality \\
\hline$\Delta_{T, i}$ & Lyderson correction for non-ideality for the $i^{\text {th }}$ component \\
\hline$\Delta_{T}^{(P)}$ & Non-ideality correction factor for polymers \\
\hline \multirow[t]{2}{*}{ ATR-FTIR } & Attenuated total reflectance - fourier transform infra-red \\
\hline & spectroscopy \\
\hline $\mathrm{CFC}$ & Chlorofluorocarbon \\
\hline CLS & Critical limiting supersaturation \\
\hline DI & De-ionized \\
\hline DMTA & Dynamic mechanical thermal analysis \\
\hline $\mathrm{DSC}$ & Differential scanning calorimeter \\
\hline ELISA & Enzyme linked immuno sorbent assay \\
\hline$E_{\text {neat }}$ & Young's modulus of neat/non-porous material \\
\hline$E_{\text {porous }}$ & Young's modulus of porous material \\
\hline FDA & U.S. food and drug administration \\
\hline$F_{p}$ & Polar component of the molar attraction function \\
\hline \multirow[t]{2}{*}{$F_{p, i}$} & Polar component of the molar attraction function for the $i^{\text {th }}$ \\
\hline & component \\
\hline$F_{t}$ & Molar attraction function \\
\hline$F_{t, i}$ & Molar attraction function for the $i^{\text {th }}$ component \\
\hline
\end{tabular}


FTIR

$G^{\prime}$

$G^{\prime \prime}$

GDC

$G_{\text {neat }}^{\prime}$

$G_{\text {porous }}^{\prime}$

HCFC

$\mathrm{HCl}$

HDI

HFC

HIPE

HPED

IPDI

IR

ISAT

LPS

LLNL

$\mathrm{NaOH}$

$n$

$n_{c}$

$N_{i}$
Fourier transform infrared spectroscopy

Storage modulus

Loss modulus

Guglielmi detachable coil

Storage modulus of neat/non-porous material

Storage modulus of porous material

Hydrochlorofluorocarbon

Hydrochloric acid

1, 6 Diisocyanatohexane

Hydrofluorocarbon

High internal phase emulsion

$\mathrm{N}, \mathrm{N}, \mathrm{N}^{\prime}, \mathrm{N}^{\prime}$ Tetrakis (2-hydroxypropyl) ethylenediamine

5-Isocyanato-1-(isocyanatomethyl)-1,3,3-trimethyl cyclohexane;

Isophorone diisocyanate

Infrared

International subarachnoid aneurysm trial

Liposaccharide

Lawrence livermore national laboratory

Sodium hydroxide

Number of repeating units per effective polymer chain segment

Crosslink density (number of active chains per unit volume)

Number of units of $i^{\text {th }}$ component per effective polymer chain 
segment

NIF

National ignition facility

$\mathrm{NIH}$

National institute of health

$N_{h}$

Degree of polymerization of the hard segment

$\mathrm{OH} / \mathrm{NCO}$ ratio

Ratio of the hydroxyl to isocyanate equivalents in a composition

PCL

Polycaprolactone; $(1,7)$ polyoxepan-2-one

PCL-t

Polycaprolactone triol

PFC

Perfluorocarbon

PHA

Phytohemagglutinin

$R$

Ideal gas constant $\left(8.314 \mathrm{~J} \cdot \mathrm{K}^{-1} \cdot \mathrm{mol}^{-1}\right)$

SMA

Shape memory alloy

$\mathrm{SMC}$

Shape memory ceramic

SMP

Shape memory polymer

$T$

$\tan \delta$

Temperature

TEA

2,2',2" Nitrilotriethanol

$T_{g}$

$T_{\text {high }}$

$T_{\text {low }}$

Glass transition temperature

A temperature above the transition temperature $\left(\sim T_{\text {trans }}+30^{\circ} \mathrm{C}\right)$

A temperature below the transition temperature $\left(\sim T_{\text {trans }}-30{ }^{\circ} \mathrm{C}\right)$

TMHDI

2,4,4 (and 2,2,4) Trimethyl 1,6 diisocyanatohexane

$T_{\text {onset }}$

Temperature at which glass transition onset is observed

$T_{\text {trans }}$

Shape memory transition temperature; actuation temperature 


\begin{tabular}{|c|c|}
\hline$T_{\delta s}$ & Temperature at which peak value of $\tan \delta$ is achieved \\
\hline$V$ & Molar volume \\
\hline$V_{i}$ & Molar volume of the $i^{\text {th }}$ component \\
\hline$\alpha_{(P)}$ & Molecular aggregation number for amorphous polymers \\
\hline$\delta_{d}$ & Solubility parameter contribution of dispersive forces \\
\hline$\delta_{h}$ & Solubility parameter contribution of hydrogen bonding \\
\hline$\delta_{p}$ & Solubility parameter contribution of polar forces \\
\hline$\delta_{t}$ & Solubility parameter (total) \\
\hline$\varepsilon_{\text {pre }}$ & Initial strain \\
\hline$\varepsilon_{\text {rec }}$ & Recovered strain \\
\hline$\rho_{\text {neat }}$ & Density of neat/non-porous material \\
\hline$\rho_{\text {porous }}$ & Density of porous material \\
\hline$\sigma_{e l}$ & Stress in the elastic regime \\
\hline$\sigma_{p l}$ & Stress in the plastic regime \\
\hline$\chi$ & Flory - Huggins interaction parameter \\
\hline
\end{tabular}




\section{TABLE OF CONTENTS}

Page

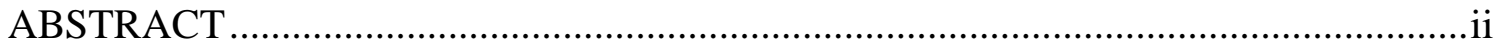

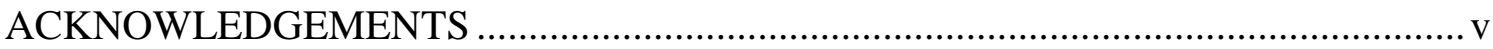

NOMENCLATURE..........................................................................................

TABLE OF CONTENTS .......................................................................................ii

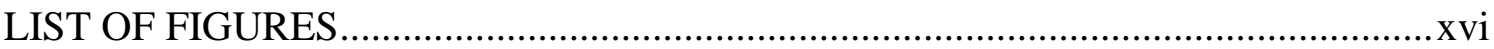

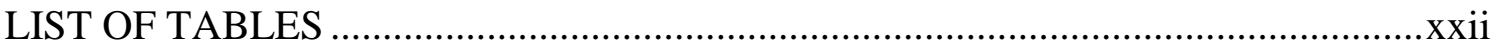

CHAPTER I INTRODUCTION AND LITERATURE REVIEW ................................ 1

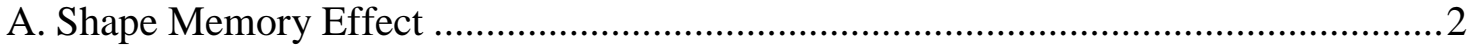

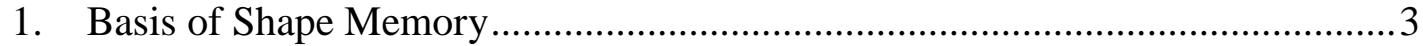

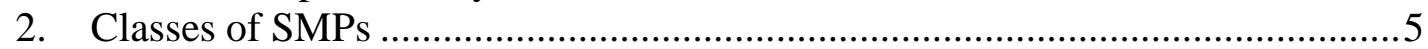

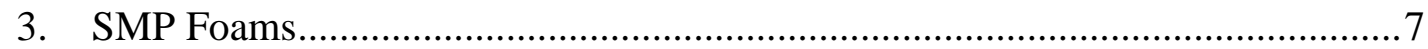

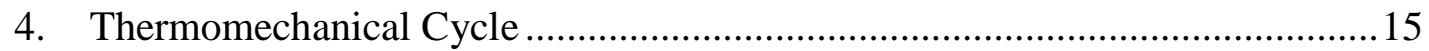

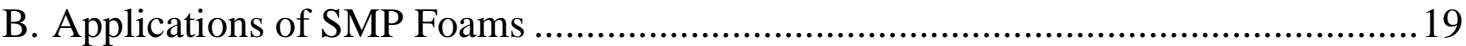

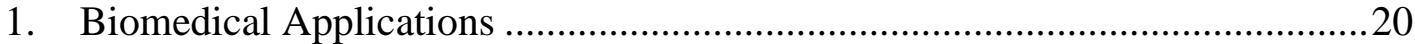

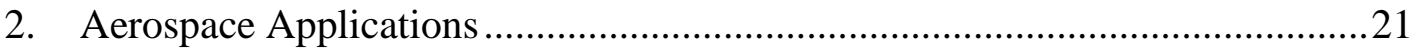

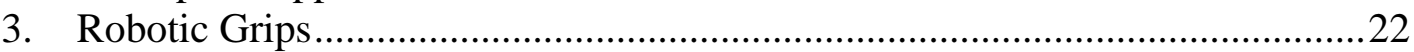

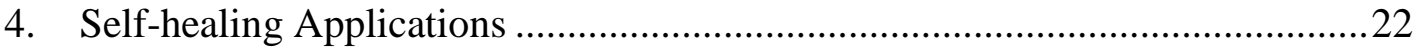

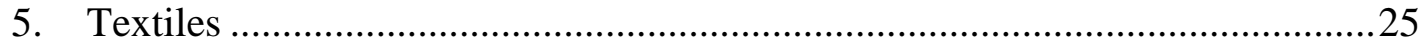

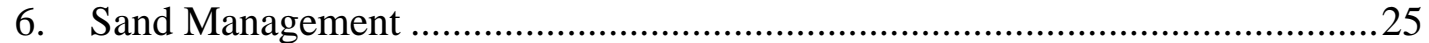

C. Use of SMP Foams for Treatment of Intracranial Aneurysms ……………….......26

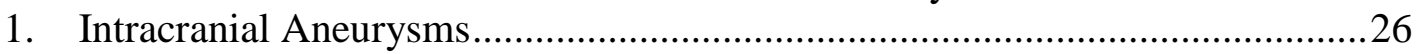

2. Current Treatments of Intracranial Aneurysms .............................................28

3. Shape Memory Polymer Foams for Aneurysm Treatment...................................32

4. Limitations of Currently Available SMP Foams for this Application.................33

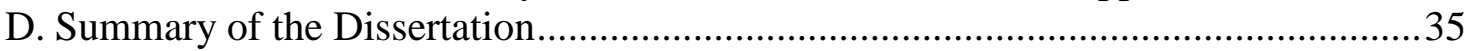

\section{CHAPTER II ULTRA LOW DENSITY AND HIGHLY COVALENTLY}

CROSSLINKED SHAPE MEMORY POLYURETHANE FOAMS ...............................37

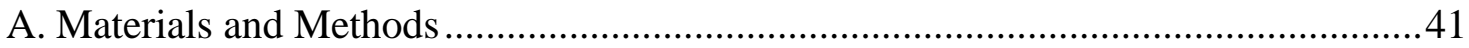




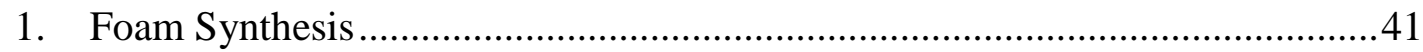

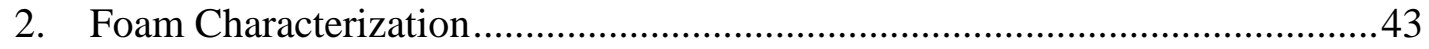

B. Results and Discussion....................................................................................

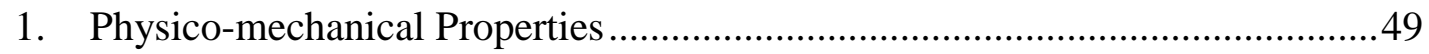

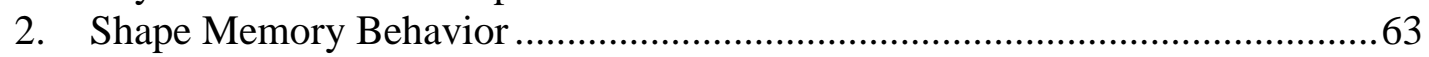

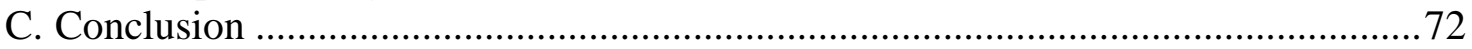

\section{CHAPTER III CONTROLLING THE ACTUATION RATE OF SHAPE MEMORY}

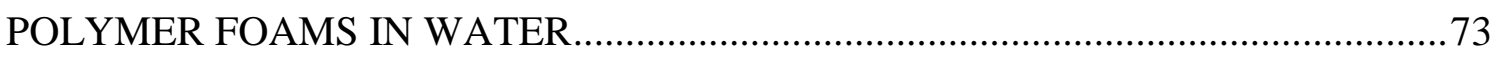

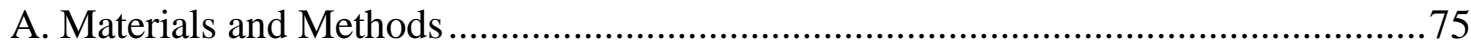

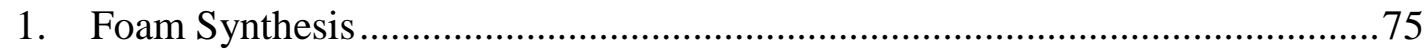

2. Post-processing of Foams ………………………........................................

3. Characterization of Physical Properties............................................................ 79

4. Characterization of Foam Actuation and Actuation-related Properties..............81

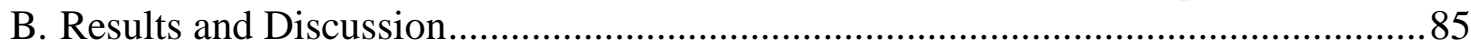

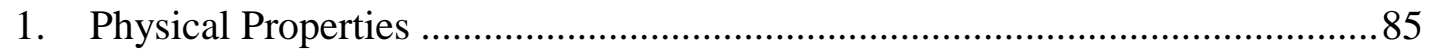

2. Characterization of Foam Actuation and Actuation-related Properties..............92

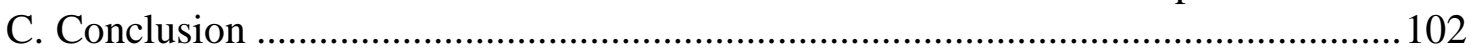

\section{CHAPTER IV SYNTHESIS AND POST-SYNTHESIS MODIFICATIONS FOR}

REDUCED DENSITIES AND COMPLETE RETICULATION OF FOAMS ..............103

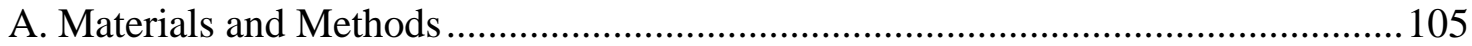

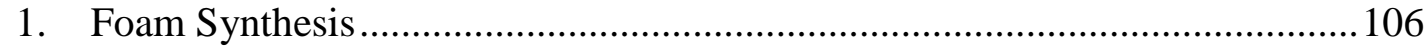

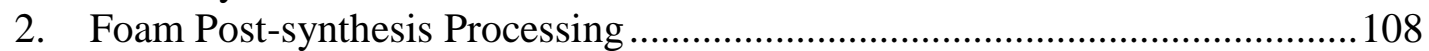

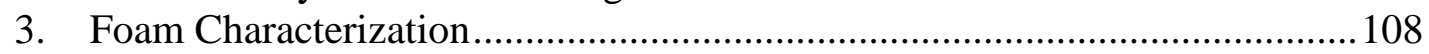

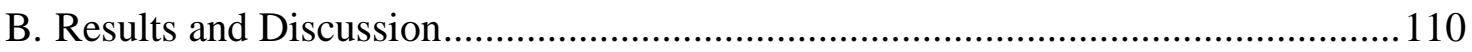

1. Proposed Mechanism of Process Modifications ...............................................110

2. Proposed Mechanism of Post- processing by Plasma Treatment ......................114

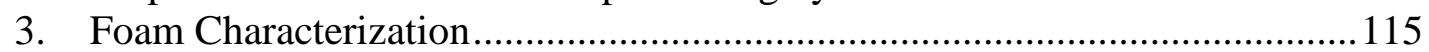

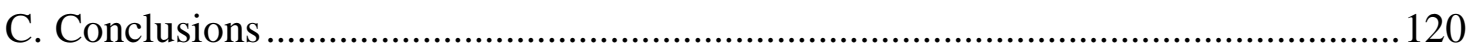

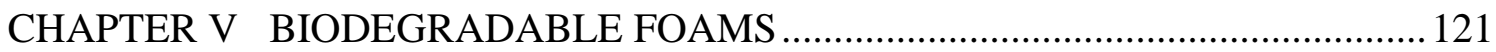

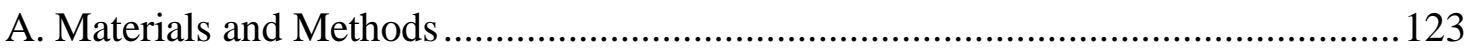

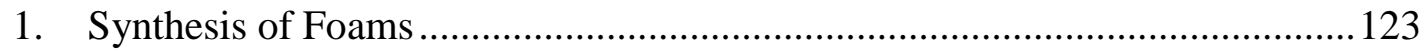

2. Characterization of Physical Properties........................................................125

3. Characterization of Degradation Behavior ………….....................................127

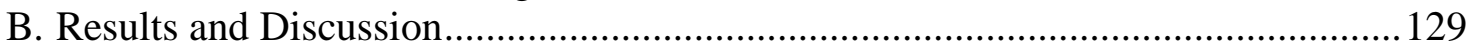

1. Physical Properties Characterization .............................................................129

2. Characterization of Degradation Behavior ........................................................137 


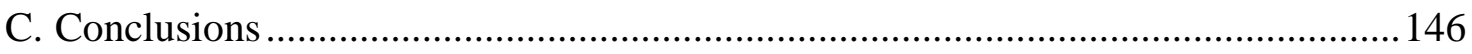

CHAPTER VI CONCLUSIONS AND FUTURE WORK ........................................... 148

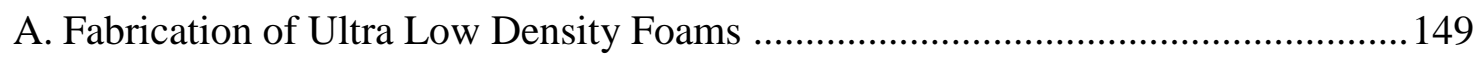

B. Controlled Variation in the Material Hydrophilicity ...............................................151

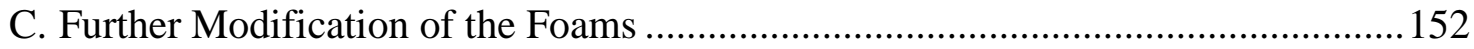

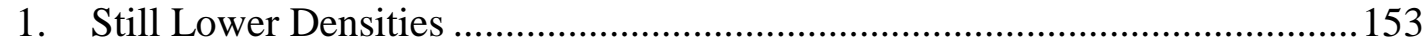

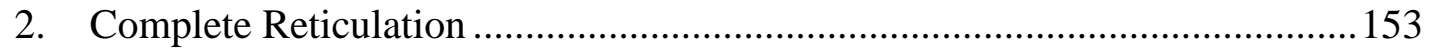

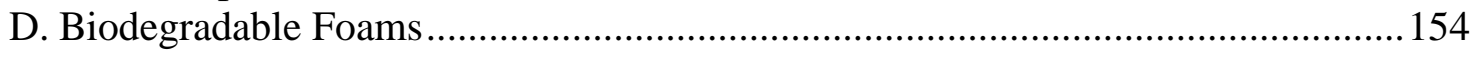

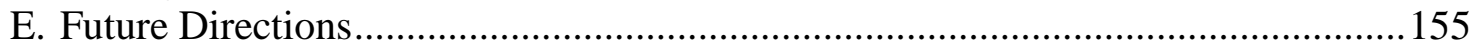

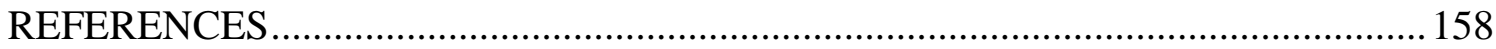

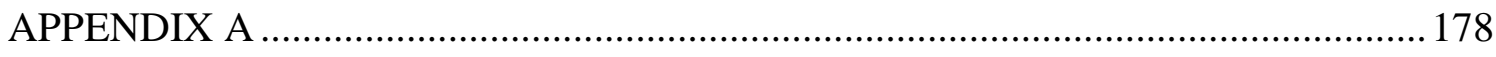

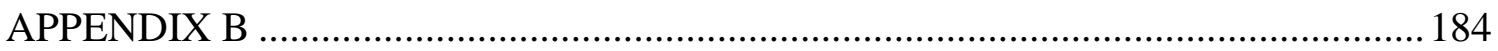

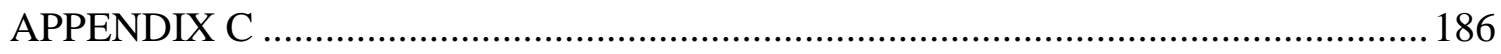

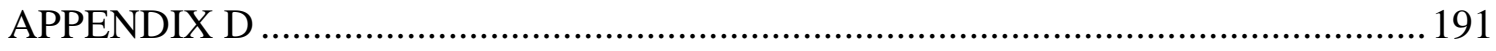




\section{LIST OF FIGURES}

Page

Figure 1: The volume of SMP research has seen a near exponential rise in the recent decades. This figure is adapted with permission from [1].

Figure 2: Schematic of the thermomechanical cycle for shape memory polymers in compression mode. Large compressive strain is imposed along A (Loading), the SMP is cooled along B to lock in the temporary compressed shape (Fixing), the material is then allowed to recover either by free strain recovery along $\mathrm{C}_{\mathrm{i}}$, or by constrained stress recovery along $\mathrm{C}_{\mathrm{ii}}$. A final step of unloading along $\mathrm{D}$ is performed after the constrained stress recovery $\mathrm{C}_{\mathrm{ii}}$.

Figure 3: Schematic representation of changes in the polymer chain orientation, and the physical shape of a SMP foam as it is taken through the thermomechanical cycle. Image courtesy: Dr. Thomas S. Wilson [demonstration of the programming and physical shape transformations of a SMP foam device in the bottom panel].

Figure 4: Schematic of the self-healing scheme of the proposed smart foams $\left(T_{g}\right.$ is the transition temperature of the foams). The figure is reproduced with permission from [31].

Figure 5: C-scan images of the sandwich panels after each impact and healing cycle. The figure is reproduced with permission from [47]. C-scan images give a visual representation of the attenuation of the ultrasound signal through the panel, which is proportional to the damage present, with $20 \%$ attenuation indicated by red color, $20-50 \%$ attenuation by blue color and $100 \%$ attenuation by white color. As the figure shows, even after the $7^{\text {th }}$ impact, the macro-length scale damage can be adequately healed by heating the foam above its $T_{g}$.

Figure 6: Rupture of an intracranial aneurysm may cause hemorrhage, and it is often fatal to the patient.

Figure 7: Surgical clipping: A technique for treatment of aneurysms where a metal clip is surgically placed at the aneurysm neck to avoid blood flow into it.......29

Figure 8: Treatment of aneurysm using Guglielmi detachable coils. 
Figure 9: Schematic showing treatment of aneurysms, progressing from top left depicting an untreated aneurysm, to bottom right depicting an implanted foam device (Image Courtesy: Jennifer N. Rodriguez).

Figure 10: Molecular structures of the low molecular weight, symmetrical and polyfunctional monomers (HPED, TEA and HDI) used in the SMP foam synthesis.

Figure 11: A schematic representation of the network structure of (a) physically crosslinked traditional polyurethane foams and (b) proposed covalently crosslinked foams from low molecular weight monomers. This figure is reproduced with permission from [30].

Figure 12: Representative cell structure of foams for all the formulations H80 through H0. Foams of all formulations show a mixed, closed to open cell structure, with thin cell membranes between struts. The residual thin cell membranes can be seen in the magnified image. All scale bars are $500 \mu$ except the magnified image where the scale bar is $200 \mu$. This figure is reproduced with permission from [30].

Figure 13: Histogram showing cell size distribution of H60 foam. It is seen that the pore cells are anisotropic, being taller, approximately $1000 \mu$, than they are wide, approximately $700 \mu$. Here equatorial measurements represent lateral diameters of pores, perpendicular to the direction of foaming; and the polar measurements represent the longitudinal diameters of pores parallel to the direction of foaming. This figure is reproduced with permission from [30].

Figure 14: A comparison between the FTIR-ATR spectra of an unfoamed neat polymer (offset by 0.03 absorbance units) and foam of corresponding formulation. This figure is reproduced with permission from [30].

Figure 15: Some important features are observed in the FTIR spectra: 1) presence of a shoulder in the urethane carbonyl bond (1620-1660 $\left.\mathrm{cm}^{-1}\right)$ indicating presence of urea from chemical foaming with water (b); 2) presence of hydrogen bonding due to urea groups also shows a broadening of the $\mathrm{N}$ $\mathrm{H}$ peak (3100-3500 $\mathrm{cm}^{-1}$ ) (c); and 3) a highly compact and hydrogen bonded structure is estimated as the urethane bond shows a shift to lower wavenumber $\left(1689 \mathrm{~cm}^{-1}\right)$ compared to traditional foams (b). This figure is reproduced with permission from [30].

Figure 16: DSC curves for each foam formulation. A variation in $T_{g}$ of approximately $45-70{ }^{\circ} \mathrm{C}$ was achieved by varying the ratio of HPED to TEA in the foam formulation. A higher HPED content gave a higher glass transition value. This is likely due to the increase in rotational 
steric hindrance due to secondary hydroxyl, and increase in the crosslink density due to the use of tetrafunctional HPED. This figure is reproduced with permission from [30].

Figure 17: DMTA curves of H0-H80 samples. Variation in transition temperatures is in agreement with the DSC results. This figure is reproduced with permission from [30].

Figure 18: Shape memory cycles performed on a $\mathrm{H} 20$ foam sample $\left(T_{g} \sim 50{ }^{\circ} \mathrm{C}\right)$ in a constrained stress recovery mode. (a) Four steps of shape memory cycle are shown: (A) Loading: The sample heated up to $T_{g}+30{ }^{\circ} \mathrm{C}$ and an $80 \%$ compressive strain applied. (B) Fixing: The compressed sample cooled down to $T_{g}-20{ }^{\circ} \mathrm{C}$. (C) Stress Recovery: Sample heated to $T_{g}+30{ }^{\circ} \mathrm{C}$. (D) Unloading: Strain released at $T_{g}+30^{\circ} \mathrm{C}$. This figure is reproduced with permission from [30].

Figure 19: (a) The stress vs. strain behavior of the foams for the 5 cycles. (b) The stress vs. temperature behavior of the foams for 5 cycles. Stages (A), (B), (C) and (D) on plots represent the steps of loading, fixing, stress recovery and unloading respectively. This figure is reproduced with permission from [30].

Figure 20: Shape holding behavior of four H20 foam samples. Four samples named $T_{g}-30, T_{g}, T_{g}+30$ and $T_{g}+60$ are (a) deformed to a $80 \%$ compressive strain at $T_{g}+30{ }^{\circ} \mathrm{C}$ (b) held at specific temperatures of $T_{g}-30$ ${ }^{\circ} \mathrm{C}, T_{g}, T_{g}+30{ }^{\circ} \mathrm{C}$ and $T_{g}+60{ }^{\circ} \mathrm{C}$ respectively for a period of two hours and (c) unloaded at $T_{g}+30^{\circ} \mathrm{C}$. This figure is reproduced with permission from [30].

Figure 21: Demonstration of shape memory behavior of a foam. A $6 \mathrm{~mm}$ cylindrical sample of H60 foam was compressed in a mechanical crimper above its $T_{g}$ and then allowed to cool down to fix the compressed shape. The compressed foam was taped to a rod (seen in the last two panes) and then plunged in a water bath heated to $80^{\circ} \mathrm{C}$. Actuation is seen immediately on contact with water and up to 70 times expansion in volume is observed. Approximate time between panes is 1 second. Scale bar is $2.5 \mathrm{~mm}$. This figure is reproduced with permission from [30].

Figure 22: Molecular structure of HDI and TMHDI, the two isocyanates used in this study. Methyl groups on the backbone of TMHDI were expected to give it a more hydrophobic character relative to HDI.

Figure 23: Structures of the (a) HPED, (b) TEA, (c) water, and (d) excess network units used to calculate the contributions of Hoy's solubility parameter components. This figure is reproduced with permission from [29] 
Figure 24: Cell structure of the foams as obtained from optical microscopy. Images (a), (b), (c), (d), (e) and (f) represent 0TM, 20TM, 40TM, 60 TM, 80TM and 100TM compositions, respectively (scale bar is $500 \mu$ ). This figure is reproduced with permission from [29].

Figure 25: DSC curves of the different foam compositions. Single sharp glass transitions were seen for all compositions within a small range of 63-75 ${ }^{\circ} \mathrm{C}$. This figure is reproduced with permission from [29].

Figure 26: DMTA curves of the different foam compositions. Single sharp glass transitions were seen for all compositions within a small range of 64-80 ${ }^{\circ} \mathrm{C}$. This figure is reproduced with permission from [29].

Figure 27: FTIR curves of the different foam compositions. Changes in the C-H vibrations of the $-\mathrm{CH}_{3}$ groups of TMHDI are evident in the 2800-3000 $\mathrm{cm}^{-1}, 1430-1490 \mathrm{~cm}^{-1}$, and $1350-1400 \mathrm{~cm}^{-1}$ ranges. This figure is reproduced with permission from [29].

Figure 28: Contact angle images of (a) 0TM and (b) 100TM neat/unfoamed polymer compositions. An increase in the contact angle is noticed with an increase in the TMHDI content of the polymer. This figure is reproduced with permission from [29].

Figure 29: Change in the glass transition temperature $\left(T_{g}\right)$ of the foam samples with respect to time submerged in water at $37{ }^{\circ} \mathrm{C}$. The equilibrium $T_{g}$ value is achieved within 5 minutes for all compositions. An increase in the equilibrium $T_{g}$ of the foams is noticed. This figure is reproduced with permission from [29].

Figure 30: Rate of actuation of foam samples of (a) 0TM, (b) 80TM and (c) 100TM compositions. While the OTM foams with only HDI in their composition actuated completely within 5 minutes of exposure to water at $37^{\circ} \mathrm{C}$, the $100 \mathrm{TM}$ foams did not complete their actuation even at 50 minutes. This figure is reproduced with permission from [29].

Figure 31: Ratio of the maximum diameter to the initial/punched diameter as a function of time in $37^{\circ} \mathrm{C}$ water for all foam compositions. Only every fourth error bar is marked for clarity. A decrease in the rate of expansion of the SMP foam is seen with an increase in the TMHDI content. This figure is reproduced with permission from [29].

Figure 32: Molecular structures of the two isocyanates, TMHDI and IPDI, used in the study. 
Figure 33: Summary of the process modifications employed in generation of lower density foams.

Figure 34: Comparison of the volume and dimensional stability of foams from the original process (left), to those from the modified process (right).

Figure 35: Cross section of foam from the original process (left), and the modified process (right). A visible improvement in the foam uniformity is observed.

Figure 36: Foam cell morphology for as-made (right) and reticulated (left) samples, as captured through an optical microscope. Scale bar=500 $\mu$.

Figure 37: Foam cell morphology for reticulated (top) and as-made (bottom) samples, as captured through an optical microscope. Scale bar=500 $\mu$.

Figure 38: Dynamic mechanical analysis of foams for as-made and reticulated samples.

Figure 39: Shape memory behavior of the reticulated foams.

Figure 40: Molecular structures of the polyfunctional hydroxyl monomers used in the synthesis of biodegradable foams.

Figure 41: Cell structure as seen from Scanning Electron Microscopy for a) 0PHDI, b) 25P-HDI, c) 50P-HDI, d) 100P-HDI, e) 100P-TMHDI, and f) 100P-IPDI foam compositions. A mixed open to closed cell morphology, with uniform cell structure is observed for all compositions. Scale bar $=500 \mu$.

Figure 42: DSC curves of various foam compositions show a single $T_{g}$ indicating an amorphous polymer network structure.

Figure 43: FTIR spectra of the samples with varying PCL-t content prior to degradation. Peaks corresponding to the ester, urethane and urea bonds are labeled for identification of respective trends....

Figure 44: FTIR spectra of samples with the varying isocyanate type prior to degradation. Peaks corresponding to the alkyl, ester, urethane, and urea bonds are labeled for identification.

Figure 45: Constrained stress recovery shape memory tests of the 100P-HDI foam samples. The results for five cycles, representing the variation in sample stress with changes in strain and temperature during the loading, fixing, 
stress recovery and unloading steps, are shown in 3D (top) and 2D (bottom) formats.

Figure 46: Mass loss profile of the different foam compositions over the first 28 weeks in $0.1 \mathrm{~N} \mathrm{NaOH}$ media. The fastest mass loss is seen in the $100 \mathrm{P}$ HDI composition, which has the highest PCL-t content and the most hydrophilic isocyanate monomer. The rate of mass loss is observed to decrease with both a decreasing PCL-t content and increasing hydrophobicity of the isocyanate monomer.

Figure 47: FTIR spectra for all the different compositions are shown as a function of time. The wavenumbers of interest, i.e. ester $\left(1730 \mathrm{~cm}^{-1}\right)$, urethane $\left(1695 \mathrm{~cm}^{-1}\right)$, and urea $\left(1640 \mathrm{~cm}^{-1}\right)$ are marked with dotted lines.

Figure 48: Gross morphology of a 100P-HDI composition sample as it degraded over the first 6 weeks. Uniform degradation was observed to occur throughout the bulk of the material, leading to a continuous thinning of the sample, until it became irretrievable at week 7 .

Figure 49: SEM and pathology results of implanted foams demonstrated very promising biocompatibility of the foams. Reproduced with permission from [54].

Figure 50: The four main phases of generation of a foam via the gas-blowing method: Phase I- Mixing of reactive components, Phase II- Foam rise, Phase III- Gelation/Vitrification, and Phase IV- Further cure.

Figure 51: Formation of polyhedral structure of foam cells around the gas bubbles with defined regions of lamella and meniscus (Adapted with permission from [179]).....

Figure 52: A surface tension gradient occurs from the lamella to the meniscus, which leads to the drainage of polymer into the meniscus, thereby thinning the lamella (or the future cell membranes). Adapted with permission from [176].

Figure 53: Preparation of foam sample for DMTA tests.

Figure 54: Structure of Enovate as determined from its name HFC-245fa 


\section{LIST OF TABLES}

Page

Table 1: Summary of composition and key properties of SMP foams available in literature.

Table 2: Summary of the device requirements and design strategy adopted in the synthesis of these materials.

Table 3: Foam formulation details as wt.\% of the final composition. This table is reproduced with permission from [30].

Table 4: Summary of key properties of the foams. This table is reproduced with permission from [30].

Table 5: Results of the biocompatibility study of the foam. This table is reproduced with permission from [30].

Table 6: Composition of foams synthesized using hexamethylene diisocyanate (HDI) and trimethyl hexamethylene diisocyanate (TMHDI) for the isocyanate component of the urethane. This table is reproduced with permission from [29].

Table 7: Composition of neat/non-porous polymers synthesized following the same synthesis scheme as that of the foams (Table 1). Amount of TMHDI was gradually increased from 0TM (all HDI) to 100TM (all TMHDI) composition. No foaming additives such as surfactant, catalysts and physical and chemical blowing agents were added. This table is reproduced with permission from [29].

Table 8: Summary of the key physical properties of the different foam compositions. This table is reproduced with permission from [29].

Table 9: Summary of the results related to the actuation behavior of the foams. This table is reproduced with permission from [29].

Table 10: Hoy's solubility parameters calculated for the different foam compositions. This table is reproduced with permission from [29].

Table 11: Summary of the foam composition. 
Table 12: Summary of foam characterization results before and after plasma treatment.

Table 13: Composition of different foams fabricated in the study. 0P-HDI, 25PHDI, 50P-HDI and 100P-HDI denote an increasing concentration of the biodegradable monomer (PCL-t). 100P-TMHDI and 100P-IPDI denote substitution of HDI in the 100P-HDI composition with an equivalent amount of TMHDI and IPDI respectively.

Table 14: Average foam densities and glass transition temperatures for the different biodegradable and control compositions.

Table 15: Typical values of solubility parameters for some common polymers [158]

Table 16: Calculation of Hoy's solubility parameters for the 100TM composition (Chapter III).

Table 17: Solubility parameters for each structural unit. 190

Table 18: Volume fraction of components using rule of mixtures 190 


\section{CHAPTER I}

\section{INTRODUCTION AND LITERATURE REVIEW}

Last several decades have seen an explosion in the world of polymeric materials that has provided numerous groundbreaking innovations encompassing all walks of life. The understanding that the scientific community has gained from these advances continues to inspire new applications and novel polymeric materials. More recently, advances in a new class of stimuli-responsive shape memory polymers (SMPs) has significantly expanded the material engineering space by introducing materials that are capable of smart physical transformations on remote application of various stimuli.

SMPs are intelligent materials that can be deformed and stored in a temporary/secondary shape, and thereafter can be actuated on demand via an external stimulus, such as heat or ultraviolet light, to return to their primary shape [1-9]. They can be considered to be a subset of a wider group of shape memory materials comprising shape memory ceramics (SMCs) and shape memory alloys (SMAs) [1]. However, compared to these other shape memory materials, SMPs are lightweight, inexpensive, can recover large deformations, and their physical properties can be customized to the end application relatively easily [1,7]. These unique characteristics have led to increased interest in their use in multiple areas including aerospace, civil, electrical, biomedical, and textile industries $[2,7,10]$. Their enormous potential continues to present new applications to the scientific community, with the challenge of designing novel materials spanning the whole spectrum of choice of monomers and their polymerization process, 
to the fabrication of the material with a predefined micro and macro morphology, for achieving a desired set of end physico-mechanical properties. This dissertation is focused on the embolic or regenerative biomedical applications of SMPs. It discusses the development of a novel class of SMP foams tailored for this application, while expanding on the principles of polymer science using a set of novel monomers, customized polymer fabrication processes, and application-specific characterization techniques.

\section{A. Shape Memory Effect}

One of the first reports of SMPs is considered to be a 1941 US patent application

from LB Vernon $[1,11]$. Here he claimed a methacrylic acid ester based dental material that recovered its original shape on heating, owing to an "elastic memory". However, the utility of these materials was not recognized until the 1960s, when heat shrinkable films and tubings were commercialized using covalently crosslinked polyethylene [11-15]. Towards the beginning of 1980 s, the research in shape memory polymers began to pick up and there has been a near exponential rise in the number of publications on this topic since then (Figure 1) [1]. Several comprehensive review papers are available on the mechanism of shape memory behavior in polymers $[1-6,10,16]$. These materials present unique advantages over shape memory alloys such as light weight, large shape recovery of up to $400 \%$ plastic strain, non-toxicity, non-mutagenicity, ease of processing, low cost and biodegradability [1, 3, 7]. Further, a significant level of customizability of the material mechanical properties is possible with polymers, and multiple chemical 
formulations for SMPs have already been reported with a wide range of mechanical properties [1]. This section reviews the basis of shape memory behavior, the broad chemical classes of SMPs and the SMP foams, and the characteristic thermomechanical cycle for shape memory characterization.

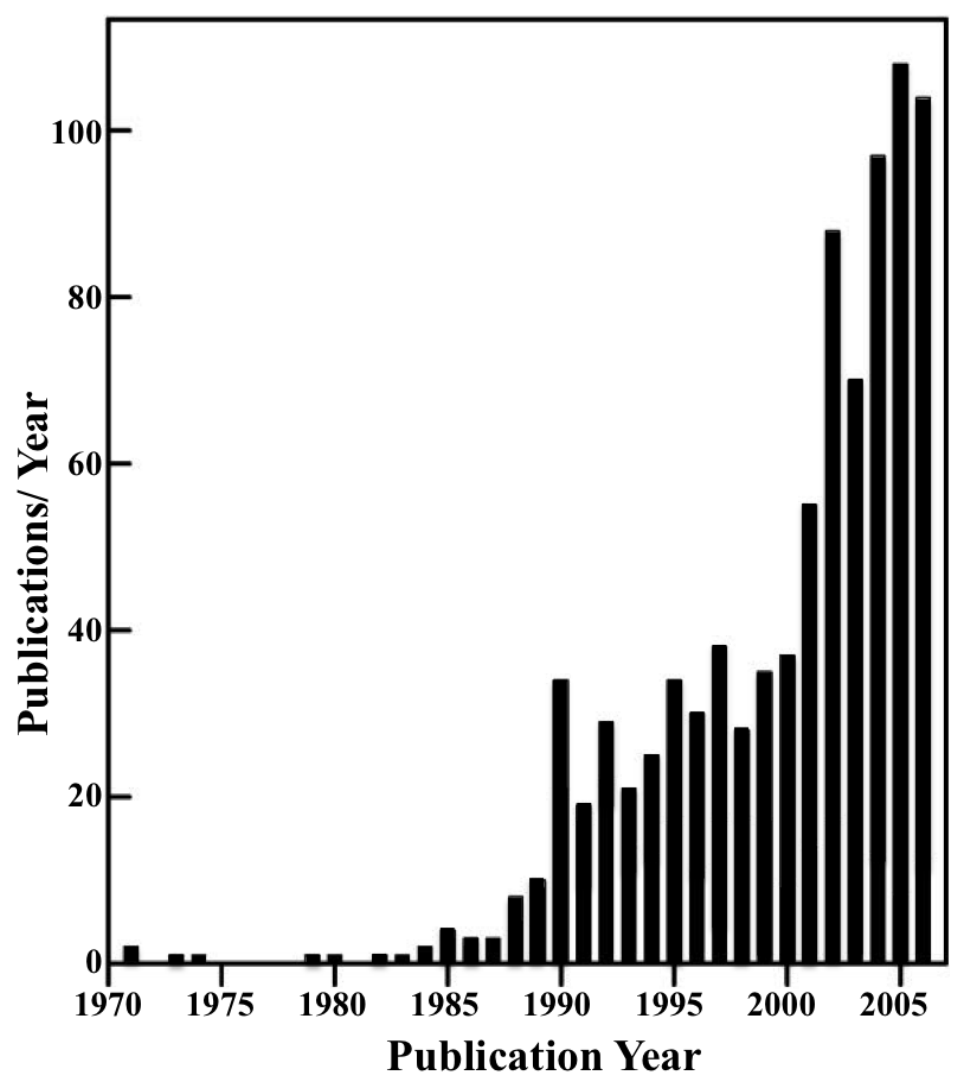

Figure 1: The volume of SMP research has seen a near exponential rise in the recent decades. This figure is adapted with permission from [1].

\section{Basis of Shape Memory}

Nearly all polymer chains have an intrinsic ability to exhibit some shape memory behavior due to their propensity to attain high entropy - low energy configuration, and 
the lack/availability of energy to achieve this transformation. Briefly, the composition of the polymer chains and their physical structure defines a characteristic transition temperature $\left(T_{\text {trans }}\right)$ of the polymer. Only a limited vibrational molecular motion is possible below this temperature. As the polymer is heated to this transition temperature, the polymer chains gain enough energy that a long range co-ordinated molecular motion can set in. The polymer experiences a drop in modulus, reaching the rubbery state, beyond this $T_{\text {trans }}$; and may eventually flow or thermally decompose at still higher temperatures, depending on whether it is physically or covalently crosslinked.

For the shape memory behavior, the polymer is physically deformed to a temporary shape while in its rubbery state, and this temporary shape is effectively frozen in by vitrification or crystallization, as the material in deformed state is cooled to below the $T_{\text {trans }}$. The polymer maintains the deformed shape in its glassy state as the polymer chains do not have sufficient energy to attain their preferred high entropy, low energy primary state. Physical or chemical crosslinks between the polymer chains act as net-points or anchors that further restrict the chain motion or conformational reorganization in this state. As soon as sufficient thermal energy is provided to the polymer chains, by heating to the $T_{\text {trans }}$, they recover their preferred high entropy, low energy conformation, and thereby the macroscopic primary shape of the polymer. When this shape memory effect is specially customized to, or is exploited in an application, the polymer systems are referred to as SMPs. It is to be noted that this dissertation discusses the thermally actuated polymers, but other stimuli such as ultraviolet light and $\mathrm{pH}$ have also been shown to elicit shape memory behavior in polymers $[17,18]$. 


\section{Classes of SMPS}

As described in the previous section, the chemical structure of polymers can be customized to obtain a functionally useful shape memory effect. Briefly four major categories of the chemical structure of thermally actuated SMPs have been identified [1]:

\subsection{Covalently Crosslinked Glassy Thermosetting Polymers (Class I)}

These materials represent the non-crystallizing amorphous thermoset polymers that undergo a glassy to rubbery transition across a characteristic glass transition temperature $\left(T_{g}\right)$. The shape memory behavior is invoked across this $T_{g}$, i.e. molding of the material to the temporary/secondary shape, and its fixing/storage take place above and below this $T_{g}$ respectively. In addition to thermoset materials, linear polymers of

ultra high molecular weight $\left(>10^{6} \mathrm{~g} \cdot \mathrm{mol}^{-1}\right)$ with significant number of entanglements (>25) per chain may also show limited flowability above $T_{g}$, and are placed in this class of SMPs. Polymers such as epoxy and polynorbornene fall in this category of SMPs.

\subsection{Covalently Crosslinked Semi-crystalline Polymers (Class II)}

Similar to the Class I materials, the permanent shape of these materials is established by chemical crosslinks. However, they also contain crystalline regions, which offer the melting temperature of the crystallites as an alternative temperature to invoke shape memory, aside from the $T_{g}$. Chemically crosslinked trans-polyisoprene and polycyclo-octene, and electron beam crosslinked polyethylene are some examples of this category of SMPs. 


\subsection{Physically Crosslinked Glassy Copolymers (Class III)}

Unlike the covalently crosslinked materials, physically crosslinked materials offer simpler processability as they can be remolded to a desirable primary shape in the flow regime of the polymer. This class of materials are typically a copolymer of two or more type of repeating units, and undergo a microphase separation, constituting a physically crosslinked rigid amorphous or crystalline "hard domain" featuring a $T_{g}$ or melting temperature $\left(T_{m}\right)$ respectively, and an amorphous "soft domain" featuring a $T_{g}$. Shape memory is invoked across the $T_{g}$ of the soft domain. The primary shape of the material can be redefined by heating the polymer above the $T_{m}$ or $T_{g}$ of the hard domain. Typical examples of this class of SMPs include a blend of thermoplastic polyurethane with polycaprolactone segments and phenoxy resin, and copolymerization of polyhederal oligosilsesquioxane with norbornene.

\subsection{Physically Crosslinked Semi-crystalline Copolymers (Class IV)}

Similar to the Class III materials, Class IV SMPs can be remolded to a different primary shape above the melting temperature of the "hard domain". However, the "soft domain" undergoes crystallization as well, and its melting temperature functions as the transition temperature for the shape memory behavior of the material. Triblock copolymers of styrene-trans butadiene-styrene and multiblock polyurethanes with semicrystalline soft segments e.g. poly ethylene oxide constitute this category of SMPs. 


\section{SMP Foams}

Processing SMPs into a porous form further increases the applications of these smart materials due to their unique properties including high thermal and electrical insulation, high volume changes on recovery from compressive strain (i.e. low storage volume in compressed state), and low density. Multiple SMP foams have been developed and are suggested for use in numerous commercial applications (Table 1). Although some SMP foams are proprietary, the following general chemical classes of SMP foams have been identified in the literature (Table 1) [19].

\subsection{Polyurethanes}

Polyurethanes are typically physically crosslinked polymers obtained from the reaction of an isocyanate with polyether or polyester type polyols, and generally fall in Class III SMPs, i.e. physically crosslinked amorphous SMPs [20-26]. The hard segments comprising physically crosslinked isocyanates provide the netpoints for shape memory, and the shape fixing is achieved by vitrification of the material below the $T_{g}$ of the soft segment. However when a crystallizable soft segment is used, such as pentanedecalactone or polycaprolactone [27, 28], the soft segment exhibits a melting transition and its $T_{m}$ is used as the transition temperature for shape fixing; these fall in the Class IV SMPs with a physically crosslinked crystalline structure. It is to be noted here that Chung et al. used a trifunctional polycaprolactone triol $\left(\mathrm{M}_{\mathrm{w}} 4000 \mathrm{~g}\right)$, in their study [27]. It showed a melting transition in this polymeric system, but may exhibit a significantly reduced tendency to crystallize at lower molecular weights of the 
polycaprolactone triol. Another family of covalently crosslinked amorphous Class I Polyurethane SMP foams has also been reported by Singhal et al. [29, 30]. These materials claim a high density of covalent crosslinks with use of low molecular weight branched monomers and are discussed in detail in this dissertation.

\subsection{Polystyrene}

Polystyrene typically forms a rigid amorphous Class I type thermoset SMP, although it has been used as a hard domain of a physically crosslinked glassy copolymer with trans-polybutadiene [1]. The polystyrene based SMP foams, named Veriflex (Cornerstone Research Group), use styrene, divinyl benzene and/or vinyl neodecanoate as raw materials [31-36]. In these systems, benzoyl peroxide or dibenzoyl peroxide have been used as radical initiators for polymerization, in combination with dichlorodimethyl silane, possibly to facilitate the block copolymer formation via living chain polymerization. Also, tricresyl phosphate and Closite 20A have been used in varying amounts as plasticizer and reinforcing filler respectively [35, 36]. While they are proprietary, these polymeric systems show a possibility of developing Class I thermoset amorphous systems, as with styrene and divinyl benzene with varying amounts of plasticizer/filler to modify the transition temperature, or a physically or chemically crosslinked copolymer with vinyl neodecanoate $\left(T_{m} \sim 25^{\circ} \mathrm{C}\right)$, that may exhibit a melting transition (Class III or Class II SMPs). 
Table 1: Summary of composition and key properties of SMP foams available in literature.

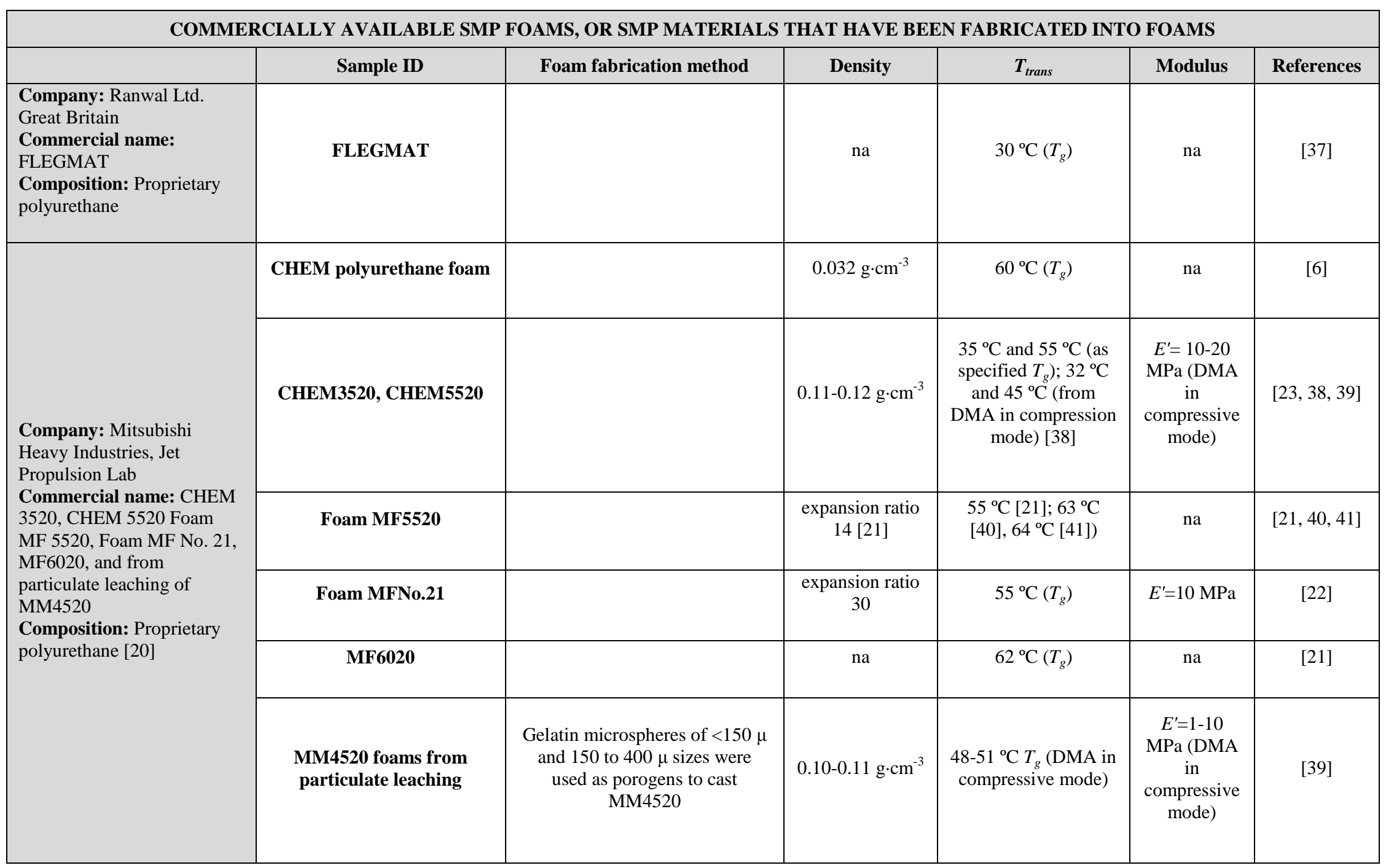


Table 1 continued.

\begin{tabular}{|c|c|c|c|c|c|c|}
\hline & Sample ID & Foam fabrication method & Density & $T_{\text {trans }}$ & Modulus & References \\
\hline \multirow{2}{*}{$\begin{array}{l}\text { Company: Composite } \\
\text { Technology Development } \\
\text { Commercial name: DP5.1 } \\
\text { and TEMBO 3XE ( } 2 \text { part } \\
\text { thermoset epoxies) } \\
\text { Composition: Proprietary }\end{array}$} & DP5.1 & $\begin{array}{c}\text { Magsilica (Degussa Corp.) and } \\
\text { nano-magnetite particles (Sigma } \\
\text { Aldrich Inc.) were used for foam } \\
\text { fabrication [42] }\end{array}$ & $\begin{array}{l}0.164-0.218 \\
\mathrm{~g} \cdot \mathrm{cm}^{-3} ; 15-20 \% \\
\text { relative density } \\
\quad[42]\end{array}$ & $\begin{array}{c}\sim 65-85^{\circ} \mathrm{C}(\mathrm{DMA} \text { in } \\
\text { tensile mode) }[42]\end{array}$ & $\begin{array}{c}E^{\prime}=80-100 \\
\text { MPa, DMA } \\
\text { in tensile } \\
\text { mode[42] }\end{array}$ & {$[42,43]$} \\
\hline & TEMBO 3XE & & $\begin{array}{l}20 \%, 30 \%, 40 \% \\
\text { relative density }\end{array}$ & $\begin{array}{l}\text { 83- } 88^{\circ} \mathrm{C} \text { (DMA in } \\
\text { tensile mode) }\end{array}$ & $\begin{array}{c}E^{\prime}=\sim 60-120 \\
\text { MPa (DMA } \\
\text { in tensile } \\
\text { mode) }\end{array}$ & [44-46] \\
\hline \multirow{2}{*}{$\begin{array}{l}\text { Company: Cornerstone } \\
\text { Research Group } \\
\text { Commercial Name: } \\
\text { Veriflex } \\
\text { Composition: } 2 \text { part } \\
\text { thermoset resin system- Part } \\
\text { A has styrene and/or divinyl } \\
\text { benzene and/or vinyl } \\
\text { neodecanoate; Part B has } \\
\text { hardener based on benzoyl } \\
\text { peroxide and/or dibenzoyl } \\
\text { peroxide, tricresyl phosphate } \\
\text { and dichlorodimethyl silane } \\
\text { (other details proprietary) }\end{array}$} & $\begin{array}{l}\text { Syntactic foam from } \\
\text { Veriflex using glass } \\
\text { microballoons and MW } \\
\text { carbon nanotubes }\end{array}$ & $\begin{array}{l}\text { Microballoons (Potter's } \\
\text { Industry) and/or MW Carbon } \\
\text { Nanotubes (Cheap Tubes Inc.) } \\
\text { were used to generate foams } \\
\text { from the Veriflex epoxy resin }\end{array}$ & $\begin{array}{c}40 \text { vol. } \% \\
\text { microballoons, } \\
0.15 \text { vol. } \% \text { MW } \\
\text { carbon } \\
\text { nanotubes [47- } \\
\text { 49]; } 40 \text { vol. } \% \\
\text { microballoons } \\
{[31-34]}\end{array}$ & $\begin{array}{l}62^{\circ} \mathrm{C}\left(T_{g}, \text { for } \mathrm{SMP}\right) \\
\text { [47-49]; } 71{ }^{\circ} \mathrm{C} T_{g} \\
\text { (DMA in single } \\
\text { cantilever beam } \\
\text { mode); } 64^{\circ} \mathrm{C} T_{g} \\
\text { (DMA in single } \\
\text { cantilever beam } \\
\text { mode) [31-34] }\end{array}$ & $\begin{array}{c}E^{\prime}=1.24 \mathrm{GPa} \\
\text { (for SMP } \\
\text { only) [47- } \\
49] ; 845 \\
\text { MPa (DMA } \\
\text { in single } \\
\text { cantilever } \\
\text { beam mode) } \\
\text { [31-34] }\end{array}$ & $\begin{array}{c}{[31-34,47-} \\
49]\end{array}$ \\
\hline & $\begin{array}{c}\text { Composite of Veriflex and } \\
\text { a dimethyl dehydrogenated } \\
\text { tallow quaternary } \\
\text { ammonium organoclay } \\
\text { Closite 20A (Southern Clay } \\
\text { Products) } \\
\end{array}$ & $\begin{array}{c}\text { Foam fabricated by supercritical } \\
\mathrm{CO}_{2} \text { saturation (at } 500,600,700, \\
800 \text { psi pressures) at } 50{ }^{\circ} \mathrm{C} \text {, for } \\
0,5,10 \text {, and } 15 \text { seconds }\end{array}$ & $\begin{array}{l}\text { Relative density } \\
\text { of } 30 \text { to } 90 \% \\
\text { based on } \\
\text { foaming } \\
\text { variables }\end{array}$ & $\begin{array}{c}62{ }^{\circ} \mathrm{C} T_{g} \text { for SMP (as } \\
\text { provided) }\end{array}$ & na & {$[35,36]$} \\
\hline $\begin{array}{l}\text { Company name: Polymer } \\
\text { Technology Group, Berkeley } \\
\text { Commercial Name: } \\
\text { CaloMER } 48 \\
\text { Composition: Proprietary }\end{array}$ & $\begin{array}{l}\text { CaloMER } 48 \text { foams using } \\
\text { foaming agents }\end{array}$ & $\begin{array}{l}\text { Blowing agents Plastronfoam } \\
\text { BSH (based on } \mathrm{NaHCO}_{3} \text { and } \\
\text { citric acid derivatives) and W16 } \\
\text { (polymer microspheres } \\
\text { encapsulating low boiling point } \\
\text { liquid) (FerroPlast S.r.l.) were } \\
\text { used; } 30 \% \text { w/w of BSH (C48) } \\
\text { and } 12 \% \text { w/w of W16 (C48) } \\
\text { were extruded to achieve foams }\end{array}$ & $0.85-0.91 \mathrm{~g} \cdot \mathrm{cm}^{-3}$ & $\begin{array}{l}61-89^{\circ} \mathrm{C} T_{g}(\mathrm{DMA} \text { in } \\
\text { tensile mode) }\end{array}$ & $\begin{array}{l}E^{\prime}=\sim 1 \mathrm{GPa} \\
\text { (DMA in } \\
\text { tensile } \\
\text { mode) }\end{array}$ & [39] \\
\hline
\end{tabular}


Table 1 continued.

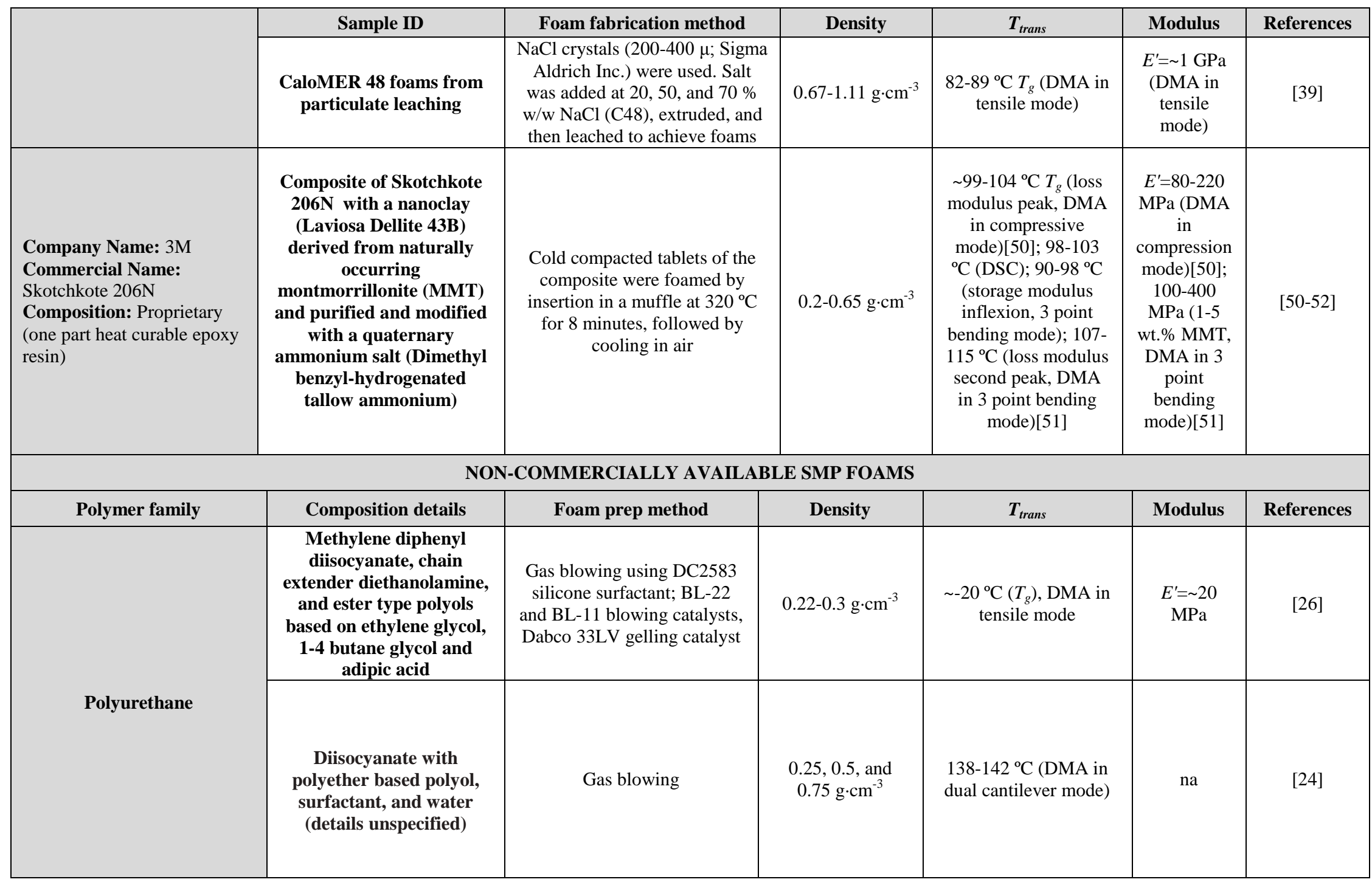


Table 1 continued.

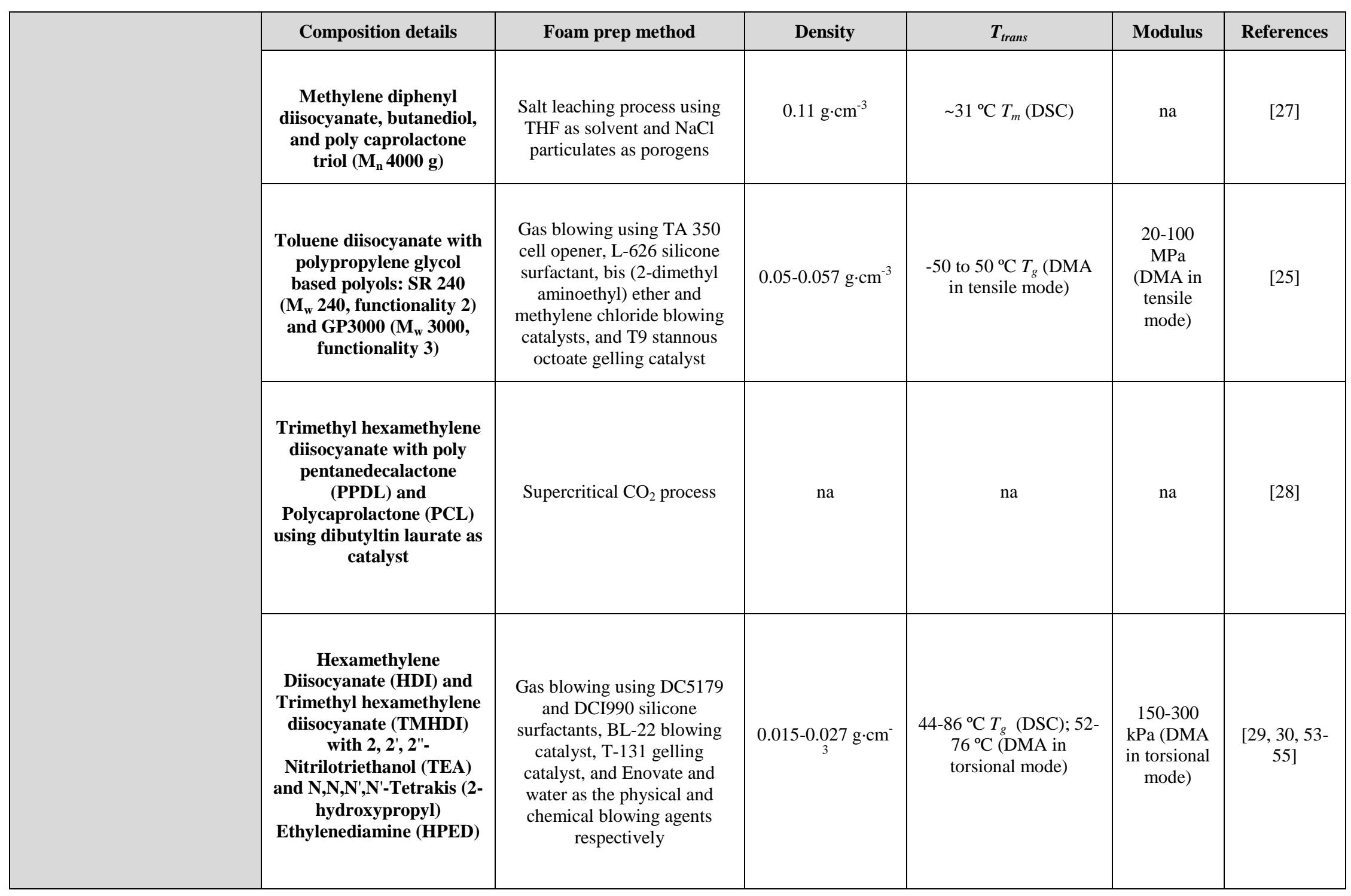


Table 1 continued.

\begin{tabular}{|c|c|c|c|c|c|c|}
\hline Polymer family & Composition details & Foam prep method & Density & $T_{\text {trans }}$ & Modulus & References \\
\hline Hybrid organic-inorganic & $\begin{array}{c}\text { (3-aminopropyl) } \\
\text { terminated poly } \\
\text { (dimethylsiloxane) was } \\
\text { reacted with } \\
\text { \&-caprolactone in a ring } \\
\text { opening polymerization } \\
\text { with stannous } \\
\text { 2-ethylhexanoate. This } \\
\text { was then reacted with } \\
\text { acryloyl chloride to yield } \\
\text { AcO-PCL40-b-PDMS37- } \\
\text { b-PCL40-OAc }\end{array}$ & $\begin{array}{c}\text { Particulate leaching using } \\
\mathrm{NaCl} \text { particles }(181 \pm 29 \mu, \\
268 \pm 35 \mu \text { and } 459 \pm 69 \mu) \text {. } \\
\text { Partially fused particulate } \\
\text { leaching method was } \\
\text { employed for better pore } \\
\text { interconnectivity }\end{array}$ & $\begin{array}{l}72-86 \% \text { porosity; } \\
0.84-0.96 \mathrm{~g} \cdot \mathrm{cm}^{-3}\end{array}$ & $\begin{array}{c}\sim 40^{\circ} \mathrm{C} T_{g} \text { (method not } \\
\text { specified) }\end{array}$ & na & {$[56]$} \\
\hline \multirow[t]{2}{*}{ Polycaprolactone } & $\begin{array}{c}\text { Functionalized } \\
\text { polycaproplactone matrix }\end{array}$ & $\begin{array}{l}\mathrm{NaCl} \text { particulates were mixed } \\
\text { in polycaprolactone and the } \\
\text { polymer was crosslinked with } \\
\text { tetrathiol to lock in the } \\
\text { porous state. After } \\
\text { crosslinking, salt particles } \\
\text { were extracted using water }\end{array}$ & na & na & na & {$[57]$} \\
\hline & $\begin{array}{l}\text { Covalently crosslinked } \\
\text { polycaprolatone matrix } \\
\text { reinforced with } \mathrm{MW} \\
\text { carbon nanotube filler } \\
\text { coated with } \mathrm{Fe}_{3} \mathrm{O}_{4} \\
\text { nanoparticles }\end{array}$ & Electrospinning & na & na & na & {$[58]$} \\
\hline Polystyrene & $\begin{array}{l}\text { Styrene based resin, } \\
\text { hardener and a vesicant } \\
\text { (details unspecified) }\end{array}$ & $\begin{array}{l}\text { na (components were mixed } \\
\text { and cured) }\end{array}$ & na & $70^{\circ} \mathrm{C} T_{g}$ (DSC) & na & [59] \\
\hline
\end{tabular}




\subsection{Crosslinked Caprolactone}

A SMP based on Tetrathiol crosslinked polycapolactone matrix was reported by Baker et al. [57]. Another related chemically crosslinked polycaprolactone matrix, further reinforced with carbon nanotube fillers was reported by Gong et al. [58]. These systems are expected to form Class II crystalline thermoset SMPs, with the melting of polycaprolactone crystalline segments demarcating the loss of the shape fixing phase. Such molecular structures form viable SMP systems; however, a limitation is that a low control over the molecular weight between crosslinks hinders the formation of crystalline segments of uniform size. A broad distribution of crystal size is thereby achieved, and may lead to a broad transition range. Hence use of copolymers is often

preferred in such systems. This strategy helps in the formation of uniform length of crystalline segments, leading to sharp and narrow transitions [1].

\subsection{Epoxy}

Foams made from proprietary thermoset SMP epoxy resins, comprising two part in situ reactive system, as in DP5.1 and TEMBO 3XE (Composite Technology Development) [42-45], and a one part heat curable thermoset system, Skotchkote 206N (3M Company) [50-52], have been used in multiple investigations. These systems form a Class I type chemically crosslinked glassy networks, and employ material $T_{g}$ as the shape fixing transition temperature. The heat curable one part epoxy systems offer a unique advantage in generation of SMP foams; these materials can be subjected to 
controlled air turbulence, as by insertion in a muffle, in the presence of high temperatures, leading to facile synthesis of porous structures [50-52].

\subsection{Hybrid Organic-inorganic}

A hybrid organic-inorganic photo-crosslinked shape memory foam based on polydimethyl siloxane and polycaprolactone segments was reported by Zhang et al. [56]. These materials exhibit a crystalline shape fixing phase of Polycaprolactone in a cocontinuous crosslinked matrix of silicone rubber, and fall in the Class II SMP materials according to the classification by Liu et al. [1]. The synthesis process demonstrated a high control on the lengths of polycaprolactone and polydimethyl siloxane segments, and these could be precisely varied for modulating the material's mechanical properties.

Several methods of foam fabrication have been used for SMP materials (Table 1), including particulate leaching or compacting, gas blowing, super critical $\mathrm{CO}_{2}$ saturation, electrospinning, and thermal curing in induced air currents. Chapter II discusses the various foaming methods, relative to the materials discussed in this dissertation. However, readers are recommended to other in-depth reviews on foam fabrication methods for a detailed description of each process $[19,60]$.

\section{Thermomechanical Cycle}

The thermomechanical shape memory cycle is fundamental to the understanding and characterization of SMPs, and is briefly discussed here. Following are the primary 
steps involved in programming an SMP through the thermomechanical cycle:

\subsection{Loading}

Heating the sample to $T>T_{\text {trans }}$ and application of strain to reach the temporary/deformed shape.

\subsection{Fixing}

Cooling the sample to $T<T_{\text {trans }}$ while maintaining the applied strain.

\subsection{Recovery}

The temporary/deformed shape, thus fixed, can be recovered in two ways:

\subsubsection{Constrained Stress Recovery}

The constrained sample is heated back up to $T>T_{\text {trans }}$ while maintaining the applied strain. Finally unloading of the sample is done at $T>T_{\text {trans }}$ to recover the primary shape.

\subsubsection{Free Strain Recovery}

The sample is heated to $T>T_{\text {trans }}$ while maintaining a zero stress condition, and the recovery of the primary shape is achieved simultaneously with the temperature rise.

Laboratory characterization of the shape memory behavior of SMPs in a common dynamic mechanical analyzer, utilizes the controlled stress and/or stain conditions to perform this 3-4 step thermomechanical cycle. This cycle can be graphically depicted in 
a 3-D plot of temperature-stress-strain as shown in Figure 2 for an SMP foam test in compressive mode. The first step is the "loading" or compression of the material at elevated temperatures $\left(T>T_{\text {trans }}, T_{\text {high }}\right)$. As the degree of strain increases, a concomitant increase in the stress is recorded. At the molecular level, this increase in stress can be related to the resistance to deformation exerted by polymer chains in order to maintain their high entropy, low energy primary shape at $T_{h i g h}$. After the desired level of strain is applied on the material, this strain is "fixed" by cooling to $T<T_{\text {trans }}\left(T_{\text {low }}\right)$. As this step is performed while maintaining the strain, a drop in the stress is observed. The decrease in the thermal energy of the polymer chains limits their molecular motion, leading to a drop in the macroscopic stress that can be applied (on restraining platens) in this state of reduced mobility. After this programming of the temporary shape, the primary shape can be recovered by either a) free strain recovery $\left(\mathrm{C}_{\mathrm{i}}\right)$, where the temperature is increased to $T_{\text {high }}$ while maintaining a zero stress condition allowing simultaneous recovery of the strain, or b) constrained stress recovery $\left(\mathrm{C}_{\mathrm{ii}}\right)$; here the temperature is increased to $T_{\text {high }}$ while maintaining the strain levels, allowing the stress to rise with the temperature as polymer chains gain molecular mobility and try to recover the primary shape. The final step of strain recovery, "unloading", is performed after $\mathrm{C}_{\mathrm{ii}}$ to allow the sample to recover its primary shape. In either case $\left(\mathrm{C}_{\mathrm{i}}\right.$ or $\left.\mathrm{C}_{\mathrm{ii}}\right)$, the sample recovers the primary high entropy, low energy state when both the conditions of $T>T_{\text {trans }}$, and no external stress are met. $\varepsilon_{\text {pre }}$ and $\varepsilon_{\text {rec }}$, in Figure 2, depict the initial and recovered values of strains. By this definition, \% shape recovery of the SMP can be calculated as $\left(\varepsilon_{\text {rec }} / \varepsilon_{\text {pre }}\right) \cdot 100$. 


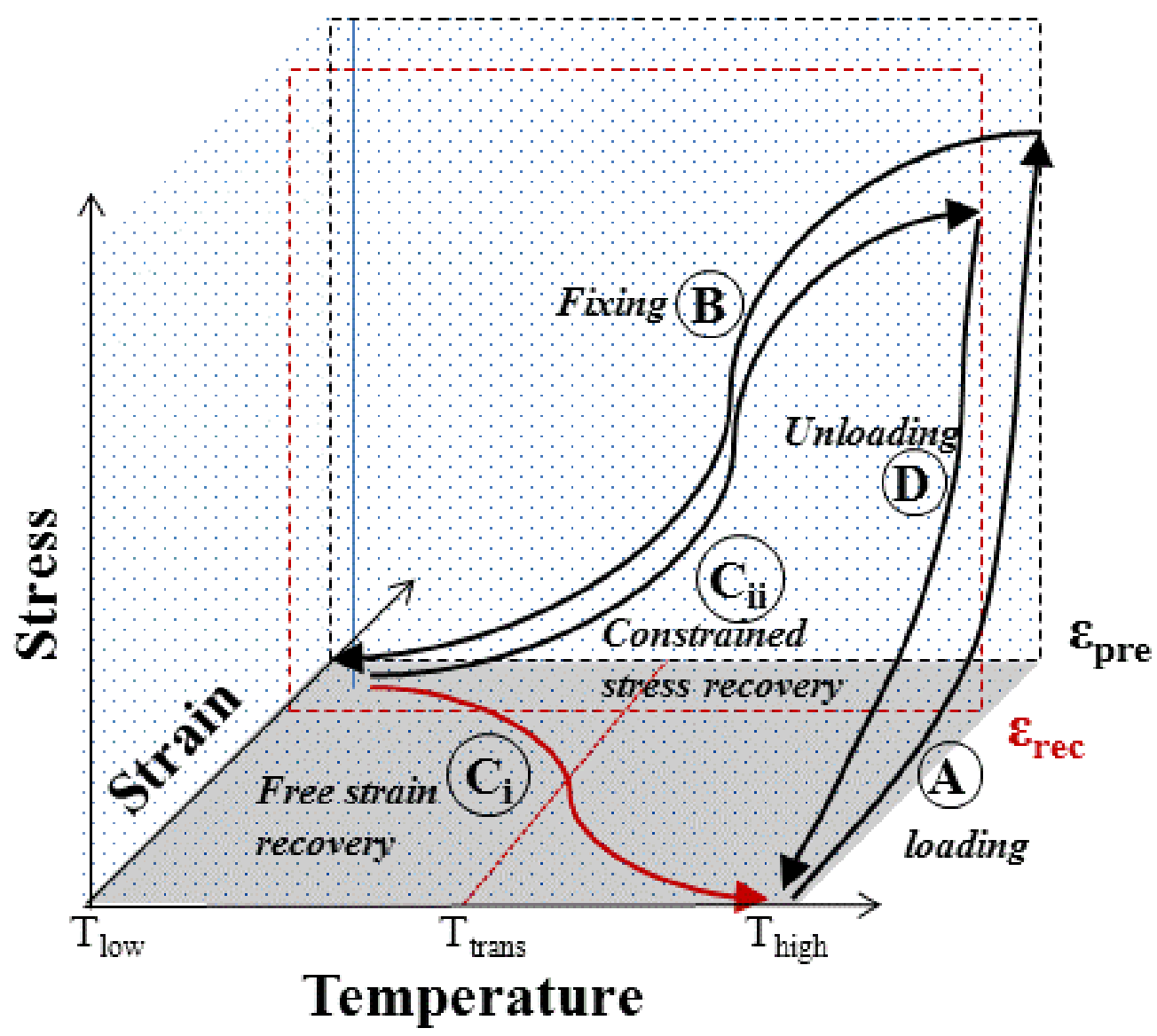

Figure 2: Schematic of the thermomechanical cycle for shape memory polymers in compression mode. Large compressive strain is imposed along A (Loading), the SMP is cooled along $B$ to lock in the temporary compressed shape (Fixing), the material is then allowed to recover either by free strain recovery along $C_{i}$, or by constrained stress recovery along $C_{i i}$. A final step of unloading along $D$ is performed after the constrained stress recovery $C_{i i}$. 


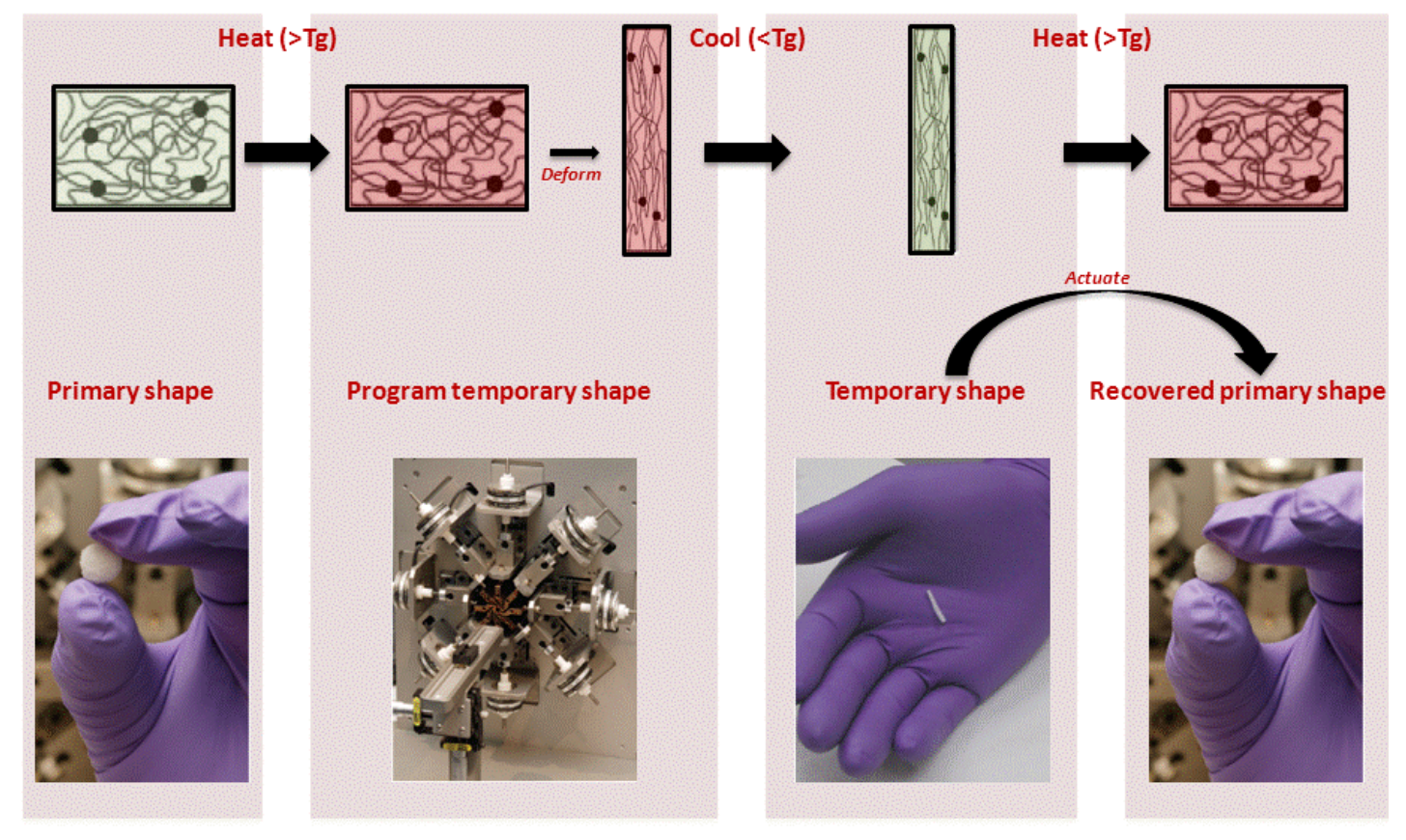

Figure 3: Schematic representation of changes in the polymer chain orientation, and the physical shape of a SMP foam as it is taken through the thermomechanical cycle. Image courtesy: Dr. Thomas S. Wilson [demonstration of the programming and physical shape transformations of a SMP foam device in the bottom panel].

Figure 3 shows the physical transformations of SMP foam as it is taken through the shape memory cycle. It is this unique property of SMP foams of undergoing a large volume change on actuation that renders them useful in numerous practical applications.

\section{B. Applications of SMP Foams}

Owing to their unique properties, SMP foams have been proposed in a wide range of applications, ranging from biomedical and aerospace, to civil and textile industries. This section gives an overview of the applications of SMP foams suggested in the literature [19]. 


\section{Biomedical Applications}

Numerous reviews of biomedical applications of SMPs have been published [8, $10,61,62]$. Some primary areas of biomedical applications of porous SMP foams include embolic devices for filling vascular lesions, regenerative scaffolds for filling bone defects, haemostatic sponges, and soft tissue scaffolds. Also combination devices of SMPs, functioning as drug delivery, or gene therapy platforms have been suggested.

SMP foams from Mitsubishi Inc. have been widely investigated for vascular embolic applications. Metcalfe et al. were the first to publish about the strong embolic potential of these materials with no cytotoxicity or mutagenicity, and a good in vivo healing response [43]. Also, in other tests by DeNardo et al., good cytocompatibility was observed for Mitsubishi CHEM3520 and CHEM5520 foams [23, 38].

Another class of highly covalently crosslinked polyurethane SMP foams from our group has been actively developed for the vascular embolic applications during the last decade [30], and will be discussed in detail in this dissertation. Papers demonstrating the critical properties of these materials, such as their biocompatibility through in vivo porcine implants [54], and safety and efficacy of the implantation process through applied stress estimations [63] have been published.

Several proof-of-concept studies have been performed to understand the application of SMP foams for embolization of vascular lesions. For instance, a magnetic resonance imaging based characterization of fluid flow through a foam sample demonstrated a slow and tortuous flow profile [64]. A computational modeling of the local temperatures and flow profiles in the vasculature before and after the actuation of 
the foam device explored the potential of thermal damage during implantation [65]. Finally, a combination device using SMP foam and a neat SMP stent was proposed for an improved treatment of the wide-necked fusiform aneurysms [66].

\section{Aerospace Applications}

Deployable structures with low mass and low packaged volume are always attractive to aerospace applications for maximizing fuel efficiency and improving aircraft performance. SMP foams, with their property of large volume expansion on actuation, in addition to being low cost and reliable, hence present a very inviting territory for aerospace applications. Also, the SMP foams have been thought to effectively absorb impact forces and better sustain contact with any striking objects [67]. Further, for their actuation in such applications, conversion of solar energy to thermal energy using a removable thermal blanket, has been proposed as a facile and viable mechanism [67].

Use of SMP foams has been demonstrated in several small laboratory-scale concepts such as mini nanorover wheels $(\sim 6 \mathrm{~cm})$ [67], and foldable microcars [41]. But, there is an increasing interest in their use in larger aerospace structures. For e.g. large boom structures ( 50 meters long), comprising truss elements using SMP foam and carbon fiber reinforced polymers have been developed [67]. Also, large solar sails, ten to several hundred meters in dimension, using thin films of microcellular SMP foams, are being considered for propelling spacecrafts [68]. Adaptive airplane wings, for improved performance and fuel efficiency, constitute another popular area of application of SMP 
foams [69]. Tests using SMP foams as substitute to heavyweight actuation machinery in low speed small planes, have already been shown to produce noticeable changes in the flight performance [70]. Although there have been some disappointments [69], use of SMP foams demonstrate a huge potential in aerospace applications.

\section{Robotic Grips}

SMP foams have been investigated for use in Robotics. Monkman and coworkers reported the use of commercially available polyurethane-based SMP foam (FLEGMAT, Ranwal Ltd) in an innovative robotic gripping technology for transportation of fragile objects [37]. SMP foams show a large variation in their compliance across the transition temperature. Above the $T_{g}\left(\sim 30^{\circ} \mathrm{C}\right.$ for FLEGMAT), the foam is compliant enough to conform to irregular geometries of an object to be gripped. This can allow gripping of delicate objects without damaging them. However, below the $T_{g}$ foam's compliance is greatly reduced, such that the foam remains firm, permitting safe transportation of the object [37].

\section{Self-healing Applications}

Sandwich panels with a foam core are employed in a wide range of structural applications, such as concrete pavement or bridge deck. They typically use syntactic foams fabricated by dispersing microballoons, or small glass spheres into polymeric matrices. There has been a recent interest in the development of self-healing technology allowing self-repair of structural damages to these constructions, by means of the shape 
memory technology [47]. Li and coworkers proposed a two-step "close then heal" approach to this kind of self-healing [31]. This process utilizes SMP based foam to close or fill damaged areas, such as cracks or voids, as described in Figure 4. After the foam is damaged, confined shape recovery is induced via a temperature rise causing the SMP foam to expand into and fill any cracks or voids that may have formed. It is often also coupled with a healing mechanism, such as microcapsules containing a material that can polymerize upon release.

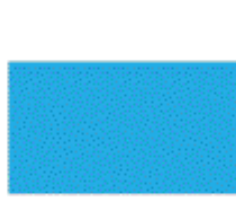

(a) Permanent Shape B after curing $(T \& T)$

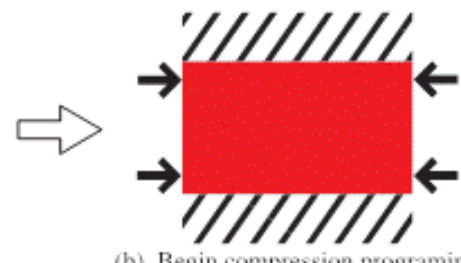

by Begit eomprexsion pegraming with sonfinement $(T \mathrm{~T}$ )

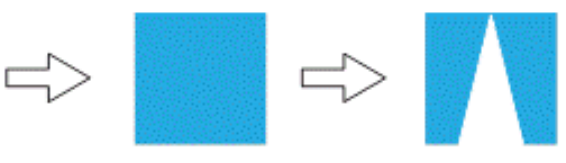

(e) After pogramming in working shape ishape Al. $\left(\mathrm{T}<\mathrm{T}^{\mathrm{g}} \mathrm{g}\right.$ (d) Cracking exused by impast. (T⿺𠃊)

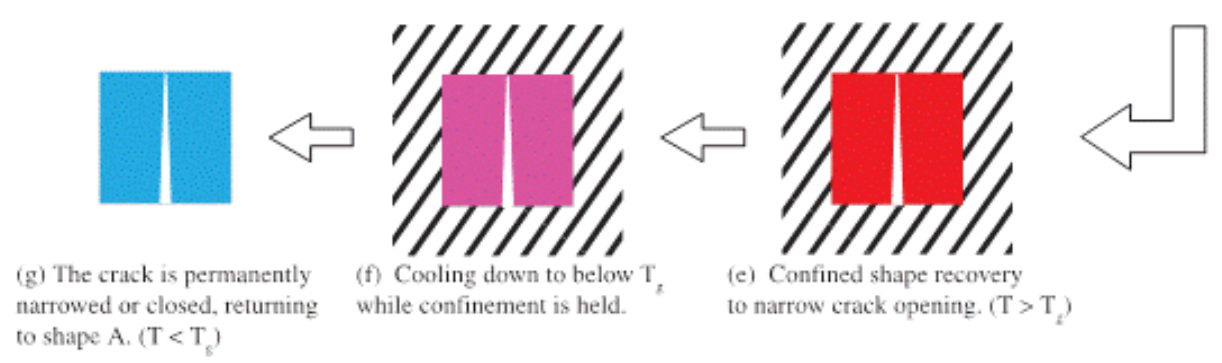

Figure 4: Schematic of the self-healing scheme of the proposed smart foams $\left(T_{g}\right.$ is the transition temperature of the foams). The figure is reproduced with permission from [31].

The effectiveness of this process can be seen in Figure 5 which shows an ultrasonic c-scan of a panel after each of seven impact and healing cycles [47]. Because this process is heavily reliant on confinement, a 2D grid is typically integrated into the 
foam. This, along with the confinement in the $3^{\text {rd }}$ direction by the face sheets, has been shown to give increased healing efficiency and strength to the overall structure [49].

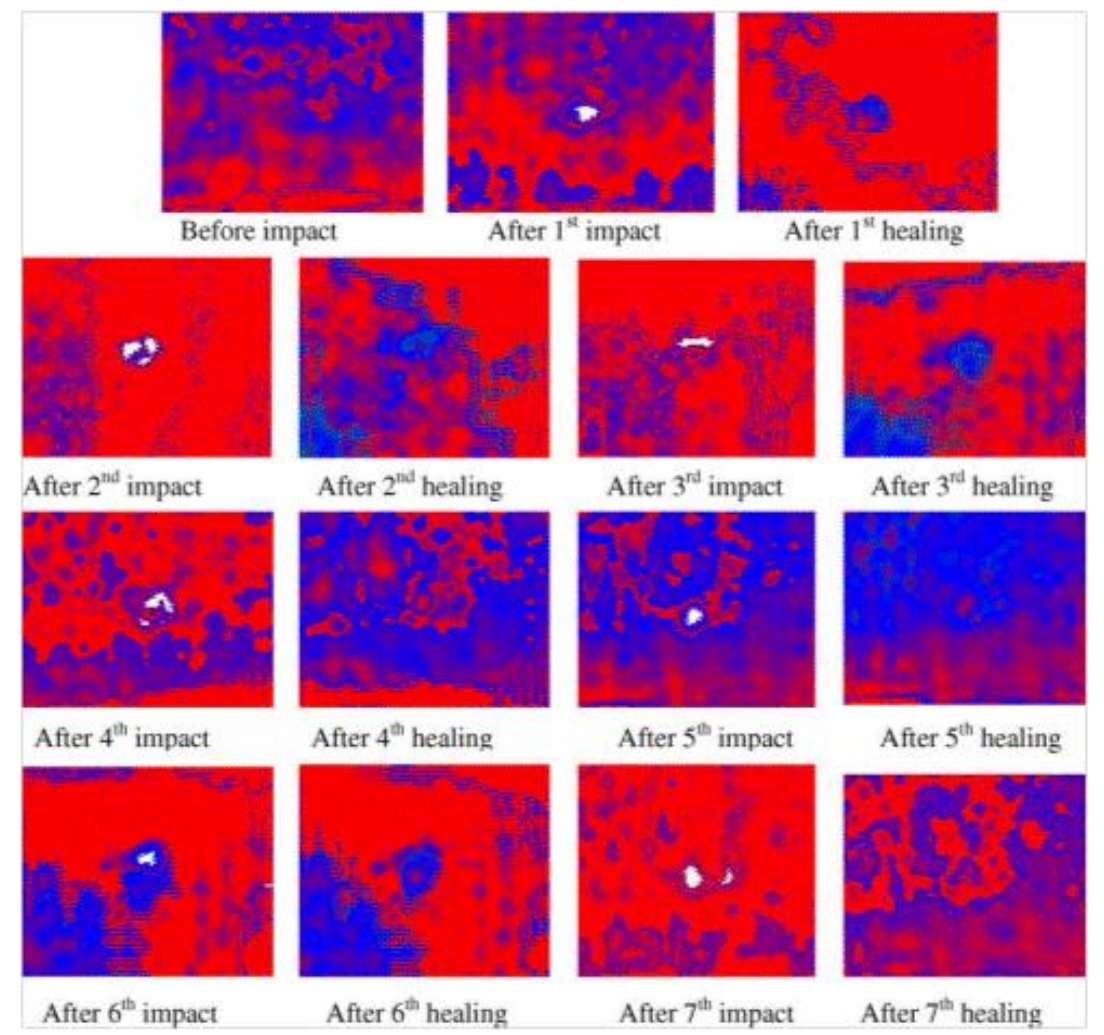

Figure 5: C-scan images of the sandwich panels after each impact and healing cycle. The figure is reproduced with permission from [47]. C-scan images give a visual representation of the attenuation of the ultrasound signal through the panel, which is proportional to the damage present, with $20 \%$ attenuation indicated by red color, $20-50 \%$ attenuation by blue color and $100 \%$ attenuation by white color. As the figure shows, even after the $7^{\text {th }}$ impact, the macro-length scale damage can be adequately healed by heating the foam above its $T_{g}$.

While this technology works well, problems arise in this application when the thermal expansion of the concrete walls takes place under higher ambient temperatures leading to development of compressive stresses in the foam core and cause it to 
squeezing out of the channel. An alternative 2D programming method of SMP foams was proposed to alleviate this issue. With this programming, the material could show "auxetic" properties leading to shrinkage of the material dimension in the longitudinal direction when a compressive stress is applied by thermal expansion of concrete walls in the lateral direction, above its transition temperature [71].

\section{Textiles}

In the textile industry, SMP foams have been investigated as intelligent and weather adaptive clothing materials. Chung et al. studied an open cell polyurethane based SMP foam for this purpose [27]. These SMP foams demonstrated good shape fixity at $20^{\circ} \mathrm{C}$, partial recovery at $30^{\circ} \mathrm{C}$, and full recovery within $2-3$ minutes $40{ }^{\circ} \mathrm{C}$ onwards. While the permeability and insulation of the foam was similar across this temperature range, the water vapor transmission rates were up to three times higher in the recovered state at $40{ }^{\circ} \mathrm{C}$. It was inferred that at standard ambient temperatures (20 ${ }^{\circ} \mathrm{C}$ ), the foam would retain its thin compressed state providing comfortable wear. As the environmental temperatures increase, however, it would expand with concomitant increase in the water vapor transmission rates for improved comfort.

\section{Sand Management}

SMP foams have been applied as tools for sand management, in the field of petroleum engineering. The metal screens, which have been traditionally used for this purpose, have the limitation of low conformity with the well bore surface, in addition to 
being costly and complicated [72]. As a substitute, annular SMP foam cylinders ( 6 feet long) with outer diameters larger than the well bore, and inner diameter adequate for the placement of base pipes have been used. The foam is initially compressed in the radial direction for installation in the well bore. On actuation, the foam expands and conforms to the bore wall. The force exerted thereby holds the sand in place and assists in maintaining an open well bore [73]. Tests performed by Carrejo and coworkers [74, 75] showed that SMP foam can adequately filter sand without compromising well productivity. Yuan and coworkers [72] further defined the working conditions under which SMP foam would be beneficial over the traditional gravel packing.

\section{Use of SMP Foams for Treatment of Intracranial Aneurysms}

Amongst the numerous applications of SMP foams discussed in the previous section, their use in the embolic treatment of intracranial aneurysms, is of particular interest to us $[6,8]$. This section aims to introduce this medical condition to the readers, starting with a brief overview of the pathology of intracranial aneurysms, and the currently used treatment methods. This is followed by the proposed mechanism of treatment of aneurysms using SMPs, and the limitations of the current commercially available SMPs, which form the basis of need and the motivation of this dissertation.

\section{Intracranial Aneurysms}

Aneurysm is a pathological condition in which an area of the blood vessel undergoes abnormal localized dilation or ballooning. Due to histopathologic and 
hemodynamic factors, these are most commonly found in arteries that supply blood to the brain. Autopsy and imaging studies report high frequencies of aneurysm detection $(1 \%$ to $9 \%[76,77]$ and $0.5 \%$ to $2 \%,[78])$, and the prevalence of intracranial aneurysms is estimated to be 83.4 per 100,000 people [79] . On the basis of this data, it is likely that at least 3 to 6 million people in the United States harbor an intracranial aneurysm [79].

A common feature of intracranial aneurysms is a significantly fragmented elastic lamina and muscular media $[80,81]$. Their exact cause is still a subject of debate. But broadly, the lack of cerebral elastic lamina and supporting perivascular tissue, sparse medial elastin, and structural irregularities at the apex of bifurcations are considered to be primary factors making cerebral arteries susceptible to the development of aneurysms [82, 83]. Genetic factors and habits like cigarette smoking, alcohol consumption, long term analgesics and oral contraceptive may also have a role in their development [84, 85]. If left untreated, these aneurysms continue to grow and can eventually rupture causing a hemorrhage (Figure 6).

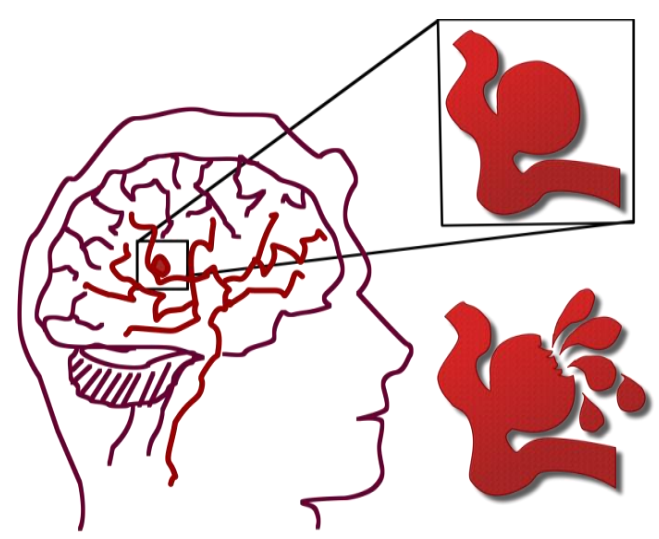

Figure 6: Rupture of an intracranial aneurysm may cause hemorrhage, and it is often fatal to the patient. 
Approximately 25,000-30,000 people in United States suffer from death or severe debilitation as a result of Sub-Arachnoid Hemorrhage (SAH) from aneurysm rupture [86]. An analysis of the physical factors influencing the rupture of an aneurysm showed that the probability of rupture increases with an increase in intra-aneurysmal pressure, an increase in aneurismal size, a decrease in the minimum wall thickness of an aneurysm, or a decrease in the strength of its structural components [78]. Once a rupture occurs, the statistics of post-hemorrhagic survival of the patient are quite grim: Nearly $10-15 \%$ (and for some studies as many as 30\%) of aneurismal SAH patients die before receiving adequate medical help $[87,88]$. Amongst the people who are able to receive timely medical care, about $30 \%-60 \%$ suffer death from the hemorrhage or related causes [89]. Mortality rate of patients within 30 days of the aneurysm rupture is $~ 50 \%$ with majority of these occurring within the initial fourteen days [87-89]. Further, surviving patients often suffer from serious neurological disorders such as paralysis, cranial nerve palsies, cognitive impairments, aphasia, psychiatric disturbances, and behavioral disorders [90]. Given the large prevalence of aneurysms, and the danger that they pose to patient's health and survival, it is important to develop safe and effective treatment procedures for this condition.

\section{Current Treatments of Intracranial Aneurysms}

\subsection{Surgical Clipping}

One of the popular current techniques of aneurysm treatment is surgical clipping, 
first used in 1937 by Dr. Walter Dandy. [91, 92] In this procedure, the patient is given anesthesia and a craniotomy is performed. The aneurysm is located through the opening made in the skull, and a small elgiloy or titanium based clip is placed across the neck of the aneurysm to block the flow of blood inside the aneurismal bulge (Figure 7). Aneurysms treated by this method have been observed to have a low rate of recurrence after treatment [91]. Recently a team of neurosurgeons from University of North Carolina have pioneered a new approach of endoscopic endonasal clipping for aneurysm treatment [93]. Via this procedure, aneurysms located near the skull base can be reached via the nasal cavity. As access through the nose is less invasive than the traditional craniotomy, this advancement is expected to be very significant for patients harboring aneurysms near the base of the skull [93].

Nonetheless, this method is invasive and is often not preferred in patients of advanced age, or unstable health. Also, the full surgery implies that the costs of the treatment and patient care are high. Finally, it entails a permanent metal implant in the brain, lasting the entire lifetime of the patient.

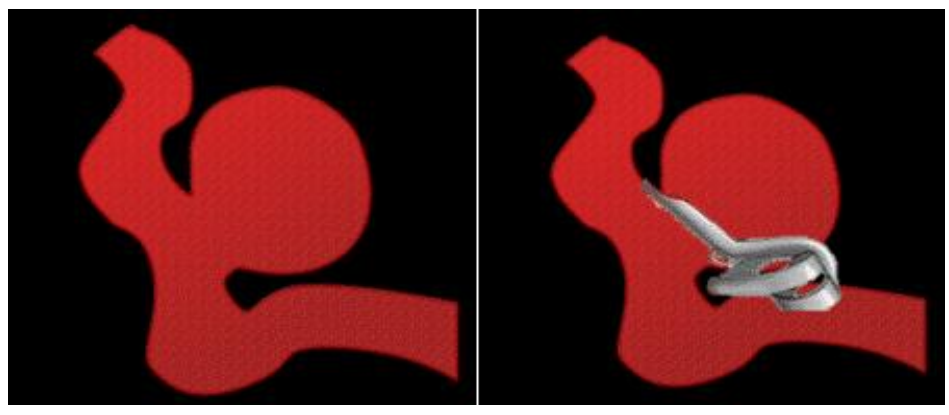

Figure 7: Surgical clipping: A technique for treatment of aneurysms where a metal clip is surgically placed at the aneurysm neck to avoid blood flow into it. 


\subsection{Guglielmi Detachable Coils}

Another popular technique used for treatment of aneurysms is using Guglielmi Detachable Coils (GDC). This technique is named after Dr. Guido Guglielmi, who established this detachable coil system, using a combination of electrothrombosis and endovascular techniques in the early 1990s [92]. It uses a catheterization process first described by Luessenhop and Velasquez in 1964 [92], and builds on the work of Serbinenko, often credited with the title of founder of endovascular surgery, who reported multiple catheters mounted with detachable balloons for intravascular occlusion applications in 1974 [92].

In this procedure thin platinum based coils are delivered to the aneurysm site through a catheter (Figure 8). First a microcatheter is placed in the aneurismal bulge, and a soft platinum based GDC coil soldered to a stainless steel delivery wire is delivered to the aneurysm site through the microcatheter. Once the coil is placed in a desirable configuration, a small $\sim 1 \mathrm{~mA}$ current is applied to the delivery wire. The current dissolves the stainless steel delivery wire proximal to the platinum coil by means of electrolysis, leaving the coil in place. Simultaneously, the positively charged platinum is thought to inducing intra-aneurismal thrombosis by attracting the negatively charged blood elements such as fibrinogen, platelets and white and red blood cells. Another coil is subsequently delivered to the aneurysm and the process is continued until the aneurysm is densely packed with the platinum coils, and no longer opacifies during the injections of diagnostic contrast agents. Variations of endovascular coiling techniques, such as stent assisted endovascular coiling have also been reported [92]. 


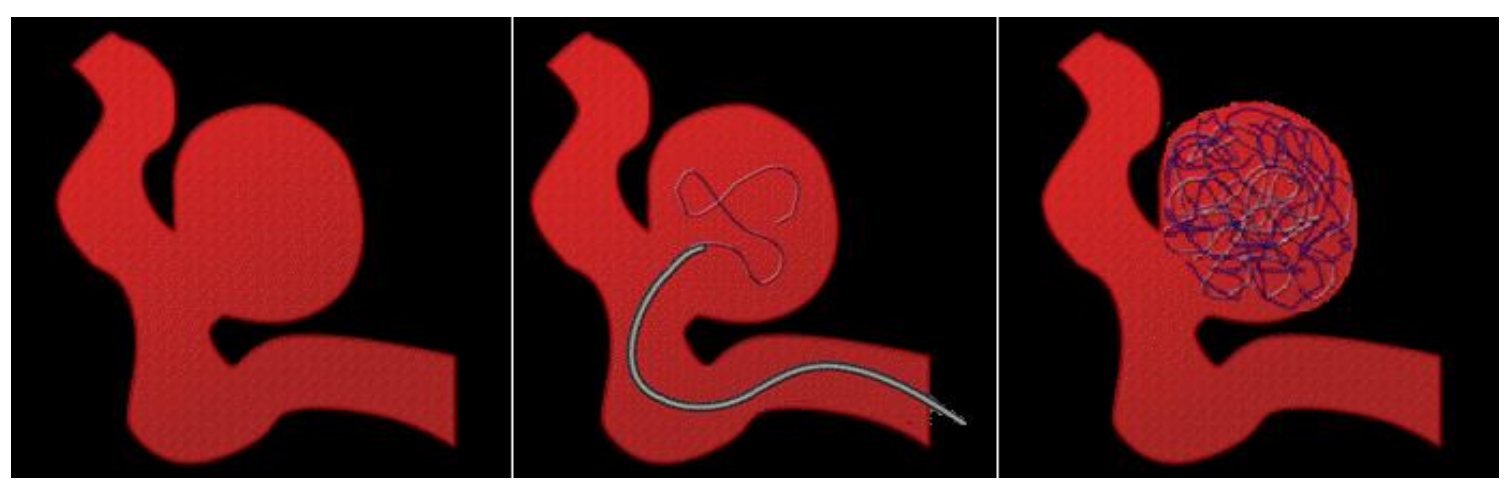

Figure 8: Treatment of aneurysm using Guglielmi detachable coils.

This technique, however, is successful in completely occluding only $\sim 85 \%$ of small necked aneurysms and $\sim 15 \%$ of large necked aneurysms [94]. In addition, there are other drawbacks. For instance, the deployment of coils is itself a long process in which more than one coils need to be placed inside the aneurysm depending on its size. Based on the technique used for the coil delivery, there is $1.4-2.7 \%$ chance of rupture of the aneurysm with every coil that is placed [86]. Other clinically proven drawbacks include incomplete occlusion of the aneurysm [95, 96], overtime coil compaction [97], shifting or even migration out of the aneurysm causing risks of aneurysm regrowth, rupture or stroke [98]. Further, like the clipping procedure, coiling also introduces permanent coil implants in the brain which are meant to stay for the lifetime of the patient. 


\subsection{Clipping vs. Coiling}

The treatment decision for surgical clipping, or endovascular coiling depends multiple factors such as the age of the patient, aneurysm's size, geometry and location, as well as the surgeon's personal preference/experience [92]. Due to its minimally invasive nature, endovascular coiling is preferred for patients of older age or serious/unstable health condition. Also, aneurysms at the basilar apex are favored for endovascular coiling, while the ones in the middle cerebral artery often have a complex geometry and are preferred to be treated by clipping/open surgery. Further, in some complicated conditions a combination of endovascular and surgical procedures may be adopted [92]. The comparative efficacy of coiling and endovascular techniques was studied by International Subarachnoid Aneurysm Trial (ISAT), the only large scale study of its kind. This study suggested that, for certain ruptured aneurysms, endovascular treatment may be an effective alternative to surgical treatment. However, the data was limited to some ruptured aneurysms and the results could not be generalized to claim definitive superiority of one or the other technique for all ruptured/unruptured aneurysms [92].

\section{Shape Memory Polymer Foams for Aneurysm Treatment}

The shape memory behavior renders SMP foams very useful for their potential application in the treatment of intracranial aneurysms $[6,8,99,100]$. By virtue of this property, these materials can be crimped to a small size $(<\sim 1 \mathrm{~mm})$ that can be placed inside a catheter. This crimped device can be delivered to the aneurysm site via the 
catheterization process through the femoral artery. Once at the target site, the device can be actuated by thermal stimulus to recover its primary shape, leading to occlusion of the aneurysm or limiting active blood flow to it (Figure 9). Subsequent formation of blood clot, recruitment of inflammatory cells, and later endothelialization of the aneurysm neck are expected to ultimately isolate the aneurysm, and eliminate the possibility of its rupture in the future.

The minimally invasive transcatheter procedures enabled by these materials can be significantly lower in cost and complications compared to the traditional open surgery. The high embolic efficiency of this device, lower modulus relative to metal coils, ability to have customized actuation temperature based on physiological requirements, ability to be biodegradable, and the excellent biocompatibility of SMPs in general, further enhance the potential biomedical utility of the SMP foams [6]. Hence SMP foams form an attractive alternative to the GDC procedure for aneurysm treatment.

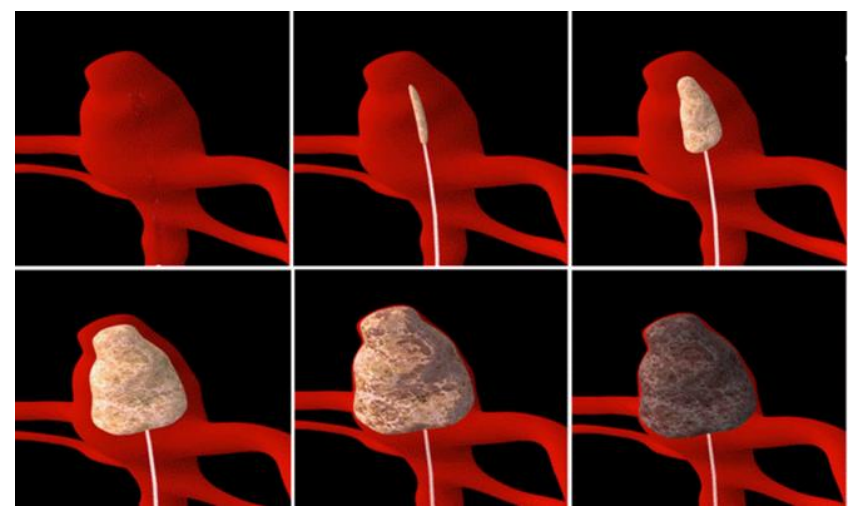

Figure 9: Schematic showing treatment of aneurysms, progressing from top left depicting an untreated aneurysm, to bottom right depicting an implanted foam device (Image Courtesy: Jennifer N. Rodriguez). 


\section{Limitations of Currently Available SMP Foams for this Application}

Despite the strong potential of SMP foams in the biomedical field there is limited availability of materials geared towards this application. Most of the shape memory foams reported for biomedical applications in the literature are based on a thermoplastic polyurethane developed by Mitsubishi Heavy Industries, primarily MF5520, MF6020 and MF21 [20-22]. Papers on characterization of Mitsubishi polymers themselves [21, $22,101-106]$, and other shape memory foams $[24,44,45,52]$ can be found. While these materials hold promise in several applications [7], some potential limitations from the perspective of catheter based biomedical applications were revealed in their characterization studies. In particular, the so-called "secondary-shape forming" was noticed in which irrecoverable deformation occurred when the material was stored under compression above the glass transition temperature $\left(T_{g}\right)$ [21]. In other words, depending on the storage conditions (strain and temperature), the foams could partially or fully lose their capability to expand. Also, their relatively high densities provide relatively low volume expansion of 20 - 30 times [20, 21]. For a foam cylinder that does not change

length, for example, this correlates to a maximum radial expansion of $\sim 5.5$ times $(=\sqrt{ } 30)$. For aneurysm occlusion via microcatheter delivery in which the inner working diameter is $\sim 0.5 \mathrm{~mm}$, this would give a final diameter of $2.75 \mathrm{~mm}$. This level of expansion may not be adequate for the proposed application: the greater the radial expansion of foam, the greater the volume that can be embolized by it for a given microcatheter lumen diameter. 


\section{Summary of the Dissertation}

The aim of this work was to develop a new class of materials specifically tailored towards the embolic biomedical application of treatment of intracranial aneurysms. This dissertation, which will focus on the design, formulation, fabrication, processing, and application specific characterization of these novel materials is organized as follows:

Chapter II discusses the design rationale of this new class of materials based on the desired set of physico-mechanical properties. In this chapter, the details of fabrication of these highly covalently crosslinked ultra low density foams, based on the hypothesis and optimized gas blowing technique initially developed by Dr. Thomas S. Wilson at LLNL, are presented. The results of characterization of foams are discussed for a better understanding of their chemical structure and key physical properties. Further, a comparison with other commercially available materials is done to exemplify the significance and utility of these shape memory foams as a biomaterial.

Chapter III builds on the polymer architecture proposed in Chapter II to introduce modification in chemical composition for achieving a controlled rate of actuation of the foams in physiological media/water. The strong hydrogen bonding potential of these materials was observed to cause a rapid depression in their actuation temperature on exposure to fluid hydrophilic media, leading to a pre-mature actuation. A controlled increment in composition hydrophobicity was implemented to overcome this problem. Chapter III discusses the formulation, synthesis, fabrication and characterization of these materials. Results of theoretical and experimental analysis are presented to illustrate that the hydrophobicity of the materials could be precisely 
controlled by changes in composition; and that this change is responsible for the predictable variation in the rate of actuation of the device in hydrophilic fluid media.

Chapter IV presents the methods and strategies employed for further reducing the density of the foams, and using plasma treatment for their complete reticulation. This signifies routes for important developments in the physical and morphological characteristics of the proposed device. Consequently, a higher embolic efficiency of the foam, as well as control over the rate of permeation of blood/physiological fluid during implantation can be achieved.

Finally, Chapter $\mathrm{V}$ further builds on the proposed polymer architecture to introduce hydrolysable linkages in the network chains. A biodegradable implant can thereby be achieved, and long term presence of a foreign material in the human body can be avoided. This chapter discusses the rationale of the study design and the method of foam fabrication. Further, results of rate of degradation over a period of 7 months, and the trends of the degradation rate and mechanism are presented. 


\section{CHAPTER II}

\section{ULTRA LOW DENSITY AND HIGHLY COVALENTLY CROSSLINKED SHAPE MEMORY POLYURETHANE FOAMS*}

Chapter I discussed the condition of intracranial aneurysms, and the limitations of currently available SMP foams for use in its treatment. Consequently, our aim in this study was to design a polymeric foam system customized for the embolic biomedical applications that can overcome the drawbacks of current materials. Particularly, we sought a material with strong shape memory behavior without secondary-shape forming, and a volume expansion of greater than 50 times on actuation. In addition, we aimed to achieve high recovery forces, controllable actuation temperatures, and good biocompatibility for these new materials.

The material design rationale was initially developed by Dr. Thomas S. Wilson at LLNL, and broadly two main points form its core. First, secondary-shape forming in principle occurs from the relaxation of polymer chains in the secondary or deformed shape [107-109]. So, a covalently crosslinked structure with high density of crosslinks can potentially avoid the secondary-shape forming phenomenon by providing a permanent and strongly constrained polymer network that limits chain relaxation [107109]. Second, for achieving a larger expansion ratio, lower density foam is desired. Low density can potentially allow a foam sample to be compressed to smaller dimensions and thus lead to a larger volume expansion on actuation. In view of these considerations,

\footnotetext{
*Parts of this chapter are adapted or reprinted with permission from Singhal P. et al. Ultra low density and highly crosslinked biocompatible shape memory polyurethane foams. J. Polym. Sci. B: Polym. Phys. 2012; 50 (10): $724-37$. Copyright 2012 Wiley Periodicals, Inc., A Wiley Company.
} 
specific choices of materials and foaming process were made in the foam development. Neat polymers reported by Wilson et al. [110] were used as the basis of the foam material. These neat polymers were made from $\mathrm{N}, \mathrm{N}, \mathrm{N}^{\prime}, \mathrm{N}^{\prime}$-tetrakis (2-hydroxypropyl) ethylenediamine (HPED), 2,2',2"-Nitrilotriethanol (TEA), and 1, 6 Diisocyanatohexane (HDI) [in addition to other monomers such as 1,3 Butanediol, TMHDI, IPDI)], and had a regular and highly covalently crosslinked network structure with low molecular weight between crosslinks [110] (Figure 10). They were developed specifically to possess high modulus $\left(E \sim 3 n_{c} R T\right.$, where $E$ is the Young's modulus, $n_{c}$ the number crosslinks per unit volume, $R$ the ideal gas constant and $T$ the system temperature), high recovery stresses, sharp and controllable actuation temperatures, and strong shape memory behavior [110]. The density of foamed polymer has a strong effect on its modulus; $E_{\text {porous }}=E_{\text {neat }}\left[\rho_{\text {porous }} d\right.$ $\left.\rho_{\text {neat }}\right]^{2}$ for open cell foams, where $E_{\text {porous }}$ and $E_{\text {neat }}$ are the Young's moduli of porous and neat materials and $\rho_{\text {porous }}$ and $\rho_{\text {neat }}$ are the densities of porous and neat materials respectively [111-113]. Hence these materials, with a neat glassy modulus in the GPa range, were expected to perform well at low densities. Their tight network structure with high density of covalent crosslinks was expected to limit secondary-shape forming. Also, the aliphatic monomers in this system were favorable for biocompatibility, as was demonstrated in an in vitro study [114]. Figure 11 and Table 2 give a schematic representation of the network structure, and the foam design rationale respectively. 
<smiles>CC(O)CN(CCN(CC(C)O)CC(C)O)CC(C)O</smiles><smiles>OCCN(CCO)CCO</smiles><smiles>O=C=NCCCCCCN=C=O</smiles>

$\mathrm{N}, \mathrm{N}, \mathrm{N}^{\prime}, \mathrm{N}^{\prime}$ Tetrakis (2-hydroxypropyl)

ethylenediamine (HPED)

2, 2', 2" Nitrilotriethanol (TEA)

1, 6 Diisocyanato hexane (HDI)

Figure 10: Molecular structures of the low molecular weight, symmetrical and polyfunctional monomers (HPED, TEA and HDI) used in the SMP foam synthesis.

a)

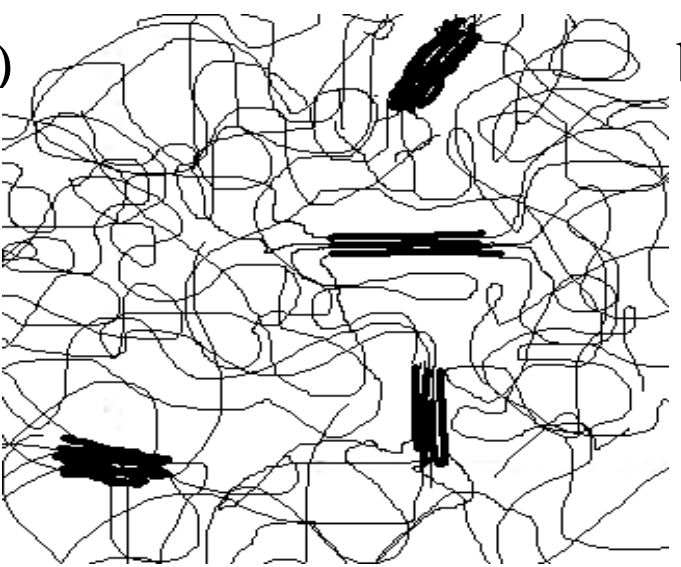

b)

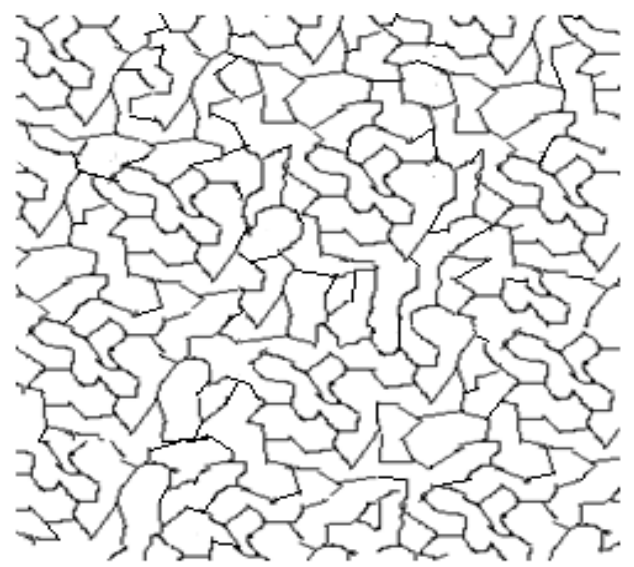

Figure 11: A schematic representation of the network structure of (a) physically crosslinked traditional polyurethane foams and (b) proposed covalently crosslinked foams from low molecular weight monomers. This figure is reproduced with permission from [30]. 
Table 2: Summary of the device requirements and design strategy adopted in the synthesis of these materials.

\begin{tabular}{|c|c|c|}
\hline $\begin{array}{l}\text { Device } \\
\text { requirements }\end{array}$ & Material characteristics & Device design strategy \\
\hline Long shelf life of device & $\begin{array}{l}\text { No secondary-shape forming, strong } \\
\text { shape fixity }\end{array}$ & Covalently crosslinked network structure \\
\hline $\begin{array}{l}\text { Ability to actuate at a } \\
\text { desired physiologically } \\
\text { compatible temperature }\end{array}$ & $\begin{array}{l}\text { Control over the actuation } \\
\text { temperature/glass transitions of the } \\
\text { foams }\end{array}$ & $\begin{array}{l}\text { Variation in the ratio of constituent monomers to vary } \\
\text { steric hindrance around crosslinks and crosslink } \\
\text { density }\end{array}$ \\
\hline $\begin{array}{l}\text { High volume expansion } \\
\text { from a single device }\end{array}$ & $\begin{array}{l}\text { Very low density foams and strong } \\
\text { shape recovery for complete return to } \\
\text { original shape }\end{array}$ & $\begin{array}{l}\text { A combination of chemical and physical blowing } \\
\text { techniques for low density } \\
\text { High crosslink density for good shape recovery }\end{array}$ \\
\hline $\begin{array}{l}\text { Ability to expand against } \\
\text { physiological media }\end{array}$ & $\begin{array}{l}\text { Good recovery forces i.e. high } \\
\text { modulus of foams even at very low } \\
\text { densities. }\end{array}$ & $\begin{array}{l}\text { As density has strong effect on resulting modulus } \\
\text { (Eporous= Eneat ( }(\rho \text { porous/ } / \text { neat)2), use neat polymer } \\
\text { with high modulus. } \\
\text { As } E \sim 3 n c R T \text {, have low molecular weight between } \\
\text { crosslinks for high } n c \text { i.e. employ low molecular } \\
\text { weight polyfunctional monomers in polymer network } \\
\text { formation }\end{array}$ \\
\hline $\begin{array}{l}\text { Free permeation of } \\
\text { blood/media through the } \\
\text { device as it expands }\end{array}$ & Open cell structure of the foams & $\begin{array}{l}\text { Choice of foaming additives favoring open cell } \\
\text { morphology }\end{array}$ \\
\hline Biocompatibility & Use of biocompatible monomers & $\begin{array}{l}\text { Use of aliphatic monomers and polyurethane } \\
\text { chemistry }\end{array}$ \\
\hline
\end{tabular}

For the fabrication of low density foams from the highly crosslinked neat materials, a gas blowing process based on a protocol initially developed by Dr. Thomas S. Wilson at LLNL was used. Multiple techniques are available for generation of a porous polymer structure, including particulate leaching, fiber bonding, saturation with supercritical gases, high internal phase emulsion polymerization, thermally induced phase separation, stereolithography, selective laser sintering, fused deposition modeling and gas foaming [115-122]. However, most of these methods, including particulate leaching [123], stereolithography [116], selective laser sintering and fused deposition modeling, do not 
yield low enough densities. Thermally induced phase separation is not suitable for crosslinked systems due to the requirement of a polymer solution. High internal phase emulsion polymerization is used primarily for chain growth reactions [124], or has low control of foam structure in step growth reaction [125]. Also, use of supercritical $\mathrm{CO}_{2}$ in foaming has poor control of foam density as the polymer crosslink density is increased [126]. Amongst these methods, gas foaming was considered to be the most promising technique for making low density foams based on polyurethane chemistry [127-129]. This technique was found to have no direct limitations on foaming of highly crosslinked materials. Also, multiple additives are available commercially for the gas blowing process, providing a large working space to modulate the physical properties of the foams.

\section{A. Materials and Methods}

\section{Foam Synthesis}

HPED (99\%, Sigma Aldrich Inc.), TEA (98\%, Alfa Aesar Inc.), HDI (TCI America Inc.), and DI water (Millipore water purifier system, Millipore Inc., $>17 \mathrm{M} \Omega \cdot \mathrm{cm}$ purity), were used as received. Foams were synthesized in a three step method. First a NCO premix, or prepolymer with excess isocyanate, was made by mixing 35-40 equivalents of hydroxyl groups from varying ratios of HPED and TEA, with every 100 equivalents of isocyanate groups. This NCO premix was allowed to cure for 2 days under nitrogen. Secondly, a hydroxyl $(\mathrm{OH})$ premix was made by mixing 
together all the balance hydroxyl groups with the surfactants and catalysts as per the amounts indicated in Table 3. Finally in the third step, the NCO premix and $\mathrm{OH}$ premix were mixed together in stoichiometric amounts, maintaining a 104 isocyanate index, along with the physical blowing agent, Enovate (HFC-245 fa). The mixture was kept in an oven at $90^{\circ} \mathrm{C}$, and was allowed to rise and subsequently cure for 20 minutes. The foam thus made was kept at room temperature for a week for additional cure, before any characterization studies.

Table 3: Foam formulation details as wt.\% of the final composition. This table is reproduced with permission from [30].

\begin{tabular}{|c|c|c|c|c|c|c|c|c|}
\hline $\begin{array}{c}\text { Sample } \\
\text { ID }\end{array}$ & $\begin{array}{c}\text { HDI } \\
\text { (wt. \%) }\end{array}$ & $\begin{array}{c}\text { HPED } \\
\text { (wt. \%) }\end{array}$ & $\begin{array}{c}\text { TEA } \\
\text { (wt. \%) }\end{array}$ & $\begin{array}{c}\text { Water } \\
\text { (wt.\%) }\end{array}$ & $\begin{array}{c}\text { Catalysts } \\
\text { (BL-22+T-131 at } \\
\mathbf{2 . 5 : 1} \text { mass ratio) } \\
\text { (wt. \%) }\end{array}$ & $\begin{array}{c}\text { Surfactants } \\
\text { (DC 4000 + } \\
\text { DC5169) } \\
\text { (wt. \%) }\end{array}$ & $\begin{array}{c}\text { Enovate } \\
\text { (pph) }\end{array}$ & $\begin{array}{c}\text { OH/NCO } \\
\text { ratio }\end{array}$ \\
\hline H80 & 61.8 & 23.6 & 4.0 & 2.7 & 0.9 & 7.0 & 7.4 & 0.96 \\
\hline H60 & 63.1 & 18.1 & 8.2 & 2.8 & 0.9 & 7.0 & 7.5 & 0.96 \\
\hline H40 & 64.4 & 12.3 & 12.6 & 2.8 & 0.9 & 7.0 & 7.5 & 0.96 \\
\hline H20 & 65.8 & 6.3 & 17.1 & 2.9 & 0.9 & 7.1 & 7.6 & 0.96 \\
\hline H0 & 66.7 & 0.0 & 22.2 & 3.0 & 0.4 & 7.7 & 15.4 & 0.96 \\
\hline
\end{tabular}

The wt.\% value represents the net amount of the component in the final foamed polymer.

A series of foams, with varying ratios of TEA vs. HPED in the net formulation, were prepared as indicated in Table 3 to control the polymer $T_{g}$. The notation H80, H60, H40, H20 and H0 denotes HPED equivalents satisfying 80, 60, 40, 20 and 0\% isocyanate equivalents, respectively, excluding the $41 \%$ equivalents satisfied by water. These correspond to the neat/unfoamed compositions of A3, A5, A7, A9 and A11, respectively, as reported by Wilson et al.[110]. As the final foam density is dependent on the concentration of gas present in the foaming solution at any given time [129], a 
combination of physical and chemical blowing processes was used in the foam synthesis to increase the effective concentration of gas in the foaming polymer. Also, to prevent formation of large voids in the foam in case the gas concentration goes beyond the Critical Limiting Supersaturation (CLS) level of the foaming solution [129], simultaneous adjustment of other foaming additives, such as surfactants and catalysts, was performed to control the rate of the polymerization reaction, formation of foam cells and drainage of polymer from the cell membranes [130]. A surfactant type was chosen through qualitative consideration of monomer and blowing agent cohesive energies involving their polarity, hydrogen bonding potential and dispersive forces. For controlling the rate of isocyanate reaction with water and other hydroxyl monomers, a two catalyst system was used, which included a) a general amine-based catalyst for $\mathrm{CO}_{2}$ generation and b) a tin-based catalyst for gelation reaction. Optimization of the net formulation was done empirically with a starting point based on the supplier's (Air Products and Honeywell Corp.) foam additive specifications, and the previous literature on traditional polyurethane foams [129]. Table 3 summarizes the synthesis details of these materials for all tested compositions.

\section{Foam Characterization}

\subsection{Density and Cell Structure}

Core density of a representative foam sample was measured from top, middle and bottom sections of the foams as per the ASTM standard D-3574-08. Also core 
density of five samples from the top and middle sections was measured for each foam formulation to estimate the variation in densities across a given foam. For cell structure characterization, thin slices were cut from a representative top section of the foams, and images were captured in the brightfield mode on a Leica MZ8 microscope (Leica Microsystems Inc.) using RSImage Software (Roper Scientific Inc.).

A more in-depth cell structure analysis was done on a H60 foam sample using micro-CT imaging. The sample was imaged using a Skyscan 1172 micro-CT [131] (Micro Photonics Inc.) with a $40 \mathrm{kV}$ source voltage, $250 \mu \mathrm{A}$ source current, object to source distance of $49.48 \mathrm{~mm}$ and camera to source distance of $208.96 \mathrm{~mm}$. These settings resulted in a reconstructed volume of foam that had a resolution of $4.15 \mu$ per voxel. For analysis of cell sizes in the reconstructed foam volume, image processing software Amira 5.3 (Visage Imaging Inc.) was used. Polar diameter (parallel to the direction of foam rise) and equatorial diameter (perpendicular to the direction of foam rise) of the ellipsoid-like cells were measured at $500 \mu$ increments throughout the volume via manual edge detection method. In all, a cylindrical volume $6 \mathrm{~mm}$ in diameter and $5.12 \mathrm{~mm}$ high was analyzed. Foamview software [131] was used to estimate the average strut length and pore size of the foams.

\subsection{Solvent Swelling and Extraction}

Solvent swelling and extraction experiments were carried out with dimethylformamide (DMF; >99.8\%, EMD Chemicals Inc.) as this was found to be the best solvent for such compositions [110]. Cylindrical foam samples ( $8 \mathrm{~mm}$ diameter and 
$2 \mathrm{~cm}$ height) were pre-dried for 2 hours at $100{ }^{\circ} \mathrm{C}$ and 1 atm vacuum. The dried samples were extracted in DMF at a 25 times excess of the bulk volume of foams. Care was taken to remove the bubbles on the foam surface that can prevent adequate contact of the material with solvent. The extraction was performed on three samples of each composition for 24 hours. Post extraction the samples were vacuum dried for 24 hours at $50{ }^{\circ} \mathrm{C}$ and mass loss was measured.

2.3 Fourier Transform Infrared Spectroscopy with Attenuated Total Reflectance (FTIRATR)

The FTIR-ATR spectra was generated on a H60 foam and the corresponding neat sample using a ATR Max II reflectance accessory (Pike Technologies Inc.) on a Tensor 27 Fourier Transform Infrared instrument (Bruker Corp.) at a 45 degree angle of incidence. 150 scans at a resolution of $4 \mathrm{~cm}^{-1}$ were taken in the wavenumber range of $4000 \mathrm{~cm}^{-1}$ to $600 \mathrm{~cm}^{-1}$ and background was subtracted. Testing was done in triplicate to ensure repeatability. Correction for atmospheric Water vapor and $\mathrm{CO}_{2}$ absorption was performed by subtracting respective reference spectra using the OPUS 5.5 software (Bruker Corp.).

\subsection{Differential Scanning Calorimetry (DSC)}

$T_{g}$ was measured using a Pyris Diamond DSC (Perkin Elmer Inc.). A 3-5 mg sample was loaded in a vented aluminum pan at room temperature, cooled to $-40{ }^{\circ} \mathrm{C}$ and then run through a heat - cool - heat cycle from -40 to $120{ }^{\circ} \mathrm{C}$. The half-height of 
transition during second heat was taken as an estimate of the $T_{g}$.

\subsection{Dynamic Mechanical Thermal Analysis (DMTA)}

For characterizing the mechanical properties of the foams as a function of temperature, a dynamic temperature ramp test was performed on ARES-LS2 rheometer (TA Instruments Inc.). A torsion rectangle test fixture was used on samples cut to approximately $45 \mathrm{~mm}$ long, $12 \mathrm{~mm}$ wide, and $6 \mathrm{~mm}$ thick with a gap distance of $25 \mathrm{~mm}$. The samples were prepared by embedding both ends in a polyurethane neat polymer to prevent damage and slippage of the foam sample in the metal grips. Dynamic temperature ramp tests were then run for each formulation in triplicate, at a frequency of

$1 \mathrm{~Hz}$ and constant heating rate of $1{ }^{\circ} \mathrm{C} \cdot \mathrm{min}^{-1}$ from 25 to $120^{\circ} \mathrm{C}$. An initial shear strain of $0.2 \%$ was used. However, as temperature increased, it was adjusted by the control software to maintain a torque range of 0.5 to $5 \mathrm{~g} \cdot \mathrm{cm}$, allowing a maximum strain of $10 \%$ (still within the linear viscoelastic region) at high temperatures. Data points were collected every 5 seconds. Dynamic shear storage modulus $\left(G^{\prime}\right)$, dynamic shear loss modulus $\left(G^{\prime \prime}\right)$, and their ratio $\tan \delta\left(=G^{\prime \prime} / G^{\prime}\right)$ were recorded using the Orchestrator ${ }^{\mathrm{TM}}$ software (TA Instruments Inc.).

\subsection{Shape Memory Behavior}

For measuring the shape memory behavior, constrained stress recovery tests were performed in compressive mode using parallel plate fixtures in a ARES-LS2 rheometer (TA Instruments, Inc.) on H60 and H20 cylindrical foam samples ( 20 mm diameter and 
$15 \mathrm{~mm}$ height). The sample was first heated up to a temperature of $T_{g}+30{ }^{\circ} \mathrm{C}$ and deformed to a $80 \%$ compressive strain at a rate of $2.5 \mathrm{~mm} \cdot \mathrm{min}^{-1}$. Thereafter the sample was cooled to $T_{g}-20{ }^{\circ} \mathrm{C}$ and then heated back up to $T_{g}+30{ }^{\circ} \mathrm{C}$ maintaining the $80 \%$ strain. At the end of cool-heat cycle, the strain was released at a rate of $2.5 \mathrm{~mm} \cdot \mathrm{min}^{-1}$ and recovered strain was measured from the distance between the plates at a $10 \mathrm{~g}$ axial force. Five cycles were performed on each of the three samples tested.

\subsection{Effect of Storage Temperature on Shape Recovery}

The behavior of stress vs. time was studied for H60 and H20 cylindrical samples (20 $\mathrm{mm}$ diameter and $20 \mathrm{~mm}$ height), using parallel plate fixtures in ARES-LS2 rheometer (TA Instruments, Inc.). Sample size and test conditions were chosen to match the test method reported by Tobushi et al. [21]. A deformation to $80 \%$ compressive strain was performed at a rate of $2.5 \mathrm{~mm} \cdot \mathrm{min}^{-1}$ at $T_{g}+30{ }^{\circ} \mathrm{C}$ temperature. The compressed sample was then brought to the test temperature, $T_{g}+60{ }^{\circ} \mathrm{C}, T_{g}+30{ }^{\circ} \mathrm{C}, T_{g}$ or $T_{g}-30{ }^{\circ} \mathrm{C}$, and held at this temperature for 2 hours. Thereafter the temperature was brought back to $T_{g}+30{ }^{\circ} \mathrm{C}$ and strain was released at $2.5 \mathrm{~mm} \cdot \mathrm{min}^{-1}$. The strain recovery was measured using calipers. All the compositions were tested for $\%$ shape recovery after hold at the $T_{g}+60{ }^{\circ} \mathrm{C}$ temperature.

\subsection{Maximum Volume Expansion}

Maximum volume expansion for foam samples was measured using a SC150-42 Stent Crimper (Machine Solutions Inc.). Cylindrical foam samples $6 \mathrm{~mm}$ in diameter 
were loaded in the crimper and compressed as small as possible, at $T_{g}+30{ }^{\circ} \mathrm{C}$, by setting the target diameter to zero. They were then allowed to cool down to room temperature to fix the compressed shape. The actual compressed diameter was measured using a digital micrometer. Finally, the samples were heated back to $T_{g}+30{ }^{\circ} \mathrm{C}$ in air, and the recovered diameter was measured using calipers. Net volume expansion was calculated (ignoring any change in length) as follows:

Volume expansion $=(\text { recovered diameter } / \text { compressed diameter })^{2} \quad($ Eqn. 2.1)

\subsection{Biocompatibility Study*}

In vitro biocompatibility testing was performed to estimate the biological response of these foams. Representative H80 foam samples were first cut into discs of approximately $12 \mathrm{~mm}$ diameter and $2 \mathrm{~mm}$ thickness, and then cleaned and sterilized using ethylene dioxide gas. Biocompatibility was evaluated by measuring cytokine expression as per Cabanlit et al. [114]. Blood of 5 female and 5 male human subjects was used following the approved human subject protocol 993120, University of California, Davis. Peripheral blood from these subjects was collected and stored in citrate tubes which allowed the stabilization of pertinent cell populations of the cell culture. Next, the collected blood samples were centrifuged at $3000 \mathrm{rpm}$ for 10 minutes and the supernatant plasma was separated. For testing the material biocompatibility, SMP foam discs were incubated using $X$-vivo media (serum-free medium; Cambrex Corp.) and 1:1 plasma-free whole blood. Positive controls included two mitogens, lipopolysaccharide (LPS, Sigma Aldrich Inc.) and phytohemagglutinin (PHA, Sigma

*The biocompatibility study was performed by Dr. Judy Van de Water and group at the campus of University of California, Davis, and their support for this work is gratefully acknowledged. 
Aldrich Inc.), added to the whole blood cultures at amounts of $25 \mu \mathrm{g} \cdot \mathrm{mL}^{-1}$ and 50 $\mu \mathrm{g} \cdot \mathrm{mL}^{-1}$ respectively. As a measure of the background activation, a control test of cells incubated in media without SMP foam material was performed. The samples were kept incubated at $37{ }^{\circ} \mathrm{C}$ in 24 -well plates for 48 hours. This duration was chosen to allow measurable production of all cytokines, including those derived from T lymphocytes.

After the 48 hour incubation, the culture supernatants were collected and stored at $-20{ }^{\circ} \mathrm{C}$. These were then tested with an enzyme-linked immunosorbent assay (ELISA, Duoset ELISA Development System kits (R\&D Systems Inc.)) for TNF- $\alpha$, IL-1 $\beta$, IL-6, IL-8, and IL-12 according to the manufacturer's instructions. Optical density readings at $570 \mathrm{~nm}$ were subtracted from those at $450 \mathrm{~nm}$ to account for optical imperfections. Mean optical density was recorded for TNF- $\alpha$, IL-1 $\beta$, IL-6, IL-8, and IL-12 over duplicate sample runs. A standard linear curve of optical density reading versus concentrations $\left(\mathrm{pg} \cdot \mathrm{mL}^{-1}\right)$ on a $\log -\log$ scale was used to convert optical density values to concentrations, and their average and standard deviations were calculated. P-values (one-tailed t-test) were calculated to determine if cell activation was significantly higher compared to the media alone (0.05 significance level).

\section{B. Results and Discussion}

\section{Physico-mechanical Properties}

The result of the physico-mechanical tests of SMP foams are summarized below. 


\subsection{Density and Cell Structure}

Table 3 shows the composition of different foams analyzed in this study, and representative densities of the foams are reported in Table 4. Average densities of $0.021 \pm 0.002,0.019 \pm 0.001,0.016 \pm 0.001,0.021 \pm 0.001$ and $0.020 \pm 0.005 \mathrm{~g} \cdot \mathrm{cm}^{-3}$ were recorded for foam compositions of $\mathrm{H} 0$ through $\mathrm{H} 80$ respectively over five samples in the top and middle sections. These correspond to an average porosity $\left[=\left(\rho_{\text {neat }}-\rho_{\text {porous }}\right) \cdot 100 / \rho_{\text {neat }}\right]$ of $\sim 98 \%$ and a high average theoretical volume expansibility $\left[=\rho_{\text {neat }} / \rho_{\text {porous }}\right]$ of $64 \pm 9$ times; here $\rho_{\text {neat }}$ is the neat polymer density, and $\rho_{\text {porous }}$ is the foam density. The small variation in densities in middle and top sections of the foam indicates a fairly uniform structure. Comparatively higher density is seen in the bottom section of foams in some cases. This is attributed to the effect of gravity on the rising foams as is typically seen in blown foams [132], and is accounted for in the foam characterization by taking the top/middle areas of the foams for the characterization tests.

Optical microscopy (Figure 12) shows a mixed closed to open cell structure for these foams, with thin residual membranes on the foam cells. Here the terms open or closed cell should be considered with reservations. As some texts classify foams into just two categories of closed and open cells [129], and others classify them into three categories of closed, open and reticulated cells [133], these terms can be ambiguous. Generally, blown foams have been found to retain thin residual membranes postsynthesis, and removal of these membranes (up to 97\%) to make foams completely open cell or reticulated invariably requires secondary physical processes such as hydrolysis, 
oxidation, heat or mechanical treatment [129]. Here, we classify these as-processed foams as effectively closed cell, or mixed closed to open cell, due to the presence of cell membranes which may impede the free movement of blood or interstitial fluid through the foam.

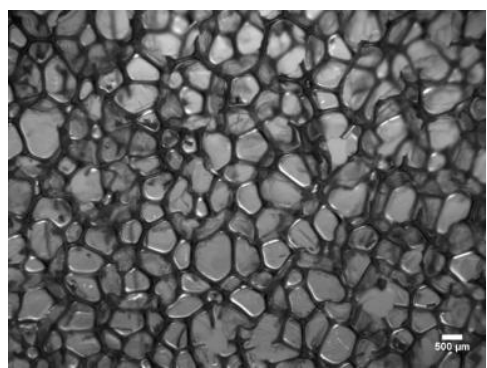

H80

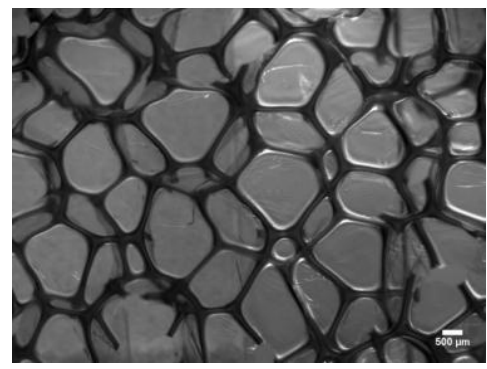

$\mathrm{H} 20$

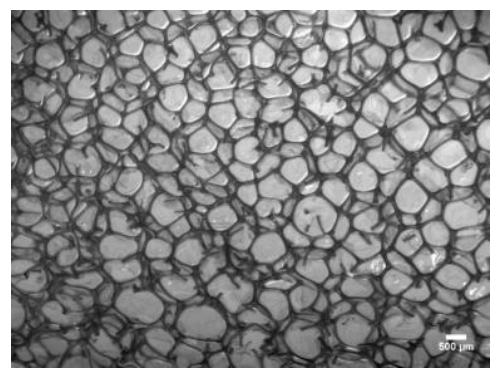

H60

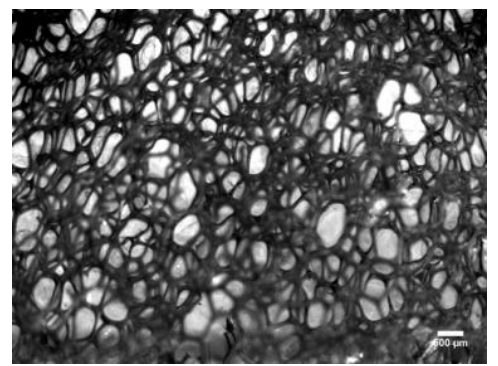

HO

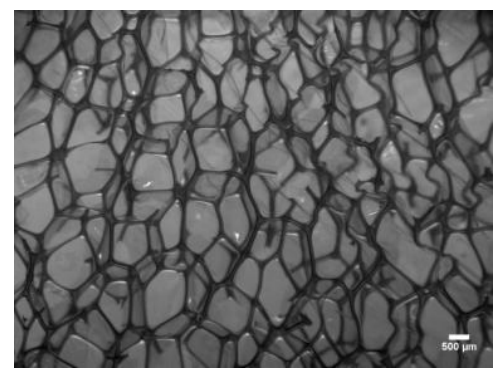

$\mathrm{H} 40$

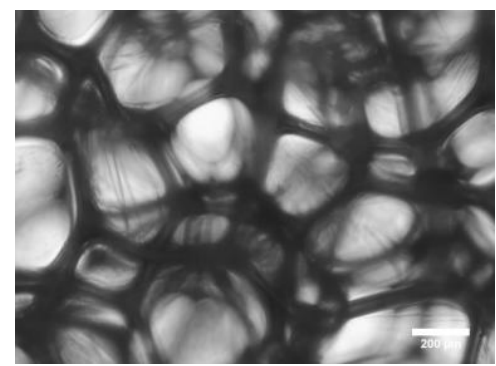

Magnified image of H60

Figure 12: Representative cell structure of foams for all the formulations $\mathbf{H 8 0}$ through Ho. Foams of all formulations show a mixed, closed to open cell structure, with thin cell membranes between struts. The residual thin cell membranes can be seen in the magnified image. All scale bars are $500 \mu$ except the magnified image where the scale bar is $200 \mu$. This figure is reproduced with permission from [30]. 
Table 4: Summary of key properties of the foams. This table is reproduced with permission from [30].

\begin{tabular}{|c|c|c|c|c|c|c|c|c|c|c|c|c|}
\hline Sample ID & $\begin{array}{c}\text { Density } \\
\text { Top } \\
\left(\mathrm{g} \cdot \mathrm{cm}^{-3}\right)\end{array}$ & $\begin{array}{l}\text { Density } \\
\text { Middle } \\
\left(\mathrm{g} \cdot \mathrm{cm}^{-3}\right)\end{array}$ & $\begin{array}{l}\text { Density } \\
\text { Bottom } \\
\left(\mathrm{g} \cdot \mathrm{cm}^{-3}\right)\end{array}$ & $\begin{array}{c}\text { Gel } \\
\text { fraction } \\
(\%)^{a}\end{array}$ & $\begin{array}{c}T_{g} \\
(\mathrm{DSC}) \\
\left({ }^{\circ} \mathrm{C}\right)\end{array}$ & $\begin{array}{c}G_{\text {glassy }}^{\prime} \\
\text { at } T_{\delta s^{-20}}{ }^{\circ} \mathrm{C} \\
(\mathrm{kPa})^{\mathrm{a}}\end{array}$ & $\begin{array}{c}G_{\text {rubbery }}^{\prime} \\
\text { at } T_{\delta s}+20^{\circ} \mathrm{C} \\
(\mathrm{kPa})^{\mathrm{a}}\end{array}$ & $\begin{array}{l}T_{o n s e t} \\
\left({ }^{\circ} \mathrm{C}\right)^{a}\end{array}$ & $\begin{array}{c}T_{\delta s} \\
\left({ }^{o} \mathrm{C}\right)^{\mathrm{a}}\end{array}$ & $\begin{array}{c}\Delta T \\
\left({ }^{\circ} \mathrm{C}\right)^{\mathrm{a}}\end{array}$ & $\begin{array}{c}\operatorname{Tan} \delta \\
{\text { (Peak value })^{\mathrm{a}}}^{2}\end{array}$ & $\begin{array}{c}\text { Volume } \\
\text { expansion }\end{array}$ \\
\hline H80 & 0.017 & 0.018 & 0.026 & $98 \pm 1$ & 69 & $223 \pm 18$ & $10 \pm 2$ & $64 \pm 2$ & $76 \pm 1$ & $24 \pm 6$ & $0.8 \pm 0.1$ & 71 \\
\hline H60 & 0.021 & 0.021 & 0.022 & $96 \pm 2$ & 61 & $255 \pm 49$ & $11 \pm 3$ & $55 \pm 1$ & $71 \pm 1$ & $31 \pm 1$ & $0.9 \pm 0.1$ & 68 \\
\hline H40 & 0.015 & 0.015 & 0.017 & $96 \pm 2$ & 57 & $212 \pm 63$ & $6 \pm 2$ & $49 \pm 1$ & $64 \pm 1$ & $31 \pm 7$ & $0.8 \pm 0.1$ & 71 \\
\hline H20 & 0.018 & 0.020 & - & $97 \pm 1$ & 47 & $235 \pm 25$ & $8 \pm 1$ & $40 \pm 0$ & $57 \pm 1$ & $32 \pm 3$ & $0.9 \pm 0$ & 68 \\
\hline Ho & 0.023 & 0.019 & 0.021 & - & 44 & $259 \pm 29$ & $11 \pm 1$ & $37 \pm 0$ & $52 \pm 1$ & $31 \pm 1$ & $0.7 \pm 0.1$ & 62 \\
\hline
\end{tabular}




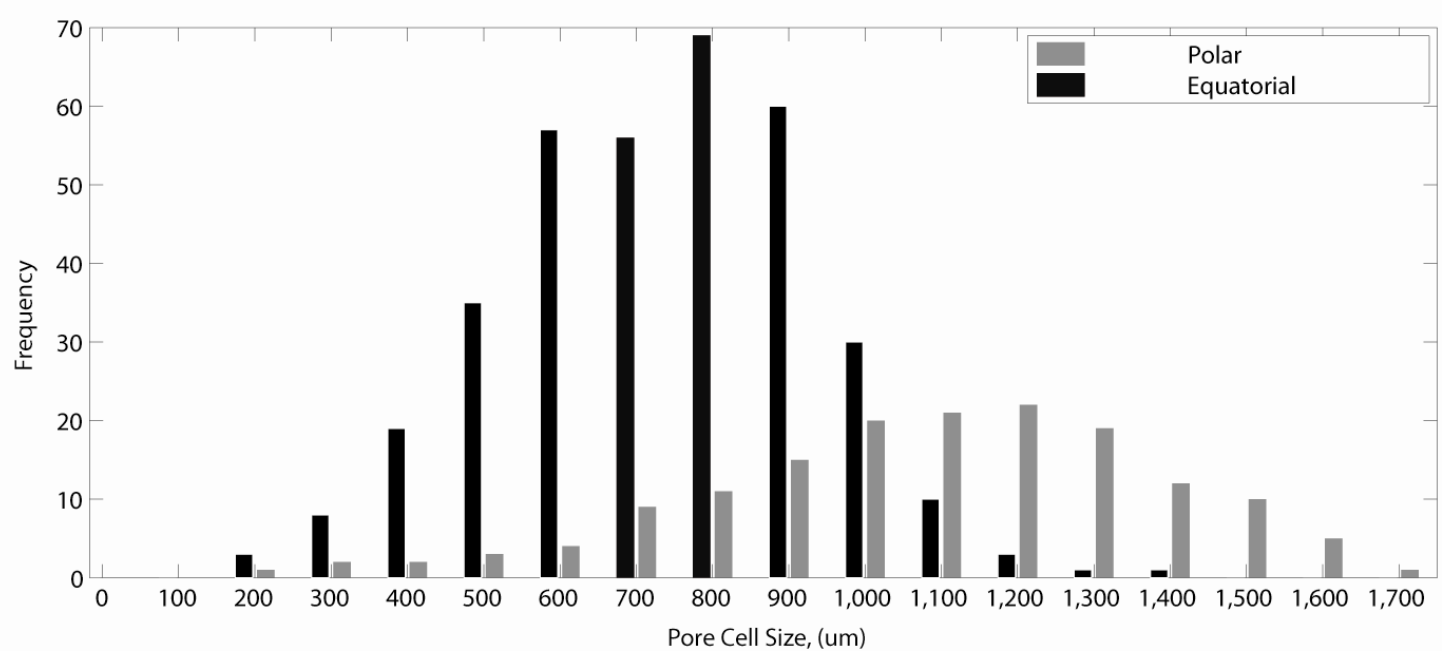

Figure 13: Histogram showing cell size distribution of H60 foam. It is seen that the pore cells are anisotropic, being taller, approximately $1000 \mu$, than they are wide, approximately $700 \mu$. Here equatorial measurements represent lateral diameters of pores, perpendicular to the direction of foaming; and the polar measurements represent the longitudinal diameters of pores parallel to the direction of foaming. This figure is reproduced with permission from [30].

Some variation in the average cell size was seen for foams across different formulations (Figure 12). This could be due to the different hydrophobicity, or different viscosity of the foaming solutions across the formulations at varying ratios of HPED and TEA. Indeed, in a separate study in which the viscosity of the foaming solution was the manipulated parameter, cell sizes of resulting foams could be precisely controlled, and were generally seen to decrease with increase in the viscosity of the foaming solution. Figure 13 shows a histogram of cell size distribution within a $\mathrm{H} 60$ foam sample. Its pore cells were found to be anisotropic as is typically seen for blown foams [129]. They were approximately $1000 \mu$ tall in the direction of free-rise during foaming, and $700 \mu$ wide. Using the image analysis software Foamview [131], the H60 foams were found to have 
strut length of approximately $400 \mu$, and an average pore volume of $7 \times 10^{8} \mu^{3}$.

Currently in the literature low density foams have been reported mostly down to the lower limit of $0.02-0.03 \mathrm{~g} \cdot \mathrm{cm}^{-3}$. Some foams with densities down to $0.006 \mathrm{~g} \cdot \mathrm{cm}^{-3}$ have been reported, but a high amount of water ( 75 wt. \%) was used in their synthesis [134]. This is not preferable for our application, as using high amounts of water as a chemical blowing agent will interfere with the desired covalently crosslinked network structure of the material. Also foams with densities down to $0.016 \mathrm{~g} \mathrm{~cm}^{-3}$ have been achieved by varying the polyol type and amounts [135]. However, to our knowledge, SMP foams with densities less than $0.02 \mathrm{~g} \cdot \mathrm{cm}^{-3}$ have not been previously reported. Also, low density SMP foams with a network structure involving such high density of chemical crosslinks have not been reported.

\subsection{Solvent Swelling and Extraction}

Solvent extraction results for all foam formulations are given in Table 4. The foam samples were all well swollen and without air bubbles in the DMF media. Results show that approximately 96 to $99 \%$ of the initial monomers were incorporated into the polymer network. The composition of the soluble fraction was not determined, but it is expected to be a combination of residual foaming additives, such as surfactants and catalysts, and impurities in the original monomers. Also, some mass loss was apparent from slight mechanical damage to the foams incurred during removal of bubbles. This indicates that $>85 \%$ of functional groups were consumed in reaction [110] and that the resulting network is indeed a highly crosslinked one. 


\subsection{FTIR-ATR}

FTIR-ATR of a H60 foam and corresponding neat polymer is shown in Figure 14. The neat polymer spectrum is offset by 0.03 absorbance units with respect to the foam spectra for clarity. Details of the peak assignment in polyurethane foams can be found in other references [136-139]. The absorption spectra showed a strong $\mathrm{C}=\mathrm{O}$ urethane peak at $1689 \mathrm{~cm}^{-1}$. A $\mathrm{C}=\mathrm{O}$ peak has previously been reported for polyurethanes at $1703-1710 \mathrm{~cm}^{-1}$ [138], $1694 \mathrm{~cm}^{-1}$ [139] and 1706-1713 $\mathrm{cm}^{-1}$ [136] for hydrogen bonded urethane, and 1730-1740 $\mathrm{cm}^{-1}$ [138], 1729-1739 $\mathrm{cm}^{-1}$ [136] and $1725 \mathrm{~cm}^{-1}$ [139]

for free urethane. Hence a shift of $\mathrm{C}=\mathrm{O}$ peak to $1689 \mathrm{~cm}^{-1}$ in these foams suggests a more strongly hydrogen bonded structure than is seen with previously reported polyurethane foams [136-139]. Based on the molecular structure of these foams, an average theoretical molecular weight between chemical crosslinks can be calculated as 270-440 g. $\mathrm{mol}^{-1}$. Further, approximately 2-3 urethane and/or urea bonds may be present between the chemical crosslinks for every segment. Hence these foams are expected to develop a high density of hydrogen bonds, which explains the shift of the urethane peak to a lower wavenumber in the FTIR spectra. This is in contrast with the traditional polyurethane foams, which typically have base polyols with molecular weights in the range of a few thousand grams per mole [129]. 


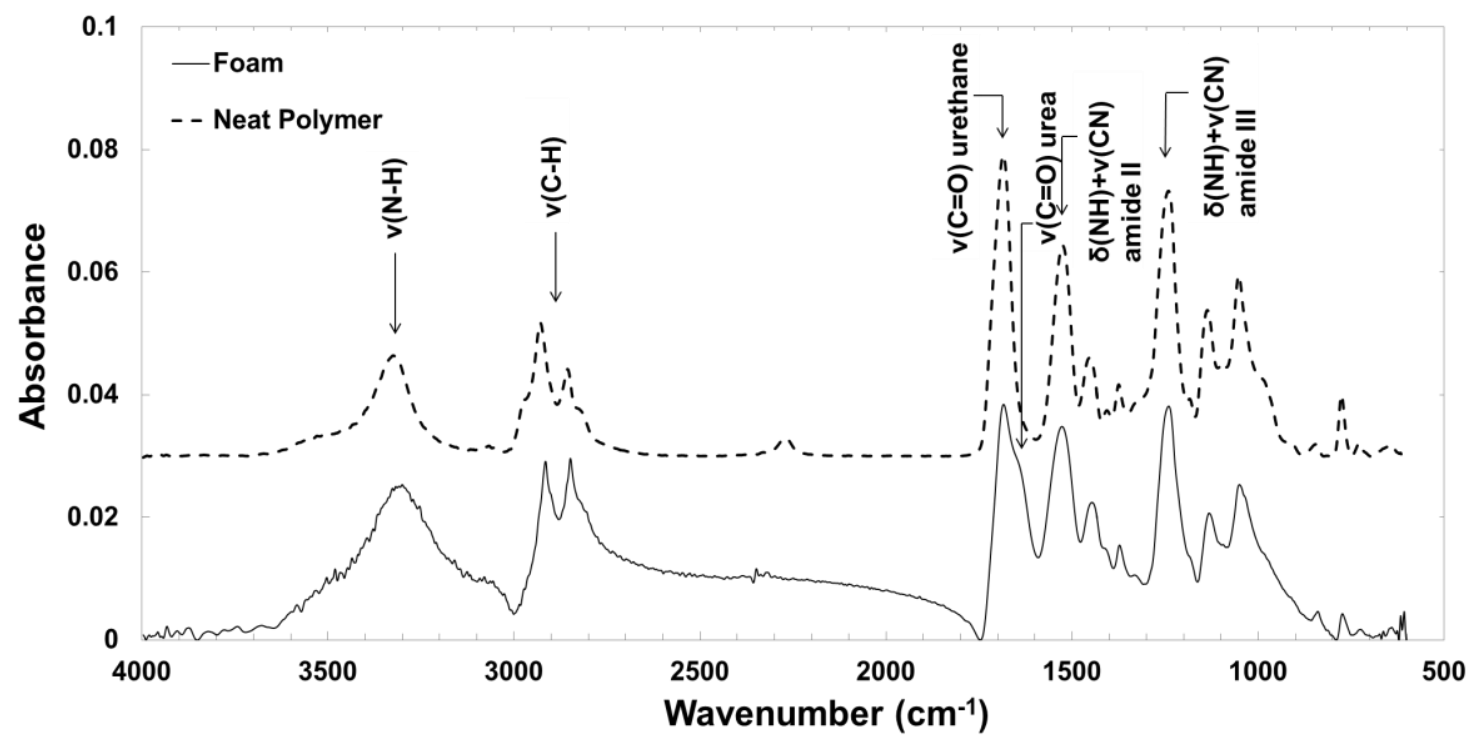

Figure 14: A comparison between the FTIR-ATR spectra of an unfoamed neat polymer (offset by 0.03 absorbance units) and foam of corresponding formulation. This figure is reproduced with permission from [30].
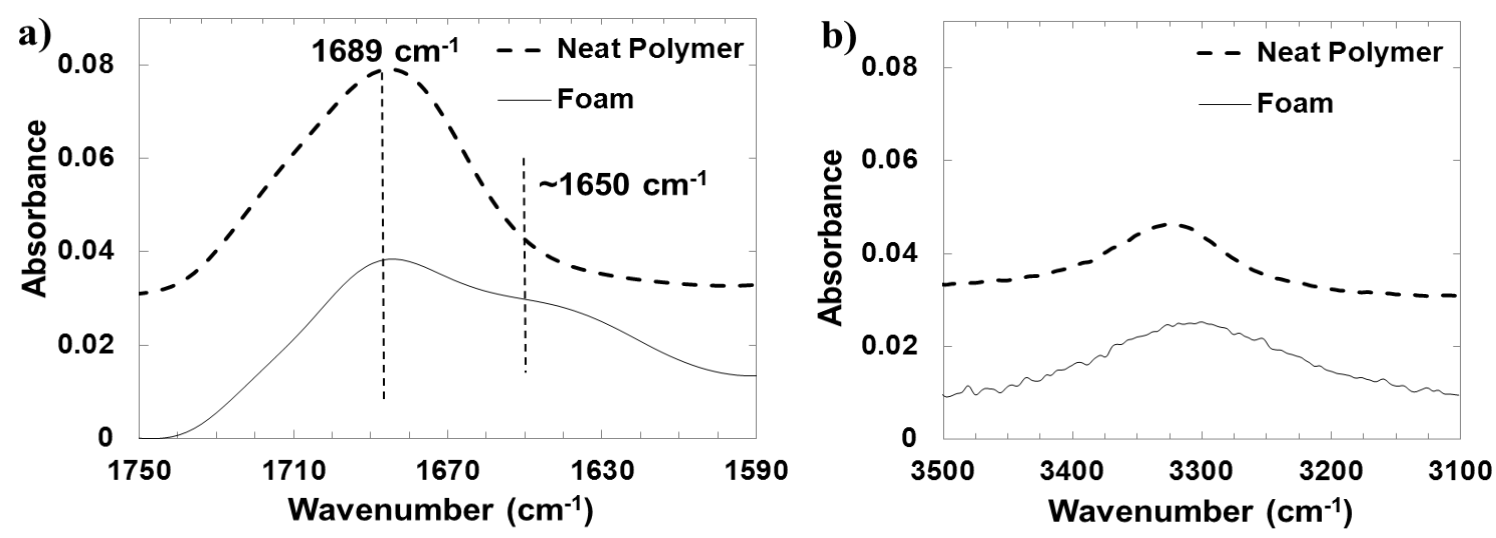

Figure 15: Some important features are observed in the FTIR spectra: 1) presence of a shoulder in the urethane carbonyl bond $\left(1620-1660 \mathrm{~cm}^{-1}\right)$ indicating presence of urea from chemical foaming with water $(b) ; 2)$ presence of hydrogen bonding due to urea groups also shows a broadening of the $\mathrm{N}-\mathrm{H}$ peak $\left(3100-3500 \mathrm{~cm}^{-1}\right)(\mathrm{c})$; and 3$)$ a highly compact and hydrogen bonded structure is estimated as the urethane bond shows a shift to lower wavenumber $\left(1689 \mathrm{~cm}^{-1}\right)$ compared to traditional foams (b). This figure is reproduced with permission from [30]. 
Two important differences are seen between the spectra of the neat polymer and foam: 1) presence of a shoulder in the $1620-1660 \mathrm{~cm}^{-1}$ range indicating presence of urea linkages in the foam from the use of water as a chemical blowing agent (Figure 15a) and 2) a comparatively broader peak from $3100-3500 \mathrm{~cm}^{-1}$ in the foam indicating increased hydrogen bonded N-H vibrations (Figure 15b) [139]. Polyurea segments are reported to show characteristic absorption at $1690-1700 \mathrm{~cm}^{-1}$ in free form, $1660-1670 \mathrm{~cm}^{-1}$ in disordered hydrogen bonded form, and $1630-1645 \mathrm{~cm}^{-1}$ in ordered hydrogen bonded form [136]. The urea shoulder suggests a peak at $\sim 1650 \mathrm{~cm}^{-1}$ (Figure 15a) indicating presence of hydrogen bonding in the urea segments, but not quite as strong as that found in ordered urea segments. This may be due to the high crosslink density that can interfere with the alignment of urea bonds, and prevent formation of as strong a hydrogen bonding as is typically found in the hard segments of polyurethanes.

\subsection{DSC}

DSC curves of these foams are shown in Figure 16. Results show that glass transition/actuation temperature $\left(T_{g}\right)$ could be precisely controlled in the functional range of $44-69^{\circ} \mathrm{C}$ by varying the ratio of HPED to TEA in the foam formulation (Figure 16, Table 4). A higher content of HPED gave a higher $T_{g}$. This agrees well with the results of Wilson et al.,[110] and can be explained from the secondary hydroxyl group of HPED, which positions a methyl group adjacent to the urethane bond. This methyl group can present steric hindrance to rotational motion around the urethane linkage and increase the material $T_{g}$. Also HPED has a functionality of four which gives a higher 
crosslink density to the polymer structure, and can increase its $T_{g}$ in comparison to the trifunctional TEA. However, the transition values don't exactly match the respective compositions in Wilson et al. [110]. This may be due to the use of water as a chemical blowing agent, which introduces urea groups in the network and may increase the $T_{g}$. Also, the as synthesized foam samples can have some residual foaming additives, such as surfactants and catalysts, that can possibly lead to a decrease in $T_{g}$ by plasticization.

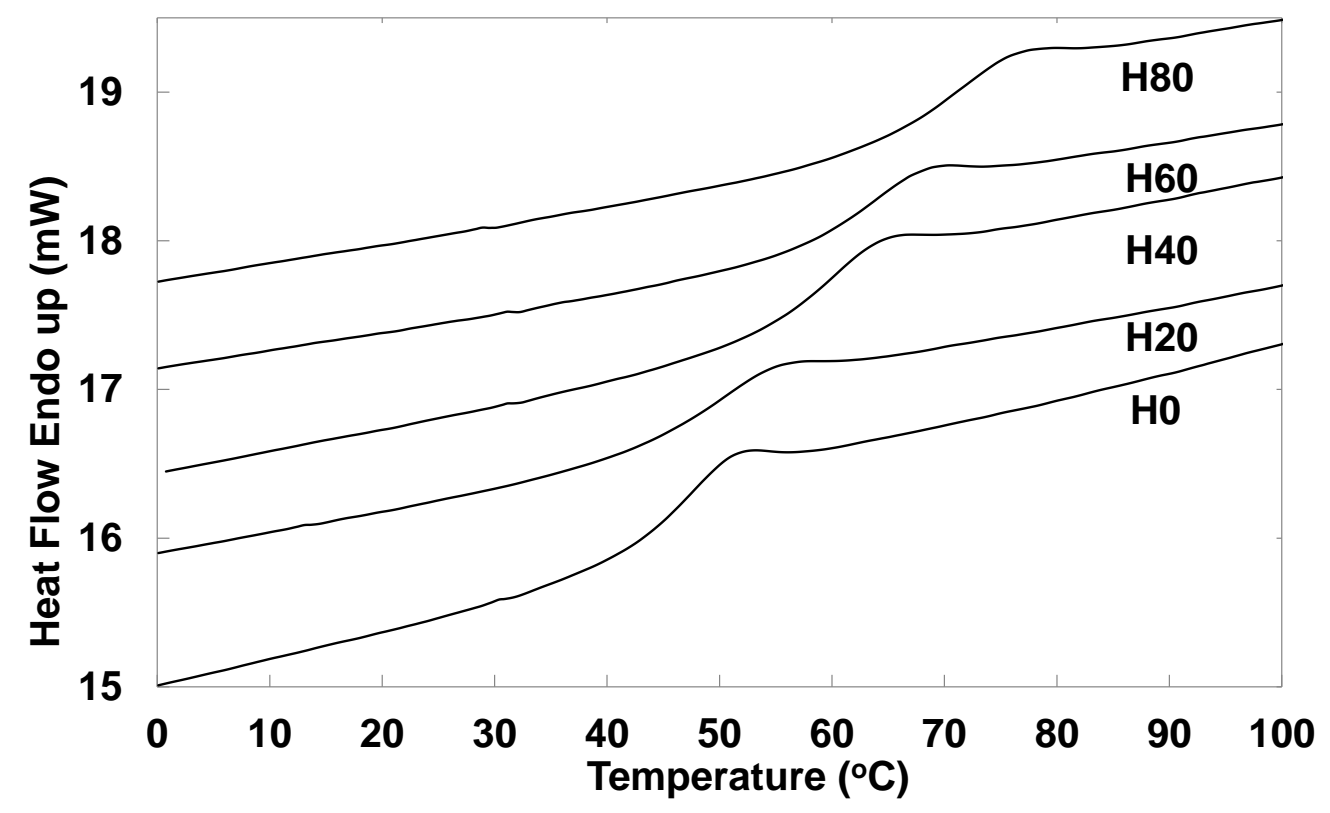

Figure 16: DSC curves for each foam formulation. A variation in $T_{g}$ of approximately $45-70{ }^{\circ} \mathrm{C}$ was achieved by varying the ratio of HPED to TEA in the foam formulation. A higher HPED content gave a higher glass transition value. This is likely due to the increase in rotational steric hindrance due to secondary hydroxyl, and increase in the crosslink density due to the use of tetrafunctional HPED. This figure is reproduced with permission from [30]. 


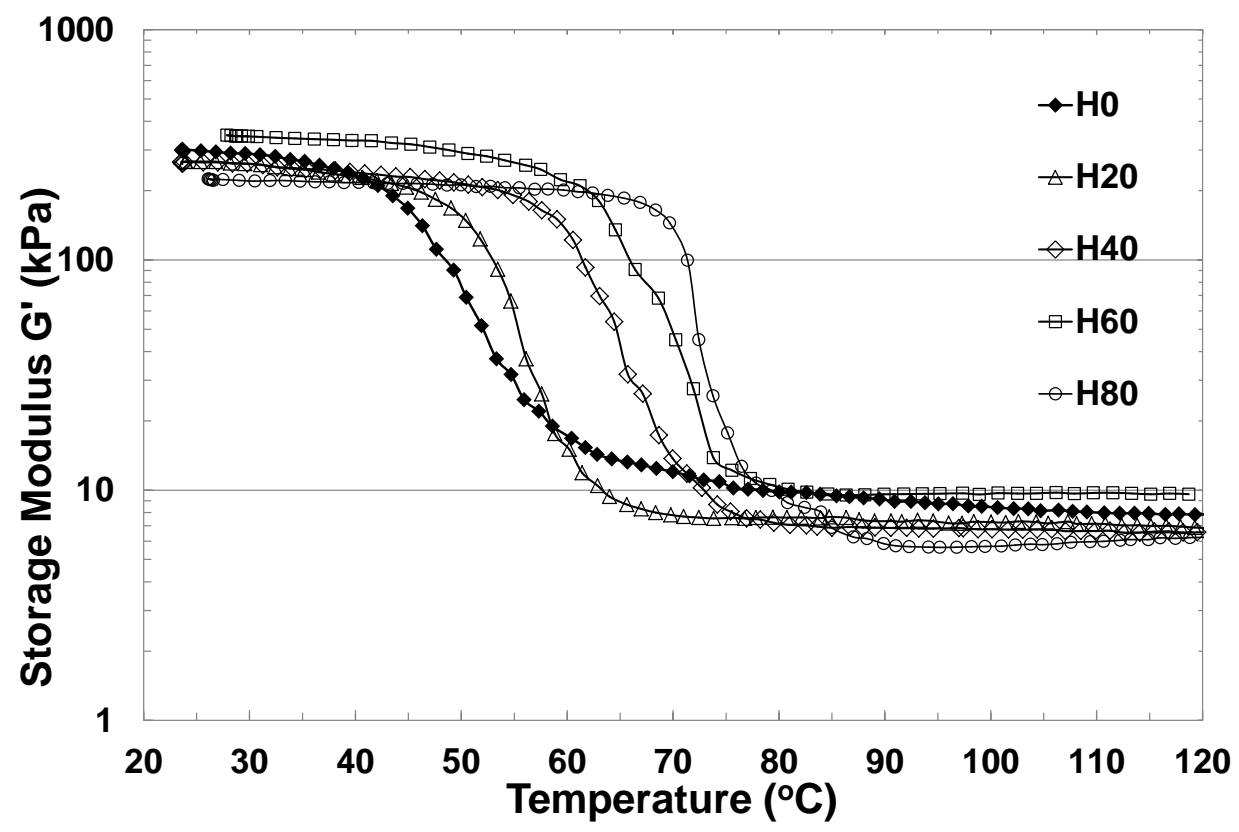

Figure 17: DMTA curves of H0-H80 samples. Variation in transition temperatures is in agreement with the DSC results. This figure is reproduced with permission from [30].

\subsection{DMTA}

The dynamic temperature ramp test results are shown in Figure 17. A drop in modulus is observed across the $T_{g}$ of the foam, going from $200-300 \mathrm{kPa}$ in the glassy state to $5-15 \mathrm{kPa}$ in the rubbery state. Values of $G^{\prime}$ in the rubbery and glassy states, $T_{\text {onset }}$ (temperature at the intersection of the baseline and leading edge of the peak in $\tan \delta$ ), $T_{\delta s}$ (temperature at the peak in $\tan \delta), \Delta T$ (breadth of transition $=2\left(T_{\delta s}-T_{\text {onset }}\right.$ ) according to Yakacki et al.[140]) and $\tan \delta$ values at $T_{\delta s}$ for all formulations are given in Table 4. The rubbery state modulus of shape memory polymers is a measure of force of recovery on actuation, and the ratio of the glassy state to the rubbery state modulus, defined as $G^{\prime}\left(T_{g}-20^{\circ} C\right) / G^{\prime}\left(T_{g}+20{ }^{\circ} C\right)$, is an indicator of the shape fixity of an SMP [110]. The 
modulus of these foams drops by $\sim 30$ times across the transition. While this is not as high as reported for other SMPs in general, shape memory behavior is still quite strong for these foams (Figure 18 and Figure 19), particularly for such low densities. A tradeoff that comes with the high modulus values and highly crosslinked network structure of these foams is the limited elongation at failure ( $35 \%$ for neat unfoamed films) [110]. But with the typically polyhedral shape of the foam cells, individual cell struts undergo only small tensile strains during compression, as they are made to bend and align together. Hence the low net elongation to failure is acceptable for the proposed applications of these foams.

It is noteworthy that the shear storage modulus is found to approximately scale with the density in accordance with $G_{\text {porous }}^{\prime}=G_{\text {neat }}^{\prime}\left(\rho_{\text {porous }} / \rho_{\text {neat }}\right)^{2}$ in the glassy regime, which has been shown to be true for ideal open cell foams $[111,141]$. Here $\rho_{\text {neat }} \sim 1.174 \mathrm{~g} \cdot \mathrm{cm}^{-3}, G_{\text {neat }}^{\prime}=700-905 \mathrm{MPa}$, and average $\rho_{\text {porous }} \sim 0.019 \mathrm{~g} \cdot \mathrm{cm}^{-3}$, yielding calculated $G_{\text {porous }}^{\prime}=183-237 \mathrm{kPa}$. This result is in good agreement with the measured average $G_{\text {porous }}^{\prime} \sim 212-260 \mathrm{kPa}$, (Here the neat polymer density and modulus are taken from the corresponding formulations in Wilson et al. [110]. It is to be noted that the neat polymer density and modulus do not account for the effect of water in the foam composition which affects the chemical crosslink density of the material. However, the actual neat density and modulus may not be very different for the foam material due to strong hydrogen bonding of urea groups which compensates for the loss of chemical crosslinks indicating that these foams have primarily an open cell behavior from a mechanical standpoint. This is consistent with the cell structure images, and confirms 
that although cell membranes are present they are sufficiently thin and do not affect the mechanical behavior of the foam significantly.

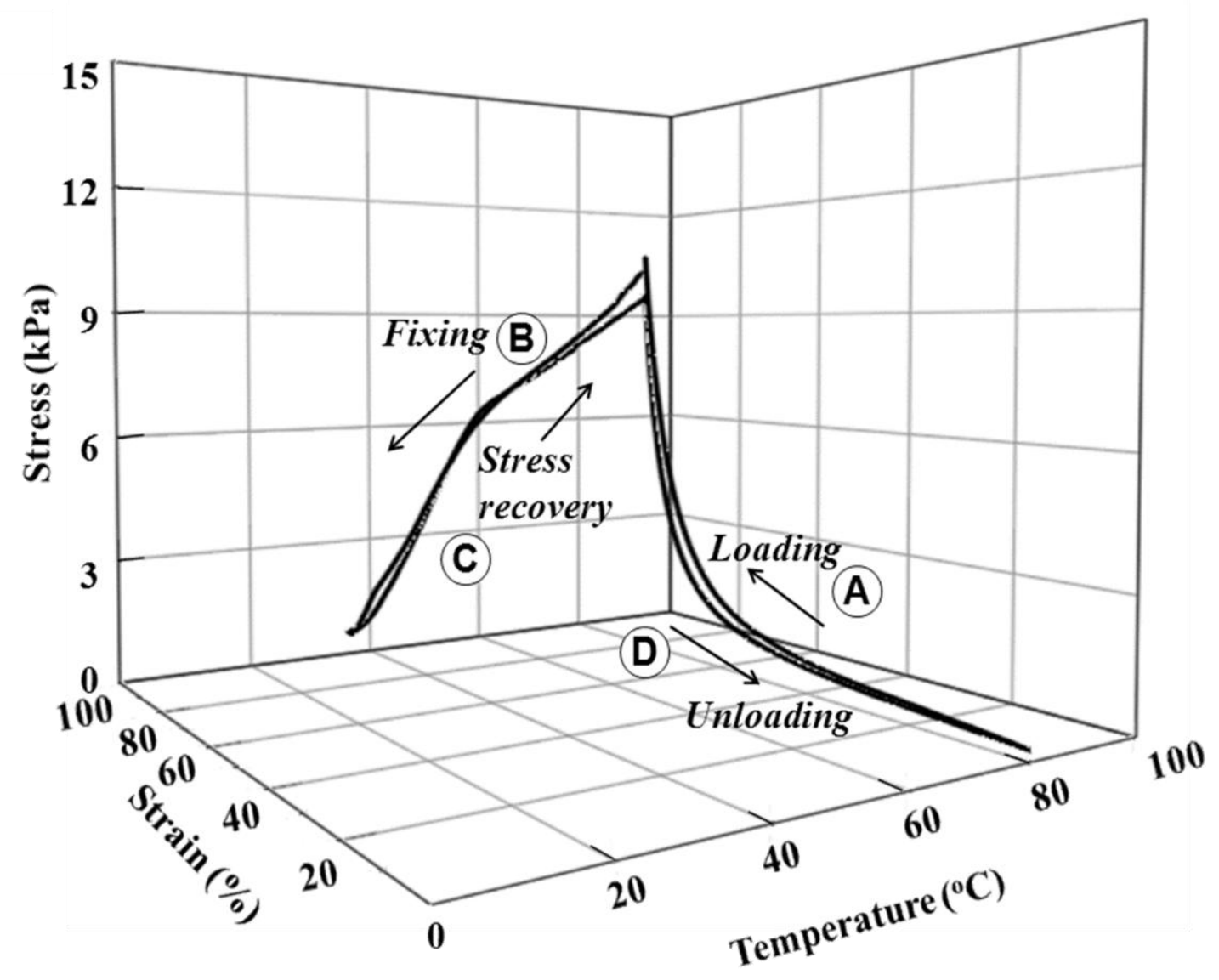

Figure 18: Shape memory cycles performed on a $\mathrm{H} 20$ foam sample $\left(T_{g} \sim 50{ }^{\circ} \mathrm{C}\right)$ in a constrained stress recovery mode. (a) Four steps of shape memory cycle are shown: (A) Loading: The sample heated up to $T_{g}+30{ }^{\circ} \mathrm{C}$ and an $80 \%$ compressive strain applied. (B) Fixing: The compressed sample cooled down to $T_{g}-20{ }^{\circ} \mathrm{C}$. (C) Stress Recovery: Sample heated to $T_{g}+30{ }^{\circ} \mathrm{C}$. (D) Unloading: Strain released at $T_{g}+30{ }^{\circ} \mathrm{C}$. This figure is reproduced with permission from [30]. 

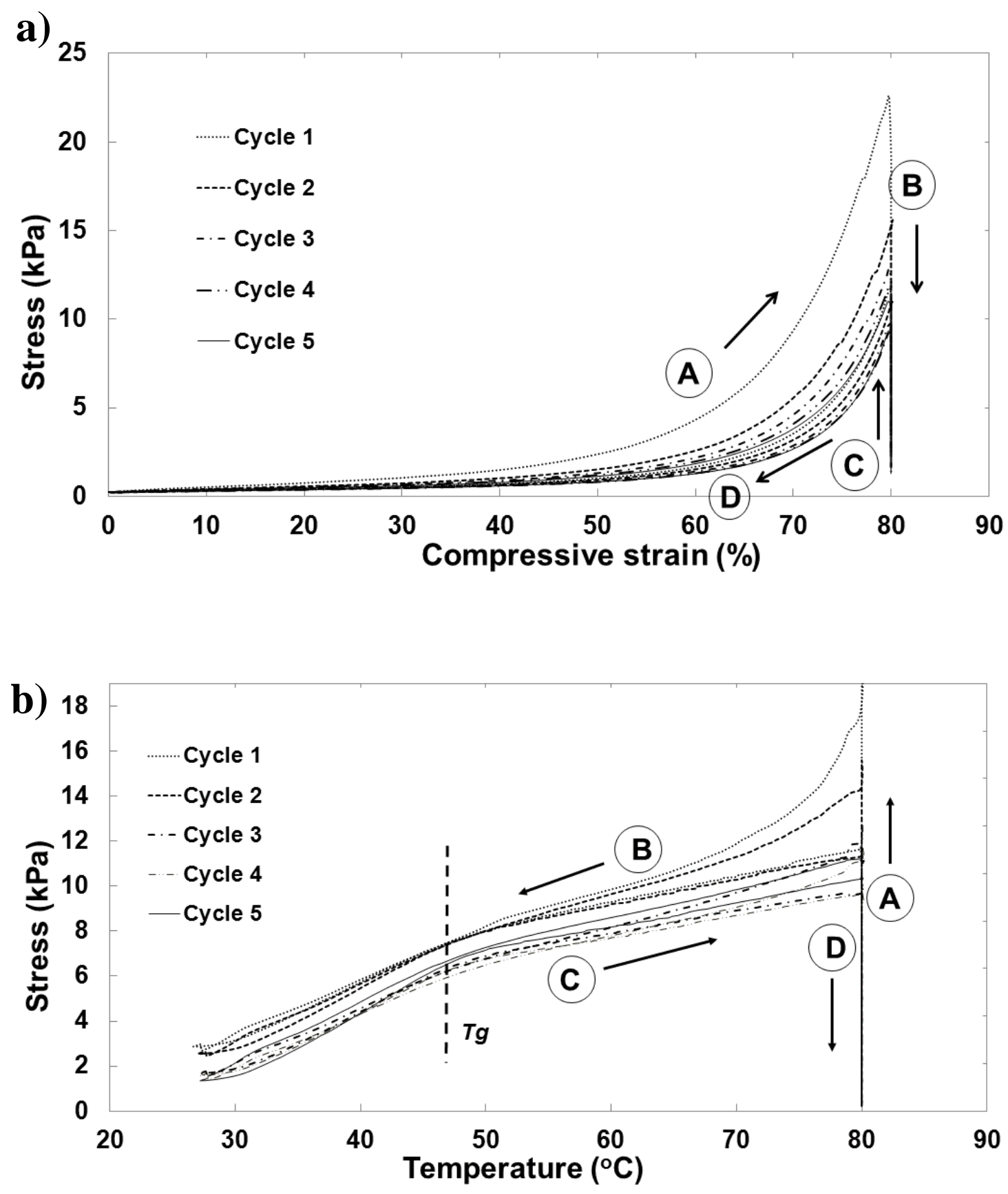

Figure 19: (a) The stress vs. strain behavior of the foams for the 5 cycles. (b) The stress vs. temperature behavior of the foams for 5 cycles. Stages (A), (B), (C) and (D) on plots represent the steps of loading, fixing, stress recovery and unloading respectively. This figure is reproduced with permission from [30]. 


\section{Shape Memory Behavior}

\subsection{Thermomechanical Cycle}

Five shape memory cycles on a H20 foam sample are shown in Figure 18 and Figure 19. A typical shape memory response is seen in a cycle of four key steps, as depicted in Figure 18. The first step of Loading shows an increase in stress as the sample is deformed to $80 \%$ compressive strain at $T_{g}+30{ }^{\circ} \mathrm{C}$. The second step of Fixing reduces the stress rapidly across the $T_{g}$ as the sample is cooled from $T_{g}+30{ }^{\circ} \mathrm{C}$ to $T_{g}-20{ }^{\circ} \mathrm{C}$. The third step of constrained Stress recovery increases the stress back up to $\sim 10 \mathrm{kPa}$ via heating to $T_{g}+30{ }^{\circ} \mathrm{C}$. Finally the fourth step of unloading reduces the stress to zero as the strain is removed, and the foam recovers its primary shape.

Figure 19a and Figure 19b show the behavior of stress vs. strain and stress vs. temperature for 5 shape memory cycles on these foams, respectively. The first cycle shows the highest stress values, followed by the second cycle, and thereafter all the cycles show significant overlap. Hysteresis in the initial cycles is presumed to be from the changes in the macrostructure of foams, such as breaking of some cell membranes or damage to cell struts. Also changes at the microstructural level due to residual stresses in the material and re-arrangement of side groups and dangling ends in the polymer can contribute to hysteresis [110]. An average recovery of $92 \pm 2 \%, 95 \pm 3 \%, 96 \pm 1 \%, 97 \pm 1 \%$ and $97 \pm 1 \%$ was measured in cycles 1 through 5 respectively, over 3 samples for H20 samples. For H60 samples these values were $93 \pm 2 \%, 95 \pm 1 \%, 97 \pm 1 \%, 97 \pm 1 \%, 97 \pm 1 \%$ respectively. High strain recovery in later cycles demonstrates close to an ideal spring 
behavior, consistent with the low values of $\tan \delta$ of these foams Table 4 .

The stress values of $\sim 10-22 \mathrm{kPa}$ as seen in Figure 19 may seem low compared to the typical values of polymeric foams. However, their low density of $0.018 \mathrm{~g} \cdot \mathrm{cm}^{-3}$ as well as rubbery state should be considered. By calculating the corresponding stresses for these foams at higher densities using known scaling relationships, we can compare them to other published SMP foams under similar test conditions. Ashby and Gibson [111, 141] demonstrated that the compressive stresses of a wide range of flexible and rigid polymer foams scale approximately with the material density as follows (valid for open cell foams with $\left.\frac{\rho_{\text {porous }}}{\rho_{\text {nent }}}<0.3\right)[111,141]$ :

$$
\sigma_{a l} \propto\left(\frac{\rho_{\text {yorous }}}{\rho_{\text {ment }}}\right)^{2} ; \sigma_{\mathrm{pl}} \propto\left(\frac{\rho_{\text {porous }}}{\rho_{\text {ment }}}\right)^{\frac{\pi}{2}} \text {, where } \sigma_{e l} \text { and } \sigma_{\mathrm{pl}} \text { are the stresses in elastic }
$$

and plastic deformation regimes, and $\rho_{\text {porows }}$ and $\rho_{\text {neat }}$ are densities of porous and neat forms of polymer, respectively. As the strain of shape memory materials is largely recoverable, the compressive stresses should be valid in the non-linear elastic deformation regime (i.e. $\sigma_{a l}$ ), but both $\sigma_{a l}$ and $\sigma_{p l}$ are considered here for a more complete comparison.

A commercial thermoset epoxy foam (density $0.2 \mathrm{~g} \cdot \mathrm{cm}^{-3}$ ), for instance, was reported to have stress of $\sim 38 \mathrm{kPa}$ at $60 \%$ compressive strain at $30{ }^{\circ} \mathrm{C}$ above $\mathrm{Tg}$ [142]. Compressive stress of the foam reported here is $\sim 4.5 \mathrm{kPa}$ under similar conditions (Figure 19a). As a comparison, values of $\sigma_{e l}$ and $\sigma_{p l}$ for these foams at $0.2 \mathrm{~g} \cdot \mathrm{cm}^{-3}$ density would scale to $\sim 556 \mathrm{kPa}$ and $167 \mathrm{kPa}$ based on the above relations. Polyether polyol based polyurethanes reported by Domeier et al. (density $0.21 \mathrm{~g} \cdot \mathrm{cm}^{-3}$ ) showed 
peak recovery stresses in the range of $1000 \mathrm{kPa}$ on $80 \%$ compressive strain at $T_{g}$ [24]. Stresses for the foams reported here are $\sim 15 \mathrm{kPa}$ under similar conditions except that compression for these was done at $T_{g}+30{ }^{\circ} \mathrm{C}$. This would scale to $\sigma_{e l} \sim 2000 \mathrm{kPa}$ and $\sigma_{p l} \sim 598 \mathrm{kPa}$ for a density of $0.21 \mathrm{~g} \cdot \mathrm{cm}^{-3}$. A polyether polyol series polyurethane foam from Mitsubishi Industries, MF6020, (density of approximately $0.032 \mathrm{~g} \cdot \mathrm{cm}^{-3}[6]$ ) reported about $48 \mathrm{kPa}$ compressive stress at $80 \%$ strain at $30{ }^{\circ} \mathrm{C}$ above $T_{g}$ [21]. The foams reported here have stresses of $\sim 15 \mathrm{kPa}$ under similar conditions, and would scale to $\sigma_{e l} \sim 47 \mathrm{kPa}$ and $\sigma_{p l} \sim 36 \mathrm{kPa}$, respectively. Hence after accounting for the low densities, recovery stresses of these materials are seen to be significantly higher or comparable to other reported SMP foams. It is noteworthy that the number of cycles, strain rate, compressive strain and temperature can all have an effect on the compressive stresses measured for a foam sample. Hence while these results give an estimate of the expected stress range, their exact values could change with changes in the processing conditions.

\subsection{Effect of Storage Temperature on Shape Recovery}

The stress response of $\mathrm{H} 20$ foam samples, as they were held under compression over a 2 hour period, at different temperatures is shown in Figure 20. In the first step of loading to $80 \%$ compressive strain at $T_{g}+30{ }^{\circ} \mathrm{C}$, all four samples showed a similar increase in stress, as expected (Figure 20a). In the second step of hold, the samples were taken to their respective test temperatures of $T_{g}-30{ }^{\circ} \mathrm{C}, T_{g}, T_{g}+30{ }^{\circ} \mathrm{C}$ and $T_{g}+60{ }^{\circ} \mathrm{C}$, and an initial stress relaxation was seen (Figure 20b). The extent of this relaxation was 
higher for samples held above $T_{g}$ compared to the sample held at $T_{g}$. This is expected from the higher mobility of polymer chains at higher temperatures. The sample held at $T_{g}-30{ }^{\circ} \mathrm{C}$ also showed a large drop in stress, which can be explained with the thermal contraction and storage of internal energy and entropy [107] in the glassy state of the polymer, akin to the fixing step of a shape memory cycle. Beyond the initial relaxation, the stresses were held largely constant throughout the hold of 2 hours at the test temperature (Figure 20b). Finally, in the third step of unloading, a decrease in stress was recorded as the strain was released at $T_{g}+30{ }^{\circ} \mathrm{C}$ (Figure 20c). An initial increase in stress was noticed for the sample stored at $T_{g}-30{ }^{\circ} \mathrm{C}$ during strain recovery, possibly due to a lag in the heating of the sample even as the crosshead moved to release the strain. A net shape recovery of $94,93,96$, and $98 \%$ was measured for $\mathrm{H} 20\left(T_{g} \sim 50{ }^{\circ} \mathrm{C}\right)$ samples held at temperatures of $T_{g}+60{ }^{\circ} \mathrm{C}, T_{g}+30{ }^{\circ} \mathrm{C}, T_{g}$, and $T_{g}-30{ }^{\circ} \mathrm{C}$, respectively. For $\mathrm{H} 60$ $\left(T_{g} \sim 60{ }^{\circ} \mathrm{C}\right)$ samples these values were $95,91,98$ and $96 \%$ respectively. $\%$ shape recovery after holding under $80 \%$ compressive strain for 2 hours at $T_{g}+60{ }^{\circ} \mathrm{C}$, for all the compositions, is reported in Table 4. 
a) Compression $\left(\mathrm{Tg}+30^{\circ} \mathrm{C}\right)$

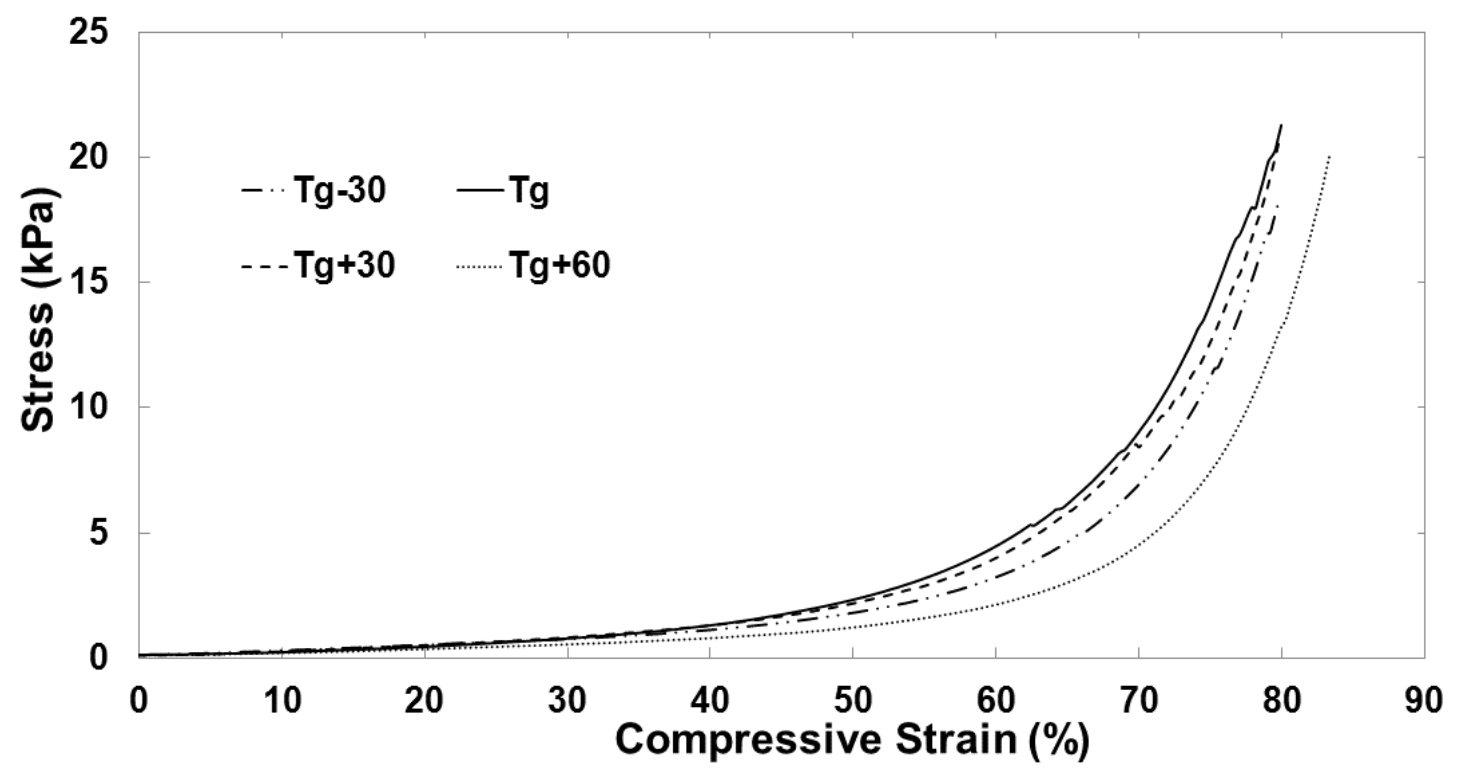

b) $\mathbf{2}$ hour hold

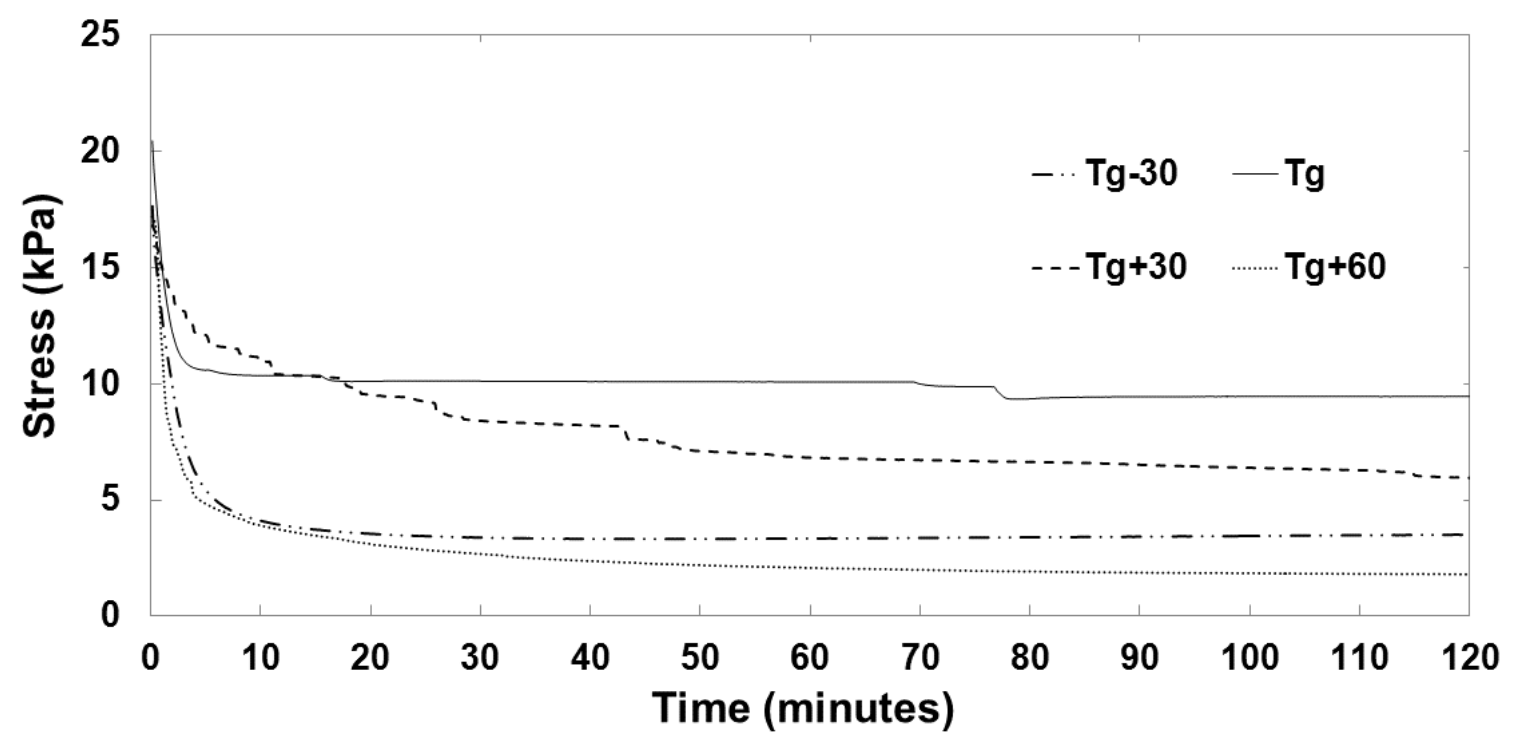




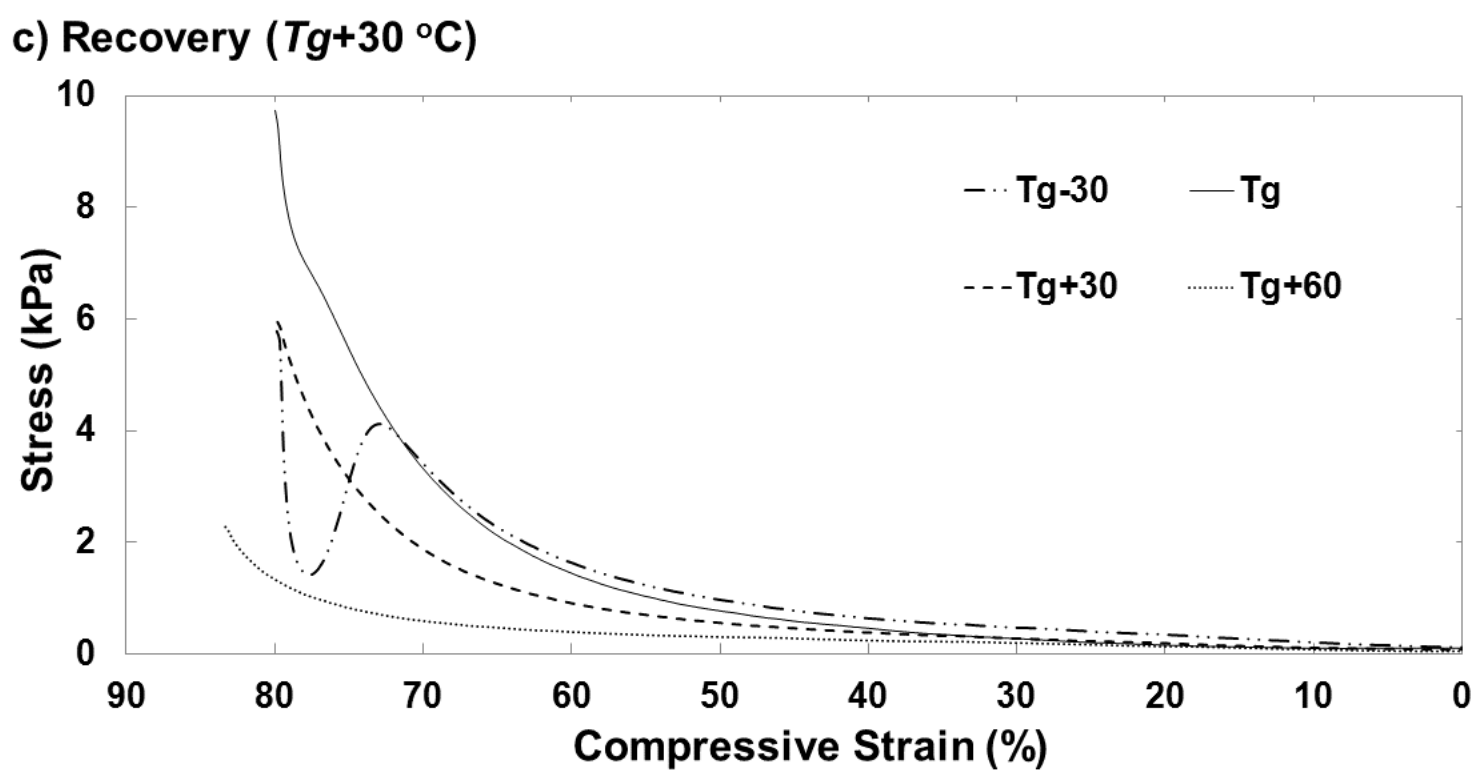

Figure 20: Shape holding behavior of four $\mathrm{H20}$ foam samples. Four samples named $T_{g}-30, T_{g}, T_{g}+30$ and $T_{g}+60$ are (a) deformed to a $80 \%$ compressive strain at $T_{g}+30^{\circ} \mathrm{C}$ (b) held at specific temperatures of $T_{g}-30{ }^{\circ} \mathrm{C}, T_{g}, T_{g}+30{ }^{\circ} \mathrm{C}$ and $T_{g}+60{ }^{\circ} \mathrm{C}$ respectively for a period of two hours and (c) unloaded at $T_{g}+30^{\circ} \mathrm{C}$. This figure is reproduced with permission from [30].

In contrast to the results above, physically crosslinked materials, such as polyether polyol based MF6020 $\left(T_{g} \sim 60{ }^{\circ} \mathrm{C}\right)$ foams [21], have been reported to show $\sim 8-10 \%$ residual strain for hold at $T_{g}+30{ }^{\circ} \mathrm{C}$, and $\sim 100 \%$ residual strain $(0 \%$ shape recovery) for hold at $T_{g}+60{ }^{\circ} \mathrm{C}$ for 2 hours at $80 \%$ compressive strain [21]. Generally, as the applied strain and storage temperature decreased, the extent of secondary-shape forming was seen to decrease for these physically crosslinked foams. Also, Di Prima et al. reported $\sim 40 \%$ strain recovery after compression to $80 \%$ strain at $125{ }^{\circ} \mathrm{C}$ $\left(\sim T_{g}+33^{\circ} \mathrm{C}\right)$ in proprietary epoxy based foams [142]. The foams based on a covalently 
crosslinked network reported here show significantly higher shape recovery in comparison.

Assuming that the above materials are thermally stable at the tested temperatures, this can be explained from the effect of covalent crosslinks on polymer chain relaxation $[107,109]$. The covalent crosslinks in this system can be considered permanent over the conditions and time scales investigated. While network chain segments can conformationally rearrange above $T_{g}$, their ends remain attached to the same network junction point. This constrains the equilibrium arrangement of crosslink points and thus the equilibrium shape of the material. In contrast, the physical crosslinks, such as in segmented polyurethanes, are labile. Here, entropically driven polymer chains can move in and out of the physical crosslinks to achieve a more favorable, lower energy state $[108,143]$. As the rate of this conformational rearrangement of chains is dependent on their mobility, it would occur over increasingly long time scales as temperature is decreased below $T_{g}$, and at shorter time scales at temperatures above $T_{g}$ where the mobility of the chains is high. As the polymer chains relax/re-arrange to an equilibrium conformation while the material is held in the secondary shape, the memory of the primary shape is erased, and a residual strain or phenomenon of secondary-shape forming is observed. Hence, the high shape recovery of the reported materials, after storage at $T_{g}+60{ }^{\circ} \mathrm{C}$ for 2 hours, is consistent with their network structure comprising high density of covalent crosslinks.

However, if a chemical reaction occurs in the material at high temperatures, new bonds can form resulting in loss of shape memory of the polymer for both physically and 
covalently crosslinked materials. Based on the visible discoloration and increase in urealike linkages observed via FTIR (data not shown), such thermally driven chemical changes are thought to cause the relatively low shape recovery of some samples held at high temperatures. While SMPs are not meant to be stored at temperatures above their $T_{g}$, the improved shape holding of these materials at temperatures above $T_{g}$, compared to the other physically crosslinked foams, suggests an advantage of improved shelf life during storage in their secondary shape below $T_{g}$.

\subsection{Maximum Volume Expansion}

Volume expansion measured for the foam samples are reported in Table 4. Cylindrical foam samples $6 \mathrm{~mm}$ in diameter were radially compressed (2-D deformation) to an average minimum diameter of $0.68 \mathrm{~mm}$. On recovery, close to the theoretical volume expansion of up to an average of 68 times was seen. This is an improvement of more than a factor of two compared to other reported shape memory polymer foams [20, 21]. Figure 21 demonstrates the change in the physical form of a foam sample as it expands in a fluid media from a fully compressed shape.

\section{Biocompatibility Characterization*}

Results of the in vitro biocompatibility study of these foams are reported in Table 5. Biocompatibility was analyzed based on the level of cytokine production from the cells cultured on the foam and control substrates. The positive controls (PHA- and LPS-stimulated cells) demonstrated significantly higher cell activation compared to the

*The biocompatibility study was performed by Dr. Judy Van de Water and group at the campus of University of California, Davis, and their support for this work is gratefully acknowledged. 
media alone $(\mathrm{p}<0.05)$. Activation of IL-1 $\beta$, IL-6, IL-8, and TNF- $\alpha$ for the foam was not significantly higher than that for the media alone ( $p>0.05$ in all cases), though average values appear somewhat elevated for IL-1 $\beta$, IL-6, and IL-8. Activation of IL-12, although significantly different compared to the media alone $(\mathrm{p}<0.05)$, was only marginally higher. Overall, the relatively low cell activation induced by the foam suggests promising biocompatibility. These in vitro results are supported by a recent publication that includes pilot 90-day implant results of a related foam formulation, in a vein pouch carotid aneurysm model (porcine) [54]. The in vivo study showed low inflammation and good healing in two foam-treated aneurysms in a single animal.

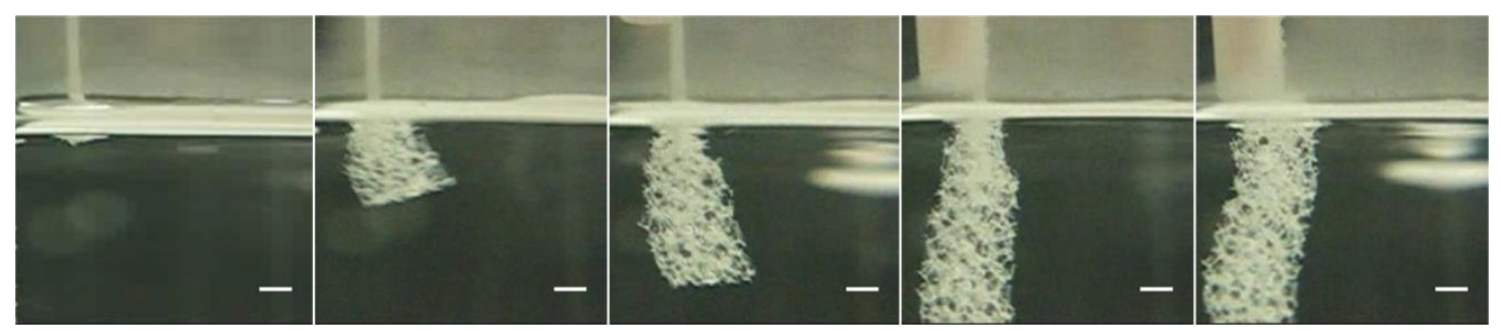

Figure 21: Demonstration of shape memory behavior of a foam. A $6 \mathrm{~mm}$ cylindrical sample of $\mathbf{H 6 0}$ foam was compressed in a mechanical crimper above its $T_{g}$ and then allowed to cool down to fix the compressed shape. The compressed foam was taped to a rod (seen in the last two panes) and then plunged in a water bath heated to $80^{\circ} \mathrm{C}$. Actuation is seen immediately on contact with water and up to 70 times expansion in volume is observed. Approximate time between panes is 1 second. Scale bar is $\mathbf{2 . 5} \mathbf{~ m m}$. This figure is reproduced with permission from [30]. 
Table 5: Results of the biocompatibility study of the foam. This table is reproduced with permission from [30].

\begin{tabular}{|c|c|c|c|c|c|}
\hline \multirow{2}{*}{ Material } & \multicolumn{5}{|c|}{ Cytokine Concentration $\left(\mathrm{pg} \cdot \mathrm{mL}^{-1}\right)^{\mathrm{a}}$} \\
\hline & IL-1及 & IL-6 & IL-8 & IL-12 & TNF- $\alpha$ \\
\hline media only & $2 \pm 1$ & $9 \pm 7$ & $846 \pm 896$ & $29 \pm 21$ & $97 \pm 224$ \\
\hline PHA & $\begin{array}{c}106 \pm 72 \\
(p=0.00075)\end{array}$ & $\begin{array}{l}414 \pm 125 \\
(p=1.6 e-6)\end{array}$ & $\begin{array}{c}17556 \pm 15030 \\
(p=0.0028)\end{array}$ & $\begin{array}{c}83 \pm 69 \\
(p=0.015)\end{array}$ & $\begin{array}{l}310 \pm 295 \\
(p=0.012)\end{array}$ \\
\hline LPS & $\begin{array}{c}254 \pm 48 \\
(p=2.2 e-8)\end{array}$ & $\begin{array}{c}529 \pm 21 \\
(p=5.6 e-15)\end{array}$ & $\begin{array}{c}57159 \pm 33715 \\
(p=0.00025)\end{array}$ & $\begin{array}{c}511 \pm 326 \\
(\mathrm{p}=0.00062)\end{array}$ & $\begin{array}{l}939 \pm 330 \\
(p=4.4 e-5)\end{array}$ \\
\hline Foam & $\begin{array}{c}17 \pm 45 \\
(p=0.16)\end{array}$ & $\begin{array}{l}64 \pm 173 \\
(p=0.17)\end{array}$ & $\begin{array}{c}3689 \pm 8839 \\
(p=0.15)\end{array}$ & $\begin{array}{c}30 \pm 22 \\
(p=0.014)\end{array}$ & $\begin{array}{l}100 \pm 213 \\
(p=0.24)\end{array}$ \\
\hline
\end{tabular}

${ }^{a}$ mean \pm standard deviation $(n=10)$

\section{Conclusion}

Biostable foams with sharp single glass transitions $\left(T_{g}\right)$ customizable in the functional range of $45-70{ }^{\circ} \mathrm{C}$ were achieved, with about 30 times reduction in storage modulus across the transition. Thermomechanical testing confirmed strong shape memory behavior with $97-98 \%$ shape recovery over repeated cycles, a glassy storage modulus of 200-300 kPa and recovery stresses of 5-15 kPa. Shape holding tests under constrained storage above the $T_{g}$ suggested improved shelf-life, and a high volume expansion of up to 70 times was seen on actuation of these foams from a fully compressed state. Further, low in vitro cell activation induced by the foam, relative to controls, suggested promising biocompatibility. These porous polymeric scaffolds constitute an important class of novel smart biomaterials with multiple potential applications. 


\title{
CHAPTER III
}

\section{CONTROLLING THE ACTUATION RATE OF SHAPE MEMORY POLYMER}

\author{
FOAMS IN WATER*
}

Chapters I and II established the significant potential utility of SMP foams in embolic biomedical application. Despite their unique properties, the use of SMP foams in practical applications is limited by a key issue: these materials have been observed to undergo a loss of their ability to "actuate on demand" in certain conditions. This loss of "on demand" actuation may happen by: (a) loss of the material's tendency to actuate following storage in a secondary shape over long periods of time or at relatively high temperatures (referred to as secondary-shape forming [21] and addressed in Chapter I [30]) and/or (b) premature actuation, such as on exposure to moisture, a plasticizing agent, which depresses the actuation temperature of the foams $[55,137,144]$. The phenomenon of actuation of SMP foams in the presence of moisture, and thereby the loss of their "on demand" actuation, are discussed in this chapter.

The effect of moisture on the actuation of SMPs has been reported previously as an alternate actuation mechanism [145-147]. Huang et al. [145, 146] described several "water actuated" neat/nonporous and porous SMP devices based on the MM 3520, MM 3550, and MM 5510 materials from Mitsubishi Heavy Industries. Jung et al. [147] reported polyurethane SMP films based on poly(ethylene glycol) (PEG), polyhedral oligomeric silsesquioxane (POSS), and 1,4- phenyldiisocyanate. These films had a

*Parts of this chapter are reprinted with permission from Singhal, P. et al. Controlling the Actuation Rate of Low-Density Shape-Memory Polymer Foams in Water. Macromol. Chem. Phys. 2012;(in press) DOI: 10.1002/macp.201200342. Copyright 2012 WILEY-VCH Verlag GmbH \& Co. KGaA, Weinheim. 
hydrophilic soft segment comprising PEG and a hydrophobic hard segment containing POSS. They demonstrated water-responsive shape recovery of more than $70 \%$ for all samples at $30{ }^{\circ} \mathrm{C}$, depending on the POSS content of the films [147]. Also, water responsive hybrid shape memory materials were recently reported in literature [148, 149]. In these materials, a second inorganic phase was used to restrain the recovery of the polymer in the dry state, as opposed to using a polymer phase transition. Yang et al. [137] investigated the mechanism of actuation of SMPs with water on MM 3520 films, and found that it is a combined effect of the weakening of the hydrogen bonding between $\mathrm{N}-\mathrm{H}$ and $\mathrm{C}=\mathrm{O}$ groups and plasticization caused by the small water molecules. SMP foams from our group [30] were tested for the effect of moisture by Yu et al. [55] and again the above mechanisms were found to be at work in quickly depressing the $T_{g}$ of the foam and causing its rapid actuation on exposure to water.

The passive self-actuation of a thermally responsive SMP device in water or physiological media at body temperature may be a useful feature for a simpler device deployment mechanism without a heating capability. However, if the passive actuation occurs too quickly or in an uncontrollable manner, it can cause problems. For example, the usage of SMP foams for minimally invasive treatment of aneurysms requires transcatheter delivery of the device in its compressed cylindrical shape. Once it reaches the target site, it would be actuated to its primary spherical shape to fill the aneurysm and eventually to resist any blood flow inside it. As the compressed device may be exposed to moisture/physiological fluid while inside the catheter, premature expansion of a moisture- sensitive device may occur during the delivery itself. This can drastically 
increase the friction within the catheter and potentially inhibit delivery of the device. With low-density foams, this issue becomes even more important due to the increased surface area available for the uptake of water/moisture. Hence, it is desirable to be able to control the rate of actuation of the foam-based SMP devices in the presence of moisture.

One possible solution to this problem would be to vary the hydrophobicity of the SMP, thereby controlling the extent of the depression in actuation temperature due to exposure to water. To test this hypothesis, we synthesized and characterized a novel series of low density SMP foams with increasing diisocyanate monomer hydrophobicity. Such materials should exhibit a controlled reduction in the rate of their self-actuation in water, facilitating transcatheter delivery of the device. Here, we report the process of synthesis of these materials, their key physical properties, and their actuation behavior. Also, an analysis of the actuation mechanism is presented, and various challenges and potential solutions to the transcatheter delivery of SMP foam devices are discussed.

\section{A. Materials and Methods}

\section{Foam Synthesis}

HPED (99\%; Sigma Aldrich Inc.), TEA (98\%; Alfa Aesar Inc.), HDI (TCI America Inc.), 1, 6 diisocyanato trimethylhexane, 2,2,4- and 2,4,4- mixture (TMHDI, TCI America Inc.), and DI water ( > $17 \mathrm{M} \Omega \cdot \mathrm{cm}$ purity; Millipore water purifier system; Millipore Inc.,) were used as received (Figure 22). Foams were synthesized in a three- 
step method reported previously [30], using the amounts indicated in Table 6, and were allowed to cure for at least a week before testing. The notations 100TM, 80TM, 60TM, 40TM, 20TM, and 0TM denote TMHDI constituting 100, 80, 60, 40, 20, and 0 mol\% of isocyanate, respectively, with the remaining mol\% of isocyanate coming from HDI, as indicated in Table 6 .

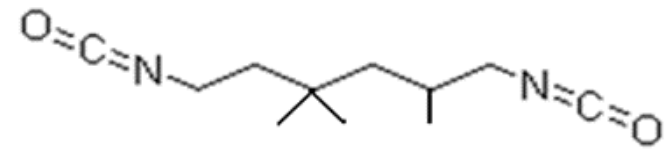

$2,2,4$ (and 2,4,4) Trimethyl 1,6 Diisocyanatohexane (TMHDI)

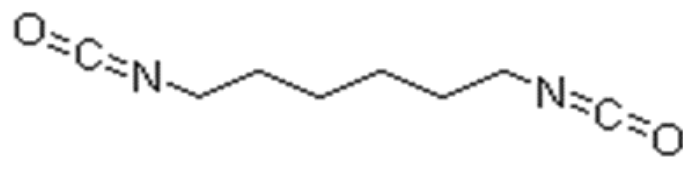

1,6 Diisocyanatohexane(HDI)

Figure 22: Molecular structure of HDI and TMHDI, the two isocyanates used in this study. Methyl groups on the backbone of TMHDI were expected to give it a more hydrophobic character relative to HDI.

Table 6: Composition of foams synthesized using hexamethylene diisocyanate (HDI) and trimethyl hexamethylene diisocyanate (TMHDI) for the isocyanate component of the urethane. This table is reproduced with permission from [29].

\begin{tabular}{|c|c|c|c|c|c|c|c|c|c|c|}
\hline $\begin{array}{c}\text { Sample } \\
\text { ID }\end{array}$ & $\begin{array}{c}\text { HDI } \\
\text { (wt.\%) }\end{array}$ & $\begin{array}{l}\text { TMHDI } \\
\text { (wt.\%) }\end{array}$ & $\begin{array}{l}\text { HPED } \\
\text { (wt.\%) }\end{array}$ & $\begin{array}{c}\text { TEA } \\
\text { (wt.\%) }\end{array}$ & $\begin{array}{l}\text { Water } \\
\text { (wt.\%) }\end{array}$ & $\begin{array}{c}\text { DC5179 } \\
\text { (wt.\%) }\end{array}$ & $\begin{array}{l}\text { DCI990 } \\
\text { (wt.\%) }\end{array}$ & $\begin{array}{l}\text { T-131 } \\
\text { (wt.\%) }\end{array}$ & $\begin{array}{l}\text { BL-22 } \\
\text { (wt.\%) }\end{array}$ & $\begin{array}{c}\text { Enovate } \\
\text { (pph) }\end{array}$ \\
\hline 100TM & 0.00 & 66.79 & 17.11 & 5.73 & 2.35 & 3.81 & 3.30 & 0.26 & 0.64 & 7.62 \\
\hline 80TM & 11.00 & 55 & 17.63 & 5.92 & 2.42 & 3.8 & 3.29 & 0.25 & 0.64 & 7.6 \\
\hline 60TM & 22.70 & 42.58 & 18.18 & 6.09 & 2.49 & 3.78 & 3.28 & 0.25 & 0.64 & 7.57 \\
\hline 40TM & 35.15 & 29.30 & 18.77 & 6.29 & 2.57 & 3.77 & 3.27 & 0.25 & 0.64 & 7.54 \\
\hline 20TM & 48.43 & 15.14 & 19.39 & 6.50 & 2.66 & 3.75 & 3.25 & 0.25 & 0.63 & 7.50 \\
\hline OTM & 62.62 & 0.00 & 20.06 & 6.72 & 2.75 & 3.73 & 3.24 & 0.25 & 0.63 & 7.47 \\
\hline
\end{tabular}

The wt.\% value represents the net amount of the component in the final foamed polymer. 
Neat polymers of corresponding compositions, excluding the physical and chemical blowing agents and other foaming additives (surfactants and catalysts), were synthesized according to Table 7 for contact angle measurements. Calculated amounts of monomers were mixed together and cast in polystyrene dishes. The curing profile involved an initial cure at room temperature for 2 hours followed by ramping up to $120{ }^{\circ} \mathrm{C}$ at $20{ }^{\circ} \mathrm{C} \cdot$ hour $^{-1}$. Thereafter, the temperature was maintained at $120{ }^{\circ} \mathrm{C}$ for 2 hours, and finally the heat was turned off and samples were allowed to cool down to room temperature. An inert nitrogen atmosphere was maintained throughout the curing process.

Table 7: Composition of neat/non-porous polymers synthesized following the same synthesis scheme as that of the foams (Table 1). Amount of TMHDI was gradually increased from 0TM (all HDI) to 100TM (all TMHDI) composition. No foaming additives such as surfactant, catalysts and physical and chemical blowing agents were added. This table is reproduced with permission from [29].

\begin{tabular}{|c|c|c|c|c|}
\hline Sample ID & $\begin{array}{c}\text { HDI } \\
(\mathbf{w t} . \%)\end{array}$ & $\begin{array}{c}\text { TMHDI } \\
(\mathbf{w t .} \%)\end{array}$ & $\begin{array}{c}\text { HPED } \\
(\mathbf{w t .} \%)\end{array}$ & $\begin{array}{c}\text { TEA } \\
(\mathbf{w t} . \%)\end{array}$ \\
\hline $100 \mathrm{TM}$ & 0.00 & 61.65 & 28.72 & 9.62 \\
\hline $80 \mathrm{TM}$ & 10.11 & 50.57 & 29.45 & 9.87 \\
\hline $60 \mathrm{TM}$ & 20.75 & 38.91 & 30.21 & 10.12 \\
\hline $40 \mathrm{TM}$ & 31.95 & 26.63 & 31.02 & 10.39 \\
\hline $20 \mathrm{TM}$ & 43.77 & 13.68 & 31.87 & 10.68 \\
\hline $0 \mathrm{TM}$ & 56.26 & 0.00 & 32.77 & 10.98 \\
\hline
\end{tabular}

The wt.\% value represents the net amount of the component in the final foamed polymer. 


\section{Post-processing of Foams}

Post-processing of foams is often required because the as-blown foams typically retain residual membranes post-synthesis. In order to make foams completely open cell or reticulated, as desired for the proposed application, secondary physical processes such as hydrolysis, oxidation, heat, or mechanical treatment are normally employed [129]. Caustic hydrolysis of polyurethanes, also referred to as "quenching," is a standard process of reticulation of traditional polyurethane foams in industry [150]. Another well accepted method of reticulating polyurethane foams is "zapping," which involves controlled burning of the membranes via saturation with flammable gases [150]. Several patents have been filed in this area of study [151-156], and are discussed in greater detail in Chapter IV. Here, an acid-based etching was performed on the foams prior to testing their actuation and actuation related properties. Although this etching process was not optimized for complete membrane removal in the foams, it was included to account for the possibility that more hydrophilic surfaces may be created from these post-processing etching methods [157]. As increased surface hydrophilicity may alter the moisture sensitivity of the material relative to un-etched foams, the device actuation results with inclusion of the etching process are potentially more relevant to an actual device. For performing the cleaning and etching processes, blocks of foams were etched in hydrochloric acid (0.1 N; BDH Chemicals) for 2 hours. Thereafter they were cleaned with two 15 minute treatments each of $80-20$ vol.\% DI water/contrad solution and DI water, respectively. All steps were performed under sonication. The foams were finally dried overnight under vacuum at $50{ }^{\circ} \mathrm{C}$ before testing. 


\section{Characterization of Physical Properties}

\subsection{Density and Cell Structure}

Core densities of three foam samples of each composition were measured from the representative top and middle sections as per the ASTM standard D-3574. For cell structure characterization, thin slices of foam were cut from a representative top section of the blown foam, and images were captured in the brightfield mode on a Leica MZ8 microscope (Leica Microsystems Inc.) using RSImage Software (Roper Scientific Inc.).

\subsection{DSC}

Glass transition temperature $\left(T_{g}\right)$ was measured using a Q-200 DSC (TA Instruments Inc.). A 3-5 mg sample was compressed to near bulk density and loaded in a vented aluminum pan at room temperature, cooled to $-40{ }^{\circ} \mathrm{C}$ and then run through a heat/cool/heat cycle from -40 to $120{ }^{\circ} \mathrm{C}$ at $10{ }^{\circ} \mathrm{C} \cdot \mathrm{min}^{-1}$. The half-height of the transition during second heat was taken as an estimate of the $T_{g}$.

\subsection{DMTA}

For characterizing the mechanical properties of the foams as a function of temperature, a dynamic temperature ramp test was performed on an ARES-LS2 rheometer (TA Instruments Inc.) according to a previously described method [30]. A torsion rectangle test fixture was used on samples cut to approximately $45 \mathrm{~mm}$ long, 12 $\mathrm{mm}$ wide, and $6 \mathrm{~mm}$ thick with a gap distance of $25 \mathrm{~mm}$. The samples were prepared by 
embedding both ends in a polyurethane neat polymer to prevent damage and slippage of the foam sample in the metal grips. Dynamic temperature ramp tests were then run for each formulation in triplicate, at a frequency of $1 \mathrm{~Hz}$ and constant heating rate of $1{ }^{\circ} \mathrm{C} \cdot \mathrm{min}^{-1}$ from 25 to $150{ }^{\circ} \mathrm{C}$. An initial shear strain of $0.2 \%$ was used. However, as the temperature increased, the strain was adjusted by the control software to maintain a torque range of 0.5 to $5 \mathrm{~g} \cdot \mathrm{cm}$, allowing a maximum strain of $10 \%$ (still within the linear viscoelastic region) at high temperatures. Data points were collected every 5 seconds. Dynamic shear storage modulus $\left(G^{\prime}\right)$, dynamic shear loss modulus $\left(G^{\prime \prime}\right)$, and their ratio $\tan \delta\left(=G^{\prime \prime} / G^{\prime}\right)$ were recorded using the Orchestrator ${ }^{\mathrm{TM}}$ software (TA Instruments Inc.). $T_{\text {onset }}$ was recorded as the temperature at which the baseline and the leading edge of the peak in $\tan \delta$ intersect, $T_{\delta s}$ (another measure of $T_{g}$ ) was recorded as the temperature at which the $\tan \delta$ curve was at its peak value, and $\Delta T$ (breadth of transition) was recorded as twice the difference between $T_{\delta s}$ and $T_{\text {onset }}$, according to Yakacki et al. [140].

\subsection{Fourier Transform Infrared Spectroscopy (FTIR)}

FTIR spectra of the foam were collected using a Spectrum 2000 FTIR (Perkin Elmer Inc.). The sample chamber was purged with nitrogen gas for 5 minutes and a background spectrum was captured. Thereafter, thin slices cut from foam blocks ( 2-4 $\mathrm{mm}$ in thickness) were placed on a $2 \mathrm{~cm}^{-1}$ JStop holder ( $0.88 \mathrm{~cm}$ aperture). The chamber was again purged for 5 minutes after placing the sample, and an FTIR spectrum was collected in the transmission mode at a resolution of $4 \mathrm{~cm}^{-1}$. The test was performed in duplicate to ascertain reproducibility of the spectra. A total of 50 scans were taken for 
each sample and the background spectrum was subtracted using the Spectrum software (Perkin Elmer Inc.).

\section{Characterization of Foam Actuation and Actuation-related Properties}

\subsection{Contact Angle Measurement}

Contact angle measurements were made on neat/unfoamed polymers corresponding to the foam compositions (Table 7) using a DSA100 (Kruss Inc.). DI water drops of 4-5 $\mathrm{ml}$ volume were placed on the sample surface. The drop was allowed to come to an equilibrium shape for 30 seconds and the resulting contact angle was measured using the drop shape analysis software (Kruss Inc.). An average value over ten measurements was calculated for an estimate of the contact angle of the polymer surface.

\subsection{Equilibrium Moisture Uptake}

Cylindrical foam samples were cut from a $2.5 \mathrm{~cm}$ thick foam block using an $8 \mathrm{~mm}$ diameter biopsy punch and dried under vacuum at $50{ }^{\circ} \mathrm{C}$ overnight. The dry sample weight $\left(W_{d r y}\right)$ was measured on an Ohaus Analytical Plus scale (Central Carolina Scale Inc.). The samples were then soaked in $\sim 1000$ times weight excess of water for 24 hours. To measure the equilibrium water absorption, the samples were first taken out from water media and the excess (adsorbed) water on the samples was removed in two steps: (a) repeated pressing (by hand) between sheets of laboratory grade kimwipes 
(Kimberly-Clark Inc.), until no wet spots could be seen, and then (b) pressing between kimwipes to 1 metric ton pressure for 2 minutes in a \#3925 hydraulic press (Carver Laboratory Equipments). The samples were placed on the weighing balance immediately thereafter and their equilibrium weight $\left(W_{w e t}\right)$ was recorded. The absorbed water $(w t . \%)$ in the foam samples was calculated as:

$$
\text { Absorbed water }(w t \%)=\left(W_{\text {wet }}-W_{\text {dry }} / W_{\text {dry }}\right) \times 100
$$

Five samples were tested for each foam composition.

\subsection{Depression in $T_{g}$ with Exposure to Moisture}

Cylindrical samples of foam were cut from a $2.5 \mathrm{~cm}$ thick block using an $8 \mathrm{~mm}$ diameter biopsy punch and submerged in a water bath at $37{ }^{\circ} \mathrm{C}$ for $5,15,30,60$, or 120 minutes. After the specified time of submersion, the samples were taken out and excess water was removed in two steps using kimwipes and a hydraulic press as described above. A DSC scan was then run from -40 to $80{ }^{\circ} \mathrm{C}$ at $10{ }^{\circ} \mathrm{C} \cdot \mathrm{min}^{-1}$ on a $4-8 \mathrm{mg}$ foam sample in a vented aluminum pan, using a Q-200 DSC (TA Instruments Inc.). The $T_{g}$ of the sample was estimated from the half height of the thermal transition during the first heating scan. Five samples of each composition were tested at each time point.

\subsection{Rate of Expansion of Foam in Fluid Media at $37^{\circ} \mathrm{C}$}

Cylindrical foam samples approximately $1 \mathrm{~cm}$ in length were cut using a $6 \mathrm{~mm}$ diameter biopsy punch and threaded over straight $0.09 \mathrm{~mm}$ diameter nitinol wires 
(Nitinol Devices \& Components, Inc.). An initial measurement of the diameter of the punched samples was taken using a digital micrometer. Each sample was then radially compressed as small as possible, at $97{ }^{\circ} \mathrm{C}$ at a radial compression rate of approximately $6.2 \mathrm{~mm} \cdot \mathrm{s}^{-1}$, using an SC150-42 Stent Crimper (Machine Solutions Inc.). The compressed shape was fixed by allowing the sample to passively cool down to room temperature in the compressed state over a period of 2 hours. The samples were allowed to rest in this secondary compressed shape for 24 hours in a nitrogen purged environment before testing. After the resting period, the compressed diameter was measured using a digital micrometer. The nitinol wires holding the compressed foam samples were then strung across a heavy fixture such that the foam never touched the fixture, and the assembly was submerged in a water bath heated to $37.0 \pm 0.5^{\circ} \mathrm{C}$. A scale was submerged alongside the samples. Images of the foam samples were taken at 1 to 5 minute intervals over a period of 1 hour, as the foams expanded in the water bath. For the measurement of the diameter of the foam at any given time, the maximum diameter along the length of the sample was measured from the captured images using ImageJ software. Five samples of each composition were tested. Results were normalized by the measured initial (uncompressed) diameter.

\subsection{Theoretical Solubility Parameter Analysis}

Hoy's solubility parameters were calculated to estimate the relative swelling of the foams in the water media [158]. The calculations were done on the basis of the four types of network units generated from the reaction of isocyanate with HPED, TEA, 
water, and the excess isocyanate. Although the excess isocyanate groups can undergo several secondary reactions such as allophanate or biuret formation, for simplicity they were assumed to be converted to primary amines from reaction with ambient moisture.

Group contributions of each of these network units, HPED, TEA, water, and excess isocyanate (Figure 23), were calculated as per Van Krevelen et al. [158]. The net solubility parameter components $\delta_{t}, \delta_{p}$, and $\delta_{h}$ representing total, polar, and hydrogen bonding components of the polymer cohesive energy density, were then determined by adding the respective contributions of the network units on a volume fraction $(\vartheta)$ basis (Equation 3.2). The dispersion force component of the total solubility parameter was calculated using Equation 3.3.

$$
\begin{aligned}
& \delta=\delta_{H P E D} * \vartheta_{H P E D}+\delta_{T E A} * \vartheta_{T E A}+\delta_{\text {water }} * \vartheta_{\text {water }}+\delta_{\text {Excess }} * \vartheta_{\text {excess }} \\
& \text { here } \delta=\delta_{t} \text { or } \delta_{p} \text { or } \delta_{k} \\
& \delta_{d d}=\sqrt{\left(\delta_{t}^{2}-\delta_{p}^{2}-\delta_{k}^{2}\right)}
\end{aligned}
$$




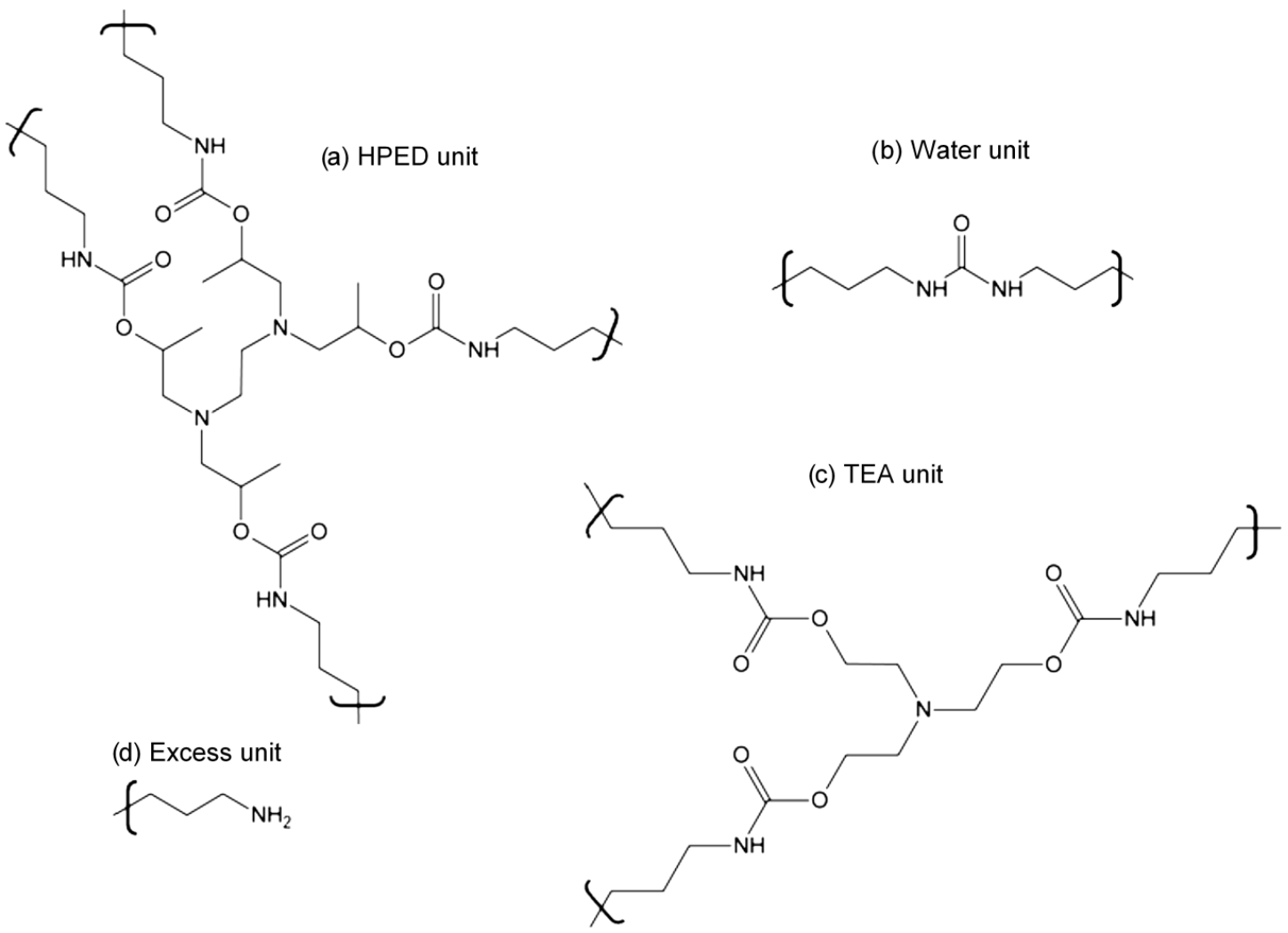

Figure 23: Structures of the (a) HPED, (b) TEA, (c) water, and (d) excess network units used to calculate the contributions of Hoy's solubility parameter components. This figure is reproduced with permission from [29].

\section{B. Results and Discussion}

\section{Physical Properties}

\subsection{Density and Cell Structure}

Density results of all foam compositions are reported in Table 8. Sufficiently low-density foams were seen for all foams with values ranging from 0.013 to $0.027 \mathrm{~g} \cdot \mathrm{cm}^{-3}$. These values suggest an average porosity $\left[\left(\rho_{\text {neat }}-\rho_{\text {porous }}\right) / \rho_{\text {neat }}\right]$ of $\sim 98 \%$ 
and a high average theoretical volume expansibility $\left(=\rho_{\text {neat }} / \rho_{\text {porous }}\right)$ of $\sim 60$ times; here the average $\rho_{\text {neat }} \sim 1.1 \mathrm{~g} \cdot \mathrm{cm}^{-3}$ is the neat polymer density and $\rho_{\text {porous }}$ is the foam density. The small standard deviation of $0.001-0.006 \mathrm{~g} \cdot \mathrm{cm}^{-3}$ (Table 8 ) in the density measurements indicates a fairly uniform structure of the foams.

Table 8: Summary of the key physical properties of the different foam compositions. This table is reproduced with permission from [29].

\begin{tabular}{|c|c|c|c|c|c|c|c|c|}
\hline $\begin{array}{l}\text { Sample } \\
\text { ID }\end{array}$ & $\begin{array}{l}\text { Density } \\
\left(\mathrm{g} \cdot \mathrm{cm}^{-3}\right)\end{array}$ & $\begin{array}{c}T_{g} \\
\left({ }^{\circ} \mathbf{C}\right)\end{array}$ & $\begin{array}{c}G_{\text {glassy }}^{\prime} \text { at } \\
\mathrm{T}_{\delta \mathrm{s}^{-20}}{ }^{\circ} \mathrm{C} \\
(\mathbf{k P a})\end{array}$ & $\begin{array}{c}G_{r u b b e r y}^{\prime} \text { at } \\
\mathrm{T}_{\delta s}+20^{\circ} \mathrm{C} \\
(\mathbf{k P a})\end{array}$ & $\begin{array}{l}T_{\text {onset }} \\
\left({ }^{\circ} \mathbf{C}\right)\end{array}$ & $\begin{array}{c}\boldsymbol{T}_{\delta s} \\
\left({ }^{\circ} \mathbf{C}\right)\end{array}$ & $\begin{array}{c}\Delta T \\
\left({ }^{\circ} \mathbf{C}\right)\end{array}$ & $\begin{array}{l}\text { Tan } \delta \\
\text { (Peak } \\
\text { value) }\end{array}$ \\
\hline 100TM & $.014 \pm .001$ & 75 & $108 \pm 7$ & $2 \pm 1$ & $58 \pm 1$ & $80 \pm 1$ & $40 \pm 0$ & $1.04 \pm .02$ \\
\hline 80TM & $.013 \pm .002$ & 71 & $103 \pm 6$ & $3 \pm 1$ & $57 \pm 2$ & $77 \pm 1$ & $42 \pm 1$ & $1.05 \pm .06$ \\
\hline 60TM & $.025 \pm .001$ & 69 & $311 \pm 28$ & $13 \pm 2$ & $53 \pm 1$ & $70 \pm 3$ & $37 \pm 1$ & $0.91 \pm .01$ \\
\hline 40TM & $.017 \pm .001$ & 68 & $90 \pm 3$ & $3 \pm 2$ & $57 \pm 3$ & $77 \pm 1$ & $35 \pm 3$ & $1.02 \pm .02$ \\
\hline 20TM & $.027 \pm .006$ & 65 & $158 \pm 24$ & $6 \pm 2$ & $53 \pm 3$ & $66 \pm 3$ & $27 \pm 4$ & $0.89 \pm .04$ \\
\hline OTM & $.018 \pm .004$ & 63 & $155 \pm 46$ & $6 \pm 1$ & $51 \pm 2$ & $64 \pm 1$ & $30 \pm 4$ & $0.71 \pm .04$ \\
\hline
\end{tabular}

Optical microscopy was used to determine cell structure, with typical structures shown in Figure 24. Observations include uniform closed or mixed closed to open cell structure for a given composition, with thin residual membranes between struts as previously reported for similar materials [30]. At the same time cell sizes varied for the different compositions. A significant factor contributing to the size variation is believed 
to be the variation in the foaming solution viscosity ( 2 to $60 \mathrm{~Pa} \cdot \mathrm{s})$, which was not a controlled variable across different compositions. It has been observed that the cell sizes of the foams can be controlled by controlling the viscosity of the foaming solution; the sizes are generally seen to decrease with increase in the foaming solution viscosity.

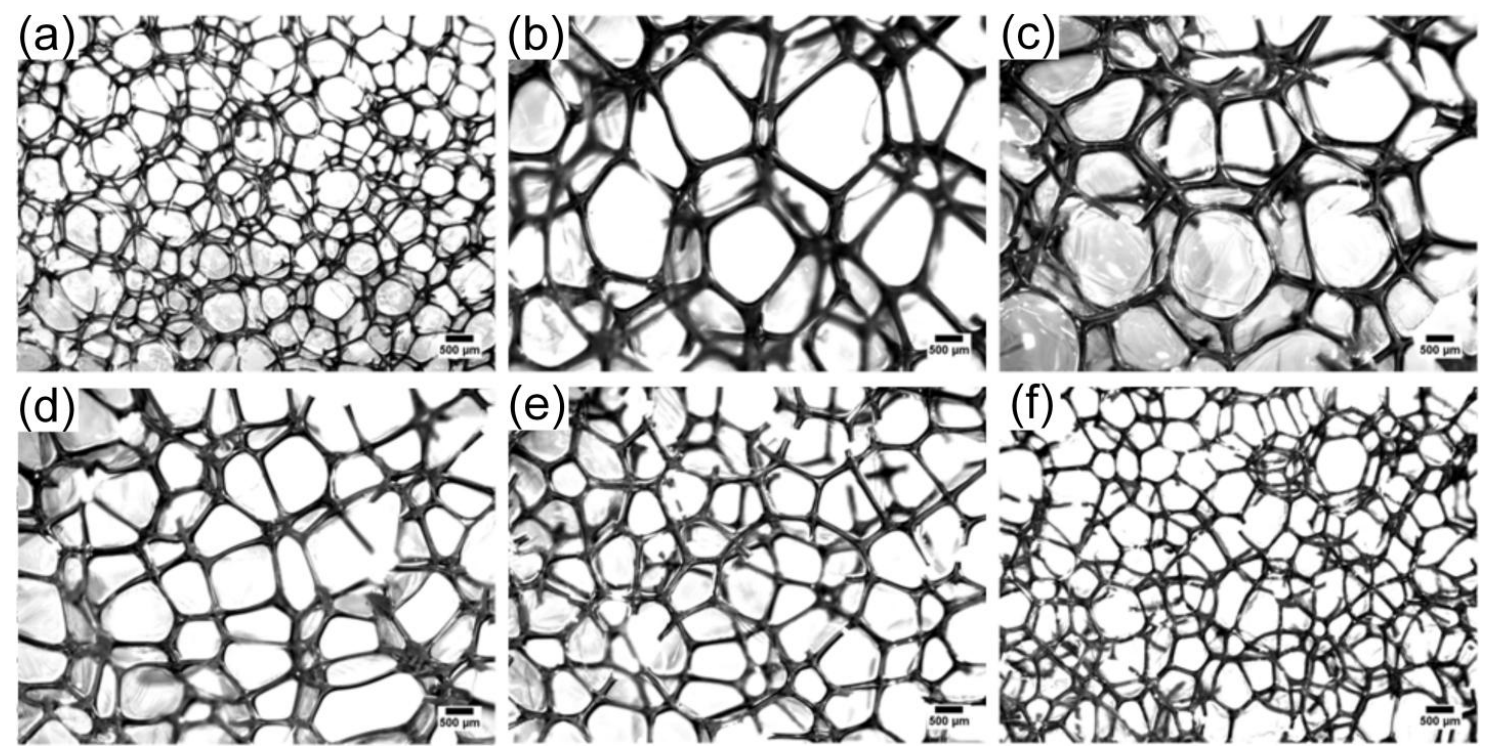

Figure 24: Cell structure of the foams as obtained from optical microscopy. Images (a), (b), (c), (d), (e) and (f) represent 0TM, 20TM, 40TM, 60 TM, 80TM and 100TM compositions, respectively (scale bar is $500 \mu$ ). This figure is reproduced with permission from [29].

It is important to note here that the primary design objectives for the proposed application (low-density and uniform cell structure), as described in Singhal et al. [30], were successfully met for all compositions. Hence, properties specific to the embolic biomedical applications of these materials, such as high crimping efficiency of the device and a large volume expansion on actuation, may be realized. Also, they have a 
highly crosslinked network architecture, which is expected to provide optimal shape memory properties as described by Singhal et al. [30].

\subsection{DSC}

DSC results of the foams are shown in Table 8 and Figure 25 . The foams show a single $T_{g}$ with values varying within a narrow range of $63-75{ }^{\circ} \mathrm{C}$. Lack of any crystallization or melting region in the DSC thermogram confirms the amorphous nature of the polymer network. An increase in the TMHDI content was seen to increase the effective $T_{g}$ of the foams. This is expected as the methyl groups of TMHDI can increase the energy required by the molecule to rotate about the backbone bonds.

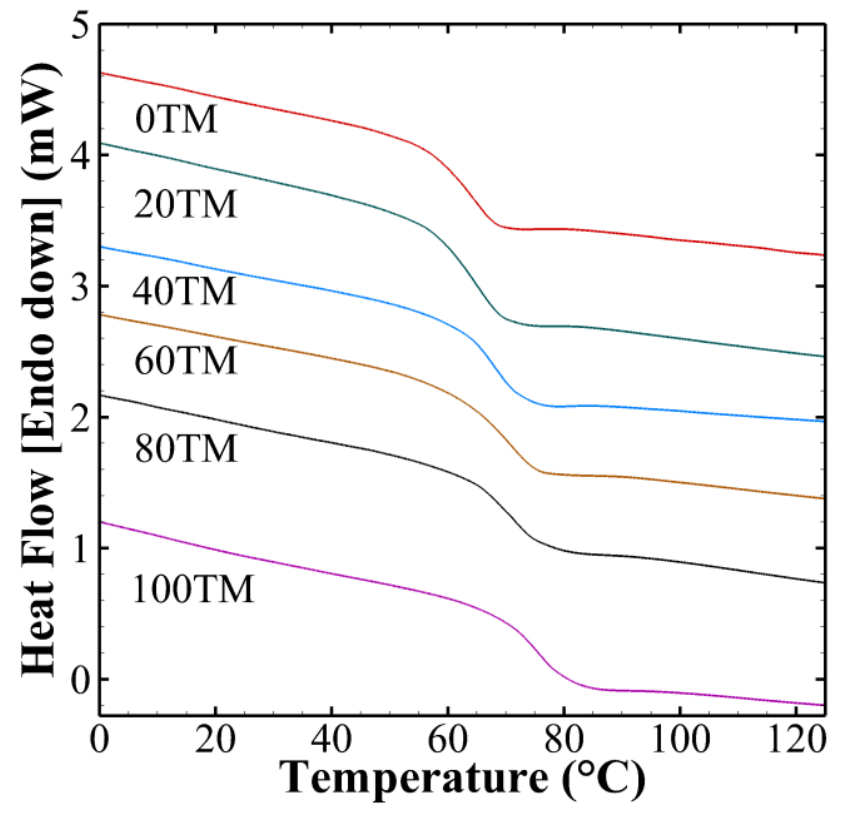

Figure 25: DSC curves of the different foam compositions. Single sharp glass transitions were seen for all compositions within a small range of $63-75^{\circ} \mathrm{C}$. This figure is reproduced with permission from [29]. 


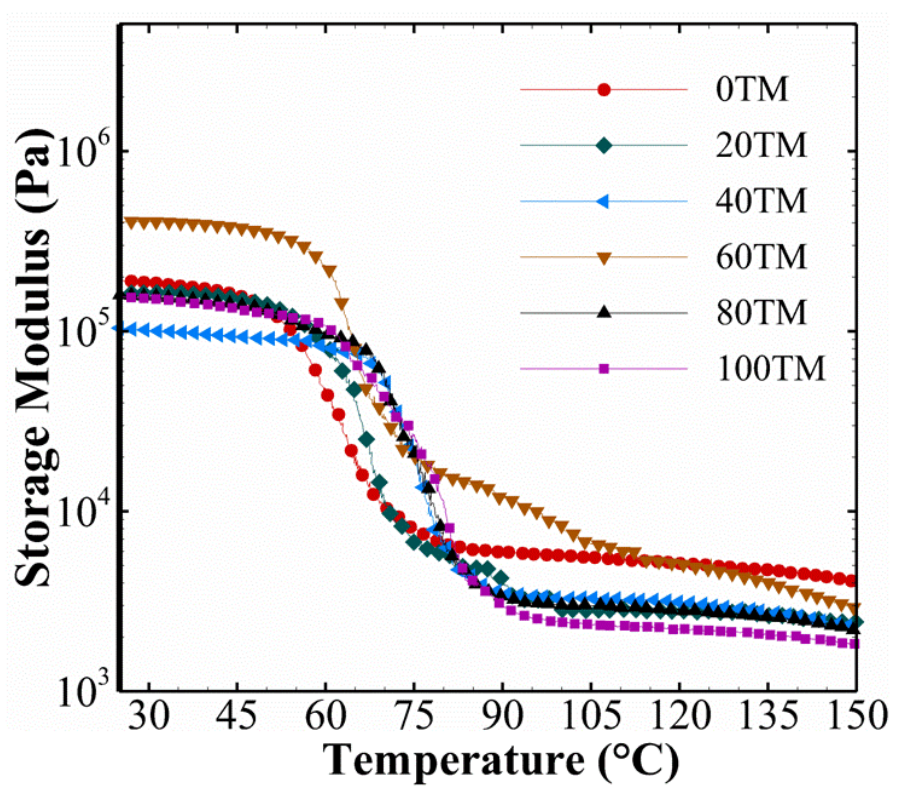

Figure 26: DMTA curves of the different foam compositions. Single sharp glass transitions were seen for all compositions within a small range of 64-80 ${ }^{\circ} \mathrm{C}$. This figure is reproduced with permission from [29].

\subsection{DMTA}

DMTA results of the foams are shown in Table 8 and Figure 26 . A variation in the glassy modulus ( $G_{\text {glassy }}^{\prime}$ ) is recorded from 311 to $90 \mathrm{kPa}$ across different compositions. To explain this relatively large range, variation in the densities of these compositions was accounted for in the $G_{\text {glassy }}^{\prime}$ estimations. The reference of 0TM was used and the value of $G_{\text {glassy }}^{\prime}$ at $0.018 \mathrm{~g} \cdot \mathrm{cm}^{-3}$ was extrapolated using $G_{1}=G_{2} \cdot\left(\rho_{1} / \rho_{2}\right)^{2}$ as per Gibson et al. [111], where $G$ is the modulus, $\rho$ is the material density, and subscripts 1 and 2 denote corrected and measured values, respectively. Average glassy moduli of 70, 101, 161, 197, and $179 \mathrm{kPa}$ were thus calculated for 20TM, 40TM, 60TM, 80TM, and 100TM foams, respectively. While the values of other compositions are similar when extrapolated to the same density, 20TM and 40TM foams have relatively lower 
moduli $(70-101 \mathrm{kPa})$. This may be due to larger cell sizes of these foams as seen in Figure 24. Surface effects have been shown to become more prominent as the dimensions of the tested sample approach those of the cell size, and a reduction in foam modulus and strength is often noticed in such cases $[159,160]$. The DMA measured $T_{g}$ of all the materials is within $\sim 16{ }^{\circ} \mathrm{C}$, leading to a significant overlap in the storage modulus versus temperature curves (Figure 26). The onset of transition from the glassy to the rubbery state is approximately $55 \pm 3{ }^{\circ} \mathrm{C}$, and $T_{g}$ (estimated as the temperature at the peak value of the $\tan \delta$ curve) is $72 \pm 7^{\circ} \mathrm{C}$ across all compositions (Table 8). A small but positive increase in the $T_{g}$ with increase in the TMHDI content is seen. This follows the trend observed in the DSC measurements (Figure 25). Also, a single thermal transition from the glassy to the rubbery state, with a transition range of $\sim 35 \pm 6{ }^{\circ} \mathrm{C}$ was recorded for all compositions. The presence of a single thermal transition with a constant rubbery modulus confirms an amorphous and crosslinked polymer network structure of the foams.

\subsection{Fourier Transform Infrared Spectroscopy (FTIR)}

The FTIR spectra of the foams of different compositions are shown in Figure 27. The spectra of the compositions are qualitatively similar, and the typical features of the spectra reported earlier for related compositions [30] are evident for all compositions. These include the hydrogen-bonded urethane peak at $\sim 1695 \mathrm{~cm}^{-1}$ and a urea shoulder at $\sim 1653 \mathrm{~cm}^{-1}$. The position of these peaks, however, is shifted ( by $\sim 3-6 \mathrm{~cm}^{-1}$ ) compared with previously reported foams [30]. This may be related to the difference in the 
physical condition of the tested samples [161]. The compression of the foams to a flat sheet for performing attenuated total reflectance (ATR) FTIR done in the previous study [30] may have enhanced hydrogen bonding of urethane/urea groups leading to a shift of urethane and urea absorption to slightly lower values.

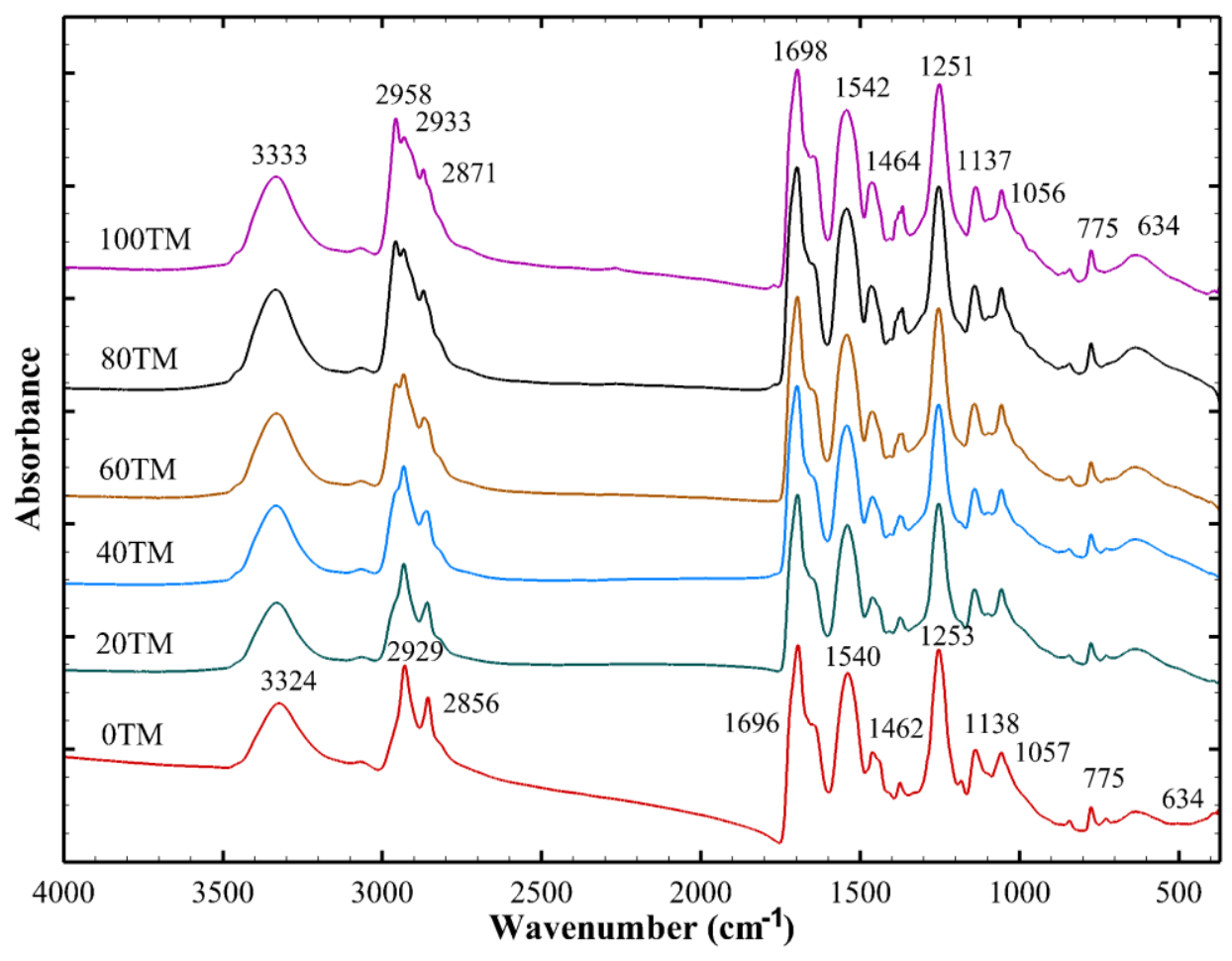

Figure 27: FTIR curves of the different foam compositions. Changes in the C-H vibrations of the $-\mathrm{CH}_{3}$ groups of TMHDI are evident in the $2800-3000 \mathrm{~cm}^{-1}, 1430$ $1490 \mathrm{~cm}^{-1}$, and $1350-1400 \mathrm{~cm}^{-1}$ ranges. This figure is reproduced with permission from [29].

On the other hand, the effect of TMHDI can be readily observed across the different compositions. The differences in the $\mathrm{C}-\mathrm{H}$ vibrations due to the inclusion of methyl side groups of TMHDI can be seen in the 2800-3000, 1430-1490, and 
$1350-1400 \mathrm{~cm}^{-1}$ wavenumber ranges. The peaks at 2958 and $2871 \mathrm{~cm}^{-1}$ in the 100TM foam correspond to asymmetrical and symmetrical $\mathrm{C}-\mathrm{H}$ stretching vibrations of the $-\mathrm{CH}_{3}$ groups, respectively [162]. The peaks at 1464 and $1370 \mathrm{~cm}^{-1}$ in the 100TM foam correspond to the asymmetrical and symmetrical $\mathrm{C}-\mathrm{H}$ deformation vibrations of the $-\mathrm{CH}_{3}$ groups, respectively [162]. These peaks are seen to increase in intensity due to an increase in the $\mathrm{C}-\mathrm{H}$ vibration as the amount of TMHDI is increased.

\section{Characterization of Foam Actuation and Actuation-related Properties}

\subsection{Contact Angle}

The contact angle was measured on neat/unfoamed polymer samples as these measurements on foam samples were difficult, and subject to high variability due to the porous nature of the foam surface. The measurement results of the corresponding compositions are shown in Table $\mathbf{9}$, and Figure 28 illustrates the change in contact angle as we progress from the 0TM to the 100TM composition. A decrease in the contact angle from $78^{\circ}$ to $62^{\circ}$ is seen as the TMHDI content is decreased in the composition. This result supports the hypothesis that the introduction of methyl groups in the polymer network via use of TMHDI increases the hydrophobicity of the material. Although the absolute values of contact angle for the corresponding foam formulations can be different from those obtained from the neat samples due to the presence of urea groups from the use of water as a chemical blowing agent in the foams, they are expected to follow a similar trend with the variation in the foam composition. 
(a)

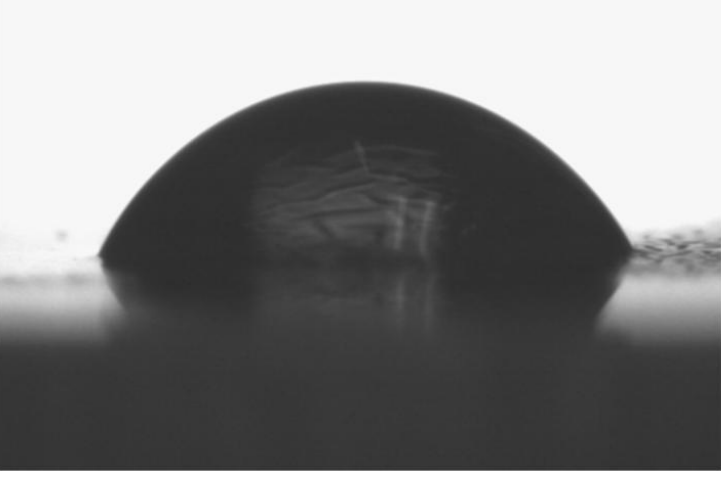

(b)

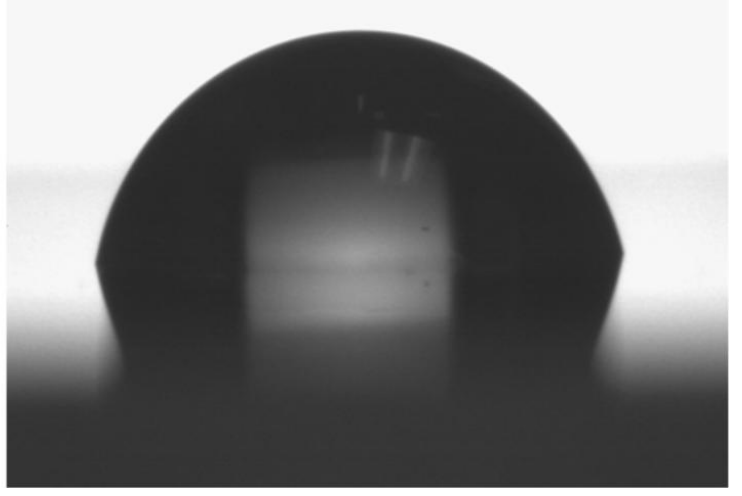

Figure 28: Contact angle images of (a) 0TM and (b) 100TM neat/unfoamed polymer compositions. An increase in the contact angle is noticed with an increase in the TMHDI content of the polymer. This figure is reproduced with permission from [29].

Table 9: Summary of the results related to the actuation behavior of the foams. This table is reproduced with permission from [29].

\begin{tabular}{|c|c|c|c|c|}
\hline Sample ID & $\begin{array}{c}\text { Equilibrium } \\
\text { moisture uptake } \\
\text { (wt.\%) }\end{array}$ & $\begin{array}{l}\text { Contact angle } \\
\text { (Degrees) }\end{array}$ & $\begin{array}{c}\text { Equilibrium } T_{g}{ }^{1} \\
\left({ }^{\circ} \mathrm{C}\right)\end{array}$ & $\begin{array}{l}\text { Estimated Working } \\
\text { Time }^{2} \text { (minutes) }\end{array}$ \\
\hline 100TM & $2.3 \pm 1.0$ & $78.08 \pm 2.58$ & $39.1 \pm 0.3$ & 10 \\
\hline 80TM & $4.3 \pm 0.7$ & $74.73 \pm 2.51$ & $30.6 \pm 1.3$ & 5 \\
\hline 60TM & $4.4 \pm 0.9$ & $74.06 \pm 0.83$ & $26.4 \pm 0.4$ & 4 \\
\hline 40TM & $6.0 \pm 0.8$ & $70.62 \pm 3.51$ & $21.1 \pm 1.7$ & 3 \\
\hline 20TM & $5.0 \pm 0.8$ & $66.26 \pm 4.97$ & $15.0 \pm 0.7$ & $<3$ \\
\hline OTM & $8.1 \pm 1.9$ & $62.55 \pm 2.86$ & $12.2 \pm 0.6$ & $<1$ \\
\hline
\end{tabular}

\footnotetext{
${ }^{1}$ Measured after 2 hours of submersion in water.
}

${ }^{2}$ Defined here as the time until $\sim 20 \%$ of the initial diameter is recovered in $37{ }^{\circ} \mathrm{C}$ water (representative of, but not necessarily equivalent to, the working time for an actual catheter-delivered device). 


\subsection{Equilibrium Moisture Uptake}

Equilibrium water absorption values are reported in Table 9. Water uptake decreases from $\sim 8$ to $\sim 2 \%$ as the TMHDI content is increased in the foam composition. This result is in agreement with the contact angle results and is a direct measure of the increase in hydrophobicity of the foams with increasing TMHDI content. The slight discrepancy in the trend may be due to the different cell sizes (different surface areas) of the foam samples.

\subsection{Depression in $T_{g}$ with Exposure to Moisture}

The results of depression in the foam $T_{g}$ on its exposure to water are shown in Table 9 and Figure 29. All foam compositions reach their equilibrium plasticized $T_{g}$ value within 5 minutes of exposure to water. Kinetically, the depression in $T_{g}$ is fast enough that it may be considered independent of the TMHDI composition within the tested time scale. The extent of depression of $T_{g}$, however, progressively decreases as the TMHDI content is increased, with the equilibrium $T_{g}$ value going from $\sim 12{ }^{\circ} \mathrm{C}$ for the 0TM foams to $\sim 40{ }^{\circ} \mathrm{C}$ for the $100 \mathrm{TM}$ foams. Consequently, the extent of plasticization due to moisture is found to be lower (higher equilibrium $T_{g} \sim 40{ }^{\circ} \mathrm{C}$ ) for the most hydrophobic polymer composition. 


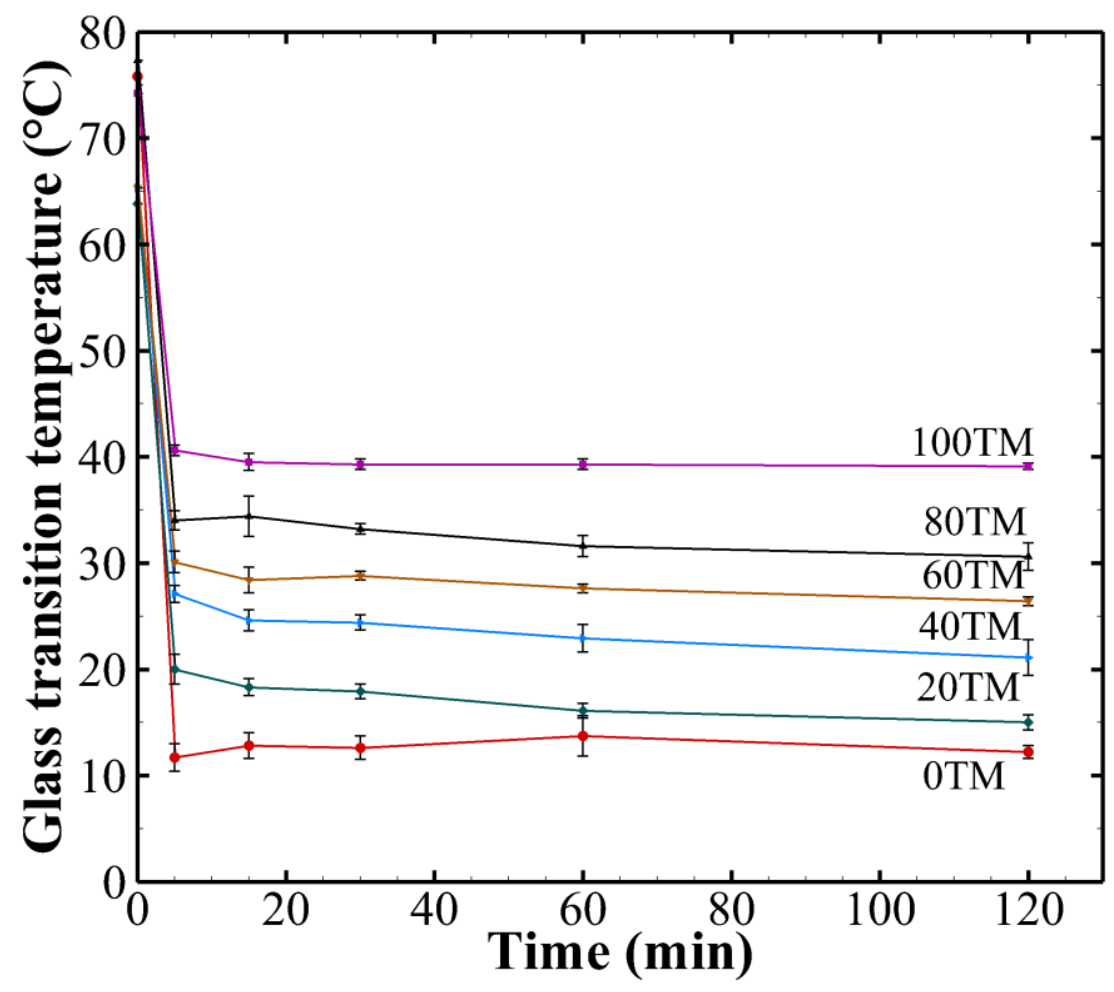

Figure 29: Change in the glass transition temperature $\left(T_{g}\right)$ of the foam samples with respect to time submerged in water at $37^{\circ} \mathrm{C}$. The equilibrium $T_{g}$ value is achieved within 5 minutes for all compositions. An increase in the equilibrium $T_{g}$ of the foams is noticed. This figure is reproduced with permission from [29].

\subsection{Rate of Expansion of Foam in Fluid Media at $37^{\circ} \mathrm{C}$}

Results of the rate of expansion of foam samples in water are shown in Table 9 and Figure 30 and Figure 31. The rate of actuation decreases with increase in the amount of TMHDI in the foam formulation. The 0TM foam samples show no delay in actuation and achieve complete actuation within 2 minutes of exposure to water. Conversely, 100TM foam samples maintain their secondary compressed shape for $\sim 10$ minutes under water, and do not recover completely to their primary shape even after 1 hour. The error bars in Figure 31 are relatively large because the maximum diameter of 
the expanding foams was used to generate the plot. As is evident in Figure 30 , even though the average trend of actuation is the same within a given composition, the maximum diameter is not uniform across the entire length of the sample and varies across different samples of the same composition.

(a)

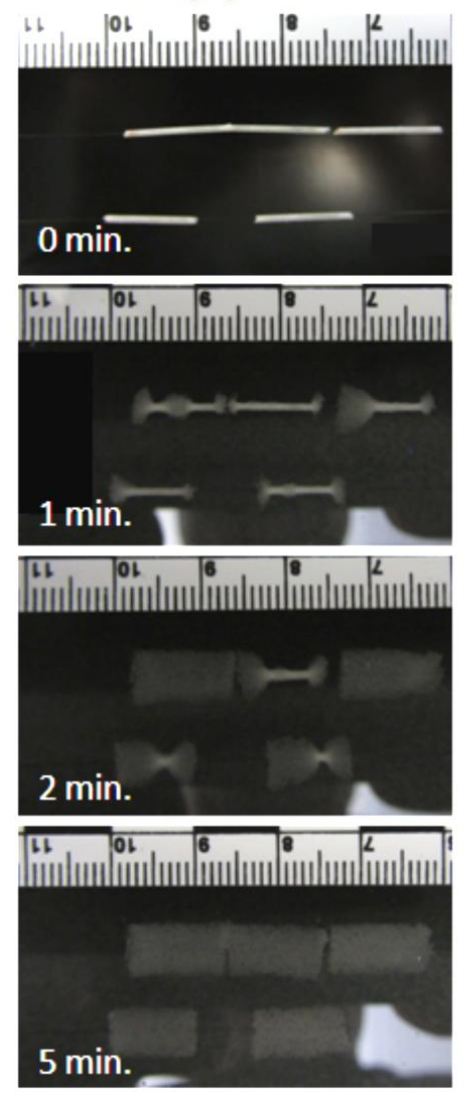

(b)

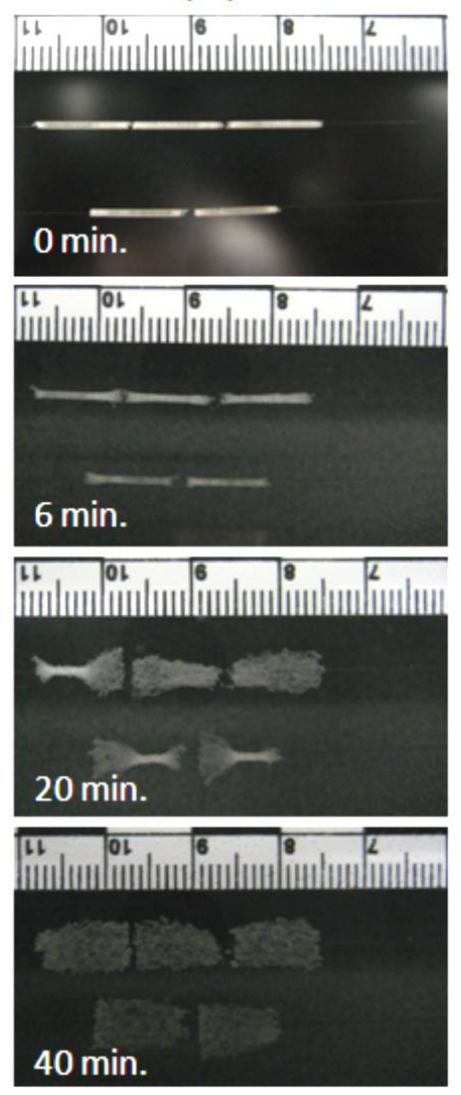

(c)
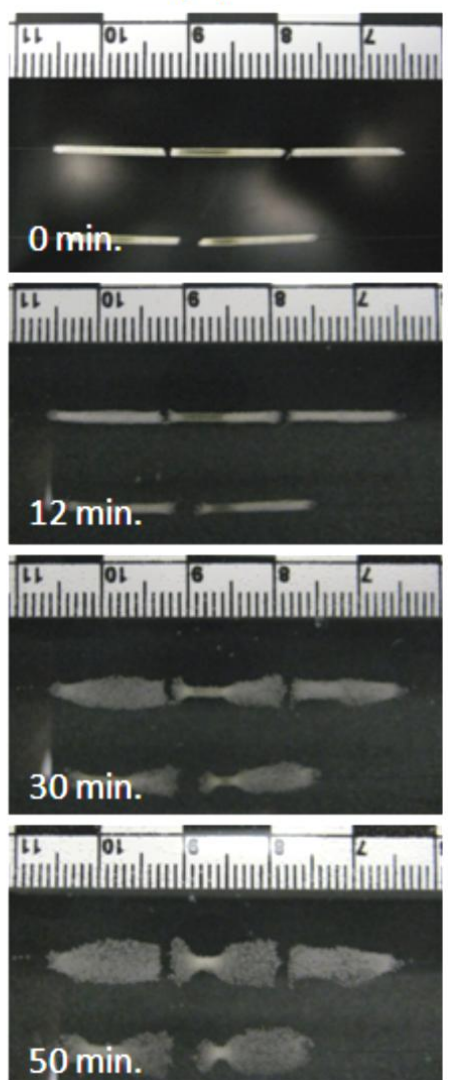

Figure 30: Rate of actuation of foam samples of (a) 0TM, (b) 80TM and (c) 100TM compositions. While the OTM foams with only HDI in their composition actuated completely within 5 minutes of exposure to water at $37^{\circ} \mathrm{C}$, the $100 \mathrm{TM}$ foams did not complete their actuation even at 50 minutes. This figure is reproduced with permission from [29]. 


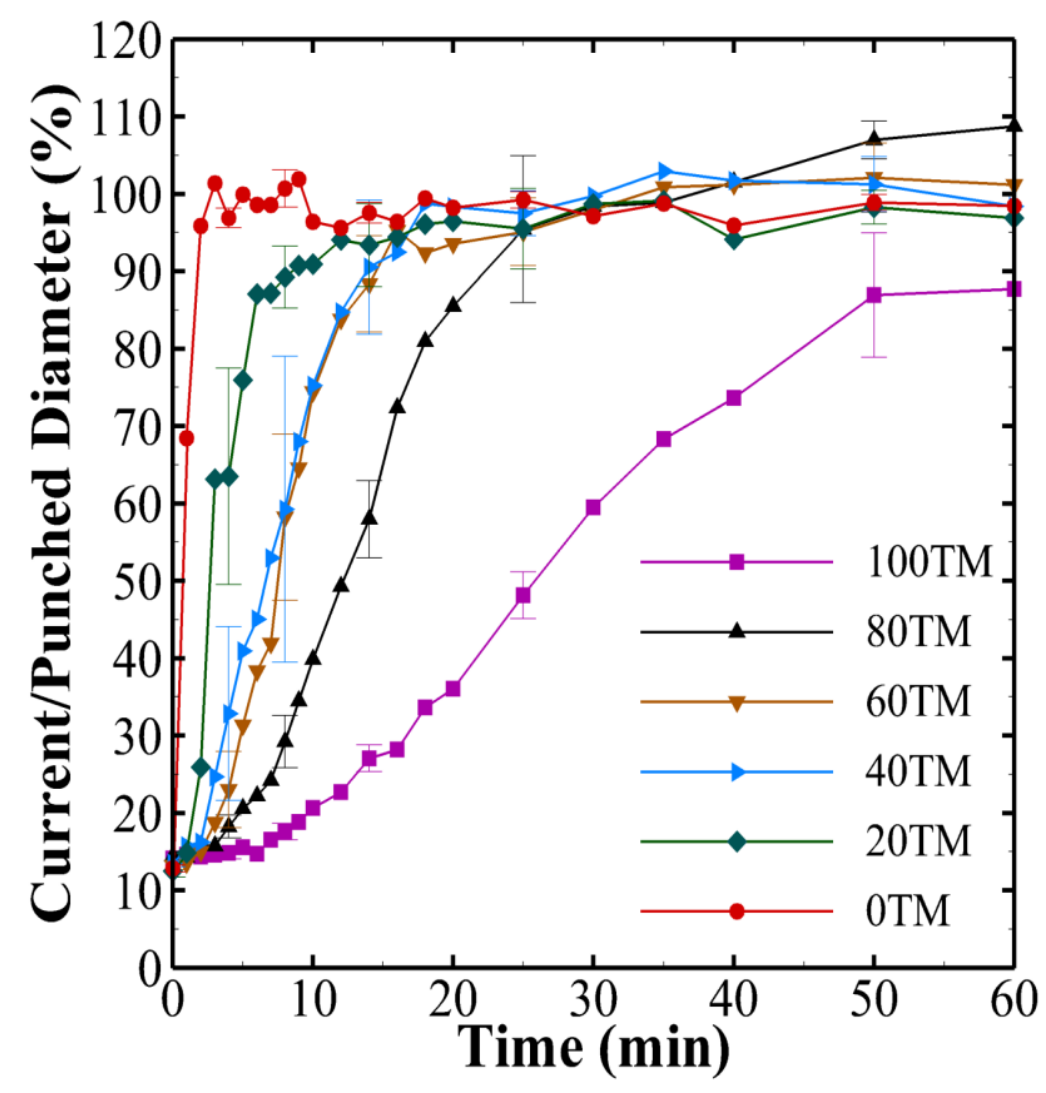

Figure 31: Ratio of the maximum diameter to the initial/punched diameter as a function of time in $37^{\circ} \mathrm{C}$ water for all foam compositions. Only every fourth error bar is marked for clarity. A decrease in the rate of expansion of the SMP foam is seen with an increase in the TMHDI content. This figure is reproduced with permission from [29].

The foam actuation rate depends on the depressed $T_{g}$ in the water and the temperature of the water. As the depressed $T_{g}$ of the $100 \mathrm{TM}$ foam is $\sim 40{ }^{\circ} \mathrm{C}$ (i.e., slightly above the water temperature), it does not actuate as readily as the 0TM foam whose depressed $T_{g}$ of $\sim 12{ }^{\circ} \mathrm{C}$ is well below the water temperature $\left(37^{\circ} \mathrm{C}\right)$. It should be noted here that although these tests are conducted in water, the foams are expected to have a similar trend of actuation behavior in physiological media due to its high water content. 
Also, these results are reported for $6 \mathrm{~mm}$ diameter cylindrical samples. Although the trends across different compositions are expected to be the same, the absolute actuation time may increase with an increase in the sample size.

\subsection{Theoretical Solubility Parameter Analysis}

Hoy's solubility parameters calculated for different polymer compositions are reported in Table 10. The objective of doing these calculations was to develop an understanding of the changes in the solvent (water) and polymer interactions with the variation in the polymer formulation. It is well known that the difference between the solubility parameter $\left(\delta_{t}\right)$ value of a crosslinked polymer and that of the solvent is directly related to the degree of polymer swelling in that solvent [158]. The calculated net $\delta_{t}$ of 0TM and 100TM compositions are 23.0 and $21.3 \mathrm{~J}^{1 / 2} \cdot \mathrm{cm}^{-3 / 2}$ respectively, resulting in a higher difference in the polymer and water $\delta_{t}$ values for the 100TM composition $\left(\delta_{t \text { water }}=48 \mathrm{~J}^{1 / 2} \cdot \mathrm{cm}^{-3 / 2}\right)$. This suggests that the 0TM foams would undergo a higher equilibrium swelling in water compared with the 100TM foams, due to their relatively favorable chemical environment in water. The higher extent of equilibrium swelling is expected to influence the depression in $T_{g}$, and therefore the rate of actuation of the foam devices in water. 
Table 10: Hoy's solubility parameters calculated for the different foam compositions. This table is reproduced with permission from [29].

\begin{tabular}{|c|c|c|c|c|}
\hline Sample ID & $\delta_{d}\left(\mathbf{J}^{1 / 2} \cdot \mathbf{c m}^{-3 / 2}\right)$ & $\delta_{p}\left(\mathbf{J}^{1 / 2} \cdot \mathbf{c m}^{-3 / 2}\right)$ & $\delta_{h}\left(\mathbf{J}^{1 / 2} \cdot \mathbf{c m}^{-3 / 2}\right)$ & $\delta_{t}\left(\mathbf{J}^{1 / 2} \cdot \mathbf{c m}^{-3 / 2}\right)$ \\
\hline 100TM & 16.7 & 10.6 & 7.8 & 21.3 \\
\hline 80TM & 16.6 & 10.8 & 8.5 & 21.6 \\
\hline 60TM & 16.7 & 11.1 & 8.8 & 21.9 \\
\hline 40TM & 16.7 & 11.3 & 9.3 & 22.2 \\
\hline 20TM & 16.7 & 11.6 & 9.8 & 22.6 \\
\hline 0TM & 16.7 & 11.9 & 10.4 & 23.0 \\
\hline
\end{tabular}

To better understand the foam actuation mechanisms, we now discuss the effect of the polymer chemical structure on its interaction with water. In previous studies, it has been found that the water molecules weaken the hydrogen bonding between $\mathrm{N}-\mathrm{H}$ and $\mathrm{C}=\mathrm{O}$ groups and plasticize the polymer network, causing a depression in the $T_{g}$ of the polyurethane SMP materials in high-humidity/water environments [55, 137, 144]. Water molecules have a small size and a high hydrogen bonding potential. Hence, when exposed to a polyurethane SMP, they can disrupt the hydrogen bonds between $\mathrm{N}-\mathrm{H}$ and $\mathrm{C}=\mathrm{O}$ groups of the urethane linkages of adjacent chains, and can then be inserted between these groups via mutual hydrogen bonding. Given that the hydrogen bonding is largely dynamic in nature, insertion of water molecules between adjacent chains essentially works as a lubricant, thereby increasing the mobility of the chains, that is, plasticizing the polymer [163]. By this analogy, the change in the degree of depression of $T_{g}$ seen for the series of compositions here should be a function of the extent of interaction of water molecules with the urethane hydrogen bonding in the polymer. Indeed, as demonstrated by the water absorption studies and the theoretical 
determination of solubility parameters, the amount of equilibrium water absorbed depends on the chemical environment of the polymer. Due to higher differences in the cohesive energy densities, it is relatively energetically unfavorable for the water molecules to coexist with the polymer and undergo hydrogen bonding in the presence of higher amounts of methyl groups. Hence, a lower amount of water is absorbed at equilibrium by materials with a higher TMHDI content. With fewer water molecules between adjacent chains, that is, lower amount of equilibrium absorption of water or swelling, the increase in polymer chain mobility is lower, leading to a lower depression in $\mathrm{T} \mathrm{g}$ in the 100TM composition. Conversely, a higher water absorption or swelling leads to a higher increase in mobility and hence a higher depression in $T_{g}$ in the 0TM composition.

The primary goal of this study was to achieve improved control over the rate of actuation of SMP foam to enhance its use in a device for aneurysm treatment. The SMP foam devices are proposed to be delivered to the aneurysm site via a catheterization process while they are in their completely compressed rod-like secondary shape. As the device approaches the aneurysm site, the clinician must carefully position the device within the aneurysm so that it does not protrude into the parent artery. Ideally, the device should not start expanding until these steps of delivery and positioning are completed. However, after these steps are complete, the device should expand at a relatively fast rate to fill the aneurysm. In this regard, a concern with moisture sensitive SMP devices is that the guiding catheters and microcatheters used in surgical processes are routinely flushed with saline solution during the device delivery. This may cause a 
moisture-sensitive material to potentially begin expanding inside the catheter during the delivery process. It is therefore desirable to reduce the moisture sensitivity of the device such that it is able to withstand body temperature fluid environments without actuation during the entire duration of its delivery and positioning. This duration can range from 1 minute to up to 5-10 minutes, depending on the patient vasculature and the location of the aneurysm [164].

This work demonstrated that increasing the hydrophobicity of the monomers in the foam synthesis can reduce its rate of actuation. This would allow the device to initially maintain its near-compressed shape for a longer period in water/physiological environments, and later still actuate to its primary shape in a passive/active manner to fill the aneurysm. The passive actuation of a device on exposure to water/physiological media may allow the usage of a simpler deployment device without a heating capability. However, it presents a practical tradeoff in the rate of actuation versus the working time of the device (time for which the device maintains its near-compressed secondary shape while being exposed to water/physiological media). A lower rate of actuation gives a longer device working time (as in 100 TM foams, Figure 30), but complete actuation also takes a relatively long time, which may not be preferable in all cases.

A possible strategy to mitigate the tradeoff between the rate of actuation and the working time is to use a material that has high hydrophobicity to facilitate delivery and positioning of the device, and thereafter actuate it actively by applying thermal energy. The actuation temperature of these materials can be changed by varying the ratio of the hydroxyl components HPED and TEA, as reported in Singhal et al. [30], so that they 
may be actuated with a relatively small amount of thermal energy to minimize the potential for thermal tissue damage. Employing active actuation of the device while utilizing the above polymer synthesis toolbox is thus expected to give a higher level of predictability and control on the actuation of the SMP foam devices during their deployment for in vivo embolic or regenerative applications.

\section{Conclusion}

SMPs have been shown to actuate below their dry glass transition temperatures in the presence of moisture due to plasticization. This behavior has been proposed as a self-actuating mechanism of SMPs in water/physiological media. However, control over the SMP actuation rate, a critical factor for in vivo transcatheter device delivery applications, has not been previously reported. Here, a series of polyurethane SMPs with systematically varied hydrophobicity is described that permits control of the time for their complete shape recovery in water from under 2 minutes to more than 24 hours. This control over the SMP actuation rate can potentially provide significant improvement in their delivery under conditions, which may expose them to highmoisture environments prior to actuation. 


\section{CHAPTER IV}

\section{SYNTHESIS AND POST-SYNTHESIS MODIFICATIONS FOR REDUCED DENSITIES AND COMPLETE RETICULATION OF FOAMS}

As the title suggests, this chapter aims to discuss foam fabrication and processing modifications in order to achieve still lower densities $\left(<0.015 \mathrm{~g} \cdot \mathrm{cm}^{-3}\right)$, and complete removal of residual cell membranes from the foam cell surfaces. Both these developments were critical for the proposed application of aneurysm treatment with respect to achieving a higher embolic efficiency and control over material morphology.

Previous chapters demonstrated the gas blowing process as being highly conducive to the development of low density foams using polyurethane chemistry. However, we did not elaborate on the complexity of this method: it is a dynamic process where several factors such as raw materials $[165,166]$, polyol functionality $[167,168]$, isocyanate index [169], solution viscosity, blowing agent $[170,171]$, type and concentration of catalysts and surfactants [172-176], temperature of foaming [166, 177] and curing and mold size $[132,178]$, can play a significant role in the final physical properties of the foams. This chapter reports a broad range of formulative and process changes in foam fabrication, to achieve a new paradigm in low density SMP foams

$\left(\rho_{\text {porous }}<0.015-0.020 \mathrm{~g} \cdot \mathrm{cm}^{-3}\right)$, and discusses the mechanism of action of these improvements.

The other topic addressed in this chapter is modulating the morphology/structure of the foams. This is another challenging aspect because of the complex role of dynamic 
film and interfacial tensions in these systems during the rise of the foam [179]. The phenomenon involved in this process was summarized in by Scriven and Sterling in 1960 [180], and have since been studied in depth in multiple relevant foaming contexts [179, 181-183]. Briefly, the interfacial tensions, of the polymer-air surface during the foam rise, determine the extent of residual cell membranes via controlling the drainage of the polymer into the foam struts ([179], Appendix I). As a result, blown foams typically show a combination of open, closed, and partially closed cells [166]. However, with the growing utility in these foams in the medical industry, it is important to effectively control the removal of residual cell membranes in these materials in order to prevent any adverse possibility of dislodging of the membrane fragments and their downstream embolization, or inadequate permeation of blood/physiological fluid post implantation.

Efforts towards removal of residual cell membranes, also known as reticulation, of foams have long been under investigation. One of the earliest methods was based on leaching of the polyurethane foams with different solutions. Powers et al. [154] used one or more of sodium hydroxide, cellulose acetate, acetone, water, viscose solution and ethyl alcohol in varying concentrations for the foam reticulation. Another leaching technique was described by R. A. Volz, where he demonstrated removal of thin membranes via hydrolytic action of water in presence of a hydrolytic catalyst [156]. Also around this time, several other patents on this topic came out that involved, for e.g., leaching of foams with varying concentrations of sodium hydroxide [153]. Methods other than leaching were also investigated; Geen et al. showed that destruction of thin 
membranes is possible by volatization, or heat based destruction, via a light pulse of controlled intensity. Degree of reticulation could be determined precisely in this method [151]. Also, it was shown that efficient removal of diaphanous membranes of foams can be achieved via an explosive mixture of a combustible gas and oxygen [152]. Stark et al. suggested use of mechanical force, via high velocity air jets and pressure vessels for removal of foam cell membranes, in combination with a chemical weakening agent like methylene chloride [155]. Some variations of these methods have been used by our

group previously, for e.g. hydrochloric acid based etching and cleaning of these foams (Chapter III), and they were determined to be helpful in cleaning and getting rid of the residual mobile species in the foams. However, they were mostly ineffective with respect to achieving reticulation in the ultra low density foams. This may be attributed to the relatively high modulus of foams from the highly covalently crosslinked polymer structure, as well as the fragile ultra low density foam morphology.

Here a state-of-the-art technique of plasma reticulation was employed to achieve complete reticulation of the foams. Results of fabrication of foams with the modified blowing process, and their characterization are reported. Also, the effect of plasma reticulation process on the cell structure, density, and thermomechanical properties of the foams is reported and discussed.

\section{A. Materials and Methods}

The foams were fabricated using the gas blowing process, post-processed for membrane removal, and finally characterized for critical physico-mechanical properties. 


\section{Foam Synthesis}

HPED (99\%, Sigma Aldrich Inc.), TEA (98\%, Alfa Aesar Inc.), TMHDI (TCI America Inc.), Isophorone Diisocyanate (IPDI, TCI America Inc.), and DI water (Millipore water purifier system, Millipore Inc., $>17 \mathrm{M} \Omega \cdot \mathrm{cm}$ purity), were used as received. IPDI was incorporated to increase the hydrophobicity of the foams beyond what was achieved from TMHDI, based on the results of Chapter III [29] (Figure 32). Foams were synthesized according to the amounts indicated in Table 11. The three step method reported previously in Chapters II and III [29, 30] was used, with some modifications as specified below:

\subsection{Surfactants}

A new surfactant DC5943, instead of DC5179 and DCI990, (Air Products Corp.) was optimized and used for the synthesis process.

\subsection{Blowing Agent}

A higher amount of blowing agent, $7 \mathrm{ml}$ instead of $3 \mathrm{ml}$, (Enovate, Honeywell Inc.) was employed in the gas production during the foaming.

\subsection{Mold Size}

A 2.3 times larger mold was used for the foam rise to allow better expansion of the foam. 


\subsection{Process Change}

Vacuum was pulled during the foaming to assist in the foam rise and counter the effect of gravity and atmospheric pressure during foam rise.

Table 11: Summary of the foam composition.

\begin{tabular}{|c|c|c|c|c|c|c|c|c|c|}
\hline Component & TMHDI & IPDI & TEA & HPED & Water & DC5943 & BL-22 & T-131 & Enovate \\
\hline wt.\% & 63.45 & 3.38 & 5.69 & 17.01 & 2.32 & 7.12 & 0.25 & 0.78 & $\begin{array}{c}17.78 \\
(\mathrm{pph})\end{array}$ \\
\hline
\end{tabular}

The wt.\% value represents the net amount of the component in the final foamed polymer.

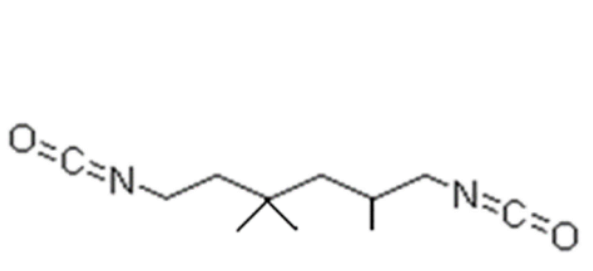

2,2,4 (and 2,4,4) Trimethyl 1,6

Diisocyanatohexane (TMHDI)

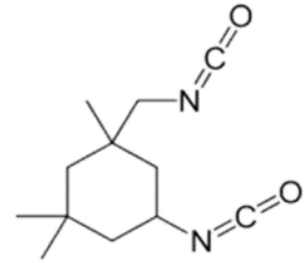

5-Isocyanato-1-(isocyanatomethyl)-1,3,3-trimethylcyclohexane or Isophorone Diisocyanate (IPDI)

Figure 32: Molecular structures of the two isocyanates, TMHDI and IPDI, used in the study.

Briefly, the $\mathrm{OH}$ and $\mathrm{NCO}$ premixes were mixed together in pre-determined quantities maintaining an isocyanate index of 104, and the physical blowing agent was added to the solution. The mixture was then poured into the molds and placed in the vacuum oven at $90{ }^{\circ} \mathrm{C}$. After an initial 2 minute of creaming and rise, vacuum was initiated in the oven. A slow rise in vacuum level $\left(\sim 2-4 \mathrm{~cm} \cdot \mathrm{Hg} \cdot \mathrm{min}^{-1}\right)$ was applied until $\sim 20 \mathrm{~cm} \cdot \mathrm{Hg}$. The vacuum was fixed at this level, and heat was turned off after 2 hours of 
cure. Foam was then allowed to cool down under vacuum for $\sim 12$ hours. Following this period, vacuum was released at a slow rate $\left(\sim 2-4 \mathrm{~cm} \cdot \mathrm{Hg} \cdot \mathrm{min}^{-1}\right)$, and foams were removed from the mold. Foams were allowed to further cure at room temperature for at least a week before testing.

\section{Foam Post-synthesis Processing}

For removal of residual cell membranes, the foams were subjected to plasma treatment. Foam samples $(\sim 1$ inch thick blocks) were placed in a plasma etching chamber (Plasma Technology Systems, Belmont, CA). An atmosphere of $\mathrm{O}_{2} / \mathrm{CF}_{4}$ gas mixture was purged in the chamber, and plasma was generated using a mild electrode. The samples were treated for 10-20 minutes at temperatures not exceeding $35^{\circ} \mathrm{C}$.

\section{Foam Characterization}

\subsection{Density and Cell Structure}

Core density of three foam samples was measured from the representative top and middle sections as per ASTM standard D-3574. For cell structure characterization, thin slices were cut from a representative section of foams with and without post-processing reticulation treatment. Images were captured in the brightfield mode on a Leica MZ8 microscope (Leica Microsystems Inc.) using RSImage Software (Roper Scientific Inc.). 


\subsection{DMTA}

For characterizing the mechanical properties of the foams as a function of temperature, a dynamic temperature ramp test was performed on an ARES-LS2 rheometer (TA Instruments Inc.) according to a previously described method [30]. A torsion rectangle test fixture was used on samples cut to approximately $45 \mathrm{~mm}$ long, 12 $\mathrm{mm}$ wide, and $6 \mathrm{~mm}$ thick with a gap distance of $25 \mathrm{~mm}$. The samples were prepared by embedding both ends in a polyurethane neat polymer to prevent damage and slippage of the foam sample in the metal grips. Dynamic temperature ramp tests were then run at a frequency of $1 \mathrm{~Hz}$ and constant heating rate of $1{ }^{\circ} \mathrm{C} \cdot \mathrm{min}^{-1}$ from $25^{\circ} \mathrm{C}$ to $150{ }^{\circ} \mathrm{C}$. An initial shear strain of $0.2 \%$ was used. However, as temperature increased, it was programmed to be adjusted by the control software to maintain a torque range of 0.5 to $5 \mathrm{~g} \cdot \mathrm{cm}$, allowing a maximum strain of $10 \%$ (still within the linear viscoelastic region) at high temperatures. Data points were collected every 5 seconds. Dynamic shear storage modulus $\left(G^{\prime \prime}\right)$, dynamic shear loss modulus $\left(G^{\prime \prime}\right)$, and their ratio $\tan \delta\left(=G^{\prime \prime} / G^{\prime}\right)$ were recorded using the Orchestrator ${ }^{\mathrm{TM}}$ software (TA Instruments Inc.).

\subsection{Shape Memory Behavior}

To quantify the shape memory behavior, constrained stress recovery tests were performed in compressive mode using the parallel plate fixture in ARES-LS2 rheometer (TA Instruments Inc.). One cylindrical sample of reticulated foam of approximately $\sim 20 \mathrm{~mm}$ diameter and $\sim 15 \mathrm{~mm}$ height was tested $\left(T_{g} \sim-87^{\circ} \mathrm{C}\right)$. The sample was first heated up to a temperature of $T_{g}+20^{\circ} \mathrm{C}\left(110^{\circ} \mathrm{C}\right)$ and deformed to an $80 \%$ compressive 
strain at a rate of $2.5 \mathrm{~mm} \cdot \mathrm{min}^{-1}$. Thereafter, the sample was cooled to $T_{g}-40{ }^{\circ} \mathrm{C}\left(40{ }^{\circ} \mathrm{C}\right)$ followed by heating back up to $T_{g}+20{ }^{\circ} \mathrm{C}$ while maintaining the $80 \%$ strain. At the end of the cool-heat cycle, the strain was released at a rate of $2.5 \mathrm{~mm} \cdot \mathrm{min}^{-1}$ and the recovered strain was obtained from the distance between the plates when the axial force dropped to $5 \mathrm{~g}$. The sample stress was monitored throughout the compress-cool-heat-release cycle, and three consecutive cycles were performed on the sample.

\section{B. Results and Discussion}

\section{Proposed Mechanism of Process Modifications}

The modifications in the foam synthesis process led to a significant reduction in the bulk density of the foam while maintaining an excellent structural uniformity. Specifically, we attempted to control the i) blowing agent amount $(7 \mathrm{ml}$, at 2.5 times of the previous use levels), ii) mold size (2.3 liters instead of 1 liter used previously), and iii) type of surfactant (2.8 g DC5943 (Air products Inc.) enabling better compatibility with the blowing agent and foaming solution relative to previously used DCI990 and DC5179), for achieving the objective of lower densities. Also a novel process change, involving slow introduction of vacuum during foaming, was incorporated to assist in the rise and stabilization of the foams. The estimated mechanism of action of these modifications is briefly discussed below: 


\subsection{Surfactants}

DC5943 is a water soluble silicone surfactant which is designed for high efficiency and cell uniformity in low density water blown polyether type foams. Both previously used surfactants DC 1990 and DC5179, in contrast, are water insoluble; DC1990 is a silicone surfactant for polyester based polyurethane formulations, and DC5179 is a mild surface stabilizing agent which is typically used in combination with a strong bulk stabilizer (Air Products Corp., Product data sheets). Hence, use of DC5943, was expected to facilitate a better chemical compatibility with the polymer solution based on a qualitative consideration of the cohesive energies of the monomers, surfactant and blowing agents. For determining the adequate level of the DC5943 surfactant, initially it was used at $2.8 \mathrm{~g}$ level (based on product data sheets, Air Products Inc.). Thereafter it was empirically optimized by fabricating foams with amounts of $\pm 2 \mathrm{~g}$, in steps of $0.4 \mathrm{~g}$, and comparing for foam quality (density and cell structure uniformity).

\subsection{Blowing Agent}

For achieving lower foam densities, it is essential to have generation of larger amount of gas during the foam rise, as well as its uniform entrapment throughout the polymer matrix [129]. While incorporation of $~ 2.5$ times more blowing agent in this modified process was critical to achieving the low densities, its uniform and stable dispersion through the foaming solution was possible, at least partly, due to the changes in the surfactant type. Surfactant optimization significantly improved the chemical stability of the foaming solution relative to previous formulations. Addition of such large 
amounts of blowing agent would have otherwise led to phase separation and generation of large voids in the foam bulk.

\subsection{Mold Size}

Mold size has previously been shown to have an effect on the final foam density [132]. In the view of the large volume expansion in the rising foam within relatively short time intervals (2-5 minutes), availability of more lateral space for expansion is expected to allow better foam rise. It is also likely to limit the effect of gravity by reducing the ratio of longitudinal to lateral dimension.

\subsection{Process Change}

Pulling vacuum on the rising foam is another technique to counter the effect of gravity and assist in the foam rise. Also, it was favorable to help cure the foam and strengthen the scaffold in the desired expanded state, thereby limiting the shrinkage of foams when they return to the atmospheric pressure environment.

\subsection{Other}

Other modifications such as, more efficient mixing via use of a speed-mixer (DAC 150 FVZ-K, FlackTek Inc.), and control over the foaming solution viscosity, were also employed in fabrication of these foams. Figure $\mathbf{3 3}$ gives a summary of all the process modifications and their point of action during the foam synthesis. Figure 34 and 
Figure 35 demonstrate the improvement in the foam density, dimensional stability, and structural uniformity as a result of these changes.

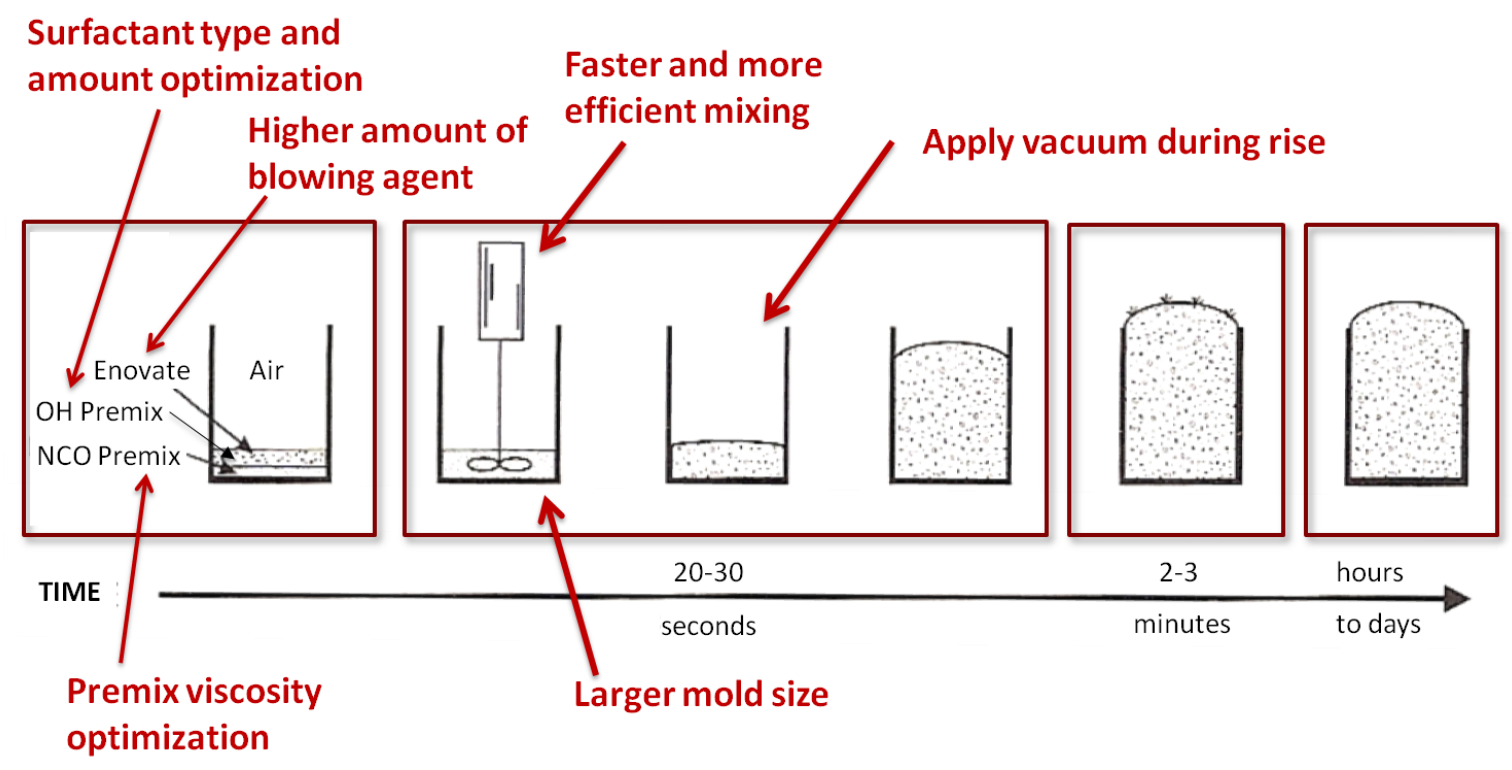

Figure 33: Summary of the process modifications employed in generation of lower density foams.
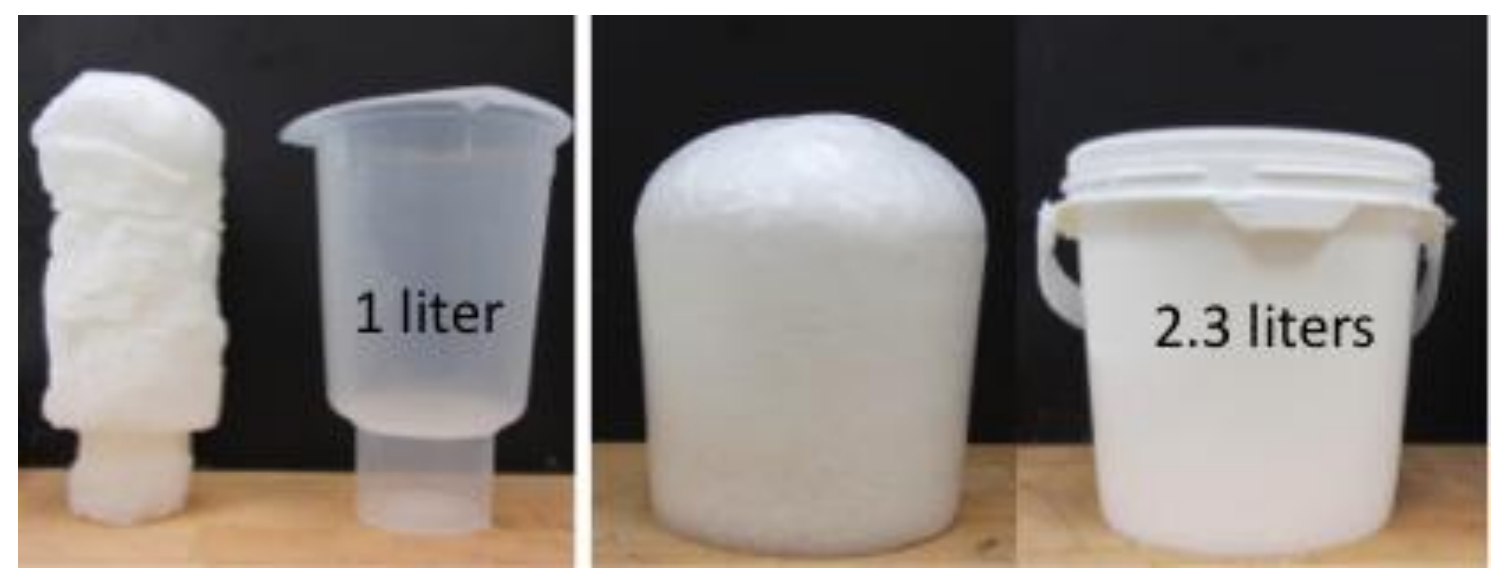

Figure 34: Comparison of the volume and dimensional stability of foams from the original process (left), to those from the modified process (right). 


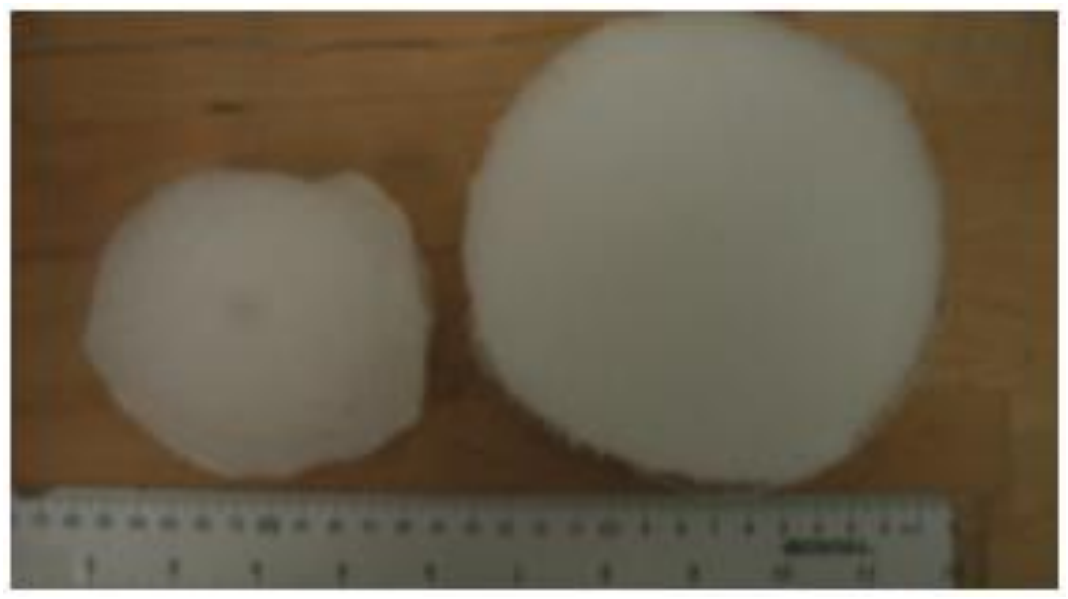

Figure 35: Cross section of foam from the original process (left), and the modified process (right). A visible improvement in the foam uniformity is observed.

\section{Proposed Mechanism of Post- processing by Plasma Treatment}

Plasma treatment of foams is not widely recognized for reticulation purposes. To our knowledge, one instance in which plasma has been referenced for this end use is a US patent application by Hild et al. [184]. Here polyimide based foams, aimed at achieving a range of insulation capabilities, were suggested to be plasma treated for reticulation. In another instance, Mitsubishi SMP foams were treated with plasma for sterilization purposes, and removal of some cell membranes was observed during the procedure [23, 38]. However, this process was not pursued specifically for foam reticulation.

Plasma is a fundamental state of matter that lacks a definite shape, and is comprised of an electrically neutral medium of charged particles, that are electrically conductive, produce magnetic fields and electric currents, and respond strongly to electromagnetic forces [185]. When a polymer surface is exposed to plasma, the polymer 
chains are bombarded by the constituent energetic radicals. This is followed by a chemical reaction of the polymer chains that come in contact with these reactive species, and can be manipulated towards multiple end results of exposing or grafting several function groups on the polymer surface.

$\mathrm{O}_{2} / \mathrm{CF}_{4}$ Plasma is a common etch chemistry, which is thought to produce the oxyfluoro radical that functions as an aggressive etchant. On exposure to these radicals, polymer chains get oxidized and begin to break down into smaller fragments- a phenomenon that progresses further on continued exposure. Ultimately, these fragments become small enough that they can get volatized, leading removal of the material. In other words, it is effectively a controlled oxidation (or controlled burning) process. As the residual cell membranes have a high surface area and much lower thickness compared to cell struts, they get volatized first, leaving a reticulated cell structure behind. Further, the ultra low density of these foams limits the dampening of the plasma energy, and permits good reticulation depths via this process.

\section{Foam Characterization}

\subsection{Density and Cell Structure}

A density of $0.0083 \mathrm{~g} \cdot \mathrm{cm}^{-3}$ and $0.0076 \mathrm{~g} \cdot \mathrm{cm}^{-3}$ was achieved for as-made and reticulated foams respectively (Table 12). This signifies a huge improvement compared to previously reported foams, and portends significantly enhanced embolic efficiency. A uniform cell structure was observed, as seen in Figure 36 and Figure 37. Further, 
complete removal of residual membranes is witnessed in the foams after plasma treatment (Figure 36 and Figure 37).
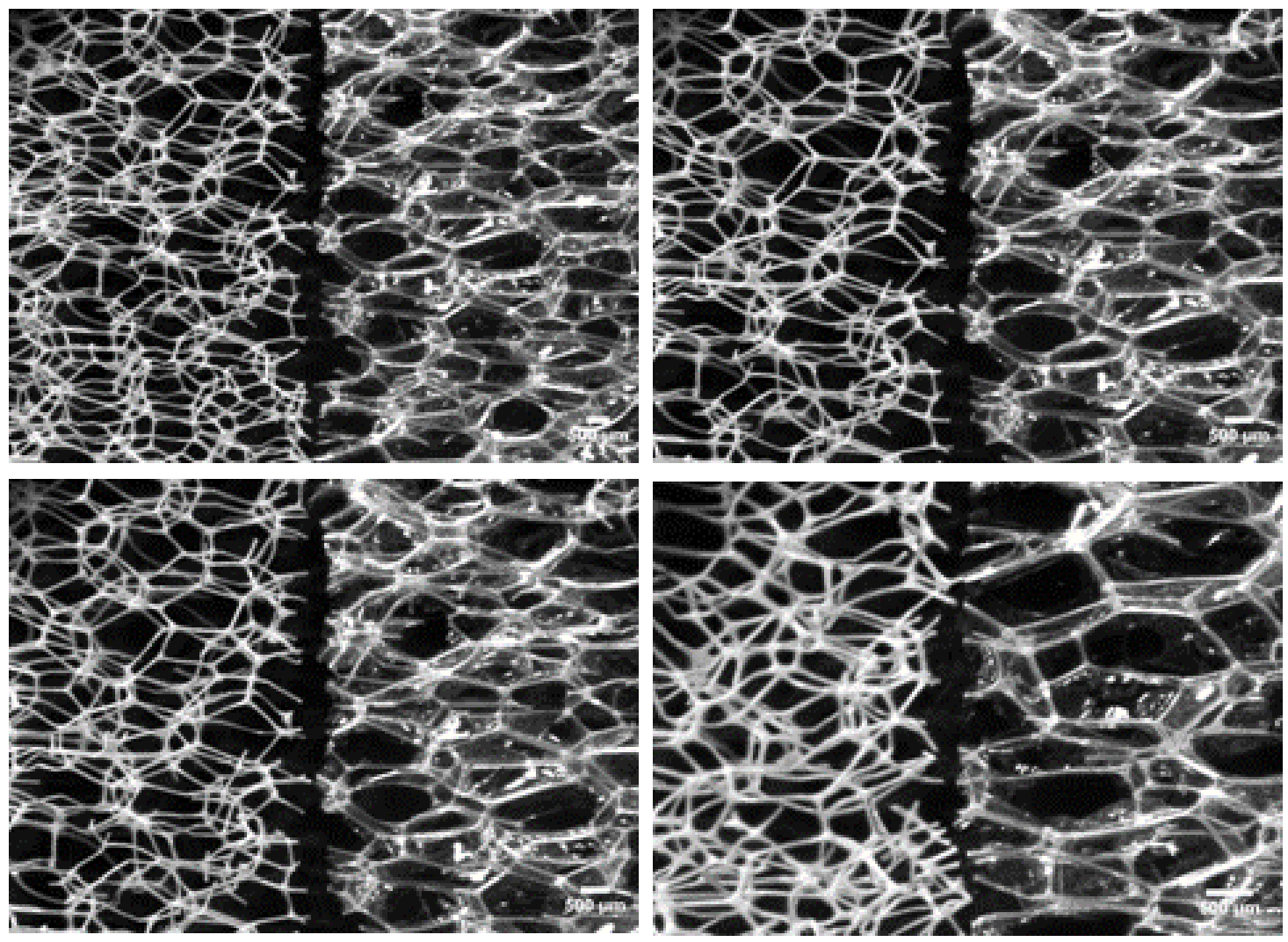

Figure 36: Foam cell morphology for as-made (right) and reticulated (left) samples, as captured through an optical microscope. Scale bar $=500 \mu$. 

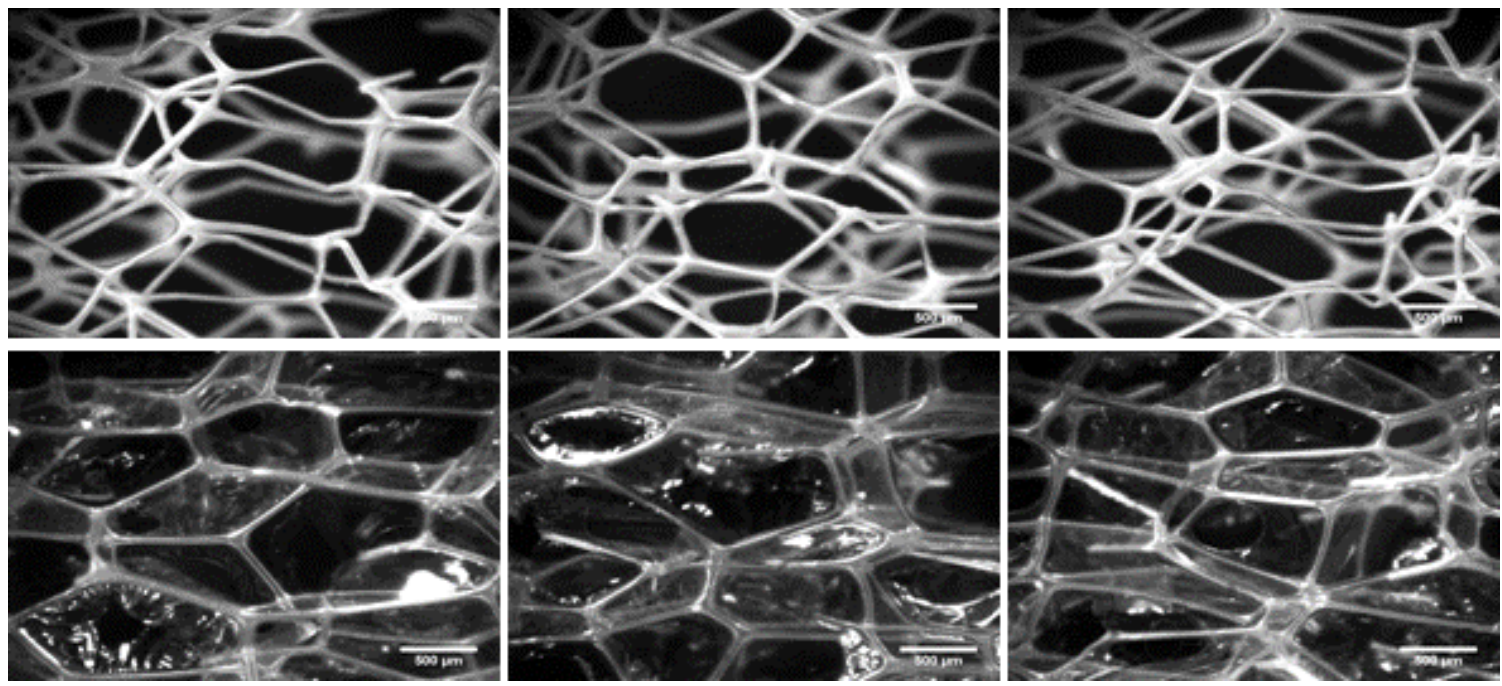

Figure 37: Foam cell morphology for reticulated (top) and as-made (bottom) samples, as captured through an optical microscope. Scale bar $=500 \mu$.

Table 12: Summary of foam characterization results before and after plasma treatment.

\begin{tabular}{|c|c|c|}
\hline Property & As-made (non-reticulated) & Reticulated \\
\hline Density $\left(\mathbf{g} \cdot \mathbf{c m}^{-3}\right)$ & 0.0083 & 0.0076 \\
\hline $\mathbf{G}^{\prime}$ glassy $(\mathbf{k P a})$ & $62.23 \pm 7.59$ & $31.46 \pm 5.84$ \\
\hline $\mathbf{G}^{\prime}$ rubbery $(\mathbf{k P a})$ & $2.03 \pm 0.33$ & $0.89 \pm 0.31$ \\
\hline $\boldsymbol{T}_{\boldsymbol{g}}\left({ }^{\circ} \mathbf{C}\right)$ & $83.7 \pm 1.1$ & $87.0 \pm 0.5$ \\
\hline $\boldsymbol{T a n} \boldsymbol{\delta}($ peak value $)$ & $0.94 \pm 0.04$ & $0.95 \pm 0.14$ \\
\hline
\end{tabular}

\subsection{DMTA}

Dynamic mechanical analysis results of the foam samples are shown in Table 12 and Figure 38. A reduction in the glassy and rubbery moduli of the foams by a factor of $\sim 2$ is observed. This is expected from the removal of material during the plasma 
treatment, and the reduced foam density. A small increase of $\sim 3{ }^{\circ} \mathrm{C}$ was also recorded in the $T_{g}$ after plasma treatment. However, the features of DMA curve characteristic of shape memory behavior, such as sharp transition and large modulus drop across the transition were largely intact.

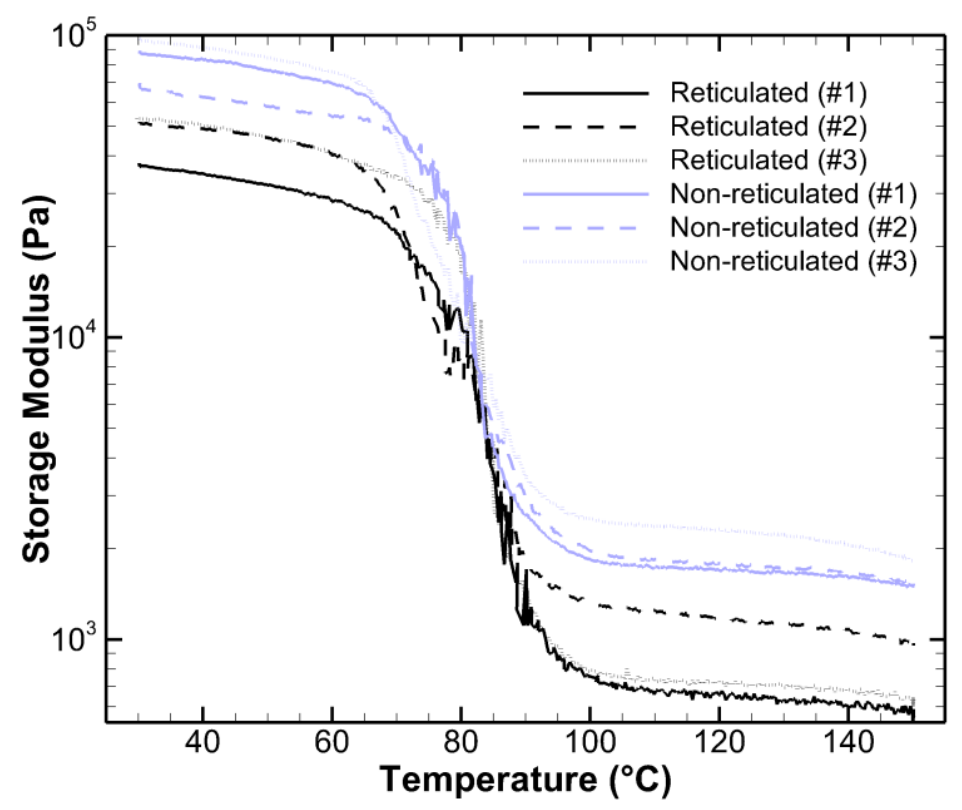

Figure 38: Dynamic mechanical analysis of foams for as-made and reticulated samples.

\subsection{Shape Memory Behavior}

Shape memory behavior of the foams is shown in Figure 39. Excellent shape recovery of $\sim 99 \%$ was observed for all the three cycles for reticulated foams, with negligible hysteresis across cycles. This is in sharp contrast to the shape memory behavior seen with related foams in the previous work [30], where the first 2-3 cycles invariable show a relatively high hysteresis. It is estimated that the energy consumed in 
rupturing the cell membranes, during the initial compression stage, forms a major component of the hysteresis in these foams. Hence the lack of membranes in the reticulated foams is likely responsible for a significantly improved shape memory behavior.
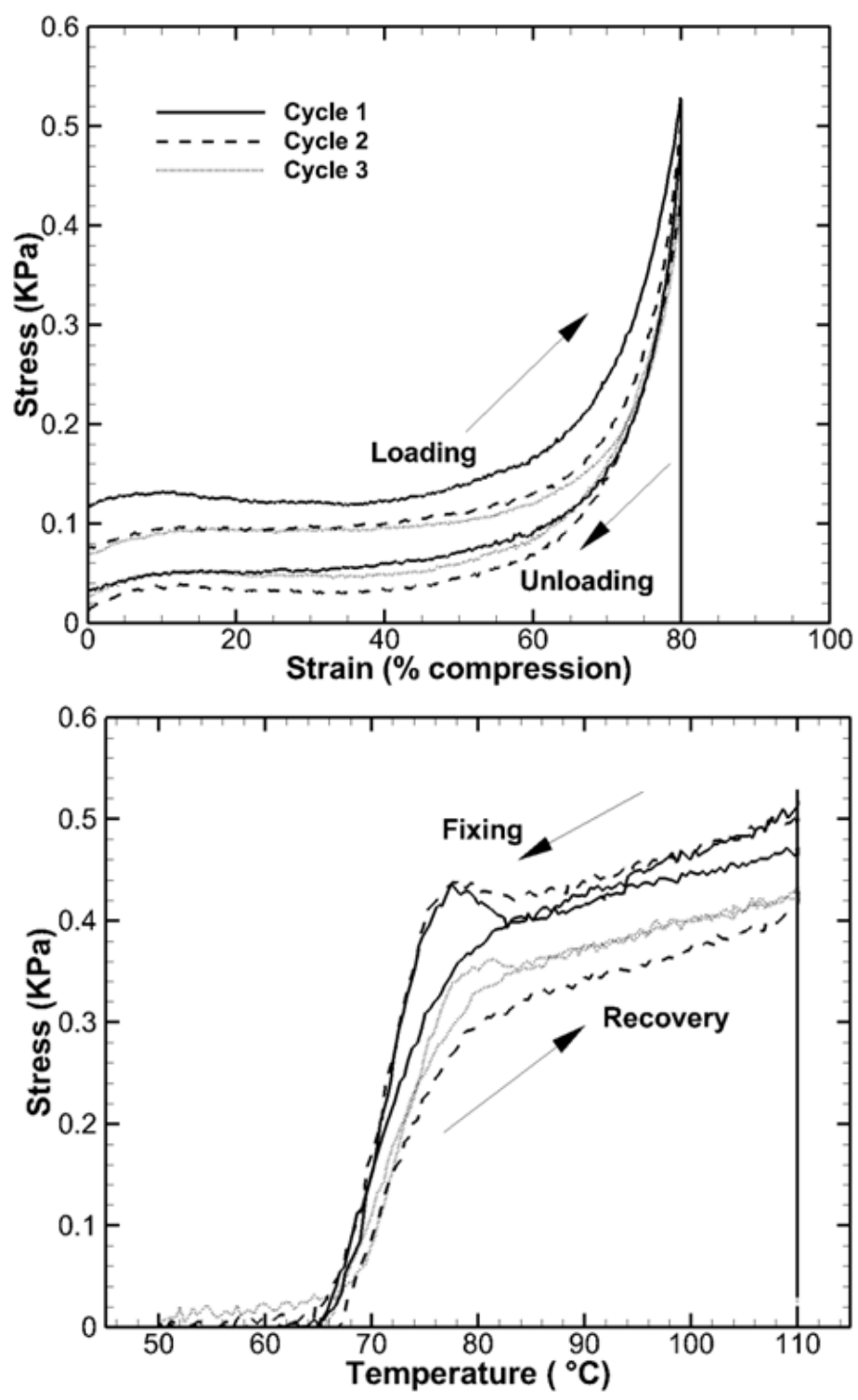

Figure 39: Shape memory behavior of the reticulated foams. 


\section{Conclusions}

There is a persistent need of sufficiently low density SMP foams with a uniform cell structure and good mechanical properties, for development of high efficiency, minimally invasive embolic biomedical devices. Further, it is required to precisely control the foam morphology, because the as-made foams typically have a combination of open, closed, and partially open cells in their structure which pose an imminent danger in blood contacting biomedical applications. Namely, they can get dislodged causing an emboli downstream, and/or restrict better permeation of blood/physiological fluid through the foam device post-implantation.

This chapter focused on further improvement of the ultra low density SMP foams, discussed in previous chapters, with respect to two specific aspects: a) fabrication of SMP foams with an exceptionally low density, $\left(\sim 0.008 \mathrm{~g} \cdot \mathrm{cm}^{-3}\right)$ via a multitude of additives and process advancements, and b) achieving complete reticulation of these materials using a state-of-the-art plasma treatment process on as-made foams. Further it was confirmed that the reticulated foams showed improved shape memory behavior compared to the as-made foams, with reduced hysteresis across the thermo-mechanical cycles. The advancements reported here herald a new generation of "smart" biomedical scaffolds for regenerative or embolic applications requiring high filling efficiency and controlled permeability during implantation. 


\section{CHAPTER V}

\section{BIODEGRADABLE FOAMS}

Previous chapters discussed the development of a new class of "smart" SMP foams with highly covalently crosslinked polymer structure for maintaining excellent mechanical and shape memory properties at the application-specific ultra low densities, as well as achieving extremely low densities and controlled reticulation of the foam cell surfaces. Several studies critical to the application of these materials in embolic treatment of aneurysms, such as of their mechanical properties and shape memory behavior [30], inclusion of contrast agent for enabling in vivo imaging during device delivery [54], stresses experienced on the aneurysm wall during actuation/implantation [63], effect of moisture on the foam's actuation temperature, [55] variation in composition to control the rate of actuation of foams in presence of moisture [29], and in vivo implantation in porcine models [53] have been performed in the recent years. Based on the promising outlook of these materials as reported in these studies, it was further desirable to make them biodegradable. It was hypothesized that if the implant degraded over time after the initial endothelialization of the aneurysm neck, the isolated aneurismal bulge would eventually atrophy, leading to a complete cure of the vasculature. Hence this chapter is aimed at further expanding the utility of these biomaterials, as implantable low density SMP foams, by introducing controlled biodegradability. 
In order to achieve biodegradability, we developed a series of low density foams using polycaprolactone triol (PCL-t, $\mathrm{M}_{\mathrm{n}} 900 \mathrm{~g}$ ). PCL-t was a monomer of choice for introducing hydrolytically degradable ester linkages in these materials, both because it is a trifunctional monomer, in agreement with the desired highly covalently crosslinked network structure [30], and because polycaprolactone (PCL) is an FDA approved polymer that is extensively used in tissue engineering applications such as the regeneration of bone [186], ligament [187], skin [188, 189], nerve [190, 191], and vascular tissues [192]. Although a relatively low rate of degradation (2-3 years) has been observed in PCL based materials due to its high crystallinity, this degradation rate is tunable and has been shown to increase with decreasing crystallinity [193].

Here we report the synthesis process of these covalently crosslinked and amorphous degradable SMP foams based on PCL-t, HPED, TEA, and water as the hydroxyl monomers. Control over the rate of degradation was attempted by a) changing the concentration of the degradable PCL-t monomer, and b) introducing isocyanates of higher hydrophobicity, TMHDI and Isophorone diisocyanate (IPDI), in addition to HDI. Increase in the hydrophobicity of the monomers was of interest not only for its effect on the rate of degradation, but also for limiting the rate of plasticization of the polymer in physiological media, in order to enable transcatheter delivery of the device, as discussed in Chapter III [29]. Characterization of density, cell structure, thermochemical properties, shape memory, and degradation rate are reported and discussed with respect to an ideal device for aneurysm treatment. 


\section{A. Materials and Methods}

\section{Synthesis of Foams}

Foam compositions are given in Table 13. All chemicals, PCL-t $\left(M_{n} 900 \mathrm{~g}\right.$, Sigma Aldrich Inc.), HPED (99\%, Sigma Aldrich Inc.), TEA (98\%, Alfa Aesar Inc.), HDI (TCI America Inc.), TMHDI (TCI America Inc.), IPDI (TCI America Inc.), and DI water ( $>17 \mathrm{M} \Omega \cdot \mathrm{cm}$ purity, Millipore) were used as received (Figure 40). Foams were synthesized in a three-step method as previously reported [30]. Inclusion of the ester bonds in polymer composition however required significant optimization of the type and amount of surfactants and catalysts for viable foams, relative to previously reported formulations. Optimized levels of amine based catalyst (BL-22, Air Products Corp.), tin based catalyst (T-131, Air Products Corp.) and silicone based surfactants (DCI990 and DC5179, Air Products Corp.) as used for foam synthesis, are reported in Table 13. Foam compositions 100P-HDI, 50P-HDI, 25P-HDI and OP-HDI refer to formulations with $100 \%, 50 \%, 25 \%$ and $0 \%$ (control) of hydroxyl equivalents from PCL-t, and the remaining from HPED and TEA in a 2:1 ratio. This denomination excludes the $41 \%$ of isocyanate equivalents that are consumed in reaction with water (chemical blowing agent), while maintaining a 104 isocyanate index in the net foam formulation. The foam

compositions 100P-TMHDI and 100P-IPDI refer to formulations where HDI is completely replaced with TMHDI and IPDI respectively in the 100P-HDI foams (Table 13). 
<smiles>CC(O)CN(CCN(CC(C)O)CC(C)O)CC(C)O</smiles>

HPED<smiles>[R]OCC(CC)(CO[R])CO[R]</smiles><smiles>OCCN(CCO)CCO</smiles>

TEA<smiles>[R]C(C)(C)C(=O)CCCCCOC(C)C</smiles>

Figure 40: Molecular structures of the polyfunctional hydroxyl monomers used in the synthesis of biodegradable foams.

Table 13: Composition of different foams fabricated in the study. 0P-HDI, 25PHDI, 50P-HDI and 100P-HDI denote an increasing concentration of the biodegradable monomer (PCL-t). 100P-TMHDI and 100P-IPDI denote substitution of HDI in the 100P-HDI composition with an equivalent amount of TMHDI and IPDI respectively.

\begin{tabular}{|c|c|c|c|c|c|c|c|c|c|c|c|}
\hline Sample ID & $\begin{array}{c}\text { HDI } \\
\text { (wt.\%) }\end{array}$ & $\begin{array}{l}\text { TMHDI } \\
\text { (wt.\%) }\end{array}$ & $\begin{array}{c}\text { IPDI } \\
(\text { wt. \%) }\end{array}$ & $\begin{array}{l}\text { HPED } \\
\text { (wt.\%) }\end{array}$ & \begin{tabular}{|c|} 
TEA \\
$(w t . \%)$
\end{tabular} & $\begin{array}{l}\text { PCL-t } \\
\text { (wt.\%) }\end{array}$ & $\begin{array}{l}\text { Water } \\
\text { (wt.\%) }\end{array}$ & $\begin{array}{l}\text { DC5179 } \\
\text { (wt.\%) }\end{array}$ & $\begin{array}{c}\text { DCI990 } \\
\text { (wt.\%) }\end{array}$ & $\begin{array}{l}\text { T-131 } \\
\text { (wt.\%) }\end{array}$ & $\begin{array}{l}\text { BL-22 } \\
\text { (wt.\%) }\end{array}$ \\
\hline $\begin{array}{l}\text { 0P-HDI } \\
\text { (Control) }\end{array}$ & 62.85 & - & - & 19.68 & 6.68 & 0.00 & 2.76 & 3.82 & 3.31 & 0.26 & 0.64 \\
\hline 25P-HDI & 50.29 & - & - & 12.03 & 4.08 & 24.67 & 2.21 & 2.75 & 3.00 & 0.28 & 0.70 \\
\hline 50P-HDI & 42.19 & - & - & 6.73 & 2.28 & 41.39 & 1.85 & 1.91 & 2.55 & 0.31 & 0.79 \\
\hline 100P-HDI & 32.07 & - & - & - & - & 62.93 & 1.41 & - & 2.23 & 0.39 & 0.98 \\
\hline 100P-TMHDI & - & 37.12 & - & - & - & 58.25 & 1.30 & - & 2.06 & 0.36 & 0.90 \\
\hline 100P-IPDI & - & - & $\begin{array}{c}38.4 \\
2\end{array}$ & - & - & 57.04 & 1.28 & - & 2.02 & 0.35 & 0.89 \\
\hline
\end{tabular}

The wt.\% value represents the net amount of the component in the final foamed polymer. 


\section{Characterization of Physical Properties}

\subsection{Density and Cell Structure}

Core density of the foam was measured from the representative top and middle sections of the foam as per the ASTM standard D-3574-08. Average values over 5 samples are reported for each composition. Cell structure was characterized using a low vacuum scanning electron microscope (NeoScope JCM 5000, Nikon). Foam samples were mounted on the stage and their surface morphology was captured at $5 \mathrm{kV}$ accelerating voltage and 50X magnification.

\subsection{DSC}

$T_{g}$ was measured using a Pyris Diamond DSC (PerkinElmer Inc.). A 3-5 mg sample was loaded in a vented aluminum pan at room temperature, cooled to $-60{ }^{\circ} \mathrm{C}$ and then run through a heat - cool - heat cycle from $-60{ }^{\circ} \mathrm{C}$ to $120^{\circ} \mathrm{C}$ at a rate of $20^{\circ} \mathrm{C} \cdot \mathrm{min}^{-1}$. The half-height of the transition during the second heat was taken as an estimate of the $T_{g}$

\subsection{FTIR}

An FTIR spectrum of the foams was collected using a Spectrum 2000 FTIR (Perkin Elmer Inc.). The sample chamber was purged with nitrogen gas for 5 minutes and a background spectrum was captured. Thereafter, thin slices ( 2-4 $\mathrm{mm}$ in thickness)

cut from foam blocks were placed in a $2 \mathrm{~cm}^{-1}$ JStop holder $(0.88 \mathrm{~cm}$ aperture $)$. The 
chamber was again purged with nitrogen for 5 minutes after placing the sample, and an FTIR spectrum was collected in the absorption mode at a resolution of $4 \mathrm{~cm}^{-1}$. The test was performed in duplicate to ascertain reproducibility of the spectra. A total of 50 scans were taken for each sample and the background spectrum was subtracted using the Spectrum software (Perkin Elmer Inc.). Also, a baseline correction was performed on the resulting spectra to account for the scattering of the IR beam across the sample.

\subsection{Shape Memory Behavior}

To quantify the shape memory behavior, constrained stress recovery tests were performed in compressive mode using the parallel plate fixture in ARES-LS2 rheometer (TA Instruments Inc.). Five cylindrical 100P HDI foam samples of $\sim 20 \mathrm{~mm}$ diameter and $\sim 15 \mathrm{~mm}$ height were tested $\left(T_{g} \sim-20^{\circ} \mathrm{C}\right)$. The sample was first heated up to a temperature of $T_{g}+40{ }^{\circ} \mathrm{C}\left(20{ }^{\circ} \mathrm{C}\right)$ and deformed to a $60 \%$ compressive strain at a rate of $2.5 \mathrm{~mm} \cdot \mathrm{min}^{-1}$. Thereafter, the sample was cooled to $T_{g}-30{ }^{\circ} \mathrm{C}\left(-50{ }^{\circ} \mathrm{C}\right)$ followed by heating back up to $T_{g}+40{ }^{\circ} \mathrm{C}$ while maintaining the $60 \%$ strain. At the end of the cool-heat cycle, the strain was released at a rate of $2.5 \mathrm{~mm} \cdot \mathrm{min}^{-1}$ and the recovered strain was obtained from the distance between the plates when the axial force dropped to $5 \mathrm{~g}$. The sample stress was monitored throughout the compress-cool-heat-release cycle, and five consecutive cycles were performed on each of the five tested samples. Also, the final recovered sample height post cyclic testing was measured after a relaxation period of 24 hours at $T_{g}+40^{\circ} \mathrm{C}\left(20^{\circ} \mathrm{C}\right)$ to estimate the time dependence of the shape recovery. 


\section{Characterization of Degradation Behavior}

For testing the degradation behavior of the materials, degradation in both alkaline medium (0.1 N NaOH, accelerated degradation) and phosphate buffer saline medium (PBS, physiological degradation) were performed. Rectangular $(1 \mathrm{~cm}$ by $2 \mathrm{~cm})$ strips of foam 2-4 $\mathrm{mm}$ in thickness were cut from the representative areas of the foam stock. The foam samples were cleaned using a previously reported protocol for removal of residual foaming additives and partial rupture of cell membranes [29]. Briefly, the samples were soaked in $0.1 \mathrm{~N} \mathrm{HCl}$ solution under sonication for 2 hours, and then cleaned for two 15 minute cycles each with a) 80-20 volume\% solution of DI water and Conrad 70, and b) DI water, under sonication. Thereafter, the cleaned foams were dried under vacuum at $50{ }^{\circ} \mathrm{C}$ overnight.

The degradation study was conducted for a period of 7 months with analysis of mass loss and changes in the physical and chemical structures of the materials conducted weekly for the first 3 months, followed by once every $\sim 3$ weeks. First, the initial weight, FTIR, and gross morphology of the samples were recorded. Thereafter, they were placed in a $\sim 1000$ times weight excess of the media in labeled vials at $37{ }^{\circ} \mathrm{C}$. Vacuum was pulled on the submerged samples overnight ( 12 hours) for removal of air bubbles that may otherwise inhibit the contact of media with the polymer. Following this period the vacuum was released, and the vials were refilled to account for any loss in media due to vacuum. It was ensured that all the samples were completely submerged in water and were void of any air bubbles. Samples remained in the oven at $37{ }^{\circ} \mathrm{C}$ for the next 6 days to undergo degradation. After this degradation period, the media was removed and the 
samples were rinsed in DI water, followed by drying under vacuum at $50{ }^{\circ} \mathrm{C}$ overnight. Characterization of the dried samples was performed prior to repeating their submersion in the degradation media.

\subsection{Mass Loss}

For estimating the pattern of mass loss of the polymers, ten samples of each composition and each media type were tested. An Ohaus Analytical Plus scale (AP250D, Central Carolina Scale Inc.) with a resolution of $0.01 \mathrm{mg}$ was used for the weight measurements. Mass of samples was recorded initially $\left(W_{i}\right)$, and after every week $(n)$ of degradation $\left(W_{n}\right)$. The $\%$ degradation at the $n^{\text {th }}$ week was calculated as:

$$
\% \text { mass loss }=\left(\frac{W_{i}-W_{n}}{W_{i}}\right) \times 100 ; n=1 \text { to } 28
$$

The average $\%$ mass loss and standard deviations calculated over the 10 samples were reported.

\subsection{FTIR}

Foam FTIR spectra were collected using a Spectrum 2000 FTIR (Perkin Elmer Inc.) according to the procedure detailed above (Section 2.4). Spectral changes were monitored as a function of degradation time with particular attention given to ester, urethane, and urea absorbance bands. The spectra were normalized with respect to the alkyl absorbance at $2857 \mathrm{~cm}^{-1}$ for analysis. However, as the alkyl groups may not remain conserved throughout the degradation period for all compositions, only a qualitative 
analysis of the shift in peak absorbance was performed to estimate the mechanism of degradation.

\subsection{Gross Morphology}

Variation in the gross morphology of the foams was captured using a camera (SX230HS, Canon Inc.). An analysis of visible morphological changes due to the sample degradation was performed from the images.

\section{B. Results and Discussion}

\section{Physical Properties Characterization}

\subsection{Density and Cell Structure}

Density values of different foam compositions are reported in Table 14. Foam densities ranging from 0.020 to $0.093 \mathrm{~g} \cdot \mathrm{cm}^{-3}$ were recorded. These values correspond to an average porosity of greater than $90 \%$ for all compositions, $\left[\right.$ porosity $\left.=\left(\rho_{\text {neat }}-\rho_{\text {porows }}\right) / \rho_{\text {neat }}\right]$; here $\rho_{\text {porows }}$ is the foam density and average $\rho_{\text {neat }} \sim 1.1 \mathrm{~g}^{*} \mathrm{~cm}^{-3}$ is the neat/unfoamed polymer density. The relatively small standard deviation $\left(0.002-0.015 \mathrm{~g} \cdot \mathrm{cm}^{-3}\right)$ in densities indicates good uniformity of the individual foam samples. 
Table 14: Average foam densities and glass transition temperatures for the different biodegradable and control compositions.

\begin{tabular}{|c|c|c|}
\hline Sample ID & Density $\left(\mathbf{g}^{\cdot \mathbf{c m}^{-3}}\right)$ & $\boldsymbol{T}_{\boldsymbol{g}}\left({ }^{\mathbf{0}} \mathbf{C}\right)$ \\
\hline 0P-HDI (control) & $0.020 \pm 0.002$ & 70 \\
\hline 25P-HDI & $0.025 \pm 0.005$ & 29 \\
\hline 50P-HDI & $0.047 \pm 0.015$ & -19 \\
\hline 100P-HDI & $0.036 \pm 0.002$ & -11 \\
\hline 100P-TMHDI & $0.093 \pm 0.012$ & 26 \\
\hline 100P-IPDI & $0.035 \pm 0.005$ & \\
\hline
\end{tabular}

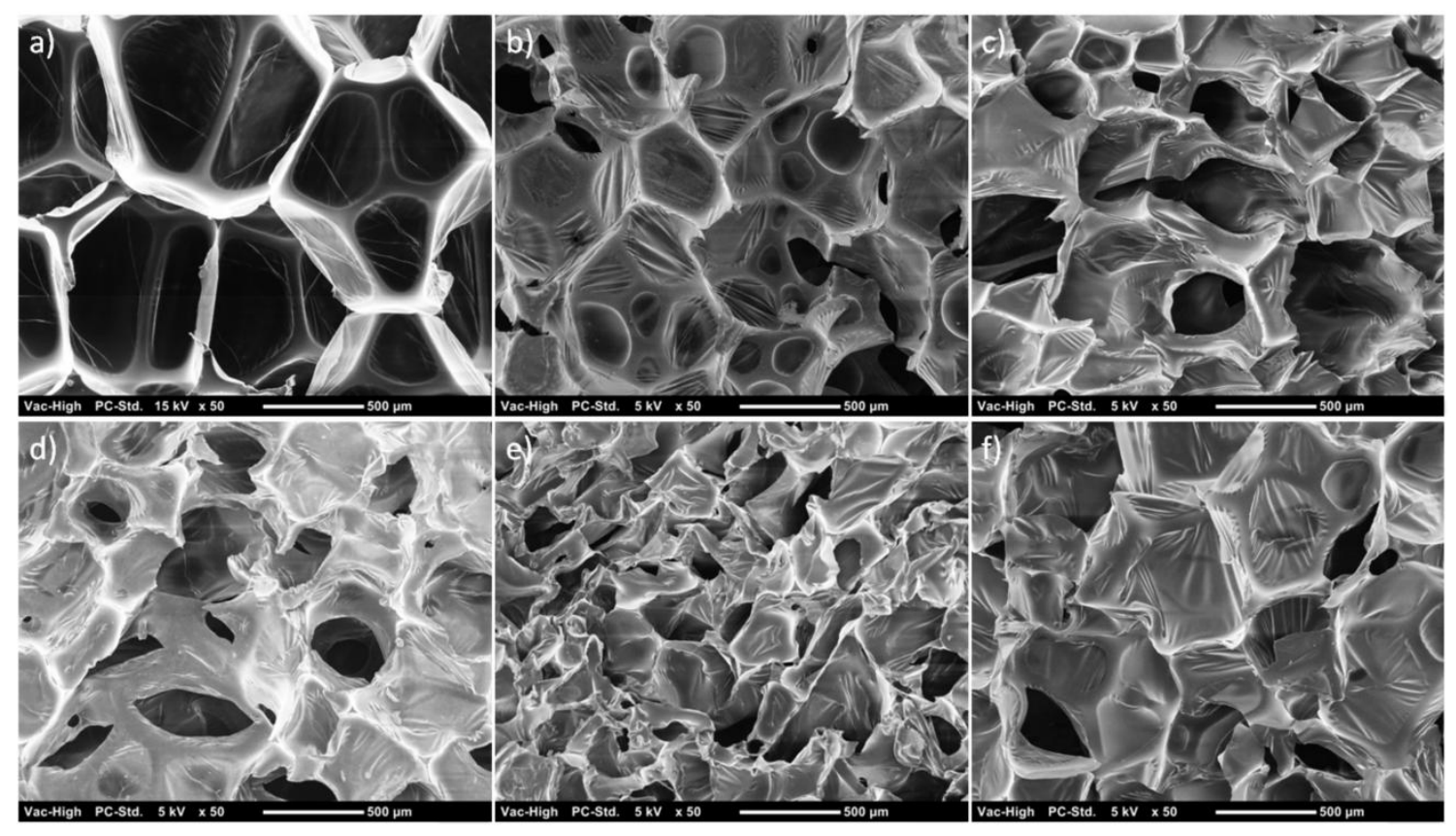

Figure 41: Cell structure as seen from Scanning Electron Microscopy for a) 0PHDI, b) 25P-HDI, c) 50P-HDI, d) 100P-HDI, e) 100P-TMHDI, and f) 100P-IPDI foam compositions. A mixed open to closed cell morphology, with uniform cell structure is observed for all compositions. Scale bar $=500 \mu$. 
Cell structure of the foams is shown in Figure 41. A closed, or mixed closed to open, cell structure is observed for these foams. Thin residual membranes, similar to those reported for related materials previously [29, 30], are seen on the cell surfaces. Overall, the images depict a high level of uniformity in the cell structure of a given foam composition. This is largely attributed to the use of gas blowing process for foam fabrication, as well as optimization of the foaming additives and process parameters. For instance, fabrication of related polyurethane foams from PCL-t and HDI using the High Internal Phase Emulsion (HIPE) templating process by David et al. produced comparatively higher densities $\left(0.21 \mathrm{~g} \cdot \mathrm{cm}^{-3}\right)$, with large variation in pore size ranging from $\sim 1 \mathrm{~mm}$ (from the $\mathrm{CO}_{2}$ bubbles during reaction of isocyanate with water) to $\sim 0.1-100 \mu[125]$.

\subsection{DSC}

$T_{g}$ values of different foam compositions are reported in Table 14 and Figure 42. The $T_{g}$ of foams was seen to decrease with increase in the PCL-t content from $70{ }^{\circ} \mathrm{C}$ (OP-HDI) to $-19{ }^{\circ} \mathrm{C}$ (100P-HDI). This is expected as the trifunctional PCL-t molecule can increase the free volume in the amorphous polymer network due to its longer branches relative to HPED and TEA. This increase in free volume can consequently increase the mobility of the polymer chains, and reduce the polymer $T_{g}$. Also replacing the HDI in the 100P-HDI composition with TMHDI and IPDI was seen to increase the $T_{g}$ of the foams. TMHDI is expected to increase the $T_{g}$ of the foams relative to HDI as the methyl groups of TMHDI can increase the energy required by the molecule to rotate 
about the backbone bonds. However, its effect is relatively small $\left(\sim 8^{\circ} \mathrm{C}\right)$. IPDI is seen to increase the foam $T_{g}$ relative to HDI by a much larger extent of $\sim 45{ }^{\circ} \mathrm{C}$. This may be attributed to the significant increase in the stiffness of the backbone due to the presence of the saturated ring of IPDI in the isocyanate molecule. It is noteworthy that a single glass transition is seen for all materials. Absence of any secondary transition indicates an amorphous network structure, and formation of an all-encompassing crosslinked network with no isolated pockets of unreacted monomer that were reported for related compositions by David et al. [125].

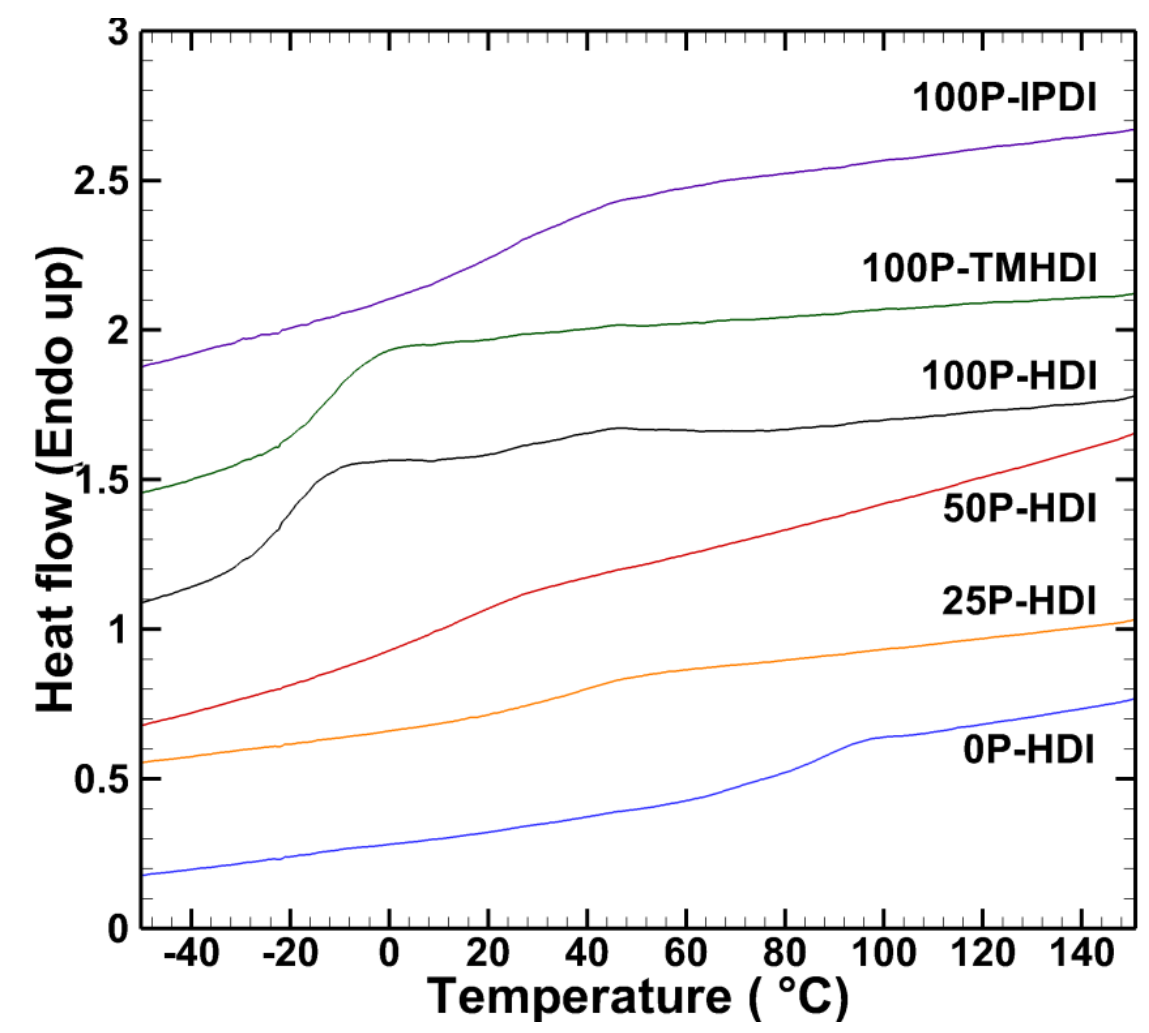

Figure 42: DSC curves of various foam compositions show a single $T_{g}$ indicating an amorphous polymer network structure. 


\subsection{FTIR}

FTIR spectra of foams are shown in Figure 43 and Figure 44, and labeled to highlight peaks corresponding to ester, urethane and alkyl groups that were utilized in the degradation analysis. The urethane carbonyl band was observed in the range of $1695-1700 \mathrm{~cm}^{-1}$ range for all compositions [162]. It is clearly visible in the OP-HDI through 50P-HDI compositions; however, it was masked by the ester peak in the 100PHDI, 100P-TMHDI and 100P-IPDI compositions. The urethane $\delta(\mathrm{N}-\mathrm{H})+v(\mathrm{C}-\mathrm{N})$ amide II and amide III bond absorbance were observed at $1530-1540 \mathrm{~cm}^{-1}$ and $1235-1250 \mathrm{~cm}^{-1}$, respectively. A urea shoulder from reaction of isocyanate with water (chemical blowing agent) was observed at $\sim 1630-1650 \mathrm{~cm}^{-1}$ for all compositions [162]. The ester carbonyl

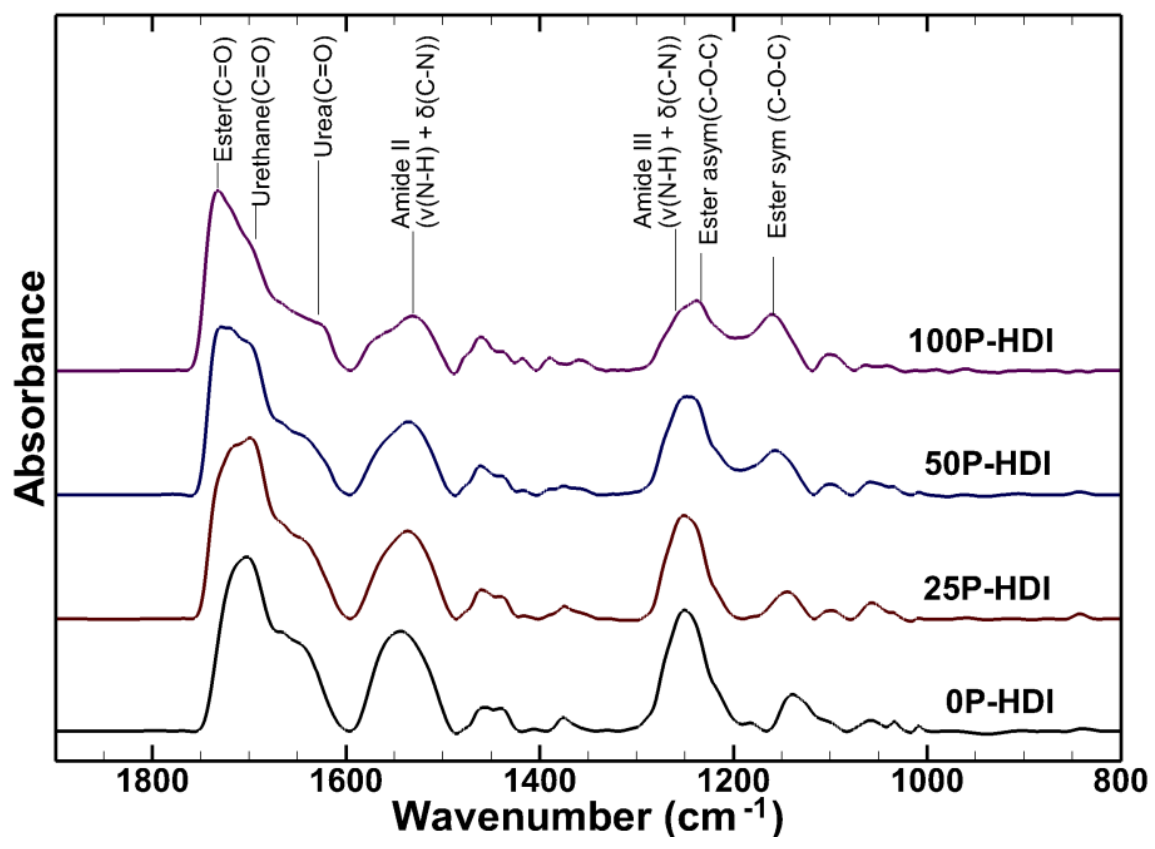

Figure 43: FTIR spectra of the samples with varying PCL-t content prior to degradation. Peaks corresponding to the ester, urethane and urea bonds are labeled for identification of respective trends. 


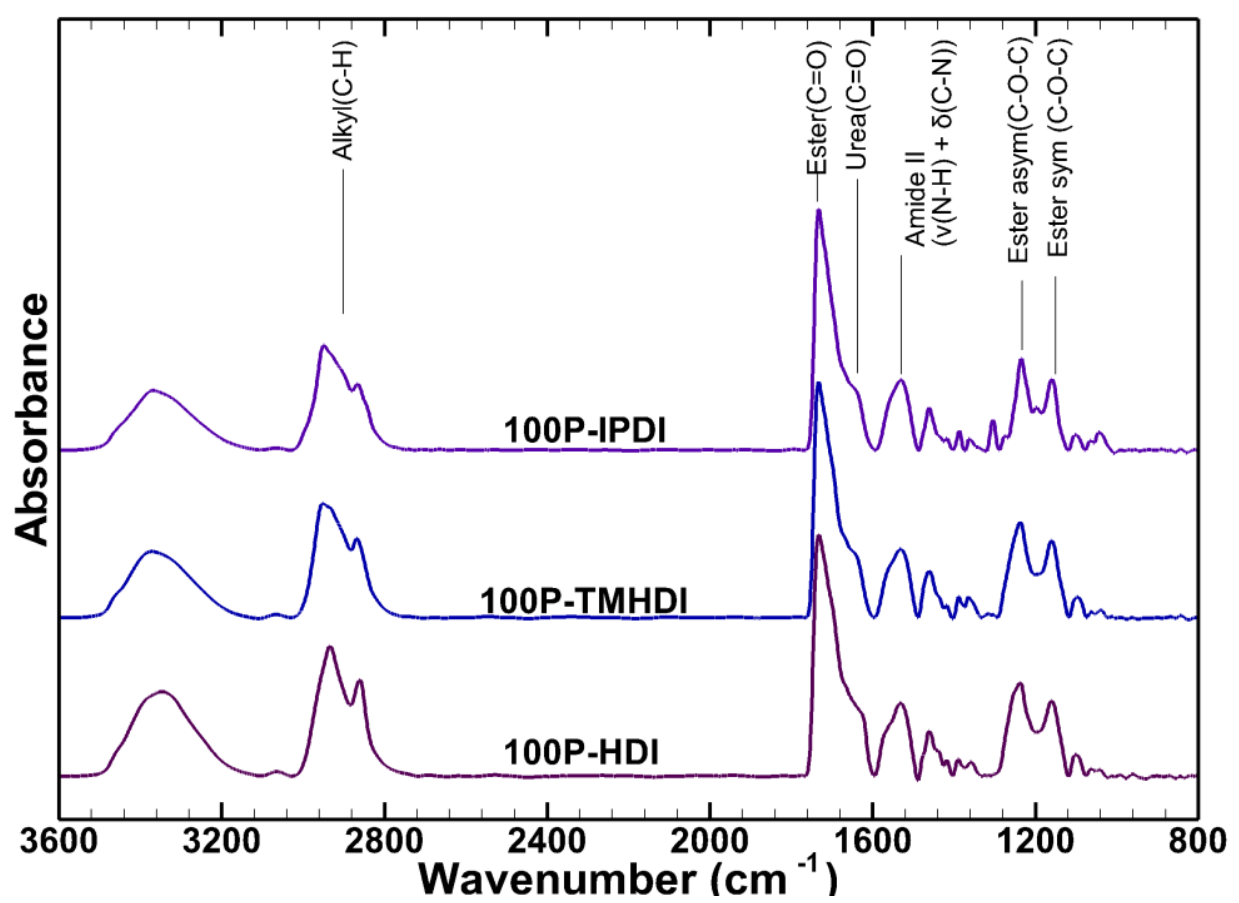

Figure 44: FTIR spectra of samples with the varying isocyanate type prior to degradation. Peaks corresponding to the alkyl, ester, urethane, and urea bonds are labeled for identification.

absorbance at $1730 \mathrm{~cm}^{-1}$ increased with increasing PCL-t content relative to the urethane carbonyl [162]. Similarly, the ester asymmetric $v(\mathrm{C}-\mathrm{O}-\mathrm{C})$ absorbance at $1236 \mathrm{~cm}^{-1}$ and the symmetric $v(\mathrm{C}-\mathrm{O}-\mathrm{C})$ ester absorbance is seen at $1160 \mathrm{~cm}^{-1}$ also increased with increasing PCL-t content [162]. The changes in the spectra from $\mathrm{CH}_{3}$ groups of TMHDI, and saturated ring of IPDI were seen in the $2800-3000 \mathrm{~cm}^{-1}$ range in the 100P-TMHDI and 100P-IPDI compositions respectively [162].

The trends of relative FTIR absorbance were as expected. Increase in the PCL-t content of the foam $(0 \mathrm{P} / 25 \mathrm{P} / 50 \mathrm{P} / 100 \mathrm{P}-\mathrm{HDI})$ results in an increased ester content of the material. This was adequately reflected in the successive increase of ester carbonyl $(\mathrm{C}=\mathrm{O})$, and the asymmetric and symmetric ester ether, $v(\mathrm{C}-\mathrm{O}-\mathrm{C})$, band absorbance in 
these compositions. Further, as the compositions with different isocyanate type (100PHDI/TMHDI/IPDI) exclusively used PCL-t as the hydroxyl monomer, no difference in the relative ester and urethane absorbance was observed within these compositions; however, expected variation in the signature isocyanate fingerprints could be identified in the alkyl absorbance band.

\subsection{Shape Memory Behavior}

Shape memory behavior of the 100P-HDI foam samples is reported in Figure 45. Excellent shape recovery of up to $99 \%$ was noticed for all five samples tested. The results obtained were similar to those obtained in a previous study of related compositions [30], i.e. the shape recovery was relatively lower for initial cycles, and was seen to improve for successive cycles. An average shape recovery of $91 \pm 3 \%, 94 \pm 1 \%$, $96 \pm 1 \%, 98 \pm 1 \%$, and $99 \pm 1 \%$ respectively was recorded for the five consecutive cycles over five samples. The loss in shape recovery in the initial few cycles is often attributed to macroscopic changes in the material, such as damage to cell struts/membranes [30]. However, on storage at $T>T_{g}\left(20^{\circ} \mathrm{C}\right)$ for $\sim 24$ hours after the cyclic testing, the samples showed a consistent recovery to $98-99 \%$ of their original dimensions. Hence a significant part of the otherwise minor loss in recovery in the initial cycles was eventually recovered. 

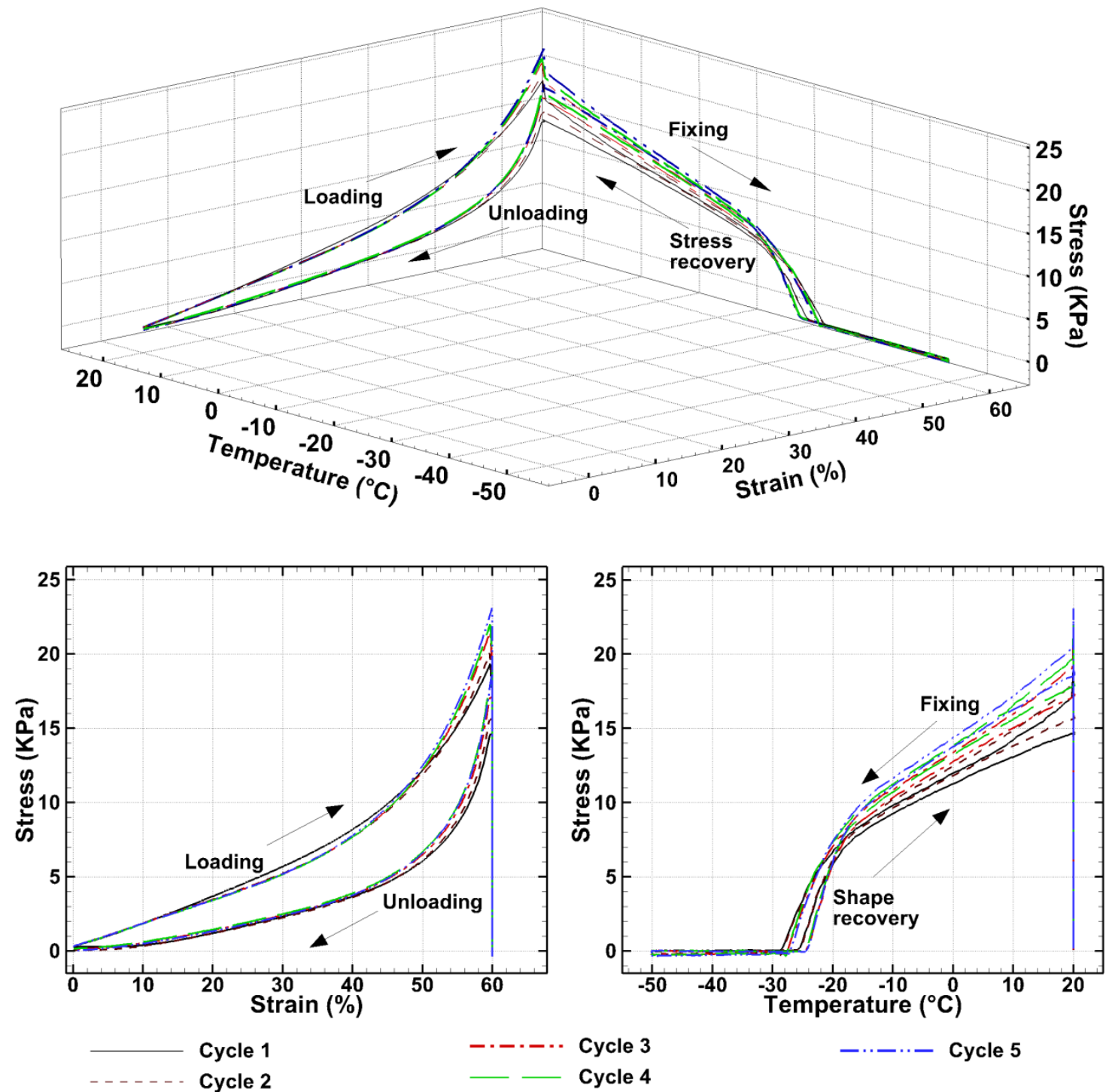

Figure 45: Constrained stress recovery shape memory tests of the 100P-HDI foam samples. The results for five cycles, representing the variation in sample stress with changes in strain and temperature during the loading, fixing, stress recovery and unloading steps, are shown in 3D (top) and 2D (bottom) formats. 


\section{Characterization of Degradation Behavior}

\subsection{Mass Loss}

The mass loss profile of the various foam compositions in $0.1 \mathrm{~N} \mathrm{NaOH}$ is presented in Figure 46. Both reducing the PCL-t content and increasing the hydrophobicity of the isocyanate monomer were seen to significantly reduce the rate of mass loss. The 100P-HDI composition was observed to have the highest mass loss rate. It underwent rapid degradation, and the sample was irretrievable post week 6. Fragments of the disintegrated polymer could be seen in the degradation media at week 7 . These fragments gradually decreased in size and eventually reached the critical chain length for aqueous solubility, thereby undergoing complete dissolution in the media. The 100P-TMHDI sample demonstrated a relatively slower mass loss, and the sample did not disintegrate completely until week 15 . But eventually complete dissolution of the polymer fragments was observed in the media, similar to those of the 100P-HDI composition. 100P-IPDI showed a still slower degradation rate, and maintained its structural integrity through the entire test duration of 7 months. The increase in material hydrophobicity with use of TMHDI and IPDI is expected to be responsible for the reduced mass loss rate in these compositions. 

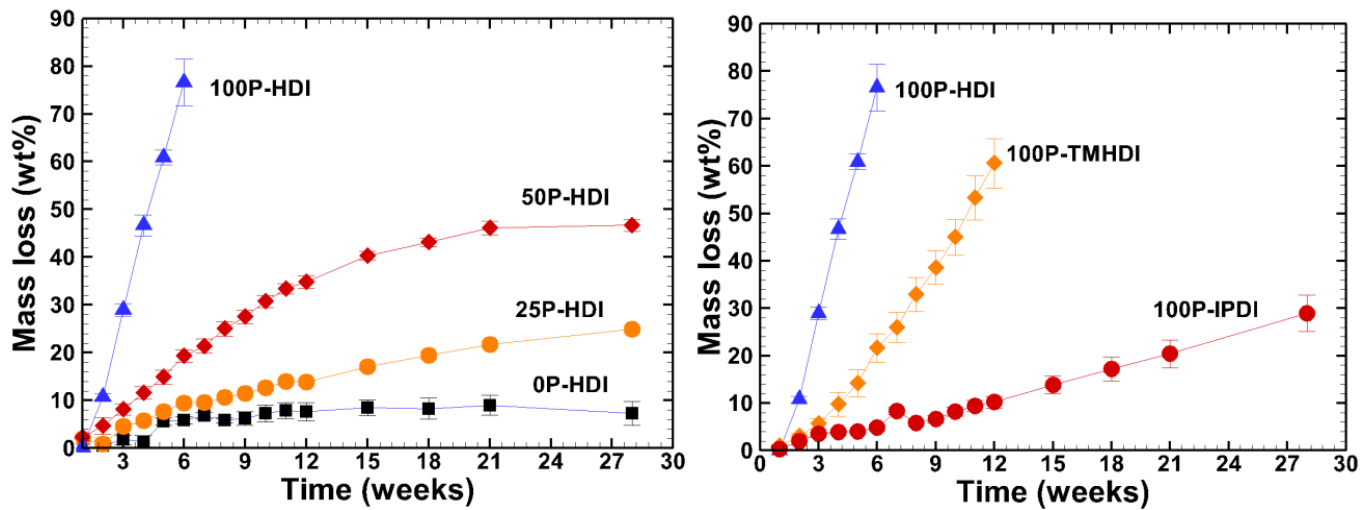

Figure 46: Mass loss profile of the different foam compositions over the first 28 weeks in $0.1 \mathrm{~N} \mathrm{NaOH}$ media. The fastest mass loss is seen in the 100P-HDI composition, which has the highest PCL-t content and the most hydrophilic isocyanate monomer. The rate of mass loss is observed to decrease with both a decreasing PCL-t content and increasing hydrophobicity of the isocyanate monomer.

The rate of mass loss for both 50P-HDI and 25P-HDI compositions was also successively lower relative to that of 100P-HDI, and they did not disintegrate during the test period. This rate is expected to be limited by the diffusion of the degraded molecular fragments from the polymer. As the content of PCL-t was lower in these compositions, the average molecule size diffusing out is expected to be larger, leading to a lower rate of mass loss. Also, with a lower concentration of degradable linkages, the probability for all the branches of these crosslinked materials to get lysed and be eroded from the network is expected to decrease, again lowering the rate of mass loss. No significant mass loss was noticed for the OP-HDI in $0.1 \mathrm{~N} \mathrm{NaOH}$, or for any composition in the PBS media during the 7 month test period. 


\subsection{FTIR}

FTIR spectra for various compositions during the first 12 weeks of degradation are shown in Figure 47. The 100P-HDI composition registered a small decrease in the absorbance of ester carbonyl at $1730 \mathrm{~cm}^{-1}$ in the first week. This absorbance was further reduced in the $2^{\text {nd }}$ week of degradation, and was negligible by the $3^{\text {rd }}$ week. Concomitant reduction in the urethane carbonyl at $1395-1370 \mathrm{~cm}^{-1}$ was also observed. Hydrolysis of the urethane bond is likely not the primary cause of this reduction given that the adjacent ester bonds are more susceptible to hydrolysis. Therefore, this loss of absorbance was attributed to solubilization of low molecular weight polymer segments containing urethane linkages after hydrolysis of the adjacent ester. The rapid mass loss prevented

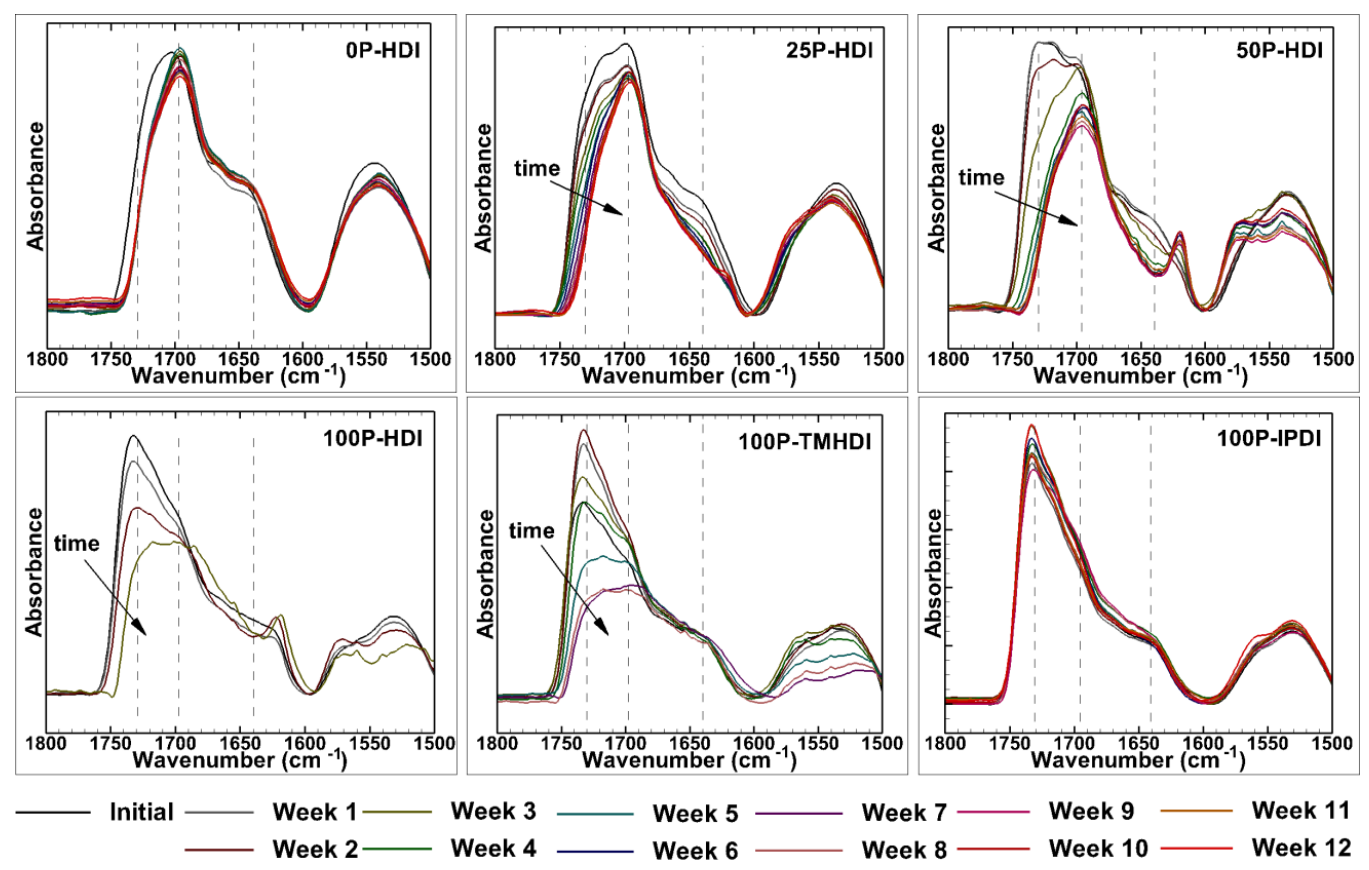

Figure 47: FTIR spectra for all the different compositions are shown as a function of time. The wavenumbers of interest, i.e. ester $\left(1730 \mathrm{~cm}^{-1}\right)$, urethane $\left(1695 \mathrm{~cm}^{-1}\right)$, and urea $\left(1640 \mathrm{~cm}^{-1}\right)$ are marked with dotted lines. 
collection of FTIR spectra after week 4, and the sample was irretrievable post week 6 . The FTIR spectra of the 100P-TMHDI composition followed the same pattern as that of 100P-HDI (Figure 47), but the rate of degradation was slower. No significant change was noticed in the spectrum despite the mass loss until week 5. This suggests the dominance of surface degradation during the initial 5 weeks. Specimens incubated for 6 weeks displayed a loss of absorbance of ester carbonyl at $1730 \mathrm{~cm}^{-1}$, indicative of initiation of bulk degradation at this stage. The sample was finally irretrievable at week 15. No significant variation was observed in the FTIR spectra of 100P-IPDI during the 12 weeks (Figure 47), despite the fixed pattern of mass loss. This may again indicate presence of primarily a surface degradation mechanism, rather than bulk erosion. The transition from bulk erosion in 100P-HDI to the primarily surface degradation mechanism of 100P-TMHDI and 100P-IPDI was attributed to the increased hydrophobicity of these polymers as compared to HDI. This increase in hydrophobicity is expected to limit access of the aqueous degradation media to the ester linkages in the bulk of the sample.

FTIR spectra of the 50P-HDI are also shown in Figure 47. Similar to the 100P-HDI spectra, a decrease in the ester absorbance at $1730 \mathrm{~cm}^{-1}$ is observed, indicating attack of the media throughout the entire bulk of the material. However, a relatively lower rate of decrease in ester absorbance is noticed, compared to that of 100P-HDI. Also, an increase in the absorbance of urea $\left(\sim 1620-1650 \mathrm{~cm}^{-1}\right)$ was noticed over time, indicating the relative decrease in the absorbance of urethane bond. A similar degradation pattern was again observed in 25P-HDI composition (Figure 47) with a still 
lower rate of decrease in ester absorbance. The trend of decrease in the ester absorbance of these compositions is in agreement with the pattern of mass loss, and the decreasing content of PCL-t monomer containing the hydrolysable ester groups. Finally, no statistically significant change in the FTIR spectra was noticed for the OP-HDI composition (control) throughout the degradation period, which is again in agreement with its negligible mass loss.

\subsection{Gross Morphology}

Gross morphology of the fastest degrading 100P-HDI composition at the weekly time points is shown in Figure 48. The sample did not develop cracks or fracture lines during the test period. The material degradation was relatively uniform throughout the sample, and may be attributed to the completely amorphous polymer structure, high hydrophilicity, and the morphological properties of low density and uniform cell structure. This indicates a bulk degradation mechanism for this composition, in agreement with the FTIR results. 


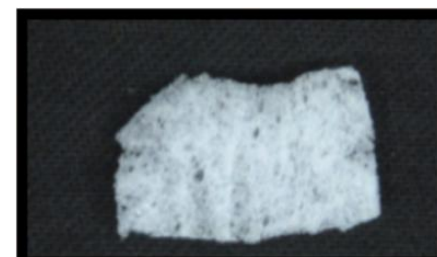

Original

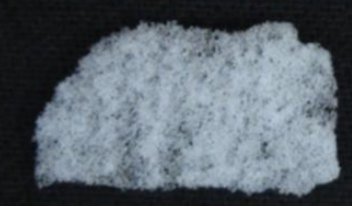

Week 3

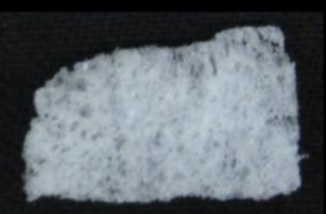

Week 1

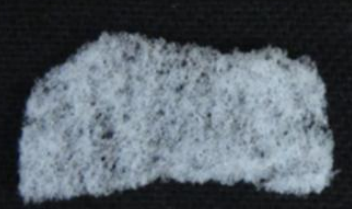

Week 4

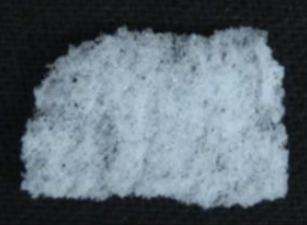

Week 2

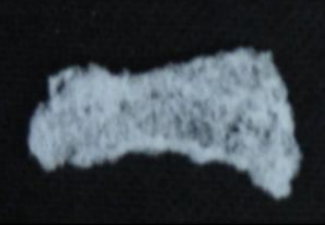

Week 5

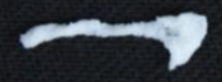

$1 \mathrm{~cm}$

Week 6

Figure 48: Gross morphology of a 100P-HDI composition sample as it degraded over the first 6 weeks. Uniform degradation was observed to occur throughout the bulk of the material, leading to a continuous thinning of the sample, until it became irretrievable at week 7 .

Several biodegradable SMPs have been reported in the literature [194]. A recent review by Behl et al. reports biodegradable SMPs developed in the four primary SMP network structure categories of covalently crosslinked crystalline and amorphous networks, and physically crosslinked crystalline and amorphous networks [194]. Multiple PCL based physically and covalently crosslinked crystalline biodegradable polymers have also been reported [194]. Particularly, covalently crosslinked amorphous biodegradable SMP networks, as proposed in this work, have been reported from coupling star-shaped hydroxyl- telechelic polyesters with a diisocyanate [195]. In this 
polymeric system, star shaped precursors were developed from reaction of polyfunctional initiators such as 1, 1, 1 - tris (hydroxymethyl)ethane and pentaerythrite with copolyester segments from diglycolide or other cyclic diesters and dilactide. Incorporation of a second immiscible phase, such as poly (propylene glycol), in varying content and molecular weight allowed precise control over the mechanical properties of this family of polymers. Another covalently crosslinked amorphous polymeric system was derived from UV polymerization of poly[(l-lactide)-ran-glycolide] dimethaycrylates (PLGDMA), which in turn were obtained from methacryloyl chloride based functionalization of hydroxy telechelic poly[(l-lactide)-ran-glycolide] (PLG) $\left(1000<\mathrm{M}_{\mathrm{n}}<5700 \mathrm{~g} \cdot \mathrm{mol}^{-1}\right)$ [196]. Also, a photo crosslinked linear ABA triblock precursor poly(rac-lactide)-b-poly(propylene oxide)-b poly(rac lactide) dimethacrylate was reported, where $M_{w}$ of rac-lactide was varied from 2000 to $6000 \mathrm{~g} \cdot \mathrm{mol}^{-1}$ in conjunction with a $4000 \mathrm{~g} \cdot \mathrm{mol}^{-1}$ block of polypropylene glycol to control the mechanical properties of the material [197]. Of these materials, the polyurethanes from the starshaped hydroxyl telechelic polyesters of $\mathrm{M}_{\mathrm{w}} \sim 870-1000 \mathrm{~g}$ have a polymer architecture closest to that of the materials reported here, but these were developed in their neat/nonporous form.

To our knowledge, there is limited availability of amorphous biodegradable SMPs that simultaneously show a highly covalently crosslinked polymer structure and a low density porous morphology, as desired for embolic applications [30]. Related compositions of PCL-t $\left(M_{n} 900 \mathrm{~g}\right)$ with HDI have been reported by David et al. using the HIPE process; however, the foams were relatively dense with a non-uniform cell 
structure [125]. The use of the gas blowing process for these compositions, as reported here, not only enabled reasonably low densities $\left(0.020-0.093 \mathrm{~g} \cdot \mathrm{cm}^{-3}\right)$, but also gave a very uniform cell morphology. In part, this is a result of optimization of the DCI990 and DC5179 surfactants to better stabilize the foam structure in the presence of the relatively high molecular weight PCL-t (900 g). Also the viscosity of the foaming solution was adjusted across different compositions by changing the $\mathrm{OH} / \mathrm{NCO}$ ratio of the isocyanate premix to assist in the foam rise. Additional optimization of the foaming solution viscosity and the type and level of the surfactants and additives during the foaming process [30] may however still be conducted to further control the foam porosity/density and cell structure, while maintaining the shape memory properties.

Degradation rate of the materials was observed to be lower for lower PCL-t content, as well as for more hydrophobic, 100P-TMHDI and 100P-IPDI, compositions. A large portion of the synthetic biomaterials used in resorbable devices utilize hydrolytically labile groups to impart a biodegradable character. The primary steps in the hydrolytic degradation process, employed by these materials, have been identified as a) diffusion of the water in the polymer matrix, b) cleavage of the bonds susceptible to hydrolysis, resulting in low molecular weight chains or oligomers, and c) diffusion of these cleaved chains out of the polymer resulting in mass loss [198]. Consequently, the rate of mass loss or degradation can be altered by: 1) water uptake, which is influenced by the polymer composition; or 2) the rate of hydrolysis, which is influenced by the type of chemical bond and the degradation media [198]. It is expected that the use of more hydrophobic TMHDI and IPDI monomers would decrease the water uptake of the 
polymers relative to HDI, thereby affecting the rate of cleavage of hydrolytic ester bonds. Indeed, surface degradation, as opposed to bulk degradation, was observed to be more dominant in the materials as the hydrophobicity was increased. Use of a lower amount of PCL-t content made it slower for a cleaved oligomer to readily diffuse out, again leading to a lower rate of mass loss. However, these materials showed a more bulk degradation effect due to their relatively hydrophilic nature from the use of HDI. This degradation study was accelerated via use of a $\mathrm{pH} 13$ media ( $0.1 \mathrm{M} \mathrm{NaOH}$ solution), in order to achieve measurable rates in shorter time frames. No statistically significant mass loss was recorded for samples in the physiological PBS degradation media over the duration of this study. But as crystalline PCL has been shown to undergo degradation in 2-3 years in a physiological medium [193], it is expected that the 100P-HDI composition would degrade earlier than that due to its amorphous state. In vivo implantation of these materials will be subject to enzymatic degradation as well, which may further increase the degradation rate. The aliphatic monomers used in these compositions are expected to release biocompatible degradation products. However, animal studies or cell culture tests will need to be performed to fully ascertain the material biocompatibility.

A decrease in the $T_{g}$ of the material was observed with a decrease in hydrophobicity and increase in the PCL-t content. For polyurethane materials, the asmeasured dry $T_{g}$ has been reported to get further depressed on exposure to water/physiological media [29]. Hence, for use of these materials for intravascular aneurysm embolization, it is essential that the depressed/plasticized $T_{g}$ in fluid media is above body temperature $\left(37^{\circ} \mathrm{C}\right)$ to prevent premature actuation of the device during 
transcatheter delivery. Based on the trends observed here, a $T_{g}$ of $35-40{ }^{\circ} \mathrm{C}$ may be achieved by substituting part of PCL-t with HPED or TEA in the 100P-IPDI composition, while still maintaining degradability of the material. Given that IPDI is relatively hydrophobic, further depression in $T_{g}$ in presence of water/physiological media may also not be as significant, facilitating the in vivo transcatheter delivery of the device [29]. It is noteworthy though, that because the rate of degradation of materials is directly affected by the uptake of water, it may not be possible to achieve a material with a high degradation rate, as well as a low degree of plasticization, in water/physiological media. Hence, it is suggested that a different strategy, such as surface modification, may be employed to limit the plasticization rate during delivery of the device made from an otherwise hydrophilic/faster degrading material.

\section{Conclusions}

A highly covalently crosslinked polymer network structure was maintained by use of low molecular weight, symmetrical and branched hydroxyl monomers such as PCL-t ( $\left.\mathrm{M}_{\mathrm{n}} 900 \mathrm{~g}\right)$, HPED, and TEA. A control over the degradation rate of the materials was achieved by changing the concentration of the degradable PCL-t monomer in the foam formulation, and also by successively increasing the hydrophobicity of the isocyanate monomers going from HDI to TMHDI and IPDI respectively. A uniform and fine cell structure was obtained for all compositions. In addition, excellent shape recovery of $\sim 98-99 \%$, and a tunable $T_{g}$ in the range of -19 to $29{ }^{\circ} \mathrm{C}$ was achieved. Degradation of the samples was monitored for a period of 7 months in $0.1 \mathrm{~N} \mathrm{NaOH}$, as 
well as phosphate buffer saline media. An increase in the degradation rate of the foams was observed for both an increase in the PCL-t content and a decrease in the isocyanate hydrophobicity. We believe that these novel materials form a new class of low density biodegradable SMP scaffolds that can potentially be used as "smart" non-permanent implants in multiple minimally invasive biomedical applications. 


\section{CHAPTER VI}

\section{CONCLUSIONS AND FUTURE WORK}

This dissertation aimed at developing novel shape memory polymer scaffolds customized for the embolic biomedical application of treatment of intracranial aneurysms. Work was performed to first fabricate the material, based on the hypothesis that a highly covalently crosslinked polymer structure would enable good mechanical properties at the desired ultra low bulk densities, using a gas blowing process optimized for achieving ultra low densities. Thereafter, characterization of the physico-mechanical properties of foams was performed to understand the effect of underlying polymer structure on the material properties, and the results were compared to other commercially available/published SMP foams. However, when further testing of these materials was done in an in vitro set up to estimate its efficacy in the proposed biomedical device, a problem of pre-mature actuation of foam was observed. Consequently changes were made to the chemical composition of the foams to avoid this issue. Successful tests were thereafter conducted with the modified materials. Beyond these essential "need to have" properties, some other desirable material modifications were made to achieve a) extremely low density values for improved device efficiency, and b) completely reticulated foam structure for controlled fluid permeability. Finally, controlled biodegradability was introduced in these materials for complete long term healing of the vasculature. The results obtained can be categorized in four sections that are summarized below. 


\section{A. Fabrication of Ultra Low Density Foams}

Novel polyurethane shape memory polymer foams were synthesized based on small molecular weight polyfunctional monomers to get a highly covalently crosslinked network structure with low molecular weight between crosslinks. Based on the classical equation of $E=n_{c} R T$, it has been estimated that low molecular weight segments between chemical crosslinks would give a high modulus, thereby leading to good mechanical properties at the ultra low material densities. A gas blowing process, optimized for this polymeric system, was employed for foam fabrication. Generation of a continuous, highly crosslinked structure was confirmed using the swelling and extraction studies which demonstrated that $\sim 96-99 \%$ of mass was incorporated in the network. Furthermore, extensive hydrogen bonding was deduced from the FTIR results confirming the generation of a highly covalently crosslinked structure.

With regards to the utility of this material in the proposed device, the actuation temperature of the foams could be customized in the highly functional range of $45-70{ }^{\circ} \mathrm{C}$ by controlled variation in the ratio of constituent monomers. Ultra low densities $(\sim 0.015-$

$0.021 \mathrm{~g} \cdot \mathrm{cm}^{-3}$ ) were achieved, with a very uniform cell structure across the bulk of the foam; however, they were observed to have a mixed, closed to open cell morphology, with thin residual cell membranes on the cell surfaces. As expected from the polymer design, a high glassy storage modulus of 200-300 kPa was recorded despite such low densities, and an excellent shape recovery of $97-98 \%$ was achieved post initial conditioning cycles. Up to a 70 times volume expansion was recorded for these materials, which was a significant improvement over other known SMP foams. In 
addition, encouraging biocompatibility results were obtained on the basis of cytokine production by human blood cells in vitro.

As another important accomplishment in this work, the drawback of secondary-shape forming above $T_{g}$ seen in the traditional physically crosslinked foams was significantly reduced through a high density of covalent crosslinks in the structure. Shape recovery of $94 \%$ (for $T_{g} \sim 50{ }^{\circ} \mathrm{C}$ ) and $\sim 95 \%$ (for $T_{g} \sim 60{ }^{\circ} \mathrm{C}$ ) was achieved for these foams in contrast to $\sim 0 \%$ shape recovery of physically crosslinked foams $\left(T_{g} \sim 60{ }^{\circ} \mathrm{C}\right)$ under similar storage conditions (80\% compressive strain at $T_{g}+60{ }^{\circ} \mathrm{C}$ for 2 hours). Although conditions of higher temperatures, longer holding times and larger strains were not investigated in detail here, this result suggests an improved shelf life of these foams for storage in a compressed secondary shape below $T_{g}$.
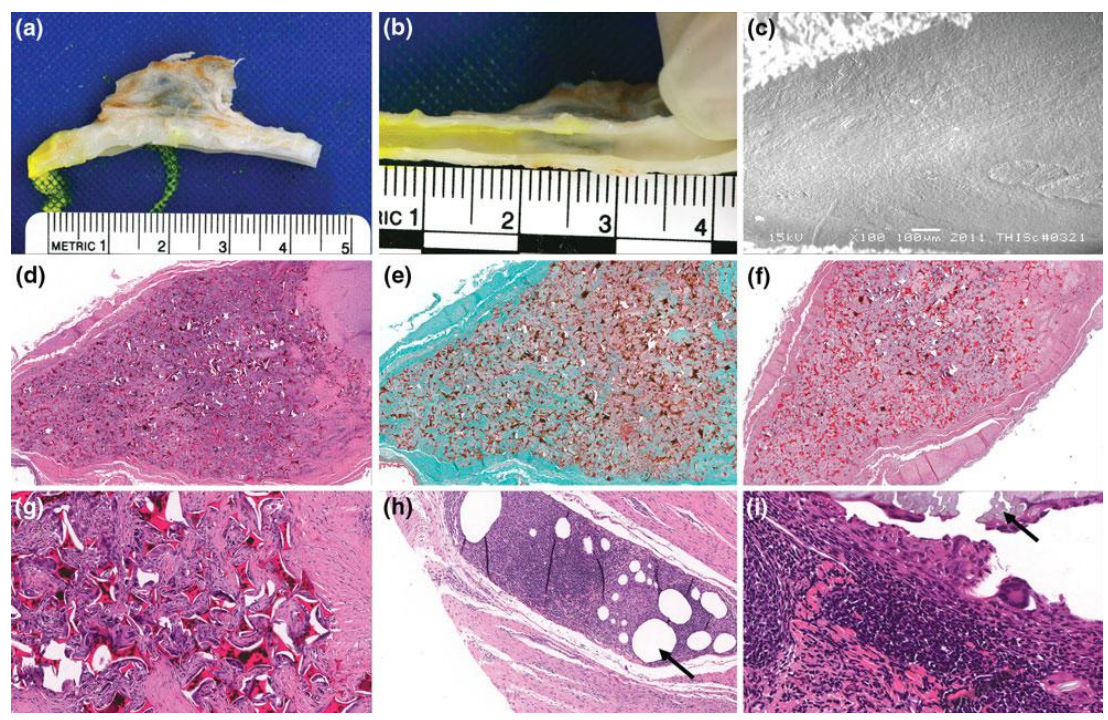

Figure 49: SEM and pathology results of implanted foams demonstrated very promising biocompatibility of the foams. Reproduced with permission from [54]. 
These polyurethane foams, to our knowledge, are unique in the aspect that they simultaneously show a very highly crosslinked polymeric structure and very low material densities down to $0.015 \mathrm{~g} \cdot \mathrm{cm}^{-3}$. These exceptional properties, in addition to the encouraging in vitro and in vivo [54] biocompatibility results (Figure 49), suggest that these foams are particularly promising as biomaterials for embolic devices in minimally invasive medical applications.

\section{B. Controlled Variation in the Material Hydrophilicity}

The material characterization results were very encouraging. However, initial in vitro testing of the embolic devices based on these materials revealed a significant problem: It was found that the material's actuation temperature got depressed to values much below the body temperature of $37^{\circ} \mathrm{C}\left(\sim 5-12{ }^{\circ} \mathrm{C}\right)$ on their exposure to water. This meant that as the device is inserted into the catheter for delivery to the aneurysm site, it would come in contact with body temperature saline/physiological media, and would undergo premature actuation, thereby hindering the delivery process. Other studies describing similar observation with polyurethane SMPs attributed this behavior to the disruption of $\mathrm{N}-\mathrm{H}$ and $\mathrm{C}=\mathrm{O}$ hydrogen bonding in the polymer by water molecules, which thereby led to an increase in the mobility of polymer chains and depressed the polymer $T_{g}$

To address this issue, a series of low density, highly covalently crosslinked SMP foams was synthesized with varying hydrophobicity by substituting HDI with a more hydrophobic TMHDI in the foam composition, (0TM through 100TM). Contact angle 
and water uptake measurements confirmed that the hydrophobicity of these materials could be controllably increased via increase in the TMHDI content, consistent with the theoretical solubility parameter values of these compositions. Also, the depressed $T_{g}$ could be controlled from $\sim 12{ }^{\circ} \mathrm{C}$ (for $0 \mathrm{TM}$ foams) to $\sim 40{ }^{\circ} \mathrm{C}$ (for $100 \mathrm{TM}$ foams) after submersion in water. A corresponding reduction in the foam actuation rate was observed, and time of complete actuation of a compressed foam device could be controlled from within 2 minutes (for 0TM foams) to up to more than 24 hours (for 100TM foams). Such control over the actuation rate of SMP foams by virtue of their composition has, to our knowledge, not been reported before. This provides the basis of a polymer synthesis toolbox that, we expect, will be useful in the development of passively or actively actuating, embolic/regenerative porous SMP scaffolds for biomedical applications.

\section{Further Modification of the Foams}

In the proposed treatment of the neurovascular aneurysm, only a 1-2 Fr microcatheter with typical lumen diameter of less than $1 \mathrm{~mm}$ can potentially be used. This limited space is, however, required to deliver a device that can fill an aneurysm of $\sim 8 \mathrm{~mm}$ diameter. Without doubt, this engineering feat demanded materials with extremely high volume expansion on actuation, beyond what had been achieved until now. Further, an improved control over the cell morphology was desired by achieving a means to get rid of the residual cell membranes in a regulated manner. These objectives were achieved as follows: 


\section{Still Lower Densities}

Foams of significantly lower densities of down to $0.008 \mathrm{~g} \cdot \mathrm{cm}^{-3}$ were achieved through a series of additive and process modifications. These modifications involved a) optimization of surfactants: a more water soluble DC5943 was used and empirically optimized for the appropriate addition levels, b) use of higher level of blowing agent: a $133 \%$ increment was made in the blowing agent levels that led to increased generation of the gaseous phase for lower densities, c) use of a larger mold: a 2.3 times increase in the mold size allowed more space for lateral expansion of the foam, and finally d) application of slow vacuum during foaming: this assisted in the foam rise and stability by opposing the effect of gravity and atmospheric pressure on the expanding foam. These modifications not only helped in reproducible densities of down to $0.008 \mathrm{~g} \cdot \mathrm{cm}^{-3}$, but also led to significant improvement in the structural uniformity and dimensional stability of the foams. These advancements were very promising for significant increase in the embolic efficiency of the device.

\section{Complete Reticulation}

Complete reticulation of the foams (removal of the residual cell membranes) was achieved using Plasma treatment. In this process, a $\mathrm{O}_{2} / \mathrm{CF}_{4}$ atmosphere was employed under low electrode power to achieve controlled oxidation of the cell membranes. This process also led to a slight increase in the actuation temperature $\left(\sim 5^{\circ} \mathrm{C}\right)$, and decrease in the modulus by a factor of $\sim 2$. But the shape memory behavior was seen to improve significantly, showing little hysteresis in the initial compression-expansion cycles. Close 
to a $99 \%$ recovery was seen in all cycles of shape memory, and lack of residual cell membranes was thought to be critical to this result. In general, this state-of-the-art plasma process proved to be a highly effective and simple way for membrane removal of the foams. This was a very desirable outcome as it gives us a means to precisely control the permeability of the foams during delivery and implantation, possibly with controlled variations to the in vivo physiological response to the foams.

\section{Biodegradable Foams}

A series of novel biodegradable shape memory polymer foams were synthesized for the proposed embolic biomedical applications, to achieve complete long term healing of the vasculature. A highly covalently crosslinked polymer network structure was maintained by use of the degradable trifunctional monomer PCL-t $\left(M_{n} 900 \mathrm{~g}\right)$, and the tri- and tetra- functional monomers TEA and HPED, respectively. Multiple formulations were prepared from successively increasing the PCL-t content, and from substituting HDI with more hydrophobic TMHDI and IPDI. Very low densities of 0.020 to 0.093 $\mathrm{g} \cdot \mathrm{cm}^{-3}$ were achieved for these foams. A uniform cell structure was observed across all compositions, and a high shape recovery ( $98-99 \%)$ was recorded. The $T_{g}$ was observed to decrease with an increase in the content of the degradable monomer PCL-t. Also, an increase in the $T_{g}$ was recorded with the increase in the hydrophobicity of the material via incorporation of more hydrophobic monomers, TMHDI and IPDI, in lieu of HDI. A controlled $T_{g}$ variation from $-19^{\circ} \mathrm{C}$ to $29^{\circ} \mathrm{C}$ was thereby achieved. 
The degradation rate was found to be depended on the PCL-t content, as well as the hydrophobicity of the composition. The composition with the highest PCL-t content and lowest hydrophobicity (100P-HDI) achieved complete degradation in $0.1 \mathrm{~N} \mathrm{NaOH}$ in 5 weeks. On the other hand, replacing the HDI with more hydrophobic monomers or reducing the PCL-t content, greatly reduced the degradation rate with 100P-IPDI and 25P-HDI showing only $\sim 29 \%$ and $25 \%$ mass loss respectively at 28 weeks. FTIR analysis of the degrading samples showed an increasing prominence of surface degradation as opposed to bulk degradation as the hydrophobicity of the material was increased by incorporation of TMHDI and IPDI.

The excellent morphological and shape memory properties in conjunction with the control over degradation rate of these biodegradable foams suggested their utility in multiple non-permanent minimally invasive implants comprising low density smart scaffolds.

\section{E. Future Directions}

This dissertation laid the groundwork for establishing the methods for fabrication and processing of ultra low density SMP foams, and understanding the effect of underlying molecular structure on the physico-mechanical properties of these novel polymeric systems. Further, it demonstrated the feasibility of their use in an embolic medical device; a device based on a combination of the 0TM $\left(0.020 \mathrm{~g} \cdot \mathrm{cm}^{-3}\right)$ foam, and

the much lower density $\left(0.010 \mathrm{~g} \cdot \mathrm{cm}^{-3}\right)$ 80TM composition foams has been successfully implanted in a porcine model using a transcatheter procedure, and is currently 
undergoing animal trials for longer term (3 to 9 months) biocompatibility. Going forward, the main emphasis is initially expected to be towards translating the understanding achieved from the studies of plasma reticulation process, and biodegradable foams, into clinically relevant devices.

With respect to reticulation, the various process variables of plasma treatment procedure need to be optimization for a given material. This may be geared towards achieving complete reticulation throughout the sample, or a graded reticulation as a function of sample depth, in order to modulate the permeability of the device in physiological fluid/blood. Optimization may also be necessary as the material composition is changed. In essence, a complete understanding of a) the mechanisms of interaction of the plasma and foams, and b) the effect of the parameters such as gas composition, temperature, gas concentration, applied voltage, sample size, and sample positioning, needs to be developed.

With respect to the biodegradable materials, the current work gives us a detailed understanding of the trends of actuation temperatures, degradation rates, and the degradation mechanisms of the foams, with changes in the material composition. For the final biomedical device, further optimization of the foaming additives will need to be performed for the material composition with preferred characteristics of actuation temperature and degradation behavior, to achieve densities in the desirable range. Another direction is to generate the degradation rates in a purely PBS media, with or without physiologically relevant enzymes, to get an estimate of the in vivo degradation period of these devices after implantation. While experimental determination of these 
degradation periods may take a long time, time-temperature superposition techniques can provide relevant data within a more favorable time frame.

Overall, this family of ultra low density biomedical scaffolds presents a highly promising avenue for the future of minimally invasive implantable embolic/regenerative devices, and forms an exciting area for continued research. 


\section{REFERENCES}

[1] Liu C, Qin H, Mather P. Review of Progress in Shape-Memory Polymers. Journal of Materials Chemistry 2007; 17:1543-58.

[2] Lendlein A, Kelch S. Shape-Memory Polymers. Angewandte Chemie International Edition 2002; 41:2034-57.

[3] Mather PT, Luo X, Rousseau IA. Shape Memory Polymer Research. Annual Review of Materials Research 2009; 39:445-71.

[4] Rousseau IA. Challenges of Shape Memory Polymers: A Review of the Progress Toward Overcoming SMP's Limitations. Polymer Engineering and Science 2008; 48:2075-89.

[5] Behl M, Lendlein A. Shape-Memory Polymers. Materials Today 2007; 10:20-8.

[6] Metcalfe A, Desfaits AC, Salazkin I, Yahia LH, Sokolowski WM, Raymond J. Cold Hibernated Elastic Memory Foams for Endovascular Interventions. Biomaterials 2003; 24:491-7.

[7] Sokolowski WM, Hayashi S. Applications of Cold Hibernated Elastic Memory (CHEM) Structures. In: Proceedings of the SPIE Smart Structures and Materials: Smart Structures and Integrated Systems in San Diego (USA). 2003. edited by Baz AM, 5056:534-44.

[8] Small IV W, Singhal P, Wilson TS, Maitland DJ. Biomedical Applications of Thermally Activated Shape Memory Polymers. Journal of Materials Chemistry 2010; 20:3356-66.

[9] Ratna D, Karger-Kocsis J. Recent Advances in Shape Memory Polymers and Composites: A Review. Journal of Materials Science 2008; 43:254-69.

[10] Lendlein A, Behl M, Hiebl B, Wischke C. Shape-Memory Polymers as a Technology Platform for Biomedical Applications. Expert Review of Medical Devices 2010; 7:357-79. 
[11] Vernon LB, Vernon HM. Process of Manufacturing Articles of Thermoplastic Synthetic Resins. US Pat., 2234993 The Vernon-Benshoff Company, Pittsburg, Pa 1941.

[12] Rainer WC, Redding EM, Hitov JJ, Sloan AW, Stewart WD. Heat-shrinkable Polyethylene. US Pat., 31443981964.

[13] Perrone RJ. Heat-shrinkable Articles made from Silicone Rubber-Polyethylene Compositions. US Pat., 33268691967.

[14] Wray PE. Elastic Memory Articles. US Pat., GB1075704 Raychem Corp. 1967.

[15] Arditti SJ, Avedikian SZ, Bernstein BS. Articles with Polymeric Memory and Method of Constructing the Same. US Pat., 3563973 Radiation Applications Inc. 1971.

[16] Behl M, Razzaq MY, Lendlein A. Multifunctional Shape Memory Polymers. Advanced Materials 2010; 22:3388-410.

[17] Lendlein A, Jiang H, Junger O, Langer R. Light-induced Shape Memory Polymers. Nature 2005; 434:879-82.

[18] Han XJ, Dong ZQ, Fan MM, Liu Y, Li JH, Wang YF, Yuan QJ, Li BJ, Zhang S. pH-induced Shape-Memory Polymers. Macromolecular Rapid Communications 2012; 33:1055-60.

[19] Hearon K, Singhal P, Horn J, Small IV W, Olsovsky C, Maitland KC, Wilson TS, Maitland DJ. Porous Shape Memory Polymers Polymer Reviews 2012; (in press).

[20] Hayashi S, Fujimura H. Shape Memory Polymer Foam. 5,049,591 Mitsubishi Jukogya Kabushiki Kaisha, Tokyo, Japan 1991.

[21] Tobushi H, Matsui R, Hayashi S, Shimada D. The Influence of Shape-holding Conditions on Shape Recovery of Polyurethane-Shape Memory Polymer Foams. Smart Materials and Structures 2004; 13:881-7.

[22] Tobushi H, Okumura K, Endo M, Hayashi S. Thermomechanical Properties of Polyurethane-Shape Memory Polymer Foam. Journal of Intelligent Material Systems and Structures 2001; 12:283-7. 
[23] De Nardo L, Alberti R, Cigada A, Yahia LH, Tanzi MC, Farè S. Shape Memory Polymer Foams for Cerebral Aneurysm Reparation: Effects of Plasma Sterilization on Physical Properties and Cytocompatibility. Acta Biomaterialia 2009; 5:1508-18.

[24] Domeier L, Nissen A, Goods S, Whinnery LR, McElhanon J. Thermomechanical Characterization of Thermoset Urethane Shape Memory Polymer Foams. Journal of Applied Polymer Science 2010; 115:3217-29.

[25] Kang SM, Lee SJ, Kim BK. Shape Memory Polyurethane Foams. eXPRESS Polymer Letters 2012; 6:63-9.

[26] Lee SH, Jang MK, Hee Kim S, Kim BK. Shape Memory Effects of Molded Flexible Polyurethane Foam. Smart Materials and Structures 2007; 16:2486.

[27] Chung SE, Park CH. The Thermoresponsive Shape Memory Characteristics of Polyurethane Foam. Journal of Applied Polymer Science 2010; 117:2265-71.

[28] Madbouly SA, Kratz K, Klein F, Lüetzow K, Lendlein A. Thermomechanical Behaviour of Biodegradable Shape-Memory Polymer Foams. Materials Research Society Proceedings 2009; 1190:1190-NN04-04.

[29] Singhal P, Boyle T, Infanger S, Letts S, Small W, Maitland DJ, Wilson TS. Controlling the Rate of Actuation of Shape Memory Polymer Foams in Water. Macromolecular Chemistry and Physics 2012; (in press) DOI: 10.1002/macp.201200342.

[30] Singhal P, Rodriguez JN, Small W, Eagleston S, Van de Water J, Maitland DJ, Wilson TS. Ultra Low Density and Highly Crosslinked Biocompatible Shape Memory Polyurethane Foams. Journal of Polymer Science Part B: Polymer Physics 2012; 50:724-37.

[31] Li G, Nettles D. Thermomechanical Characterization of a Shape Memory Polymer based Self-repairing Syntactic Foam. Polymer 2010; 51:755-62.

[32] Xu T, Li G, Pang S-S. Effects of Ultraviolet Radiation on Morphology and Thermo-Mechanical Properties of Shape Memory Polymer based Syntactic 
Foam. Composites Part A: Applied Science and Manufacturing 2011; 42:152533.

[33] Xu W, Li G. Thermoviscoplastic Modeling and Testing of Shape Memory Polymer Based Self-Healing Syntactic Foam Programmed at Glassy Temperature. Journal of Applied Mechanics 2011; 78:061017.

[34] Li G, Xu T. Thermomechanical Characterization of Shape Memory Polymer Based Self-Healing Syntactic Foam Sealant for Expansion Joints. Journal of Transportation Engineering 2011; 137:805-14.

[35] Simkevitz S, Naguib HE. Development of Two Part Porous Shape Memory Polymer Nanocomposites. ASME Conference Proceedings 2008; 2008:453-60.

[36] Simkevitz SL, Naguib HE. Fabrication and Analysis of Porous Shape Memory Polymer and Nanocomposites. High Performance Polymers 2010; 22:159-83.

[37] Monkman GJ. Robotic Compliance Control using Memory Foams. Industrial Robot: An International Journal 1991; 18:31-2.

[38] De Nardo L, Moscatelli M, Silvi F, Tanzi M, Yahia LH, Farè S. Chemicophysical Modifications Induced by Plasma and Ozone Sterilizations on Shape Memory Polyurethane Foams. Journal of Materials Science: Materials in Medicine 2010; 21:2067-78.

[39] De Nardo L, Bertoldi S, Tanzi M, Haugen H, Farè S. Shape Memory Polymer Cellular Solid Design for Medical Applications. Smart Materials and Structures 2011; 20:035004.

[40] Tey S, Huang W, Sokolowski W. Influence of Long-term Storage in Cold Hibernation on Strain Recovery and Recovery Stress of Polyurethane Shape Memory Polymer Foam. Smart Materials and Structures 2001; 10:321.

[41] Huang W, Lee C, Teo H. Thermomechanical Behavior of a Polyurethane Shape Memory Polymer Foam. Journal of Intelligent Material Systems and Structures 2006; 17:753-60. 
[42] Vialle G, Di Prima M, Hocking E, Gall K, Garmestani H, Sanderson T, Arzberger SC. Remote Activation of Nanomagnetite Reinforced Shape Memory Polymer Foam. Smart Materials and Structures 2009; 18:115014.

[43] Radford D, Antonio A. Enhancing the Deformation of Shape Memory Sandwich Panels. Strain 2011; 47:534-43.

[44] Di Prima M, Gall K, McDowell DL, Guldberg R, Lin A, Sanderson T, Campbell D, Arzberger SC. Deformation of Epoxy Shape Memory Polymer Foam. Part I: Experiments and Macroscale Constitutive Modeling. Mechanics of Materials 2010; 42:304-14.

[45] Di Prima MA, Gall K, McDowell DL, Guldberg R, Lin A, Sanderson T, Campbell D, Arzberger SC. Deformation of Epoxy Shape Memory Polymer Foam: Part II. Mesoscale Modeling and Simulation. Mechanics of Materials 2010; 42:315-25.

[46] Di Prima MA, Gall K, McDowell DL, Guldberg R, Lin A, Sanderson T, Campbell D, Arzberger SC. Cyclic Compression Behavior of Epoxy Shape Memory Polymer Foam. Mechanics of Materials 2010; 42:405-16.

[47] Li G, John M. A Self-healing Smart Syntactic Foam under Multiple Impacts. Composites Science and Technology 2008; 68:3337-43.

[48] Li G, Uppu N. Shape Memory Polymer based Self-healing Syntactic Foam: 3-D Confined Thermomechanical Characterization. Composites Science and Technology 2010; 70:1419-27.

[49] John M, Li G. Self-healing of Sandwich Structures with a Grid Stiffened Shape Memory Polymer Syntactic Foam Core. Smart Materials and Structures 2010; 19:075013.

[50] Quadrini F, Santo L, Squeo EA. Solid-state Foaming of Nano-Clay-Filled Thermoset Foams with Shape Memory Properties. Polymer-Plastics Technology and Engineering 2012; 51:560-7.

[51] Squeo EA, Quadrini F. Shape Memory Epoxy foams by Solid-state Foaming. Smart Materials and Structures 2010; 19:105002. 
[52] Fabrizio Q, Loredana S, Anna SE. Shape Memory Epoxy Foams for Space Applications. Materials Letters 2012; 69:20-3.

[53] Rodriguez JN, Clubb F, Wilson TS, Miller MW, Fossum T, Hartman J, Tuzun E, Singhal P, Maitland DJ. In vivo Tissue Response Following Implantation of Shape Memory Polyurethane Foam in a Porcine Aneurysm Model. Journal of Biomedical Materials Research Part A 2012:(submitted).

[54] Rodriguez JN, Yu YJ, Miller MW, Wilson TS, Hartman J, Clubb FJ, Gentry B, Maitland DJ. Opacification of Shape Memory Polymer Foam Designed for Treatment of Intracranial Aneurysms. Annals of Biomedical Engineering 2011:115.

[55] Yu YJ, Hearon K, Wilson TS, Maitland DJ. The Effect of Moisture Absorption on the Physical Properties of Polyurethane Shape Memory Polymer Foams. Smart Materials and Structures 2011; 20:085010.

[56] Zhang D, Burkes WL, Schoener CA, Grunlan MA. Porous Inorganic-Organic Shape Memory Polymers. Polymer 2012; 53:2935-41.

[57] Baker RM, Henderson JH, Mather PT. Poly(caprolactone) Shape Memory Scaffold for Bone Tissue Engineering. In: Bioengineering Conference (NEBEC), 2012 38th Annual Northeast 2012. edited by 309-10.

[58] Gong T, Li W, Chen H, Wang L, Shao S, Zhou S. Remotely Actuated Shape Memory Effect of Electrospun Composite Nanofibers. Acta Biomaterialia 2012; 8:1248-59.

[59] Wu X, Liu Y, Leng J. Study on Shape Recovery Speed of SMP, SMP Composite, and SMP Foam. In: Nanosensors and Microsensors for Bio-Systems, 2008. edited by Varadan VK, 6931:69310D1-8.

[60] Hentze HP, Antonietti M. Porous Polymers and Resins for Biotechnological and Biomedical Applications. Reviews in Molecular Biotechnology 2002; 90:27-53.

[61] Yakacki C, Gall K. Shape-Memory Polymers for Biomedical Applications. In: Lendlein A, editor. Shape-Memory Polymers: Springer Berlin Heidelberg; 2010. p. $147-75$. 
[62] El Feninat F, Laroche G, Fiset M, Mantovani D. Shape Memory Materials for Biomedical Applications. Advanced Engineering Materials 2002; 4:91-104.

[63] Hwang W, Volk B, Akberali F, Singhal P, Criscione J, Maitland D. Estimation of Aneurysm Wall Stresses Created by Treatment with a Shape Memory Polymer Foam Device. Biomechanics and Modeling in Mechanobiology 2012; 11:715-29.

[64] Small IV W, Gjersing E, Herberg JL, Wilson TS, Maitland DJ. Magnetic Resonance Flow Velocity and Temperature Mapping of a Shape Memory Polymer Foam Device. Biomedical Engineering Online 2009; 8:42.

[65] Ortega J, Maitland D, Wilson T, Tsai W, Savaş Ö, Saloner D. Vascular Dynamics of a Shape Memory Polymer Foam Aneurysm Treatment Technique. Annals of Biomedical Engineering 2007; 35:1870-84.

[66] Small W, Buckley PR, Wilson TS, Benett WJ, Hartman J, Saloner D, Maitland DJ. Shape Memory Polymer Stent with Expandable Foam: A New Concept for Endovascular Embolization of Fusiform Aneurysms. Biomedical Engineering, IEEE Transactions on 2007; 54:1157-60.

[67] Sokolowski WM, Tan SC. Advanced Self-deployable Structures for Space Applications. Journal of Spacecraft and Rockets 2007; 44:750-4.

[68] Sokolowski W, Tan S, Willis P, Pryor M. Shape Memory Self-deployable Structures for Solar Sails. In: Voelcker NH, Thissen HW, editors. Proc SPIE 7267, Smart Materials V, 72670K Melbourne, Australia 2008.

[69] Perkins DA, Reed JL, Havens E. Adaptive Wing Structures. In: Proceedings of SPIE, 5388, Smart Structures and Materials: Industrial and Commercial Applications of Smart Structures Technologies San Diego, CA. 2004. edited by Anderson EH,

[70] Sofla AYN, Meguid SA, Tan KT, Yeo WK. Shape Morphing of Aircraft Wing: Status and Challenges. Materials \& Design 2010; 31:1284-92.

[71] Xu T, Li G. Cyclic Stress-Strain Behavior of Shape Memory Polymer based Syntactic Foam Programmed by 2-D Stress Condition. Polymer 2011; 52:457180. 
[72] Yuan Y, Goodson J, Johnson M, Gerrard D. In-Situ Mechanical and Functional Behavior Characterization of a Shape Memory Polymer for Sand Control Applications. SPE Drilling \& Completion 2012; 27:253-63.

[73] Ozan C, Van der Zee W, Brudy M, Vinson J. Mechanical Modeling of Shape Memory Polyurethane Foam for Application as a Sand Management Solution. SPE Annual Technical Conference and Exhibition Denver, Colorado, USA: Society of Petroleum Engineers 2011. p. 2210-9.

[74] Carrejo N, Horner D, Johnson M. Shape Memory Polymer as a Sand Management Alternative to Gravel Packing. Canadian Unconventional Resources Conference. Alberta, Canada: Society of Petroleum Engineers 2011. p. 408-20.

[75] Carrejo N, Horner D, Johnson M. The Effects of Dynamic Loading on the Sand Management and Permeability of Shape Memory Polymer and Gravel Packs for Sand Management Applications. Brasil Offshore Conference and Exhibition. Macae, Rio de Janeiro: Society of Petroleum Engineers 2011. p. 203-11.

[76] Rinkel GJE, Djibuti M, Algra A, Gijn van J. Prevalence and Risk of Rupture of Intracranial Aneurysms: A Systematic Review. Stroke 1998; 29:251-6.

[77] Viñuela F, Duckwiler G, Mawad M. Guglielmi Detachable Coil Embolization of Acute Intracranial Aneurysm: Perioperative Anatomical and Clinical Outcome in 403 Patients. Journal of Neurosurgery 1997; 86:475-82.

[78] Ferguson GG. Physical Factors in the Initiation, Growth, and Rupture of Human Intracranial Saccular Aneurysms. Journal of Neurosurgery 1972; 37:666-77.

[79] Burns JD, Huston III J, Layton KF, Piepgras DG, Brown Jr RD. Intracranial Aneurysm Enlargement on Serial Magnetic Resonance Angiography: Frequency and Risk Factors. Stroke 2009; 40:406.

[80] Toth M, Nadasy G, Nyary I, Kerenyi T, Orosz M, Molnárka G, Monos E. Sterically Inhomogenous Viscoelastic Behavior of Human Saccular Cerebral Aneurysms. Journal of Vascular Research 2000; 35:345-55. 
[81] Zhang B, Fugleholm K, Day LB, Ye S, Weller RO, Day INM. Molecular Pathogenesis of Subarachnoid Haemorrhage. The International Journal of Biochemistry and Cell Biology 2003; 35:1341-60.

[82] Finlay HM, Whittaker P, Canham PB. Collagen Organization in the Branching Region of Human Brain Arteries. Stroke 1998; 29:1595.

[83] Stehbens W. Pathology and Pathogenesis of Intracranial Berry Aneurysms. Neurological Research 1990; 12:29.

[84] Kilic T, Sohrabifar M, Kurtkaya Ö, Yildirim Ö, Elmaci I, Günel M, Pamir MN. Expression of Structural Proteins and Angiogenic Factors in Normal Arterial and Unruptured and Ruptured Aneurysm Walls. Neurosurgery 2005; 57:997.

[85] Mitchell P, Gholkar A, Vindlacheruvu RR, David Mendelow A. Unruptured Intracranial Aneurysms: Benign Curiosity or Ticking Bomb? Lancet Neurology 2004; 3:85-92.

[86] Brisman JL, Song JK, Newell DW. Cerebral Aneurysms. New England Journal of Medicine 2006; 355:928-39.

[87] Broderick JP, Brott TG, Duldner JE, Tomsick T, Leach A. Initial and Recurrent Bleeding are the Major Causes of Death Following Subarachnoid Hemorrhage. Stroke 1994; 25:1342-7.

[88] Olafsson E, Hauser WA, Gudmundsson G. A Population based Study of Prognosis of Ruptured Cerebral Aneurysm: Mortality and Recurrence of Subarachnoid Hemorrhage. Neurology 1997; 48:1191-5.

[89] Ingall T, Asplund K, Mähönen M, Bonita R, Project ftWM. A Multinational Comparison of Subarachnoid Hemorrhage Epidemiology in the WHO MONICA Stroke Study. Stroke 2000; 31:1054-61.

[90] Bellebaum C, Schäfers L, Schoch B, Wanke I, Stolke D, Forsting M, Daum I. Clipping versus Coiling: Neuropsychological Follow up after Aneurysmal SubarachnoidHaemorrhage (SAH). Journal of Clinical and Experimental Neuropsychology 2004; 26:1081-92. 
[91] Brach RF. Coil or Clip? Current Trends in the Treatment of Intracranial Aneurysms. JAAPA : Official Journal of the American Academy of Physician Assistants 2009; 22:37-8, 40-2.

[92] Seibert B, Tummala RP, Chow R, Faridar A, Mousavi SA, Divani AA. Intracranial Aneurysms: Review of Current Treatment Options and Outcomes. Frontiers in Neurology 2011; 2:45.

[93] Germanwala AV, Zanation AM. Endoscopic Endonasal Approach for Clipping of Ruptured and Unruptured Paraclinoid Cerebral Aneurysms: Case Report. Neurosurgery 2011; 68:234-9.

[94] Zubillaga AF, Guglielmi G, Vi F, Duckwiler G. Endovascular Occlusion of Intracranial Aneurysms with Electrically Detachable Coils: Correlation of Aneurysm Neck Size and Treatment Results. American Journal of Neuroradiology 1994; 15:815-20.

[95] Horowitz M, Samson D, Purdy P. Does Electrothrombosis Occur Immediately after Embolization of an Aneurysm with Guglielmi Detachable Coils? American Journal of Neuroradiology 1997; 18:510.

[96] Kallmes DF, Borland MK, Cloft HJ, Altes TA, Dion JE, Jensen ME, Hankins G, Helm GA. In vitro Proliferation and Adhesion of Basic Fibroblast Growth Factor-Producing Fibroblasts on Platinum Coils. Radiology 1998; 206:237.

[97] Willinsky RA. Detachable Coils to Treat Intracranial Aneurysms. Canadian Medical Association Journal 1999; 161:1136.

[98] Choudhari KA, Flynn PA, McKinstry SC. Spontaneous Extrusion of Guglielmi Detachable Coils from Anterior Communicating Artery Aneurysm. Neurology India 2007; 55:148.

[99] Maitland D, Small IV W, Singhal P, Hwang W, Rodriguez J, Clubb F, Wilson T. Design and Realization of Biomedical Devices Based on Shape Memory Polymers. Materials Research Society Proceedings 2009; 1190:1190-NN06-01.

[100] Maitland DJ, Small IV W, Ortega JM, Buckley PR, Rodriguez J, Hartman J, Wilson TS. Prototype Laser-activated Shape Memory Polymer Foam Device for 
Embolic Treatment of Aneurysms. Journal of Biomedical Optics 2007; 12:030504.

[101] Tobushi H, Hara H, Yamada E, Hayashi S. Thermomechanical Properties in a Thin Film of Shape Memory Polymer of Polyurethane Series. Smart Materials and Structures 1996; 5:483-91.

[102] Tobushi H, Hashimoto T, Hayashi S, Yamada E. Thermomechanical Constitutive Modeling in Shape Memory Polymer of Polyurethane Series. Journal of Intelligent Material Systems and Structures 1997; 8:711.

[103] Tobushi H, Hashimoto T, Ito N, Hayashi S, Yamada E. Shape Fixity and Shape Recovery in a Film of Shape Memory Polymer of Polyurethane Series. Journal of Intelligent Material Systems and Structures 1998; 9:127-36.

[104] Tobushi H, Hayashi S, Hoshio K, Miwa N. Influence of Strain-holding Conditions on Shape Recovery and Secondary-shape Forming in PolyurethaneShape Memory Polymer. Smart Materials and Structures 2006; 15:1033.

[105] Tobushi H, Hayashi S, Kojima S. Mechanical Properties of Shape Memory Polymer of Polyurethane Series: Basic Characteristics of Stress-StrainTemperature Relationship. JSME International Journal Ser 1, Solid mechanics, Strength of Materials 1992; 35:296-302.

[106] Tobushi H, Okumura K, Hayashi S, Ito N. Thermomechanical Constitutive Model of Shape Memory Polymer. Mechanics of Materials 2001; 33:545-54.

[107] Gall K, Yakacki CM, Liu Y, Shandas R, Willett N, Anseth KS. Thermomechanics of the Shape Memory Effect in Polymers for Biomedical Applications. Journal of Biomedical Materials Research, Part A 2005; 73:339-48.

[108] Nguyen TD, Jerry Qi H, Castro F, Long KN. A Thermoviscoelastic Model for Amorphous Shape Memory Polymers: Incorporating Structural and Stress Relaxation. Journal of the Mechanics and Physics of Solids 2008; 56:2792-814.

[109] Cernošek Z, Holubová J, Cernošková E, Liška M. Enthalpic Relaxation and the Glass Transition. Journal of Optoelectronics and Advanced Materials 2002; 4:489-503. 
[110] Wilson T, Bearinger J, Herberg J, Marion III J, Wright W, Evans C, Maitland D. Shape memory polymers based on uniform aliphatic urethane networks. Journal of Applied Polymer Science 2007; 106:540-51.

[111] Gibson L, Ashby M. The Mechanics of Three-dimensional Cellular Materials. Proceedings of the Royal Society A: Mathematical, Physical and Engineering Science 1982; 382:43-59.

[112] Progelhof R, Throne J. Young's Modulus of Uniform Density Thermoplastic Foam. Polymer Engineering \& Science 1979; 19:493-9.

[113] Egli E. Design Properties of Structural Foam. Journal of Cellular Plastics 1972; $8: 245-9$.

[114] Cabanlit M, Maitland D, Wilson T, Simon S, Wun T, Gershwin ME, Van de Water J. Polyurethane Shape Memory Polymers Demonstrate Functional Biocompatibility In Vitro. Macromolecular Bioscience 2007; 7:48-55.

[115] Busby W, Cameron NR, Jahoda CAB. Emulsion-Derived Foams (PolyHIPEs) containing Poly ( $\varepsilon$-caprolactone) as Matrixes for Tissue Engineering. Biomacromolecules 2001; 2:154-64.

[116] Cooke MN, Fisher JP, Dean D, Rimnac C, Mikos AG. Use of Stereolithography to Manufacture Critical Sized 3D Biodegradable Scaffolds for Bone Ingrowth. Journal of Biomedical Materials Research, Part A 2003; 64:65-9.

[117] Cooper AI. Recent Developments in Materials Synthesis and Processing using Supercritical $\mathrm{CO}_{2}$. Advanced Materials 2001; 13:1111-4.

[118] Freyman T, Yannas I, Gibson L. Cellular Materials as Porous Scaffolds for Tissue Engineering. Progress in Materials Science 2001; 46:273-82.

[119] Hacker M, Ringhofer M, Appel B, Neubauer M, Vogel T, Young S, Mikos AG, Blunk T, Göpferich A, Schulz MB. Solid Lipid Templating of Macroporous Tissue Engineering Scaffolds. Biomaterials 2007; 28:3497-507.

[120] Harris LD, Kim BS, Mooney DJ. Open Pore Biodegradable Matrices Formed with Gas Foaming. Journal of Biomedical Materials Research 1998; 42:396-402. 
[121] Mikos AG, Temenoff JS. Formation of Highly Porous Biodegradable Scaffolds for Tissue Engineering. Electronic Journal of Biotechnology 2000; 3:23-4.

[122] Pham QP, Sharma U, Mikos AG. Electrospinning of Polymeric Nanofibers for Tissue Engineering Applications: A Review. Tissue Engineering 2006; 12:1197211.

[123] Shi X, Sitharaman B, Pham QP, Liang F, Wu K, Edward Billups W, Wilson LJ, Mikos AG. Fabrication of Porous Ultra-short Single-walled Carbon Nanotube Nanocomposite Scaffolds for Bone Tissue Engineering. Biomaterials 2007; 28:4078-90.

[124] Christenson EM, Soofi W, Holm JL, Cameron NR, Mikos AG. Biodegradable Fumarate-based polyHIPEs as Tissue Engineering Scaffolds. Biomacromolecules 2007; 8:3806-14.

[125] David D, Silverstein MS. Porous Polyurethanes Synthesized within High Internal Phase Emulsions. Journal of Polymer Science, Part A: Polymer Chemistry 2009; 47:5806-14.

[126] Jacobs L, Kemmere M, Keurentjes J, Mantelis CA, Meyer T. Temperature Induced Morphology Control in the Polymer Foaming Process. AIChE Journal 2007; 53:2651-8.

[127] Elwell MJ, Mortimer S, Ryan AJ. A Synchrotron SAXS Study of Structure Development Kinetics During the Reactive Processing of Flexible Polyurethane Foam. Macromolecules 1994; 27:5428-39.

[128] Elwell MJ, Ryan AJ, Gruenbauer HJM, Van Lieshout HC. In-situ Studies of Structure Development During the Reactive Processing of Model Flexible Polyurethane Foam Systems using FT-IR Spectroscopy, Synchrotron SAXS, and Rheology. Macromolecules 1996; 29:2960-8.

[129] Klempner D, Sendijarevic V, Aseeva RM. Handbook of Polymeric Foams and Foam Technology: Hanser Gardner Publications; 2004. 
[130] Shah D, Djabbarah N, Wasan D. A Correlation of Foam Stability with Surface Shear Viscosity and area per Molecule in Mixed Surfactant Systems. Colloid and Polymer Science 1978; 256:1002-8.

[131] Montminy MD, Tannenbaum AR, Macosko CW. The 3D Structure of Real Polymer Foams. Journal of Colloid and Interface Science 2004; 280:202-11.

[132] Jackovich D, O'toole B, Hawkins MC, Sapochak L. Temperature and Mold Size Effects on Physical and Mechanical Properties of a Polyurethane Foam. Journal of Cellular Plastics 2005; 41:153-68.

[133] Webster JG. Prevention of Pressure Sores - Engineering and Clinical Aspects. New York: Taylor and Francis group; 1991.

[134] Burdeniuc JJ, Andrew GD. Catalyst Composition for Water blown, Low density, Rigid Polyurethane Foam. US 2010/0152312 A1 Air Products and Chemicals, Inc. 2008.

[135] Haider K, Steppan D, Mcgregor M, Ingold K, Mautino V, Drobransky M. Polyol Compositions Useful for Preparing Dimensionally Stable, Low Density Water Blown Rigid Foams and the Processes Related Thereto. WO Patent WO/2004/000,907 Bayer Materials Science LLC 2003.

[136] Ning L, De-Ning W, Sheng-Kang Y. Hydrogen Bonding Properties of Segmented Polyether Poly(urethane urea) Copolymer. Macromolecules 1997; 30:4405-9.

[137] Yang B, Huang WM, Li C, Li L. Effects of Moisture on the Thermomechanical Properties of a Polyurethane Shape Memory Polymer. Polymer 2006; 47:134856.

[138] YIlgör E. Hydrogen Bonding and Polyurethane Morphology. I. Quantum Mechanical Calculations of Hydrogen Bond Energies and Vibrational Spectroscopy of Model Compounds. Polymer 2002; 43:6551-9.

[139] Zhang L, Jeon HK, Malsam J, Herrington R, Macosko CW. Substituting Soybean Oil-based Polyol into Polyurethane Flexible Foams. Polymer 2007; 48:6656-67. 
[140] Yakacki CM, Shandas R, Safranski D, Ortega AM, Sassaman K, Gall K. Strong, Tailored, Biocompatible Shape-Memory Polymer Networks. Advanced Functional Materials 2008; 18:2428-35.

[141] Ashby M, Medalist R. The Mechanical Properties of Cellular Solids. Metallurgical and Materials Transactions A 1983; 14:1755-69.

[142] Prima M, Di, Lesniewski M, Gall K, McDowell D, Sanderson T, Campbell D. Thermo-mechanical Behavior of Epoxy Shape Memory Polymer Foams. Smart Materials and Structures 2007; 16:2330-40.

[143] Mcgonigle EA, Cowie J, Arrighi V, Pethrick R. Enthalpy Relaxation and Free Volume Changes in Aged Styrene Copolymers Containing a Hydrogen Bonding Co-monomer. Journal of Materials Science 2005; 40:1869-81.

[144] Yang B, Huang W, Li C, Lee C, Li L. On the Effects of Moisture in a Polyurethane Shape Memory Polymer. Smart Materials and Structures 2004; 13:191.

[145] Huang W. Thermo-Moisture Responsive Polyurethane Shape Memory Polymer for Biomedical Devices. Open Medical Devices Journal 2010; 2:11-9.

[146] Huang WM, Yang B, Zhao Y, Ding Z. Thermo-moisture Responsive Polyurethane Shape-Memory Polymer and Composites: A Review. Journal of Materials Chemistry 2010; 20:3367.

[147] Jung YC, So HH, Cho JW. Water-Responsive Shape Memory Polyurethane Block Copolymer Modified with Polyhedral Oligomeric Silsesquioxane. Journal of Macromolecular Science: Physics 2006; 45:453-61.

[148] Fan K, Huang W, Wang C, Ding Z, Zhao Y, Purnawali H, Liew K, Zheng L. Water-responsive Shape Memory Hybrid: Design Concept and Demonstration. Express Polymer Letters 2011; 5:409.

[149] Wang CC, Huang WM, Ding Z, Zhao Y, Purnawali H. Cooling-/WaterResponsive Shape Memory Hybrids. Composites Science and Technology 2012; 72:1178-82. 
[150] Olson III RA, P.K. S. Method of Making Fine Pore Media. EP 1622695 Porvair PLC 2008.

[151] Geen H. Process for the Preparation of Reticulated Materials U.S. Pat. 3,175,030 Chemotronics Inc. MI 1965.

[152] Geen H, Rice W. Process for Bonding and/or Reticulation. U.S. Pat. 3,175,025 Chemotronics Inc. MI 1965.

[153] Haines R. Method for Leaching a Polyurethane Foam. U.S. Pat. 3,125,542 Owens-Corning Fiberglas Corp. DE 1964.

[154] Powers W, Volz R. Cellular Product and Method of Making the Same. U.S. Pat. 2,900,278 Scott Paper Company PA 1959.

[155] Stark N. Method for Making a Foamed Polyurethane. U.S. Pat. 2,961,710 Norman H. Stark 1960.

[156] Volz R. Reticulated Polyurethane Foams and the Process for their Production. U.S. Pat. 3,171,820 Scott Paper Company PA 1965.

[157] Mijovik JS, Koutsky JA. Etching of Polymeric Surfaces: A Review. Polymer Plastics Technology and Engineering 1977; 9:139-78.

[158] Van Krevelen DW, Nijenhuis KT. Properties of Polymers: Their Correlation with Chemical Structure, Their Numerical Estimation and Prediction from Additive Group Contributions. 4th ed. Amsterdam: Elsevier; 2009.

[159] Andrews EW, Gioux G, Onck P, Gibson LJ. Size Effects in Ductile Cellular Solids. Part II: Experimental Results. International Journal of Mechanical Sciences 2001; 43:701-13.

[160] Onck PR, Andrews EW, Gibson LJ. Size Effects in Ductile Cellular Solids. Part I: Modeling. International Journal of Mechanical Sciences 2001; 43:681-99.

[161] Welti D. Infrared Vapor Spectra: Group Frequency Correlations, Sample Handling and Examination of Gas Chromatographic Fractions. London: Heyden \& Sons; 1970.

[162] Socrates G. Infrared Characteristic Group Frequencies: Tables and Charts. Second ed: Wiley \& Sons, New York; 1994. 
[163] Hodge RM, Bastow TJ, Edward GH, Simon GP, Hill AJ. Free Volume and the Mechanism of Plasticization in Water-Swollen Poly(vinyl alcohol). Macromolecules 1996; 29:8137-43.

[164] Hartman J. Personal Communication. 2012.

[165] Singh H, Sharma TP, Jain AK. Reactivity of the Raw Materials and their Effects on the Structure and Properties of Rigid Polyurethane Foams. Journal of Applied Polymer Science 2007; 106:1014-23.

[166] Sipaut CS, Ahmad N, Adnan R, Rahman I, Mohamad Ibrahim M. Effects of Starting Material and Reaction Temperature on the Morphology and Physical Properties of Polyurethane Foams. Cellular Polymers 2010; 29:1-25.

[167] Li X, Cao H, Zhang Y. Properties of Water blown Rigid Polyurethane Foams with Different Functionality. Journal of Wuhan University of Technology-Mater Sci Ed 2008; 23:125-9.

[168] Lim H, Kim SH, Kim BK. Effects of the Hydroxyl Value of Polyol in Rigid Polyurethane Foams. Polymers for Advanced Technologies 2008; 19:1729-34.

[169] Kim S, Kim B, Lim H. Effect of Isocyanate Index on the Properties of Rigid Polyurethane Foams Blown by HFC 365mfc. Macromolecular Research 2008; 16:467-72.

[170] Mondal P, Khakhar DV. Regulation of Cell Structure in Water Blown Rigid Polyurethane Foam. Macromolecular Symposia 2004; 216:241-54.

[171] Kim SH, Lim H, Song JC, Kim BK. Effect of Blowing Agent Type in Rigid Polyurethane Foam. Journal of Macromolecular Science, Part A: Pure and Applied Chemistry 2008; 45:323-7.

[172] Kim SH, Lim H, Kim BK. Effects of Initiator Type in Rigid Polyurethane Foams. Polymer Engineering \& Science 2008; 48:1518-23.

[173] Mondal P, Khakhar D. Hydraulic Resistance of Rigid Polyurethane Foams III. Effect of Variation of the Concentration of Catalysts on Foam Structure and Properties. Journal of Applied Polymer Science 2004; 93:2838-43. 
[174] Mondal P, Khakhar D. Hydraulic Resistance of Rigid Polyurethane Foams. I. Effect of Different Surfactants on Foam Structure and Properties. Journal of Applied Polymer Science 2004; 93:2821-9.

[175] Mondal P, Khakhar D. Hydraulic Resistance of Rigid Polyurethane Foams. II. Effect of Variation of Surfactant, Water, and Nucleating agent Concentrations on Foam Structure and Properties. Journal of Applied Polymer Science 2004; 93:2830-7.

[176] Zhang XD, Macosko CW, Davis HT, Nikolov AD, Wasan DT. Role of Silicone Surfactant in Flexible Polyurethane Foam. Journal of Colloid and Interface Science 1999; 215:270-9.

[177] Harikrishnan G, Khakhar D. Effect of Monomer Temperature on Foaming and Properties of Flexible Polyurethane Foams. Journal of Applied Polymer Science 2007; 105:3439-43.

[178] Cole KC, Van Gheluwe P, Hébrard MJ, Leroux J. Flexible Polyurethane Foam. I. FTIR Analysis of Residual Isocyanate. Journal of Applied Polymer Science 2003; 34:395-407.

[179] Kim YH, Koczo K, Wasan DT. Dynamic Film and Interfacial Tensions in Emulsion and Foam Systems. Journal of Colloid and Interface Science 1997; 187:29-44.

[180] Scriven LE, Sternling CV. The Marangoni Effects. Nature 1960; 187:186-8.

[181] Kendrick TC, Kingston BM, Lloyd NC, Owen MJ. The Surface Chemistry of Polyurethane Foam Formation: I. Equilibrum Surface Tensions of Polysiloxanepolyether Block Copolymer Solutions. Journal of Colloid and Interface Science $1967 ; 24: 135-40$.

[182] Owen MJ, Kendrick TC. Surface Chemistry of Polyurethane Foam Formation: III. Effect of Gas Diffusion Between Bubbles and Surface Viscosity on Bubble Stability. Journal of Colloid and Interface Science 1968; 27:46-52. 
[183] Owen MJ, Kendrick TC, Kingston BM, Lloyd NC. The Surface Chemistry of Polyurethane Foam Formation: II. The Role of Surface Elasticity. Journal of Colloid and Interface Science 1967; 24:141-50.

[184] Hild F, Biering D. Hydrophobic Insulation Material. US Pat. Appl. No. US 2009/0061200 A1 TriStar Plastics Corporation, Shrewsbury, MA 2007.

[185] Bittencourt JA. Fundamentals of Plasma Physics. Third ed. New York: SpringerVerlag; 2004.

[186] Guarino V, Ambrosio L. The Synergic Effect of Polylactide Fiber and Calcium Phosphate Particle Reinforcement in Poly \&-caprolactone-based Composite Scaffolds. Acta Biomaterialia 2008; 4:1778-87.

[187] Vaquette C, Kahn C, Frochot C, Nouvel C, Six JL, De Isla N, Luo LH, CooperWhite J, Rahouadj R, Wang X. Aligned Poly(L-lactic-co-e-caprolactone) Electrospun Microfibers and Knitted Structure: A Novel Composite Scaffold for Ligament Tissue Engineering. Journal of Biomedical Materials Research Part A 2010; 94:1270-82.

[188] Chen H, Huang J, Yu J, Liu S, Gu P. Electrospun Chitosan-graft-poly ( $\varepsilon$ caprolactone)/poly ( $\varepsilon$-caprolactone) Cationic Nanofibrous Mats as Potential Scaffolds for Skin Tissue Engineering. International Journal of Biological Macromolecules 2011; 48:13-9.

[189] Li W-J, Cooper Jr JA, Mauck RL, Tuan RS. Fabrication and Characterization of Six Electrospun Poly( $\alpha$-hydroxy ester)-based Fibrous Scaffolds for Tissue Engineering Applications. Acta Biomaterialia 2006; 2:377-85.

[190] Liu JJ, Wang CY, Wang JG, Ruan HJ, Fan CY. Peripheral Nerve Regeneration using Composite Poly(lactic acid-caprolactone)/Nerve Growth Factor Conduits Prepared by Coaxial Electrospinning. Journal of Biomedical Materials Research Part A 2011; 96:13-20.

[191] Nisbet DR, Rodda AE, Horne MK, Forsythe JS, Finkelstein DI. Neurite Infiltration and Cellular Response to Electrospun Polycaprolactone Scaffolds Implanted into the Brain. Biomaterials 2009; 30:4573-80. 
[192] Heydarkhan-Hagvall S, Schenke-Layland K, Dhanasopon AP, Rofail F, Smith H, Wu BM, Shemin R, Beygui RE, MacLellan WR. Three-Dimensional Electrospun ECM-based Hybrid Scaffolds for Cardiovascular Tissue Engineering. Biomaterials 2008; 29:2907-14.

[193] Ulery BD, Nair LS, Laurencin CT. Biomedical Applications of Biodegradable Polymers. Journal of Polymer Science Part B Polymer Physics 2011; 49:832-64.

[194] Behl M, Zotzmann J, Schroeter M, Lendlein A. Biodegradable Shape Memory Polymers. In: Lendlein A, Sisson A, editors. Handbook of Biodegradable Polymers: Synthesis, Characterization and Applications. First ed: Wiley-VCH Verlag GmbH \& Co.; 2011.

[195] Alteheld A, Feng Y, Kelch S, Lendlein A. Biodegradable, Amorphous Copolyester Urethane Networks Having Shape Memory Properties. Angewandte Chemie International Edition 2005; 44:1188-92.

[196] Choi N-Y, Lendlein A. Degradable shape-memory polymer networks from oligo[(1-lactide)-ran-glycolide]dimethacrylates. Soft Matter 2007; 3:901-9.

[197] Choi N-Y, Kelch S, Lendlein A. Synthesis, Shape-Memory Functionality and Hydrolytical Degradation Studies on Polymer Networks from Poly (rac-lactide)b-poly (propylene oxide)-b-poly (rac-lactide) Dimethacrylates. Advanced Engineering Materials 2006; 8:439-45.

[198] Göpferich A. Mechanisms of Polymer Degradation and Erosion. Biomaterials 1996; 17:103-14.

[199] Oberth AE, Bruenner RS. Effect of Hydrogen Bonding on the Kinetics of the Urethane Reaction. Journal of Physical Chemistry 1968; 72:845-55.

[200] Elwell MJ, Ryan AJ, Grünbauer HJM, Van Lieshout HC. In-Situ Studies of Structure Development during the Reactive Processing of Model Flexible Polyurethane Foam Systems Using FT-IR Spectroscopy, Synchrotron SAXS, and Rheology. Macromolecules 1996; 29:2960-8. 


\section{APPENDIX A}

\section{PHYSICO-CHEMICAL PROCESSES DURING FOAM BLOWING}

This appendix discusses the physical and chemical processes that underlie the fabrication of foams via the gas blowing method of foam synthesis. As discussed in this dissertation, synthesis of the foams (proposed here) is performed in three steps:

\section{A. NCO Premix}

First an isocyanate (NCO) premix is prepared by addition of $35-45 \%$ hydroxyl equivalents to the isocyanate component. As this premix is allowed to cure, end-capping of $35-40 \%$ of isocyanates with hydroxyl groups (HPED, TEA or PCL-t) occurs, leading to the formation of relatively longer polymer chains. By changing the $\%$ hydroxyl equivalents added in this premix, the viscosity of the solution can be effectively controlled in the range of $\sim 2$ to 60 Pa.s.

\section{B. OH Premix}

In the next step, a hydroxyl $(\mathrm{OH})$ premix is prepared. This premix contains the balance stoichiometric amounts of hydroxyl monomers and chemical blowing agent (water), along with the surfactants and catalysts. It is prepared just prior to the foaming process.

\section{Foaming}

In the third step, the $\mathrm{NCO}$ and $\mathrm{OH}$ premixes are mixed together in predetermined 
ratios, with the physical blowing agent (Enovate; HFC 245-fa), and placed in an oven at $90{ }^{\circ} \mathrm{C}$. The blowing begins at this point; starting from the mixing of reactive components to the end foam product, the process can be divided into four primary phases based on the extent of reaction and development of the porous foam morphology (Figure 50):

\section{Phase I}

On mixing the $\mathrm{OH}$ and $\mathrm{NCO}$ groups together, we initially have a homogeneous liquid mixture comprising isocyanates end capped with hydroxyl groups (from the prepolymer), unreacted isocyanate and hydroxyl monomers, $\mathrm{OH}$ polyol (it has been shown that hydroxyl groups associate with each other via hydrogen bonding and this process assists in the polyurethane reaction by weakening the $\mathrm{O}-\mathrm{H}$ bond of the alcohols) [199], foaming additives (surfactants, catalysts, and blowing agents), and some urea hard segments from the moisture that may get in the mixture from atmospheric moisture.

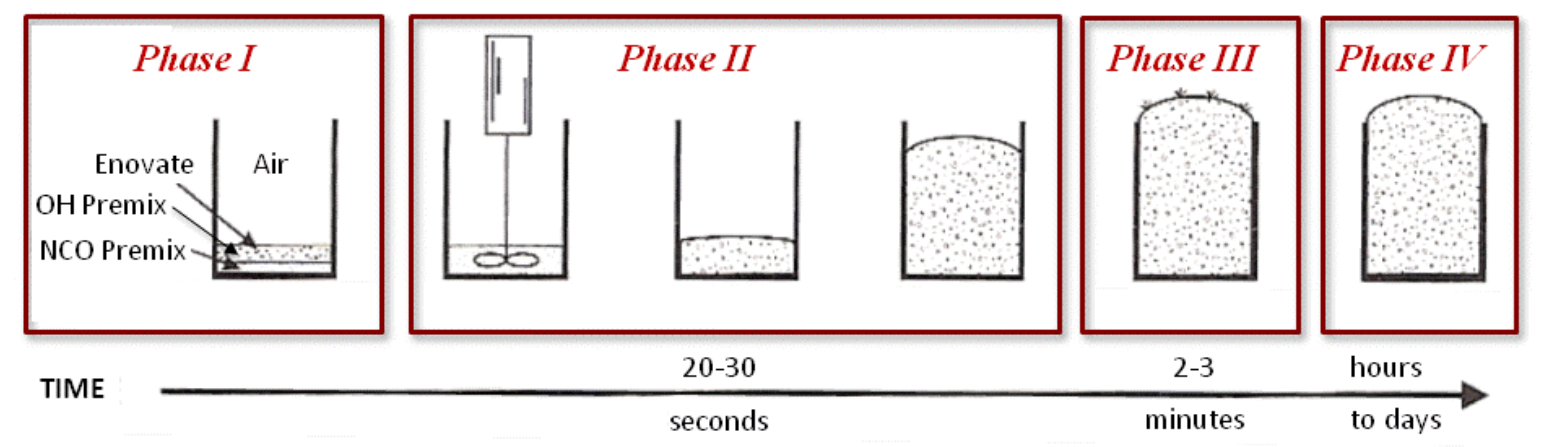

Figure 50: The four main phases of generation of a foam via the gas-blowing method: Phase I- Mixing of reactive components, Phase II- Foam rise, Phase III- Gelation/Vitrification, and Phase IV- Further cure. 


\section{Phase II}

The reaction continues via formation of urea and urethane linkages as we mix the components together. As the degree of polymerization increases, traditional segmented polyurethanes undergo a thermodynamic instability at a certain degree of polymerization

of the hard segment $\left(N_{h}\right)$. This thermodynamic incompatibility is explained by the FloryHuggins theory, and at this point the value of $\chi N_{h}$, where $\chi$ is the Flory-Huggins interaction parameter, reaches a critical value. In traditional polyurethanes, this has been shown to happen at approximately $40-50 \%$ of the isocyanate conversion [200]. The thermodynamic instability drives the microphase separation of the urea segments from the urethane linkages, forming discrete balls or interdispersed areas of urea hard segment in the polymer. This phase separation is shown to occur by spinodal decomposition [200], and takes place uniformly over the entire material. The covalently crosslinked amorphous materials discussed in this dissertation, however, do not undergo this microphase separation; they form a monolith amorphous crosslinked structure.

Another significant process occurring simultaneously with the chemical reaction is the foam expansion and development of the porous foam morphology. Basic source of foam expansion is the generation of gas, either $\mathrm{CO}_{2}$ from the isocyanate-water reaction, or from the physical blowing agent that gets activated in the increased temperature; either from the heat of the oven $\left(90{ }^{\circ} \mathrm{C}\right)$, or due to the exothermic reaction (the foaming is shown to be a quasi-adiabatic process and the temperatures of the interior of foam quickly rise to around $80{ }^{\circ} \mathrm{C}$ ). The generation of porous morphology of the foams is based on the formation of polyhedral polymer structures around the air bubbles. As the 
air bubble nucleates and grows in size, these polyhedral structures generate defined areas of polymer meniscus, and polymer lamella (Figure 51). The meniscus has a larger reservoir of polymer, which limits its polymer-air interface relative to the lamella. As a result, a gradient in the surface tension is developed at the polymer-air interface stretching from the lamella to the meniscus (Figure 52). This gradient causes the drainage of polymer from the lamella to the meniscus, leading to the thinning of the future "cell membrane" of the foam. Viscosity of the polymer, as well as the rate of increase in viscosity with the polymerization reaction, play an important part in the determination of the rate and extent of polymer drainage, and hence in the stability and final morphology of the foams. For instance, a very high rate of drainage of low viscosity solutions may destabilize the foam and lead to its collapse, while a very slow rate of drainage of high viscosity solutions may lead to a primarily closed cell structure with thick cell membranes. Surfactants in the polymer solution hence play a critical role by changing its surface tension, stabilizing the polymer-air interface, and thereby modulating the rate of drainage of the polymer from the lamella. Similarly, catalysts are very important for ensuring that the polymerization reaction occurs at an adequate pace to allow sufficient time for polymer drainage, while simultaneously generating the mechanical strength necessary for supporting the growing structure.

With continued drainage of the polymer to the meniscus, cell opening may eventually be achieved [179]. However, this requires a very fine balance between the polymer drainage and foam stability. Hence, complete cell opening of all the cells is typically not seen in the as-made blown foams [129]. 


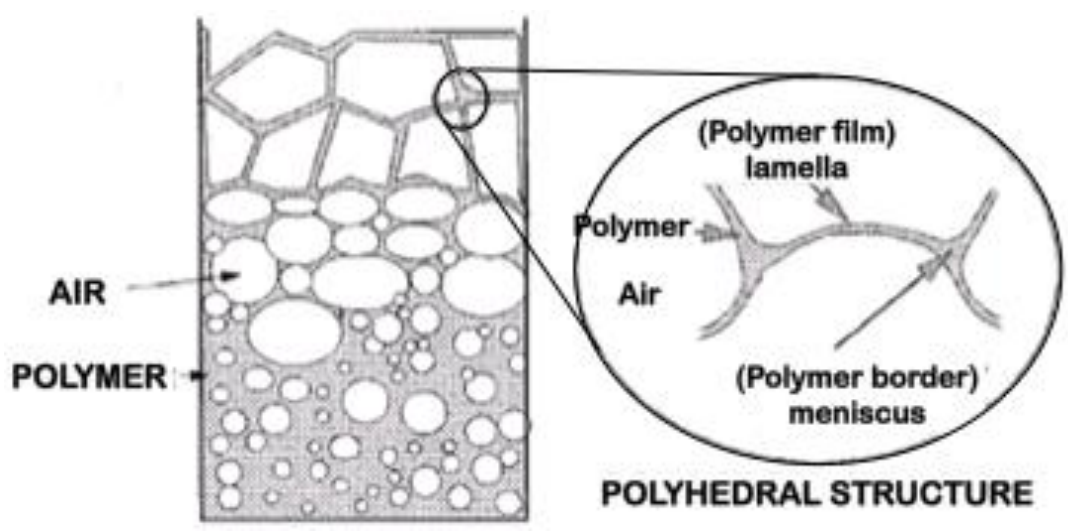

Figure 51: Formation of polyhedral structure of foam cells around the gas bubbles with defined regions of lamella and meniscus (Adapted with permission from [179]).

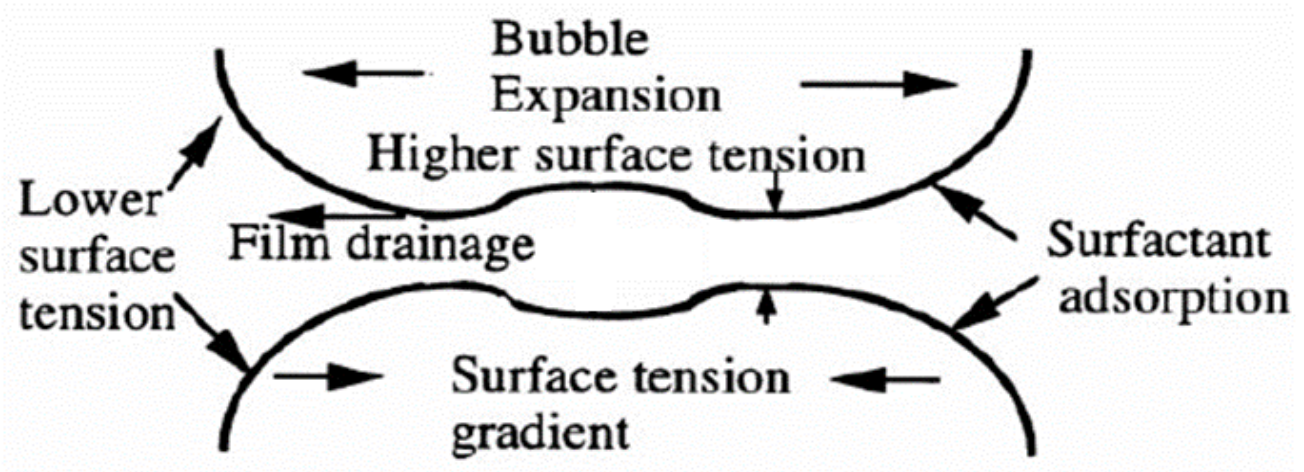

Figure 52: A surface tension gradient occurs from the lamella to the meniscus, which leads to the drainage of polymer into the meniscus, thereby thinning the lamella (or the future cell membranes). Adapted with permission from [176].

\section{Phase III}

At approximately $70 \%$ isocyanate conversion (for traditional polyurethane foams), vitrification/physical gelation of the polymer takes place. This is defined as the Berghman's point, and vitrification of the polymer at this stage freezes in the foam 
morphology. In other words, this transition means that the phase has attained a composition with $T_{g}$ equal to the temperature of the reacting system, and the foam morphology and phase separation is arrested at this composition by the glass transition (Berghman's point). It is also known as the physical gel point or the end of rise time. The modulus increases by almost a factor of 25 for typical polyurethane foams at this point.

\section{Phase IV}

After vitrification, the reaction still continues, albeit in a medium with increasing viscosity and stiffness. Any $\mathrm{CO}_{2}$ that is released at this stage gets entrapped in the closed cell (is not able to rupture the cell membrane, but can reversibly expand the elastomeric membrane) [200]. 


\section{APPENDIX B}

\section{FOAM PREPARATION FOR MECHANICAL TESTING}

This appendix describes the method that was used for preparing the foam samples for the DMTA tests in ARES rheometer (TA Instruments Inc.). DMTA was performed in the torsional mode using rectangular samples of foams, $\sim 45 \mathrm{~mm}$ long, 12 $\mathrm{mm}$ wide, and $6 \mathrm{~mm}$ thick. To maintain the low density foam structure while clamping, and to avoid the damage of the samples from the metal clamps, their ends were embedded in a low $T_{g}$ neat polyurethane material (Urethane RTV System- Shore A-30, TAP Plastics Inc.).

a)

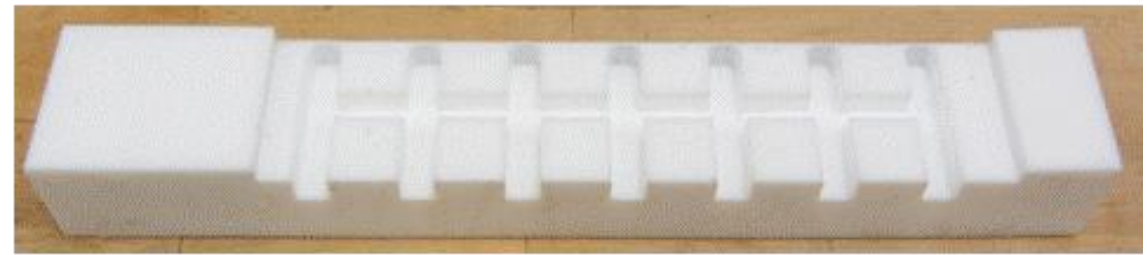

b)
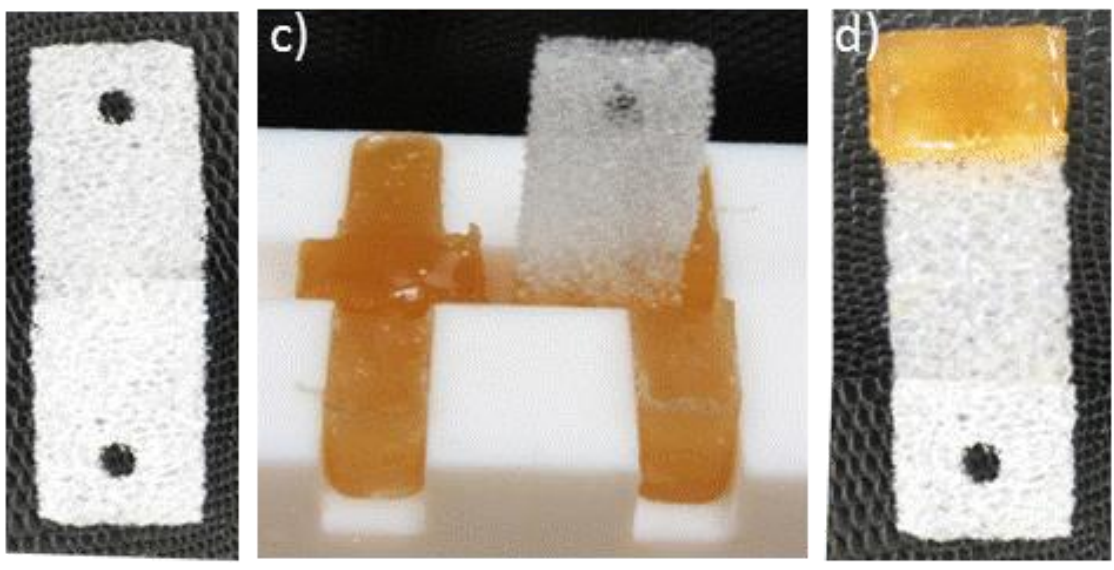

Figure 53: Preparation of foam sample for DMTA tests. 
A custom made Teflon mold was used for this purpose (Figure 53 a). A hole was punched though the foam in the area to be submerged in the polymer resin, to allow better permeation and adhesion of the neat polymer with the foam (Figure $53 \mathbf{b}$ ). Thereafter, the foam sample was placed in the mold well and the polyurethane solution was poured in. This assembly was allowed to cure for $\sim 2-3$ hours (Figure 53 c). After cure, the sample was released from the mold (Figure 53 d), and the process was repeated on the other side. The neat polyurethane (Urethane RTV System- Shore A-30, TAP Plastics Inc.) used here is in its rubbery state at room temperature $\left(20-30{ }^{\circ} \mathrm{C}\right)$. This offers an additional advantage of being able to trim the external dimensions of the cast, as per the requirements, after embedding the foam. 


\section{APPENDIX C}

\section{DETERMINATION OF SOLUBILITY PARAMETER}

Hoy's solubility parameters were calculated for determining the chemical compatibility of the different polymer compositions with water (Chapter III). Hoy's solubility parameter is based on an addition group contribution theory that uses the following equations for amorphous polymeric systems:

\section{A. Additive Molar Functions}

The net values of molar functions are calculated as a sum of contribution of individual units " $i$ " where $N_{i}$ is the number of the " $i$ "th" unit in a given molecular structure. Here, $F_{t}$ is the molar attractive force, and $F_{p}$ is the polar component of this molar attractive force. $\Delta_{T}^{(P)}$ and $V$ represent the non-ideality correction factor and molar volume respectively.

$$
\begin{aligned}
& F_{t}=\Sigma\left(N_{i} \times F_{t, i}\right) \\
& \text { (Eqn C. 1) } \\
& F_{p}=\Sigma\left(N_{i} \times F_{p, i}\right) \\
& \text { (Eqn C. 2) } \\
& V=\Sigma\left(N_{i} \times V_{i}\right) \\
& \text { (Eqn C. 3) } \\
& \Delta_{T}^{(P)}=\Sigma\left(N_{i} \times \Delta_{T_{i}^{i}}^{(P)}\right)
\end{aligned}
$$

\section{B. Auxiliary Equations}


Auxiliary equations define the molecular aggregation number for amorphous polymers $\alpha(P)$, number of repeating units per effective polymer chain segment $n$, and a base value $B$.

$$
\begin{gathered}
\alpha(P)=777 \times \Delta_{T}^{(P)} / V \\
n=0.5 / \Delta_{T}^{(P)} \\
\mathrm{B}=277
\end{gathered}
$$

(Eqn C. 7)

\section{Expressions for $\delta$ and $\delta$ Components}

The solubility parameter $\delta_{t}$, and the contribution to this value from the constituent polar forces $\left(\delta_{p}\right)$, hydrogen bonding forces $\left(\delta_{h}\right)$, and dispersive forces $\left(\delta_{d}\right)$ are calculated as follows:

$$
\begin{gathered}
\delta_{t}=\left(F_{i}+\frac{B}{n}\right) / V \\
\delta_{p}=\delta_{t}\left(\frac{1}{\alpha^{(P)}} \frac{F_{P}}{F_{t}+\frac{B}{n}}\right)^{1 / 2} \\
\delta_{h}=\delta_{t}\left[\frac{\alpha^{(P y}-1}{\alpha^{(P)}}\right]^{1 / 2} \\
\delta_{d}=\left(\delta_{t}^{2}-\delta_{p}^{2}-\delta_{h}^{2}\right)^{1 / 2}
\end{gathered}
$$

Values of the $\delta$ components are first calculated based on the molecular groups present in the polymer structure of the constituent structural units. $\delta$ of the polymer is then calculated for the entire composition based on the volume fraction of individual structural units. The net $\delta$ is derived at as the square root of the sum of the squares of $\delta_{d}$, $\delta_{p}$ and $\delta_{h}$ values. 
This process is demonstrated in Table $\mathbf{1 6}$ for the 100TM composition (Chapter III). The 4 structural units (Figure 23) were identified in the polymer, and the $\delta$ values for each were calculated from individual group contributions [158].

Table 15: Typical values of solubility parameters for some common polymers [158].

\begin{tabular}{|c|c|c|c|c|c|}
\hline Polymer & From & to & Polymer & From & to \\
\hline Polyethylene & 15.8 & 17.1 & Poly (butyl methacrylate) & 17.8 & 18.4 \\
\hline Polypropylene & 16.8 & 18.8 & Poly (isobutyl methacrylate) & 16.8 & 21.5 \\
\hline Polyisobutylene & 16.0 & 16.6 & Poly (tert-butyl methacrylate) & 17.0 & - \\
\hline Polystyrene & 17.4 & 19.0 & $\begin{array}{c}\text { Poly (ethoxyethyl } \\
\text { methacrylate) }\end{array}$ & 18.4 & 20.3 \\
\hline Poly (vinyl chloride) & 19.2 & 22.1 & Polyacrylonitrile & 25.6 & 31.5 \\
\hline Poly (vinyl bromide) & 19.4 & - & Polymethacrylonitrile & 21.9 & - \\
\hline Poly (vinylidene chloride) & 20.3 & 25 & Poly ( $\alpha$-cyanomethyl acrylate) & 28.7 & 29.7 \\
\hline Poly (tetrafluoroethylene) & 12.7 & - & Polybutadiene & 16.6 & 17.6 \\
\hline $\begin{array}{c}\text { Poly } \\
\text { (chlorotrifluoroethylene) }\end{array}$ & 14.7 & 16.2 & Polyisoprene & 16.2 & 20.5 \\
\hline Poly (vinyl alcohol) & 25.8 & 29.1 & Polychloroprene & 16.8 & 18.9 \\
\hline Poly (vinyl acetate) & 19.1 & 22.6 & Polyformaldehyde & 20.9 & 22.5 \\
\hline Poly (vinyl propionate) & 18.0 & - & Poly (tetramethylene oxide) & 17.0 & 17.5 \\
\hline Poly (methyl acrylate) & 19.9 & 21.3 & Poly (propylene oxide) & 15.4 & 20.3 \\
\hline Poly (ethyl acrylate) & 18.8 & 19.2 & Polyepichlorohydrin & 19.2 & - \\
\hline Poly (propyl acrylate) & 18.5 & - & Poly (ethylene sulphide) & 18.4 & 19.2 \\
\hline Poly (butyl acrylate) & 18.0 & 18.6 & Poly (styrene sulphide) & 19.0 & - \\
\hline Poly (isobutyl acrylate) & 17.8 & 22.5 & Poly (ethylene terephthalate) & 19.9 & 21.9 \\
\hline $\begin{array}{c}\text { Poly (heptafluorobutyl } \\
\text { acrylate) }\end{array}$ & 13.7 & - & Poly (8-aminocaprylic acid) & 26.0 & - \\
\hline Poly (methyl methacrylate) & 18.6 & 26.2 & $\begin{array}{c}\text { Poly (hexamethylene } \\
\text { adipamide) }\end{array}$ & 27.8 & - \\
\hline Poly (ethyl methacrylate) & 18.2 & 18.7 & & & \\
\hline
\end{tabular}


Table 16: Calculation of Hoy's solubility parameters for the 100TM composition (Chapter III).

\begin{tabular}{|c|c|c|c|c|c|c|c|c|c|c|c|c|c|c|c|c|}
\hline & $n_{i}$ & $F t_{i}$ & $F p_{i}$ & $V_{i}$ & $\Delta T i$ & $\Delta T i^{(P)}$ & $F_{t}$ & $F p$ & $V$ & $\Delta T^{(P)}$ & $\alpha_{(p)}$ & $n$ & $\delta_{t}$ & $\delta_{p}$ & $\delta_{h}$ & $\delta_{d}$ \\
\hline \multicolumn{17}{|c|}{ HPED unit } \\
\hline$+\mathrm{CH}_{2}+$ & 18 & 269 & 0 & 15.55 & 0.02 & 0.02 & 4842 & 0 & 279.9 & 0.36 & & & & & & \\
\hline$\stackrel{\stackrel{\mathrm{O}}{\mathrm{II}}}{+\mathrm{NH}-\mathrm{C}-\mathrm{O}+}$ & 4 & 1265 & 890 & 34.8 & 0.08 & 0.09 & 5060 & 3560 & 139.2 & 0.38 & & & & & & \\
\hline$\left.+\mathrm{CH}_{3}\right)$ & 10 & 303.5 & 0 & 21.55 & 0.02 & 0.02 & 3035 & 0 & 215.5 & 0.22 & & & & & & \\
\hline $\begin{array}{c}\mathrm{I} \\
-\mathrm{C} \\
\mathrm{I}\end{array}$ & 4 & 176 & 0 & 9.56 & 0.01 & 0.01 & 704 & 0 & 38.24 & 0.05 & & & & & & \\
\hline${ }_{1} \mathrm{~N}-$ & 2 & 125 & 125 & 12.6 & 0.01 & 0.01 & 250 & 250 & 25.2 & 0.02 & & & & & & \\
\hline & & & & & & & 13891 & 3810 & 698.04 & 1.03 & 1.14 & 0.49 & 20.71 & 9.95 & 7.31 & 16.63 \\
\hline \multicolumn{17}{|c|}{ TEA unit } \\
\hline$+\mathrm{CH}_{2}+$ & 15 & 269 & 0 & 15.55 & 0.02 & 0.02 & 4035 & 0 & 233.25 & 0.3 & & & & & & \\
\hline$\stackrel{\stackrel{\mathrm{O}}{I I}}{+\mathrm{NH}-\mathrm{C}-\mathrm{O}+}$ & 3 & 1265 & 890 & 34.8 & 0.08 & 0.09 & 3795 & 2670 & 104.4 & 0.28 & & & & & & \\
\hline $\mathrm{N}^{\mathrm{N}}-$ & 1 & 125 & 125 & 12.6 & 0.01 & 0.01 & 125 & 125 & 12.6 & 0.01 & & & & & & \\
\hline$\left.+\mathrm{CH}_{3}\right)$ & 4.5 & 303.5 & 0 & 21.55 & 0.02 & 0.02 & 1365.75 & 0 & 96.98 & 0.10 & & & & & & \\
\hline & & & & & & & 7955 & 2795 & 350.25 & 0.59 & 1.31 & 0.85 & 23.65 & 12.00 & 11.52 & 16.81 \\
\hline \multicolumn{17}{|c|}{ Water unit } \\
\hline$+\mathrm{CH}_{2}+$ & 6 & 269 & 0 & 15.55 & 0.02 & 0.02 & 1614 & 0 & 93.3 & 0.12 & & & & & & \\
\hline$\stackrel{\mathrm{O}}{\text { II }}$ & 1 & 1499 & 1263 & 39.3 & 0.09 & 0.10 & 1499 & 1263 & 39.3 & 0.10 & & & & & & \\
\hline
\end{tabular}


Table 16 continued.

\begin{tabular}{|c|c|c|c|c|c|c|c|c|c|c|c|c|c|c|c|c|}
\hline & $n_{i}$ & $F t_{i}$ & $F p_{i}$ & $V_{i}$ & $\Delta T i$ & $\Delta T i^{(P)}$ & $F_{t}$ & $F p$ & $V$ & $\Delta T^{(P)}$ & $\alpha_{(p)}$ & $n$ & $\delta_{t}$ & $\delta_{p}$ & $\delta_{h}$ & $\delta_{d}$ \\
\hline \multirow[t]{2}{*}{$\left.+\mathrm{CH}_{3}\right)$} & 3 & 303.5 & 0 & 21.55 & 0.02 & 0.02 & 910.5 & 0 & 64.65 & 0.07 & & & & & & \\
\hline & & & & & & & 4023.5 & 1263 & 197.25 & 0.29 & 1.13 & 1.75 & 21.20 & 10.97 & 7.16 & 16.67 \\
\hline \multicolumn{17}{|c|}{ Remaining unit } \\
\hline$+\mathrm{CH}_{2}+$ & 3 & 269 & 0 & 15.55 & 0.02 & 0.02 & 807 & 0 & 46.65 & 0.06 & & & & & & \\
\hline$\left.+\mathrm{NH}_{2}\right)$ & 1 & 464 & 464 & 17 & 0.031 & 0.035 & 464 & 464 & 17 & 0.04 & & & & & & \\
\hline \multirow[t]{2}{*}{$\left.+\mathrm{CH}_{3}\right)$} & 1.5 & 303.5 & 0 & 21.55 & 0.023 & 0.022 & 455.25 & 0 & 32.325 & 0.03 & & & & & & \\
\hline & & & & & & & 1726.25 & 464 & 95.98 & 0.13 & 1.04 & 3.91 & 18.73 & 9.35 & 3.50 & 15.84 \\
\hline
\end{tabular}

Table 17: Solubility parameters for each structural unit.

\begin{tabular}{|c|c|c|c|c|}
\hline & $\boldsymbol{\delta}_{\boldsymbol{t}}$ & $\boldsymbol{\delta}_{\boldsymbol{p}}$ & $\boldsymbol{\delta}_{\boldsymbol{h}}$ & $\boldsymbol{\delta}_{\boldsymbol{d}}$ \\
\hline HPED & 10.21 & 4.90 & 3.60 & 8.20 \\
\hline TEA & 3.92 & 1.99 & 1.91 & 2.78 \\
\hline Water & 6.61 & 3.42 & 2.23 & 5.20 \\
\hline Remaining & 0.56 & 0.28 & 0.10 & 0.47 \\
\hline Net values & 21.29 & 10.59 & 7.84 & 16.65 \\
\hline
\end{tabular}

Table 18: Volume fraction of components using rule of mixtures

\begin{tabular}{|c|c|c|c|}
\hline Component & Volume & $\begin{array}{c}\text { Mole } \\
\text { fraction }\end{array}$ & Volume fraction \\
\hline HPED & 698.04 & 0.23 & 0.49 \\
\hline TEA & 350.25 & 0.15 & 0.17 \\
\hline Water & 197.25 & 0.52 & 0.31 \\
\hline Remaining & 95.98 & 0.10 & 0.03 \\
\hline
\end{tabular}




\section{APPENDIX D}

\section{BLOWING AGENT NOMENCLATURE}

The foam additive industries offer a wide variety of physical blowing agents for use in the gas blowing process. Physical blowing agents used in fabrication of foams are typically volatile liquids with low boiling point comprising halogenated hydrocarbons (Chlorofluorocarbons (CFCs), Hydrofluorocarbons (HFCs), Hydrochlorofluorocarbons (HCFC) and Perfluorocarbons (PFC)), low boiling alcohols, ethers, aromatic hydrocarbons, or solid adsorbents saturated with gases or low boiling liquids. While a detailed description of the benefits and drawbacks of these different categories of blowing agents may be found elsewhere [129], the objective of this appendix is to discuss the otherwise ambiguous nomenclature (e.g. HCFC-141b, HFC 245 fa) used by the halogenated hydrocarbons to codify their molecular structure.

\section{A. Rules for Decoding the Name of CFC, HCFC, HFC, PFC etc. for Molecular Structure}

\section{Decoding the Initial Letters- Determining Constituents}

First " $\mathrm{C}$ " of the name represents chlorine and the second or only " $\mathrm{C}$ " represents carbon. It is noteworthy that the $\mathrm{C}-\mathrm{Cl}$ bonds of CFCs and HCFCs can undergo photolytic scission in the presence of high energy UV radiations, producing radicals that can be ozone depleting. HFCs and PFCs do not have "Cl" (one or only $\mathrm{C}$ represents carbon), 
and hence are non-ozone depleting. Further, "F" and "PF" denote "Fluoro" and "Perfluoro" structures respectively.

2. Decoding the Number-Determining Bond Structure (using HCFC-141b for example)

2.1 Add 90 to the number $141+90=231$.

2.2. The digits of the calculated sum represent the number of carbon, hydrogen and fluorine atoms in the molecular structure respectively; $(\# \mathrm{C}=2, \# \mathrm{H}=3, \# \mathrm{~F}=1)$.

2.3 For calculating $\mathrm{Cl}$, count the number of $\mathrm{C}$ bonds required to make a saturated molecule $(2 \mathrm{C}+2=6$ bonds; with $3 \mathrm{H}$ 's and $1 \mathrm{~F}$, we will need $2 \mathrm{Cl}$ 's for a saturated molecule).

3. Decoding the Final Letter-Determining Arrangement of Bonds

\subsection{Structures with 2 Carbons}

3.1.1 Get the total number of atomic weights of atoms bonded to each carbon atom.

3.1.2 The arrangement distributing the atomic weights most evenly is not designated with a letter.

3.1.3 Arrangement that is the next most even arrangement is designated by an " $a$ ".

3.1.4 Next most even arrangement is designated by "b".

\subsection{Structures with 3 Carbons}


3.2.1 Fist letter indicates the atoms in the middle carbon ( $2 \mathrm{C} 1=$ "a"; $\mathrm{Cl}, \mathrm{F}=$ "b"; 2 $\mathrm{F}=$ "“c"; $\mathrm{H}, \mathrm{Cl}=$ "“d"; $\mathrm{H}, \mathrm{F}=$ "ee'; $2 \mathrm{H}=$ "“f”).

3.2.2 Second letter indicates symmetry in outside carbons (most symmetric="a", next symmetric="b", and so on).

\section{B. Example - Decoding Enovate}

Enovate, used in the synthesis of foams discussed in this dissertation, is a nonchlorinated blowing agent depicted as HFC-245 fa. Using the above rules, its name can be decoded to derive the molecule structure as follows:

\section{Determining the Constituents}

One and only $\mathrm{C}$ denotes presence of carbon, and $\mathrm{H}$ and $\mathrm{F}$ denote a saturated molecule with bonds consumed by hydrogen and fluorine respectively.

\section{Determining the Bond Structure}

$$
\text { 245+90=335 }(\# \mathrm{C}=3, \# \mathrm{H}=3, \# \mathrm{~F}=5) \text {. }
$$

\section{Determining the Bond Arrangement (3 carbon)}

"fa" indicates that the middle carbon has 2 hydrogens, and the remaining atoms are arranged in the most symmetric manner ("a"), leading to the following configuration of atoms: 
<smiles>FCCCC(F)(F)F</smiles>

Figure 54: Structure of Enovate as determined from its name HFC-245fa

\section{B. Halons}

Halons directly show the $\mathrm{C}, \mathrm{F}, \mathrm{Cl}, \mathrm{Br}$ numbers (no addition of 90 is required). For example, Halon 1211 represents 2 fluorine atoms, and 1 each of carbon, chlorine and bromine $(\# \mathrm{C}=1, \# \mathrm{~F}=2, \# \mathrm{Cl}=1, \# \mathrm{Br}=1)$. 